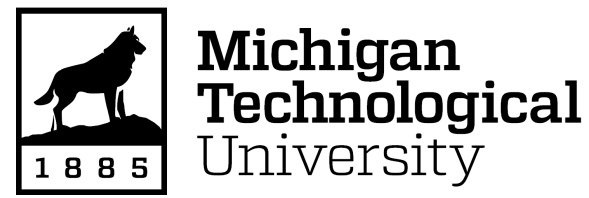

Michigan Technological University Digital Commons @ Michigan Tech

Dissertations, Master's Theses and Master's Reports

2019

\title{
CHARACTERIZING FRESHWATER PHYTOPLANKTON DYNAMICS WITH ELECTRO-OPTICAL REMOTE SENSING
}

Michael Sayers

Michigan Technological University, mjsayers@mtu.edu

Copyright 2019 Michael Sayers

\section{Recommended Citation}

Sayers, Michael, "CHARACTERIZING FRESHWATER PHYTOPLANKTON DYNAMICS WITH ELECTROOPTICAL REMOTE SENSING", Open Access Dissertation, Michigan Technological University, 2019.

https://doi.org/10.37099/mtu.dc.etdr/910

Follow this and additional works at: https://digitalcommons.mtu.edu/etdr

Part of the Biogeochemistry Commons, Environmental Monitoring Commons, Fresh Water Studies Commons, Oceanography Commons, and the Water Resource Management Commons 


\title{
CHARACTERIZING FRESHWATER PHYTOPLANKTON DYNAMICS WITH ELECTRO-OPTICAL REMOTE SENSING
}

By

Michael J. Sayers

\begin{abstract}
A DISSERTATION
Submitted in partial fulfillment of the requirements for the degree of DOCTOR OF PHILOSOPHY

In Geology
\end{abstract}

MICHIGAN TECHNOLOGICAL UNIVERSITY

2019

(C) 2019 Michael J. Sayers 
This dissertation has been approved in partial fulfillment of the requirements for the Degree of DOCTOR OF PHILOSOPHY in Geology.

Department of Geological and Mining Engineering and Sciences

\author{
Dissertation Co-Advisor: Robert Shuchman \\ Dissertation Co-Advisor: Thomas Oommen \\ Committee Member: Gary Fahnenstiel \\ Committee Member: Guy Meadows
}

Department Chair: John S. Gierke 


\section{Table of Contents}

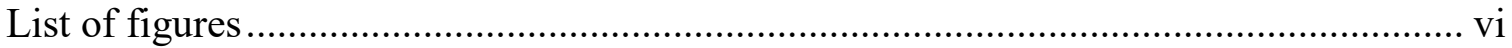

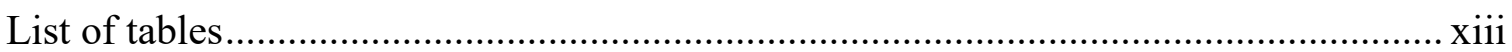

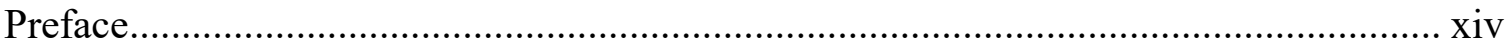

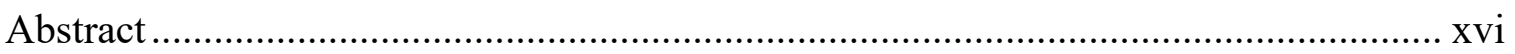

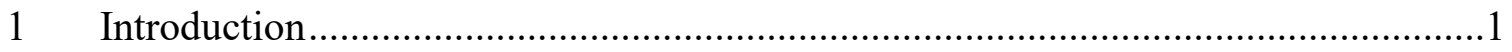

2 A New Method to Generate a Global Distribution Map of Lake Chlorophyll ...........9

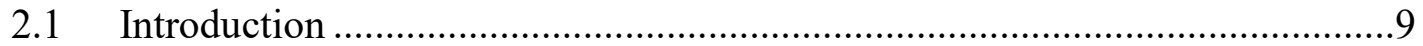

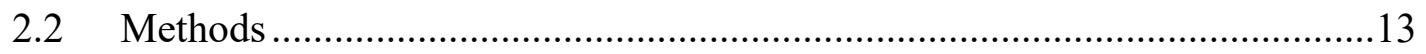

2.2.1 Global-scale estimation of inland lake chl concentrations ...............13

2.2.1.1 Limitations of NASA standard products...........................13

2.2.1.2 Sensor selection ............................................................14

2.2.1.3 MTRI method and generation of global chlorophyll snapshot 17

2.2.2 Validation with in situ data and ancillary environmental

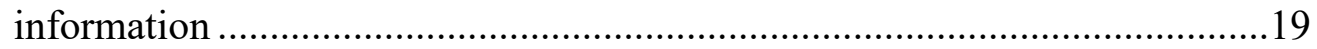

2.2.2.1 Global in situ chl dataset...........................................19

2.2.3 Global Lakes and Wetlands Database............................................22

2.2.3.1 Ancillary environmental data.........................................22

2.2.4 Matching in situ and satellite-derived chl data ...............................22

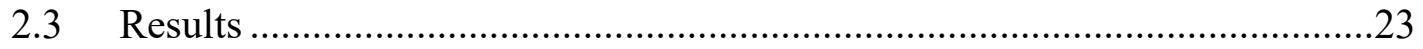

2.3.1 MERIS satellite-derived vs. in situ chl estimates .............................23

2.3.2 Comparison of the new MTRI method and standard L2 product .....26

2.3.3 Comparison across ecoregions..........................................................

2.3.3.1 Freshwater Ecoregions of the World ...............................31

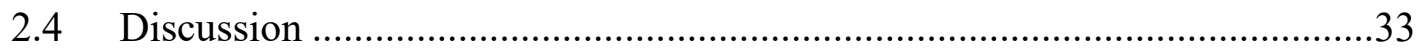

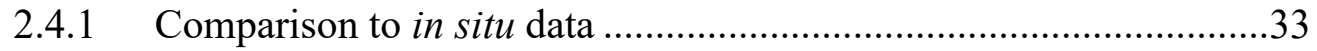

2.4.2 Comparison of chl distributions for MERIS L3 product and new

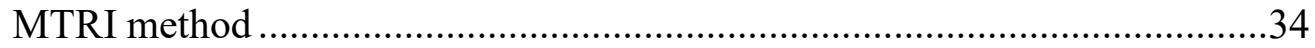

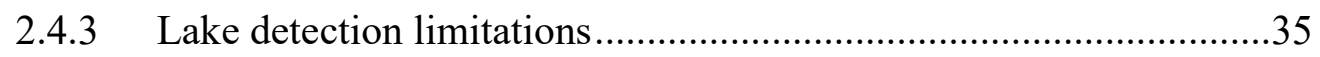

2.4.4 Future potential of Landsat 8 ......................................................... 


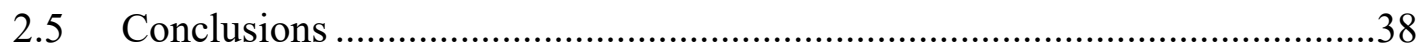

2.6 Acknowledgements ................................................................................

3 Global Carbon Fixation from Freshwater Lakes .....................................................40

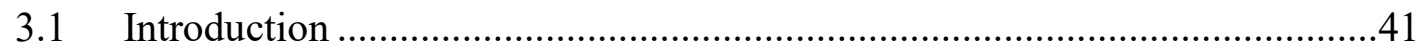

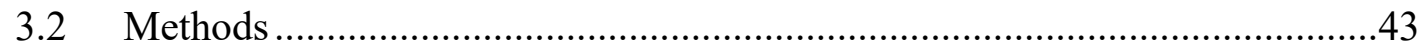

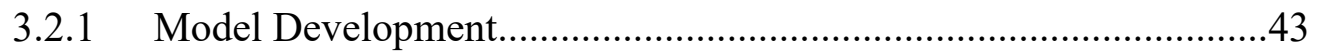

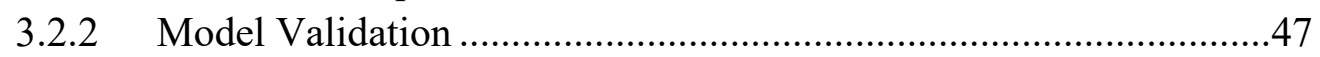

3.2.3 Remote Sensing Model Application ................................................48

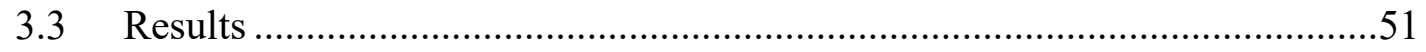

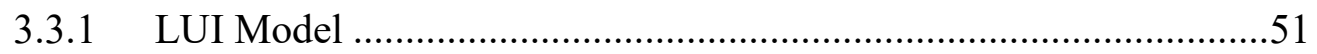

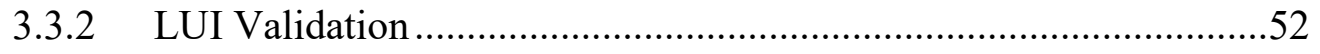

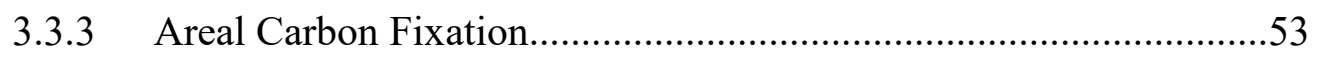

3.3.4 Total Carbon Fixation ....................................................................5

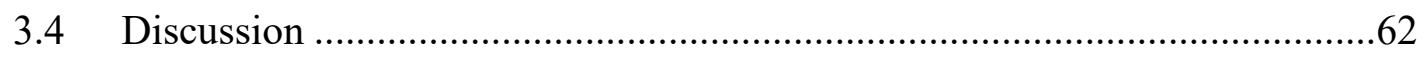

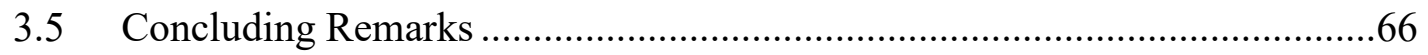

4 Cyanobacteria blooms in three eutrophic basins of the Great Lakes: A comparative analysis using satellite remote sensing ............................................................................67

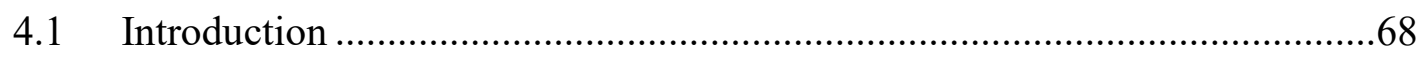

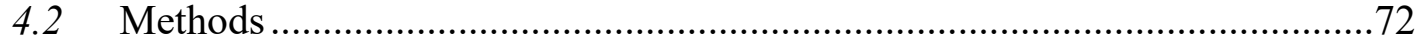

4.2.1 Field and environmental data.......................................................

4.2.2 CyanoHAB mapping and detection ................................................73

4.2.3 Validation of cyanoHAB mapping method ......................................78

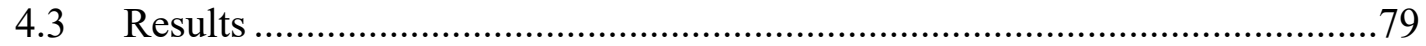

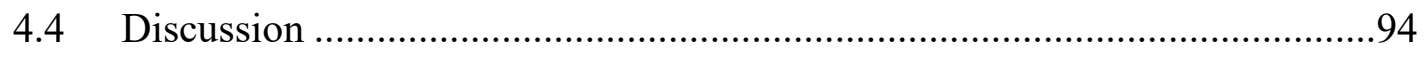

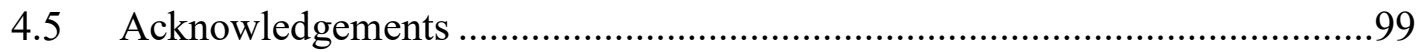

5 Satellite Monitoring of Harmful Algal Blooms in the Western Basin of Lake Erie: a

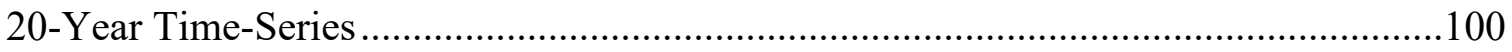

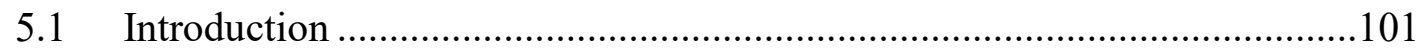

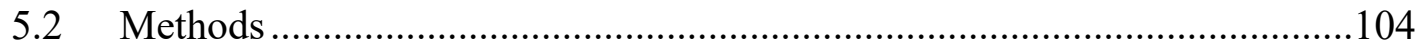

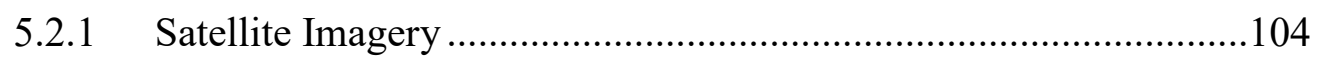

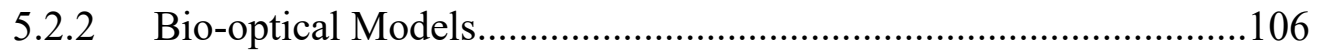




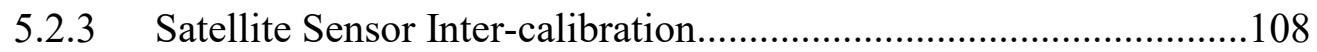

5.2.4 SeaWiFS Product Verification.....................................................109

5.2.5 SeaWiFS and MODIS Product Inter-comparison.............................110

5.2.6 Time-series Analysis....................................................................111

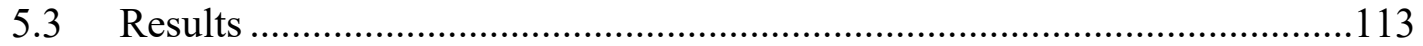

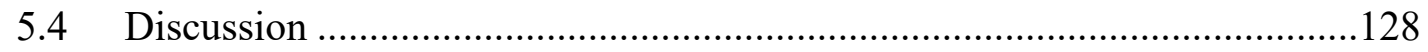

5.5 Conclusions and Recommendations..........................................................133

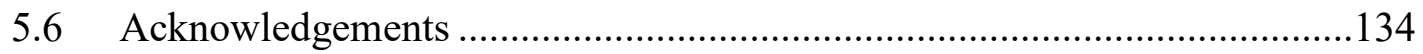

6 Spatial and temporal variability of inherent and apparent optical properties in western Lake Erie: Implications for water quality remote sensing...................................135

6.1 Introduction and Background .................................................................136

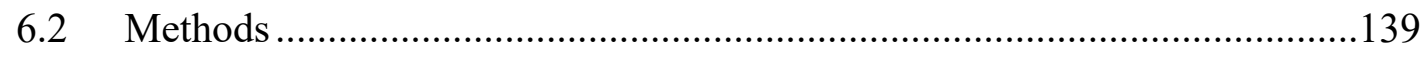

6.2.1 Study Area ……..................................................................139

6.2.2 Biogeochemical / Laboratory Data .................................................140

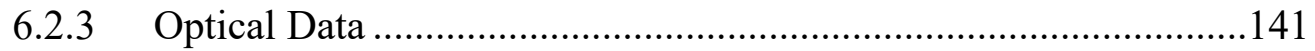

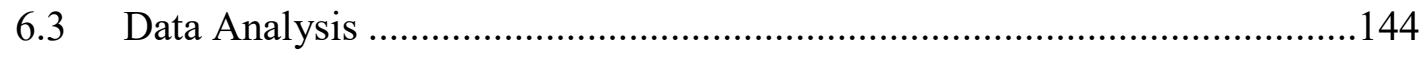

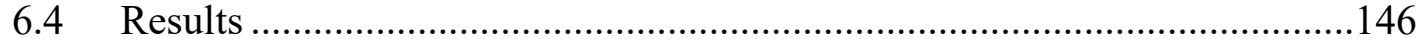

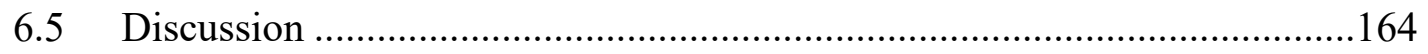

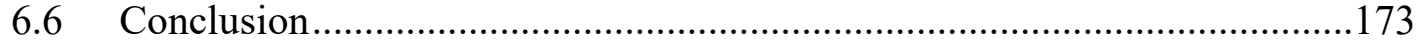

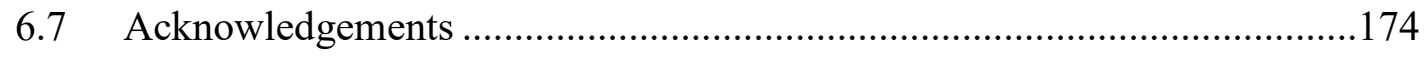

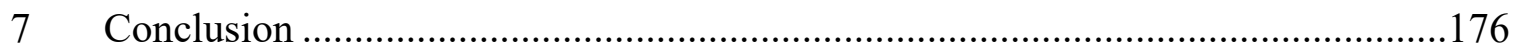

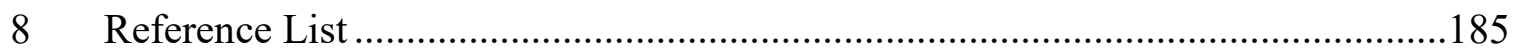




\section{List of figures}

Figure 2.1. Example of the area of surface water retrieved by the MODIS L3 4-km product (represented in green) and the additional areas gained by using the MODIS 1-km composite (in yellow) and MERIS FRS imagery (in red) for an area in the Canadian Shield $\left(63.0^{\circ} \mathrm{N}, 99.0^{\circ} \mathrm{W}\right)$. MERIS retrieves the most information (green + yellow + red areas). The inset map at top left indicates the location of the example area. 15

Figure 2.2. Log-log plot of lake size vs. global lake frequency, annotated with the minimum resolvable lake sizes for MODIS 4-km and 1-km composites and MERIS 300-m data. Column values derived from Verpoorter, Kutser, and Tranvik 2014 16

Figure 2.3. Steps involved in the MTRI method for processing MERIS OC4 chl data at the global scale.

Figure 2.4. Locations of the 37 lakes for which in situ chlorophyll- $a$ data from one or more dates were compared to MERIS OC4 values for the same lake and time period.

Figure 2.5. Comparison of chl values measured in situ vs. derived from MERIS ocean colour satellite imagery on a log-log scale ranging from 0 to 100 for 37 unique lakes distributed across the globe ( $\mathrm{n}=185$ observations). Point size corresponds to $\log$ lake surface area $\left(\mathrm{km}^{2}\right)$. Solid black line is identity line; $\mathrm{RMSE}=9.39, \mathrm{R}^{2}=$ 0.72 .

Figure 2.6. Comparison of chlorophyll estimates from MERIS L2 data for an area of the Canadian Shield (top panel). Of the $\sim 700$ water bodies present within the red box, 65 were resolved in an L2 image using the NASA ODPS land mask and 634 were resolved in the same image processed without the mask. For illustration, the area outlined in green in the top panel is shown in greater Description of 2011 snapshot data and global patterns

Figure 2.7. World distribution of the density of lakes from the GLWD shapefile that are visible in the MERIS 2011 global snapshot composite when the land mask was not used for data processing. Lake densities are represented as the number of lakes whose centre points fall within $50 \mathrm{~km}$ of the pixel (an area of approx. 7850 $\mathrm{km}^{2}$ ). Note that lakes are heavily concentrated around $40-70^{\circ} \mathrm{N}$. 
Figure 2.8. Histogram of mean lake-wide chl concentrations for the $\sim 80,000$ inland lakes from Global Lakes and Wetlands Database Levels 1 and 2 that could be retrieved with the MERIS 2011 global growing-season snapshot for world freshwater lakes. The mean chlorophyll-a value was $19.2 \pm 19.2 \mathrm{mg} \mathrm{m}-3$ and the median was 13.3 $\mathrm{mg} \mathrm{m}$

Figure 2.9. Density plot of mean lake-wide values of chl vs. the latitude of the lake's centre point for the approx. 80,000 lakes resolvable in the MERIS global growing season snapshot for the year 2011 .

Figure 2.10. Mean MERIS-derived chl value $(a)$ and coefficient of variation $(\mathrm{CoV})(b)$ for each of Abell et al.'s (2008) Freshwater Ecoregions of the World. Light grey ecoregions include no retrieved lakes $(a)$ or fewer than three retrieved lakes $(b)$. The high CoV within many ecoregions indicates that lakes that are not retrieved by MERIS cannot be easily estimated based on their neighbors.

Figure 2.11. Distributions of lake-wide chl values in the MTRI and NASA OceanColor L3 products for global summer 2011 (August for Northern Hemisphere lakes, February for Southern Hemisphere). Both histograms are normalized by the total number of lakes resolved in the respective products. .35

Figure 2.12. Percentage of the lakes included in GLWD Levels 1 and 2 for which chl values could be retrieved from the 2011 growing season global snapshot, separated into several lake surface area size classes.

Figure 3.1. Relationship of light utilization index $(\psi)$ and latitude for global freshwater lakes during the growing season. Because approximately $80 \%$ of our observations were from latitudes 35-52 degrees, we bin averagedthe latitude data (3 degrees). The decreasing linear relationship between latitude and LUI was significant ( $\mathrm{p}$-value $=0.001, \mathrm{R}^{2}=0.57$ ).

Figure 3.2. Mean growing season areal carbon fixation $\left(\mathrm{mg} \mathrm{C} / \mathrm{m}^{2} / \mathrm{d}\right)$ in freshwater lakes aggregated by freshwater ecoregions of the world (Abell et al. 2008).

Figure 3.3. Areal carbon fixation $\left(\mathrm{mg} \mathrm{C} / \mathrm{m}^{2} / \mathrm{d}\right)$ aggregated by freshwater ecoregions of the world vs. latitude for northern $\left(\mathrm{y}=-13.479 \mathrm{x}+1279.3, \mathrm{R}^{2}=0.45, \mathrm{p}<0.001, \mathrm{n}=\right.$ 217 and southern hemispheres $\left(\mathrm{y}=-14.781 \mathrm{x}+1227.1, \mathrm{R}^{2}=0.26, \mathrm{p}=0.046, \mathrm{n}=\right.$ 16).

Figure 3.4. Mean continental growing season areal carbon fixation $\left(\mathrm{mg} \mathrm{C} / \mathrm{m}^{2} / \mathrm{d}\right)$. .55 
Figure 3.5. Log-log plots of individual lake area and areal carbon fixation for all 80000 lakes grouped by hemisphere (northern - left panel, southern - right panel)........57

Figure 3.8. Log-log plots of total lake surface area and total carbon fixation for lakes aggregated by freshwater ecoregions of the world. Data points are colored by groups (Red - N 0-30 degrees, Yellow - N 30-60 degrees, Green - N >60 degrees, Blue $-\mathrm{S}$ 0-30 degrees, Purple $-\mathrm{S}$ 30-60 degrees).

Figure 4.1. Map of areas analyzed for cyanoHAB occurrences in the Great Lakes. Hatched areas indicate the basins included in analysis. The dominant river (nutrient source) for each basin is also labeled on the map. .70

Figure 4.2. Surface chlorophyll $a$ concentration vs. phycocyanin concentration measured in WBLE during the 2011 July-October bloom period. .76

Figure 4.3. $\mathrm{MCH}$ derived mean annual cyanoHAB extent for $(a)$ WBLE, $(b) \mathrm{SB}$, and $(c)$ GB from 2002-2013

Figure 4.4. SSI derived mean annual surface scum extent for (a) WBLE, (b) SB, and (c) GB from 2002-2013.

Figure 4.5. CyanoHAB occurrence maps for WBLE from 2002-2013. Colored areas indicate the number of days cyanoHABs were present while areas in black experienced no cyanoHAB presence.

Figure 4.6. CyanoHAB occurrence heat maps for SB from 2002-2013. Colored areas indicate the number of days cyanoHABs were present areas in black experienced no cyanoHAB presence.

Figure 4.7. CyanoHAB occurrence heat maps for GB from 2002-2013. Colored areas indicate the number of days cyanoHABs were present while areas in black experienced no cyanoHAB presence.

Figure 4.8. Mean annual air temperature for WBLE, GB, and SB from 2002-2013 „......87

Figure 4.9. Mean annual precipitation for WBLE, GB, and SB from 2002-2013. .88

Figure 4.10. (a) Mean annual river discharge, $Q$, for WBLE (Maumee River), GB (Fox River), and SB (Saginaw River). (b) Mean spring (March-June) river discharge, $Q$, for WBLE (Maumee River), GB (Fox River), and SB (Saginaw River). Note the $y$-axis scale ranges are different. 
Figure 4.11. (a) SSI mean annual extent vs. spring discharge for WBLE $\left(y=0.0008 x^{2}\right.$ $\left.0.2844 x+29.226, R^{2}=0.75, p<0.05\right)$. (b) $\mathrm{MCH}$ mean annual extent vs. spring discharge for WBLE with 2013 removed as a statistical outlier $(y=0.84 x+155$, $\left.R^{2}=0.50, p<0.05\right)$. (c) SSI mean annual extent vs. spring discharge for SB $(p>$ $0.05)$. (d) MCH mean annual extent vs. spring discharge for SB $(p>0.05)$. (e) SSI mean annual extent vs. spring discharge for GB $(p>0.05)$. $(f) \mathrm{MCH}$ mean annual extent vs. spring discharge for GB $(p>0.05)$.

Figure 4.12. The number of wave-induced sediment resuspension event occurrence in the cyanoHAB bloom period (July-September) for WBLE from 2002-2013. 92

Figure 5.1: The western Lake Erie study area is defined by the hatched area. The Maumee River is also shown as bold black line entering the basin. 106

Figure 5.2. Comparison of SSI-based scum classification results from MODIS (left panel), the initial SeaWiFS result (center panel), and the corrected SeaWiFS result (right panel) for June 22, 2003. Black cells represent positive surface scum observations.

Figure 5.3. Cloud free images (black dots) used to generate combined 20 year time series data set. SeaWiFS images were used from 1998-2001 and MODIS from 20022017. Also shown (black X) are the 20 degree onset and offset dates established from satellite surface water temperature observations.

Figure 5.4. Comparison of mean and maximum annual cyanoHAB areas based on the $\mathrm{MCH}$ (a, top panel) and surface scum areas based on the SSI (b, bottom panel) in the Western Basin of Lake Erie for SeaWiFS (1998-2007) and MODIS Aqua (2002-2017).

Figure 5.5. (a, left panel) Annual mean cyanoHAB extent $\left(\mathrm{km}^{2}\right)$ from the $\mathrm{MCH}$ with a linear best fit line (slope $=25.28, \mathrm{R}^{2}=0.34, \mathrm{p}=0.007$ ) and $95 \%$ confidence interval shaded in grey and (b, right panel) annual maximum cyanoHAB extent with a linear best fit line ( slope $=57.7, \mathrm{R}^{2}=0.54, \mathrm{p}<0.001$ ) and $95 \%$ confidence interval shaded in grey. Points from 1999-2001 come from the SeaWiFS sensor; points from 2002-2017 come from the MODIS Aqua sensor

Figure 5.6. (a, left panel) Annual mean scum extent $\left(\mathrm{km}^{2}\right)$ from the SSI with a linear best fit line (slope $=2.42, \mathrm{R}^{2}=0.39, \mathrm{p}=0.003$ ) and $95 \%$ confidence interval shaded in grey and ( $b$, right panel) annual maximum cyanoHAB extent with a linear best fit line (slope $=11.29, \mathrm{R}^{2}=0.39, \mathrm{p}=0.003$ ) and 95\% confidence interval shaded in 
grey. Points from 1999-2001 come from the SeaWiFS sensor; points from 20022017 come from the MODIS Aqua sensor. Note the linear fits in the figure are not intended for scum area prediction. 120

Figure 5.7. a, left panel, Mean total cyanoHAB extent (floating and nonfloating) over the 20 year data record in Western Basin Lake Erie plotted with standard deviation errors. b, right panel, Mean 8-day seasonal pattern of total cyanoHAB extent in Western Basin Lake Erie, plotted with standard deviation error bars.

Figure 5.8. Frequency occurrence annual heat maps of cyanoHAB presence for the 19982017 period. Areas of more frequent occurrence are shown in warmer colors while areas of no-occurrence are shown in black. Data from 1999-2001 come from the SeaWiFS sensor; data from 2002-2017 come from the MODIS Aqua sensor

Figure 5.9. Cumulative frequency occurrence heat maps of total cyanoHABs (left) and surface scum (right) for the 1998-2017 period. Warmer colors indicate more frequent occurrence.

Figure 5.10. Relationships between Maumee River weighted spring discharge and (a) $\mathrm{MCH}$ mean annual extent linear fit $\left(\mathrm{y}=1.53 \mathrm{x}+64.14, \mathrm{p}=0.02, \mathrm{R}^{2}=0.27\right)$, (b) $\mathrm{MCH}$ mean annual extent exponential fit $\left(\mathrm{y}=165.92 * 10^{\wedge}(0.022 \mathrm{x}), \mathrm{p}=0.002, \mathrm{R}^{2}\right.$ $=0.39)$, (c) SSI mean annual extent cubic fit $\left(\mathrm{y}=0.002 \mathrm{x}^{3}+0.09 \mathrm{x}^{2}-3.11 \mathrm{x}+\right.$ 25.92, $\mathrm{p}<0.001, \mathrm{R}^{2}=0.71$ ), and (d) SSI mean annual extent exponential fit ( $\mathrm{y}=$ $\left.1.84^{*} 10^{\wedge}(0.047 x), p=0.002, R^{2}=0.45\right)$. Plotted lines represent the best fits lines for each relationship. Open circles are the extents derived from SeaWiFS data, closed circles are from MODIS data reported in Sayers et al. (2016), and open triangles are from MODIS data reported in this study.

Figure 6.1. Station names and locations from 2015 and 2016 weekly data collections. Stations sampled regularly (weekly) are shown as black dots. Basin bathymetry is shown as varying shades of gray with darker colors indicating deeper water depth

Figure 6.2. Temporal patterns of chlorophyll-a (Top panel), phycocyanin (middle panel), and fixed suspended solids (bottom panel) in western Lake Erie expressed as boxplots where the black horizontal line is the median, the bottom and top of the box are the first and third quartiles respectively, and the horizontal gray lines below and above the box are the minimum and maximum values respectively. 
Monthly median values for 2015 (left half) and 2016 (right half) are shown in all three panels.

Figure 6.3. Monthly mean beam attenuation, absorption, and scattering coefficients for 2015 and 2016. Absolute values are shown in the left panels while mean normalized spectra are shown in the right panels. Lines are colored by month where solid lines represent 2015 data and dashed lines 2016 data. Note absolute value spectra (left panels) are on different y-axis scales.

Figure 6.4. Station mean (2015 and 2016 combined) beam attenuation, absorption, and scattering coefficients. Absolute values are shown in the left panels while mean normalized spectra are shown in the right panels. Lines are colored by station location. Note absolute value spectra (left panels) are on different y-axis scales.

Figure 6.5. Spatial and temporal patterns of $a_{p h} * 665$ in western Lake Erie expressed as boxplots where the black horizontal line is the median, the bottom and top of the box are the first and third quartiles respectively, and the horizontal gray lines below and above the box are the minimum and maximum values respectively. Monthly median values for 2015 (left half) and 2016 (right half) are shown in the top panel. Station median values for the combined 2015-2016 dataset are shown in the middle panel. Annual station median values are shown in the bottom panel.

Figure 6.6. Comparison between particulate absorption phycocyanin line height $\left(\mathrm{PCLH}_{\mathrm{ap}}\right)(\mathrm{x}$-axis) and laboratory extracted phycocyanin concentration (Lab $\mathrm{PC})\left(\mathrm{y}\right.$-axis). Note the $\log _{10}$ scales.

Figure 6.7. Spatial and temporal patterns of the particulate absorption phycocyanin line height (PCLH) in western Lake Erie expressed as boxplots where the black horizontal line is the median, the bottom and top of the box are the first and third quartiles respectively, and the horizontal gray lines below and above the box are the minimum and maximum values respectively. Monthly median values for 2015 (left half) and 2016 (right half) are shown in the top panel. Station median values for the combined 2015-2016 dataset are shown in the middle panel. Annual station median values are shown in the bottom panel. 156

Figure 6.8. Spatial and temporal patterns of the CDOM spectral slope, $\mathrm{S}_{\mathrm{CDOM}}$, in western Lake Erie expressed as boxplots where the black horizontal line is the median, the bottom and top of the box are the first and third quartiles respectively, and the 
horizontal gray lines below and above the box are the minimum and maximum values respectively. Monthly median values for 2015 (left half) and 2016 (right half) are shown in the top panel. Station median values for the combined 20152016 dataset are shown in the middle panel. Annual station median values are shown in the bottom panel.

Figure 6.9. Spatial and temporal patterns of the particulate beam attenuation spectral slope, $\gamma$, in western Lake Erie expressed as boxplots where the black horizontal line is the median, the bottom and top of the box are the first and third quartiles respectively, and the horizontal gray lines below and above the box are the minimum and maximum values respectively. Monthly median values for 2015 (left half) and 2016 (right half) are shown in the top panel. Station median values for the combined 2015-2016 dataset are shown in the middle panel. Annual station median values are shown in the bottom panel.

Figure 6.10. Temporal patterns in remote sensing reflectance $\left(\mathrm{R}_{\mathrm{rs}}, \mathrm{sr}^{-1}\right)$ in western Lake Erie for 2015 (top panels) and 2016 (bottom panels). The monthly mean spectra are displayed as red lines. Variability in monthly spectra are also shown in the gray-black regions around the mean spectra where each color represents a percentage of measured $\mathrm{R}_{\mathrm{rs}}$ values, following the method described by Hochberg et al. (2003) (Light gray $=2.5-97.5 \%$, medium gray $=12.5-87.5 \%$, dark gray $=$ $25-75 \%$ and black $=37.5-62.5 \%$ ). Note, there were no $R_{\mathrm{rs}}$ observations for May and October 2016.

Figure 6.11. Study period mean remote sensing reflectance $\left(\mathrm{R}_{\mathrm{rs}}, \mathrm{sr}^{-1}\right)$ for stations WE4 (top panels) and WE6 (bottom panels). Absolute $\mathrm{R}_{\mathrm{rs}}$ (left panels) and normalized $\mathrm{R}_{\mathrm{rs}}$ (right panels) are shown for both stations. The study period mean spectra are displayed as red lines. Variability in study period spectra are also shown in the gray-black regions around the mean spectra where each color represents a percentage of measured $\mathrm{R}_{\mathrm{rs}}$ values, following the method described by Hochberg et al. $(2003)($ Light gray $=2.5-97.5 \%$, medium gray $=12.5-87.5 \%$, dark gray $=$ $25-75 \%$ and black $=37.5-62.5 \%)$.

Figure 6.12. Comparison between particulate absorption phycocyanin line height $\left(\mathrm{PCLH}_{\mathrm{ap}}\right)(\mathrm{y}$-axis) and remote sensing reflectance phycocyanin line height (PCLH $\left.{ }_{\text {Rrs }}\right)(\mathrm{x}$-axis). Observations where the phycocyanin to chlorophyll-a ratio $(\mathrm{PC} / \mathrm{CHL})$ is low $(<=0.1)$ are shown as blue dots while high $\mathrm{PC} / \mathrm{CHL}$ ratio $(>0.1)$ observations are displayed as red dots. 


\section{List of tables}

Table 2.1. Sources of in situ chlorophyll- $a$ data for the 37 lakes included in the in situ/satellite comparison. .20

Table 2.2. Mean MERIS-derived chl estimates (mg m-3) from the 2011 global snapshot for different size ranges. Number of lakes $=$ global number of lakes in that size category represented in the 2011 global snapshot.

Table 3.1. Data used to generate the latitude vs. light utilization index model. The reference for each lake study is shown on the table.

Table 3.2. Independent data used to validate the DIM model. The mean values for in situ observations and DIM retrievals are shown on the table .48

Table 4.1. Classification error matrix for $\mathrm{MCH}$ vs. in situ observations. $\mathrm{MCH}$ correctly classified 103 out of 118 comparisons for an overall accuracy of 87\% and Kappa of 0.74 .

Table 4.2. Classification error matrix for SSI vs. in situ observations. SSI correctly classified 37 out of 40 comparisons for an overall accuracy of $93 \%$ and Kappa of 0.83.

Table 5.1. Numbers of available in situ water quality samples measured in the western basin of Lake Erie within 3 days of a cloud-free SeaWiFS image collection during a cyanoHAB season between 1998 and 2007. There were no valid matchups in 2000 or 2001

Table 5.2. Classification error matrix for SeaWiFS-derived MCH vs. in situ observations. The hybrid algorithm agreed with 116 out of 143 in situ observations for an overall accuracy of $81 \%$ and Kappa coefficient of 0.57 .

Table 5.3. Linear regression statistics for the 20 -year time series mean and maximum $\mathrm{MCH}$ and SSI annual extents.

Table 5.4. $\mathrm{MCH}$ and SSI extent model equations and statistics. Extent models are derived using the weighted spring discharge metric $(w Q)$ as the predictor.

Table 6.1. Summary statistics for the four inherent optical properties (aph*665, PCLHap, SCDOM, $\gamma$ ) and laboratory biochemical parameters (Chl-a, PC, and FSS). Summary statistics include range, mean, and median values for each year and combined for both years 


\section{Preface}

Chapters 2,4,5,6 are composed of published material that can be found in peer-reviewed academic journals, and Chapter 3 is a work in preparation for publication as follows:

Chapter 2: Sayers, M. J., Grimm, A. G., Shuchman, R. A., Deines, A. M., Bunnell, D. B., Raymer, Z. B., ... \& Whitley, M. A. (2015). A new method to generate a highresolution global distribution map of lake chlorophyll. International journal of remote sensing, 36(7), 1942-1964. DOI: 10.1080/01431161.2015.1029099

Author Contributions: M. Sayers, R. Shuchman, and A. Grimm conceptualized and wrote the manuscript. D. Bunnell, A. Deines, M. Rogers, and D. Warner provided funding and conceptual feedback as program sponsors. Z. Raymer, C. Brooks, M. Whitley and A. Grimm processed remote sensing data. W. Woelmer, D. Bennion, and J. Mychek-Londer created and shared validation data sets.

Use Permission: Reproduction of this material is acceptable by Taylor \& Francis, which allows the author to use their article in a thesis or dissertation without requesting permission from the International Journal of Remote Sensing. Original article link: https://www.tandfonline.com/doi/full/10.1080/01431161.2015.1029099

Chapter 3. Sayers, M., Fahnenstiel, G., Shuchman, R., Laramie, K., Bosse, K., Almquist, Z. (2019). Global Carbon Fixation from Freshwater Lakes

Chapter 4: Sayers, M., Fahnenstiel, G. L., Shuchman, R. A., \& Whitley, M. (2016). Cyanobacteria blooms in three eutrophic basins of the Great Lakes: a comparative analysis using satellite remote sensing. International Journal of Remote Sensing, 37(17), 4148-4171. DOI: 10.1080/01431161.2016.1207265

Author Contributions: M. Sayers, G. Fahnenstiel, and R. Shuchman conceptualized and wrote the manuscript. M. Sayers and M. Whitley processed and analyzed data. M.Sayers processed satellite imagery and developed models.

Use Permission: Reproduction of this material is acceptable by Taylor \& Francis, which allows the author to use their article in a thesis or dissertation without requesting permission from the International Journal of Remote Sensing. Original article link: https://www.tandfonline.com/doi/abs/10.1080/01431161.2016.1207265

Chapter 5: Sayers, M. J., Grimm, A. G., Shuchman, R. A., Bosse, K. R., Fahnenstiel, G. L., Ruberg, S. A., \& Leshkevich, G. A. (2019). Satellite monitoring of harmful algal 
blooms in the Western Basin of Lake Erie: A 20-year time-series. Journal of Great Lakes Research, 45(3), 508-521. DOI: 10.1016/j.jglr.2019.01.005

Author Contributions: M. Sayers, R. Shuchman, and A. Grimm conceptualized and wrote the manuscript. G. Fahnenstiel contributed to manuscript review. M. Sayers, A. Grimm, and K. Bosse processed and analyzed data. S. Ruberg and G. Leshkevich provided data for model calibration and validation.

Use Permission: Reproduction of this material falls under the Creative Commons Attribution License, which states that anyone may copy, re-publish, and/or re-use the content provided that the original authors are clearly credited and The Journal of Great Lakes Reseaech is cited as the original publisher. See https://creativecommons.org/licenses/by-nc-nd/4.0/for details.

\section{Original article link:}

https://www.sciencedirect.com/science/article/pii/S0380133019300103

Chapter 6: Sayers, M. J., Bosse, K. R., Shuchman, R. A., Ruberg, S. A., Fahnenstiel, G. L., Leshkevich, G. A., ... \& Palladino, D. (2019). Spatial and temporal variability of inherent and apparent optical properties in western Lake Erie: Implications for water quality remote sensing. Journal of Great Lakes Research. DOI:

10.1016/j.jglr.2019.03.011

Author Contributions: M. Sayers, G. Fahnenstiel, and R. Shuchman conceptualized and wrote the manuscript. M. Sayers and K. Bosse processed and analyzed in situ optics data. S. Ruberg and T. Johengen provided funding for study. G. Leshkevich provided in situ calibration and validation data. D. Stuart collected in situ data. A. Burtner and D. Palladino processed all in situ laboratory samples.

Use Permission: Reproduction of this material falls under the Creative Commons Attribution License, which states that anyone may copy, re-publish, and/or re-use the content provided that the original authors are clearly credited and The Journal of Great Lakes Reseaech is cited as the original publisher. See https://creativecommons.org/licenses/by-nc-nd/4.0/for details.

\section{Original article link:}

https://www.sciencedirect.com/science/article/pii/S038013301930067X 


\section{Abstract}

Freshwater lakes are an important component contributing to ecosystem health and biodiversity on local, regional, and global scales. And while lakes only represent $<5 \%$ of the global surface area, they are often very productive systems which contribute significantly to carbon cycling dynamics and freshwater fish production on a number of spatial scales. Due to the remote location and sheer size of some of these lakes it has proven difficult to adequately document changes in water quality. Significant challenges exist to adequately monitor water quality, and in particular phytoplankton dynamics, over large spatial and temporal scales using traditional in situ methods. Satellite electro-optical remote sensing offers a potential tool to provide better characterization of phytoplankton dynamics for a variety of freshwater systems. This work resulted in an approach to quantify global summer phytoplankton abundance using a newly developed remote sensing derived chlorophyll-a product. This product was also used in conjunction with a newly created carbon fixation model to assess global freshwater phytoplankton production which provided new insights into the role freshwater systems play in the global carbon budget. Spatial and temporal assessments of specific populations of phytoplankton and cyanobacteria were established through the development of a new remote sensing algorithm to isolate high biomass assemblages in the Laurentian Great Lakes (Lake Erie, Lake Huron, Lake Michigan). The algorithm was developed to facilitate the fusion of multiple remote sensing data sources (SeaWiFS and MODIS) in order to generate a new 20-year time-series data product to better understand the factors controlling bloom dynamics. Finally, a spatio-temporal analysis documenting the variability of inherent optical properties (IOPs) in Lake Erie established a seasonal progression of phytoplankton/cyanobacteria community structures for two years over the vegetative season, the findings of which are critical for the development of the next generation of hyperspectral remote sensing algorithms to improve phytoplankton community characterizations from space. These documented results clearly show the utility of electro-optical remote sensing to provide characterization of phytoplankton dynamics and insights at both community and population scales in freshwater systems. 


\section{Introduction}

Freshwater lakes are an important component contributing to ecosystem health and biodiversity on local, regional, and global scales (Larsen et al. 2011; Moss et al. 2011; Bennington et al. 2012; Regnier et al. 2013). And while lakes only represent $<5 \%$ of the global surface area (Verpoorter et al. 2014; Downing et al. 2006), they are often very productive systems which contribute significantly to carbon cycling dynamics on a number of spatial scales (Cole et al. 2007). Moreover, many freshwater lakes throughout the world have been experiencing rapid changes in water quality due in part to climate change and anthropogenic forcing (Adrian et al. 2009, Revenga et al. 2005, Vitousek et al. 1997, Strayer 2010). Because of the multi-scale and multi-discipline importance of freshwater lakes, better understanding of their underlying dynamics and variability is desired.

Unfortunately, the distribution and size of lakes is not uniform across the globe. For example, the vast majority of freshwater is located in the northern hemisphere, particularly in the hard to reach boreal and arctic latitudes, while in the southern hemisphere lake concentration is low due to a much lower continental area (Lehner and Doll, 2004, Verpoorter 2014). Furthermore, despite the fact that there are approximately 117 million freshwater lakes (Verpoorter et al. 2014), the ten largest lakes contain over $50 \%$ of the world's surface freshwater by volume and area (Herdenforf et al. 1982). Moreover, the largest 1000 freshwater lakes contain $>95 \%$ of the world's freshwater and represent $>95 \%$ of the world's freshwater surface area (Herdendorf et al. 1982). Because of the global freshwater distribution complexity, it is not feasible to establish rigorous monitoring of the world's freshwater to provide assessments of water quality variability in light of climate change and other anthropogenic forcing. However, because of the dominance of freshwater area by large lakes it is perhaps feasible to link observable changes in those waters to global environmental perturbations and extrapolate to smaller systems.

In all freshwater environments phytoplankton form the foundation of all aquatic food webs by providing energy to higher order consumers all the way up to the largest fish and humans 
(Pomeroy 1974). Aquatic food webs are carefully balanced to ensure there is enough energy to support the diverse set of species and sizes of fishes that humans rely on for subsistence and recreational fishing. Freshwater phytoplankton abundance has been shown to correlate significantly with freshwater fish production (Deines et al. 2016), which is an important parameter for subsistence, commercial, and recreational fisheries worldwide (Welcomme 2011). Additionally, through the process of photosynthesis, phytoplankton consume carbon from the atmosphere transferring it on to other aquatic layers through grazing or sequestering it to the bottom. The significant reduction in atmospheric carbon by phytoplankton is very important to maintaining global climates suitable for human life. A healthy balance of freshwater phytoplankton abundance is important to assure the longterm sustainability of aquatic ecosystems and maintenance of regional and global carbon cycles.

Not all freshwater phytoplankton are desirable or healthy for aquatic ecosystems. Large blooms of phytoplankton, which can sometimes generate toxins harmful to humans and animals, can cause significant problems for ecosystems relied upon as a source of fresh drinking water, recreation, and subsistence fishing (Rinta-Kanto et al. 2005, Ouellette et al. 2006). Due in part to significant increases in regional temperatures and necessary nutrients, it is believed the occurrence of harmful algal blooms (HABs) are increasing worldwide (Otten and Paerl 2011). Understanding the fluctuations of these blooms for a given system can help establish cause and effect relationships between occurrences and driving factors with the goal of mitigating extreme events (Michalak et al. 2013).

Typically, phytoplankton communities are sampled from boats using a variety of water sampling equipment. For small lakes these samples are taken from small boats or even docks or shore. In these systems phytoplankton can be sampled on daily/weekly scales (Wetzel et al 1983, Reynolds 2006) However, for large lakes sampling of phytoplankton communities usually involves the use of large ships and less frequent sampling. Because ships are expensive and more difficult to operate, routine sampling in large lake is limited both temporally and spatially. For example, for the largest and most thorough synoptic 
cruises of the Great Lakes conducted in the 1970s, typically 5-7 cruises, were conducted during the year and 30-60 stations were sampled (Vollenweider et al. 1974). Under the best assumptions, a single station might represent anywhere between 500 and 1000 square kilometers for a 1-2-month period. Furthermore, the US EPA routine Great Lakes monitoring program consists of two cruises per year (Spring and Summer) where 15 sites on average are sampled per lake none of which represent the nearshore environment. And while these sampling schemes are spatially and temporally limited, they represent some of the most rigorous monitoring of any lake or set of lakes in the world.

Because phytoplankton dynamics are highly variable at the community level (global abundance) and the population level (functional group abundance) in situ monitoring on limited spatial and temporal scales do not allow us to fully capture the variability needed to better understand factors controlling changes. One technique that allows for the possibility of frequent temporal and spatial sampling on large lakes is remote sensing. Remote sensing offers the unique ability to observe water bodies which are difficult to sample, as well as providing rapid systematic revisit time to capture changes on short timescales. Remote sensing of aquatic systems is challenging and is just beginning to be readily used in freshwater environments. It is still not fully understood if remote sensing can be routinely used to accurately estimate water quality parameters, including phytoplankton abundance, for many different freshwater systems throughout the world (Mouw et al. 2015, Palmer et al. 2015).

Satellite remote sensing of phytoplankton biomass has been successfully demonstrated for Case I waters of the global ocean (O'Reilly et al 1998, 2000) where the reflected spectral radiance is a function of only phytoplankton abundance and seawater. Phytoplankton primarily control radiance upwelling from the water surface through the specific light absorption processes of the photosynthetic chlorophyll-a pigment bound within each cell. Chlorophyll-a strongly absorbs light in the blue $(\sim 440 \mathrm{~nm})$ and red $(\sim 667 \mathrm{~nm})$ portions of the Electro-Optical (EO) spectrum thus resulting in very low reflected energy in these spectral regions and greater reflectance in other regions (e.g. green). These characteristics 
in conjunction with the weak absorption by water in the blue region suggest changes in reflectance from blue-to-green are proportional to chlorophyll-a concentration.

O'Reilly et al. (1998) developed an empirical approach relating the ratio of blue-to-green reflectance to chlorophyll concentration using a fourth order polynomial. This approach has been adopted as the NASA standard method (OCx) for estimating marine chlorophylla concentration from ocean color satellite sensors. This method assumes other water color producing agents, such as dissolved organic matter (CDOM), co-vary with phytoplankton abundance resulting a change in reflectance that is still proportional to the chlorophyll-a concentration. These assumptions meet the definition of Case I water (Morel and Prieur 1977).

Freshwater systems are generally classified as Case II optically complex waters with multiple optically active constituents (OACs) that are not assumed to co-vary with chlorophyll-a concentration (Morel and Prieur 1977). Case II waters often include significant concentrations of CDOM and other non-algal particles (NAP) comprising of inorganic sediment particles and detrital material. These OACs have unique spectral absorption and scattering properties differing from chlorophyll-a which result in unique water leaving spectral reflectance profiles under variable concentrations of each OAC. Terrestrial inputs of CDOM and NAP are a distinguishing factor of freshwater and coastal ocean systems that fluctuate temporally depending on meteorological and environmental forcing. Understanding the optical characteristics of potentially complicated mixtures of OACs is the key for development and application of remote sensing algorithms in freshwater systems.

The optical characteristics and abundance of OACs relate directly to the amount of light reflected by the water and can thus be estimated from remote sensing using radiative transfer modeling (Mobley et al. 1994). Spectral reflectance is a function of total absorption and total backscatter in each wavelength and is most commonly expressed as: 


$$
R=\frac{f}{Q}\left(\frac{b_{b}}{a+b_{b}}\right)
$$

Where $\mathrm{R}$ is reflectance $\left(\mathrm{sr}^{-1}\right)$, a is absorption $\left(\mathrm{m}^{-1}\right)$, and $\mathrm{b}_{\mathrm{b}}$ is backscatter $\left(\mathrm{m}^{-1}\right)$, and $\mathrm{f} / \mathrm{Q}$ describes the bidirectional reflectance factor. The form of this equation is known as the Quasi Single Scattering Approximation (QSSA) (Gordon and Brown 1973) and is a derivation of the single scattering albedo.

The total absorption, a, can be partitioned into its components and is expressed as:

$$
\mathrm{a}(\lambda)=\mathrm{a}_{\mathrm{w}}(\lambda)+\mathrm{a}_{\text {phy }}(\lambda)+\mathrm{a}_{\text {nap }}(\lambda)+\mathrm{a}_{\mathrm{cdom}}(\lambda)
$$

where $\mathrm{a}_{\mathrm{w}}$ is absorption of pure water, $\mathrm{a}_{\mathrm{phy}}$ is the phytoplankton absorption coefficient, $\mathrm{a}_{\text {nap }}$ is the non-algal absorption (nap) coefficient, and $\mathrm{a}_{\text {cdom }}$ is the CDOM absorption coefficient. The total backscatter coefficient, $b_{b}$, is expressed as:

$$
b b(\lambda)=b_{b w}(\lambda)+b_{b p h y}(\lambda)+b_{\text {bnap }}(\lambda)
$$

where $b_{b w}$ is backscatter of pure water, $b_{b p h y}$ is the backscattering coefficient for phytoplankton, and $b_{\text {bnap }}$ is the non-algal particle backscattering coefficient.

These absorption and backscatter coefficients are known as inherent optical properties (IOPs) and can vary depending on factors such as phytoplankton size and structure, CDOM molecular weight, and sediment particle size and shape (Morel and Prieur 1977). These properties can be measured with in situ optical instruments and in the laboratory. The above equations imply the spectral shapes and magnitudes of the absorption and backscatter coefficients control the spectral shape and magnitude of reflectance with spectral shape predominantly influenced by phytoplankton pigment absorption. Because of the inherent linkage between IOPs and reflectance, Quantification of IOP variability is a crucial step in the creation of freshwater remote sensing algorithms.

Relatively recent advancements in the understanding of freshwater optical properties have led to the development of water quality remote sensing algorithms in select freshwater 
environments (Mishra et al. 2017). Both empirical and semi-analytical algorithms exist for freshwater systems and generally incorporate some level of regional tuning of coefficients derived from optical measurements made in the observed water body (Mishra et al. 2017). A specific example of these advancements are from the Laurentian Great Lakes where sophisticated bio-optical models (Color Producing Agent Algorithm, CPA-A) have been developed (Shuchman et al. 2006, 2013) to estimate concentrations of chlorophyll-a, CDOM, and total suspended matter from multi-spectral satellite data. These remote sensing derived data sets in conjunction with an empirically parameterized mechanistic phytoplankton production model (Fee 1973, Lang and Fahnenstiel 1996) were used to document trends in primary production for the post-dreissenid mussel period in Lakes Superior, Michigan, and Huron (Fahnenstiel et al. 2016). Additionally, Binding et al (2012) and Wynne et al (2008) have applied spectral shape methods targeting the quantification of high biomass phytoplankton and cyanobacteria blooms in the Great Lakes. And while these advancements have proven valuable for improved understanding of phytoplankton dynamics in the Great Lakes, limited research has been conducted to determine the transferability of this suite of algorithms, and underlying optical properties, to many different freshwater systems throughout the world.

A unique challenge in the application of optical remote sensing for freshwater is a lack of sensing platforms with spatial resolution capable of observing smaller lakes. Because water and accompanying particles are strongly absorbing, reflected light from the water volume is very low $(<5 \%)$ relative to other Earth system phenomena, particularly that of the overlying atmosphere. Because of the very weak reflectivity from water, highly sensitive instruments are required to sense variations in the water leaving signal brought upon by changing in water properties. To achieve this level of sensitivity, sensors integrate over large areas to ensure a sufficient accumulation of photons leaving the water volume are observed. Coarse spatial resolution $(\sim 1 \mathrm{~km})$ sensors such as SeaWiFS and MODIS possess the required sensitivity to monitor water color but are generally only applicable to the global ocean or the largest lakes. Advancements in sensor technologies have mitigated some of these issues and provide high quality observations of medium size lakes, however, 
with a tradeoff of less frequent overflights. These inherent sensor characteristics limit the degree to which bio-optical remote sensing is possible for small freshwater systems (Mouw et al. 2015).

In order to truly assess phytoplankton dynamics at the global and local scales a refined set of remote sensing algorithms and processing methodologies are needed. Currently there are no standard "lake color" products for freshwaters analogous to those ocean color marine products for which one could make time-series assessments. For lakes smaller than the world's 30 largest there are no standard atmospherically corrected reflectance products for use in remote sensing algorithms, they must be created manually per image costing a tremendous amount of labor. Because of the lack of attention paid to lake color remote sensing, assessments of EO data applications across broad scales for phytoplankton characterization are non-existent.

The main hypothesis of this dissertation is: Electro-optical (EO) remote sensing can be used to provide robust characterization and unique insights of phytoplankton dynamics at community and population scales in a variety of freshwater systems, particularly large freshwater systems.

In order to test this hypothesis, I developed a multi-faceted four-part approach that utilized satellite EO remote sensing data at different spatial and temporal scales to characterize phytoplankton dynamics. I defined the following specific research hypotheses to provide a framework to evaluate my overarching hypothesis.

1) Satellite remote sensing (RS) data and algorithms can be used to document global freshwater phytoplankton distributions and productivity (PP) in both large and small lakes.

2) Global freshwater phytoplankton abundance and PP varies by lake size and geographic location.

3A) At the population level (cyanobacteria) RS can provide time-series observations of phytoplankton bloom dynamics in large freshwater systems. 
3B) RS time-series observations can provide unique insights into factors controlling bloom dynamics in a large freshwater system.

4) Future improvement in the ability of EO RS to characterize phytoplankton dynamics requires critical IOP observations and more robust IOP based retrieval models. 


\title{
2 A New Method to Generate a Global Distribution Map of Lake Chlorophyll
}

\begin{abstract}
A new method was developed, evaluated, and applied to generate a global dataset of growing-season chlorophyll- $a$ (chl) concentrations in 2011 for freshwater lakes. Chl observations from freshwater lakes are valuable for estimating lake productivity as well as assessing the role that these lakes play in carbon budgets. The standard 4-km NASA OceanColor L3 chlorophyll concentration products generated from MODIS and MERIS sensor data are not sufficiently representative of global chl values because they can only resolve larger lakes, which generally have lower chl concentrations than lakes of smaller surface area. Our new methodology utilizes the 300-m-resolution MERIS Full Resolution Full Swath (FRS) global data set as input and does not rely on the land mask used to generate standard NASA products, which masks many lakes that are otherwise resolvable in MERIS imagery. The new method produced chl concentration values for 78,938 and 1,074 lakes in the Northern and Southern Hemispheres, respectively. The mean chl for lakes visible in the MERIS composite was $19.2 \pm 19.2$, the median was 13.3 and the interquartile range was 3.90$28.6 \mathrm{mg} \mathrm{m}^{-3}$. The accuracy of the MERIS-derived values was assessed by comparison with temporally near-coincident and globally distributed in situ measurements from the literature $\left(n=185, R M S E=9.39, R^{2}=0.72\right)$. This represents the first global-scale dataset of satellite-derived chl estimates for medium to large lakes.
\end{abstract}

Keywords: chlorophyll; lakes; MERIS; ocean colour satellite; monitoring

\subsection{Introduction}

Freshwater lakes are integral in the provisioning of ecosystem services and maintenance of biodiversity (Wilson and Carpenter 1999; Dudgeon et al. 2006). Though lakes, ponds and impoundments only represent on the order of 3-4\% of the global non-glaciated land surface (Verpoorter, Kutser, and Tranvik 2014; Downing et al. 2006), they are frequently highly 
productive, with disproportionately large net $\mathrm{C}$ fluxes compared to terrestrial systems (Cole et al. 2007). Lakes also provide habitat for freshwater fish - an important food source in many areas of the world (Welcomme 2011) - as well as other organisms representing an estimated 6\% of all described species (Dudgeon et al. 2006). Many freshwater lakes are currently undergoing rapid change due to climate shifts, anthropogenic perturbations, and introductions of non-native species (e.g. Ficke, Myrick, and Hansen 2007; Adrian et al. 2009; Revenga et al. 2005; Vitousek et al. 1997; Strayer 2010). Many of these lake changes can be directly or indirectly characterized by changes in colour-producing agents (CPAs), i.e., the biogeochemical constituents of the water column that affect the water's spectra, the primary CPAs being chlorophyll, suspended minerals, and coloured dissolved organic matter (Shuchman et al. 2013). Chlorophyll is of particular potential value as a proxy for trophic state, primary production and total ecosystem productivity (Lyche-Solheim et al. 2013). Thus, the capacity to monitor the water properties of freshwater lakes at a global scale via ocean colour satellites on an annual and intra-annual basis would provide important information to researchers, resource managers, and environmental regulators.

Relative to the open ocean (case 1 waters), where water colour is strongly dominated by the concentration of phytoplankton (Yentsch 1960), it is much more difficult to accurately estimate chlorophyll concentrations from multispectral imagery for coastal and inland (case 2) waters, where water colour is influenced by multiple CPAs that exhibit independent and considerable variations (Doerffer, Sorensen, and Aiken1999; Morel and Prieur 1977). Added to this, many freshwater lakes are at least partly optically shallow, meaning that the water is shallow enough that the contribution of radiance reflected by the lake bottom to the light detected by ocean colour sensors is potentially significant. Numerous techniques for remote sensing of water quality parameters in inland waters have recently been proposed, which are newly reviewed by Palmer, Kutser, and Hunter (2015). In brief, these inland techniques can be roughly categorized into two groups. The first group, semi-analytical bio-optical inversion models, were developed for the coastal ocean (Lee, Carder, and Arnone 2002; Maritorena, Siegel, and Peterson 2002) and inland waters (Shuchman et al. 2013; Korosov et al. 2009) and frequently rely on hyperspectral imagery 
and require tuning to local/regional inherent optical properties (IOPs) for accuracy, making them difficult to scale up to the global level (IOCCG 2000, 2006). The second group is comprised of empirical approaches, including band ratio algorithms (O'Reilly et al. 1998; O'Reilly et al. 2000; Gons, Rijkeboer, and Ruddick 2002; Binding, Greenberg, and Bukata 2012), which statistically relate the remotely sensed signal directly to chl (see review by Matthews 2011). Due to the optical complexity of inland waters described above, many researchers have presumed that an empirical approach general enough for application at the global level would not be sufficiently accurate to provide useful information on inland waters (Bukata 2005).

Various empirical ocean colour chlorophyll algorithms using satellite data from the CZCS, SeaWiFS, MODIS, MERIS and VIIRS sensors have been used successfully to estimate chl concentrations for single large lakes $\left(100 \mathrm{~km}^{2}\right.$ or larger) or small groups of large lakes throughout the globe (Chavula et al. 2009; Wu et al. 2009; Odermatt et al. 2008; Vos et al. 2003; Lesht, and Barbiero, and Warren 2012). The chl estimates for these lakes are relatively robust, though frequently overestimating low chl concentrations and underestimating high ones (Stramska et al. 2003; Cota, Wang, and Comiso. 2004). The accuracy of these estimates is due in large part to the many pixels available for lake-wide averaging and the fact that large lakes have more ocean-like open pelagic zones and are therefore less dominated by terrestrial inputs of suspended materials other than phytoplankton that can have significant optical effects (Lesht, and Barbiero, and Warren 2012; Matthews 2011). The successful use of empirical ocean colour satellite data to estimate chl in smaller lakes (between 0.5 and $100 \mathrm{~km}^{2}$ ) is far less documented (but see Koponen et al. 2004; de Moreas Novo et al. 2006) and until now has more frequently used simulated data (e.g. Gitelson et al. 2009; Simis et al. 2007; Kallio et al. 2001).

The freshwater lakes of the world vary greatly in size, with the frequency of lakes increasing sharply with decreasing surface area (Vernpoorter, Kutser, and Tranvik 2014; Downing et al. 2006). The Global Lakes and Wetlands Database assembled by the World Wildlife Fund includes approximately 170,000 lakes with surface areas $>1 \mathrm{~km}^{2}$ (Lehner 
and Döll 2004), and recent estimates have increased this estimate to approximately 200,000 (Downing et al. 2006) or 350,000 (GLOWABO database, Vernpoorter, Kutser, and Tranvik 2014). These high numbers are in sharp contrast to the fewer than 20 lakes worldwide larger than $10,000 \mathrm{~km}^{2}$. Of currently available technologies, only empirical ocean colour satellite estimations make the monitoring of medium-sized and larger freshwater lakes feasible at a global scale. Even considering that most lakes smaller than 1 $\mathrm{km}^{2}$ are not resolvable by any existing ocean colour satellite, monitoring the hundreds of thousands of lakes $>1 \mathrm{~km}^{2}$ poses a significant technical challenge.

We proposed that empirical ocean colour satellite data can be used to generate estimates of the global distribution of chlorophyll concentrations in inland lakes with an accuracy sufficient to be useful for examining spatial patterns and temporal changes. To address this hypothesis, the utility and limitations of different ocean colour satellite products were evaluated, and a method was developed to derive chlorophyll estimates from MERIS data for as many lakes throughout the world as possible. This new method was validated by comparison with in situ chlorophyll measurements compiled from the literature and representing a wide range of lake sizes and locations. The method was then applied to generate a 1-km-resolution global estimate of chlorophyll concentrations for lakes in both the Northern and Southern Hemispheres during their respective growing seasons for the year 2011. We present a discussion of why standard NASA products are not sufficiently representative of global freshwater lakes for rigorous modelling activities, followed by a narrative of the development of an improved product. A comparison of the distribution of chl values from this more representative snapshot with those obtained from the standard OC4 MERIS chl product is provided. Additionally, we highlight some of the patterns observed in the global growing-season chlorophyll snapshot for the year 2011. 


\subsection{Methods}

\subsubsection{Global-scale estimation of inland lake chl concentrations}

In this section, we describe the need for a custom method to generate a global satellite chl product for inland lakes. First we summarize the limitations of available standard global products, followed by the rationale for selecting MERIS as the data source and a description of the MTRI method developed to produce the global snapshot product.

\subsubsection{Limitations of NASA standard products}

We began by reviewing the chlorophyll-a data products derived from MODIS Aqua and MERIS using the respective OC3 and OC4 blue-green band ratio algorithms, available as standard products from the NASA OceanColor data facility operated by the Ocean Biology Processing Group (OBPG, http://oceancolor.gsfc.nasa.gov). Although a number of methods could be used to estimate lake chlorophyll at the global scale, the NASA OC algorithms are one of the most well-known and thoroughly validated for estimating chlorophyll. The latest available data versions for each sensor were used (see http://oceancolor.gsfc.nasa.gov/WIKI/OCReproc.html for details). The multi-mission reprocessing described in detail at the referenced link has led to high consistency between the chl products from these different sensors. NASA has found that standard MERIS estimates tend to be lower than MODIS in eutrophic waters (chl concentration between 1 and $10 \mathrm{mg} \mathrm{m}^{-3}$ ), but without a robust in situ dataset, it is unclear which is more correct (Franz 2013).

Landsat imagery was not considered as an alternative to ocean colour sensors, despite the advantage of 30-m pixel resolution, for several reasons including the lack of a robust globally applicable chlorophyll retrieval algorithm (non-optimum spectral bands for chlorophyll retrieval), the absence of an efficient atmospheric correction approach, and the much less frequent satellite revisit time (16 days vs. $\sim$ daily for ocean colour platforms). 
Apart from these issues, a global Landsat dataset would pose a significantly higher computational burden.

We first evaluated the 4-km Level-3 reduced resolution standard mapped image (SMI) MODIS OC 3 and MERIS OC4 products, which are available as global layers composited over a range of time intervals, to determine how much information about global freshwater lakes could be obtained as a ready-to-use product. As expected, we found that the 4-km pixels limited the number of inland lakes for which chl could be estimated and the amount of information that could be extracted for those lakes. The 4-km version of the entire mission composite for each sensor, representing the maximum number of lakes resolvable with these data products, contained pixels with valid chl values for only 866 lakes for MODIS and 923 lakes for MERIS. Thus, a method for producing a finer-scale global freshwater product from a lower OC product level was necessary.

\subsubsection{Sensor selection}

Initially, we planned to custom composite the daily Level-2 MODIS OC3 chlorophyll product (1-km resolution) into a global-scale 1-km map. Late in fall 2013, however, the 300-m MERIS Full Resolution Full Swath (FRS) global data set became available for use through the NASA-ESA data sharing collaboration. We compared the utility of this newly available dataset to the Level-2 MODIS product for a sample area in the Canadian Shield in terms of the amount of surface water for which chl values were retrieved. We found that MERIS imagery retrieved approximately $9 \%$ more water surface area than MODIS due to MERIS's smaller pixel size. In Figure 2.1, the green areas indicate the 4-km pixels retrieved by the MODIS L3 product, the yellow areas represent the gains in mapped area made by using a $1-\mathrm{km}$ custom composite rather than the $\mathrm{L} 3$ product, and the red areas indicate the further increase in retrievals achieved by utilizing MERIS rather than MODIS. 


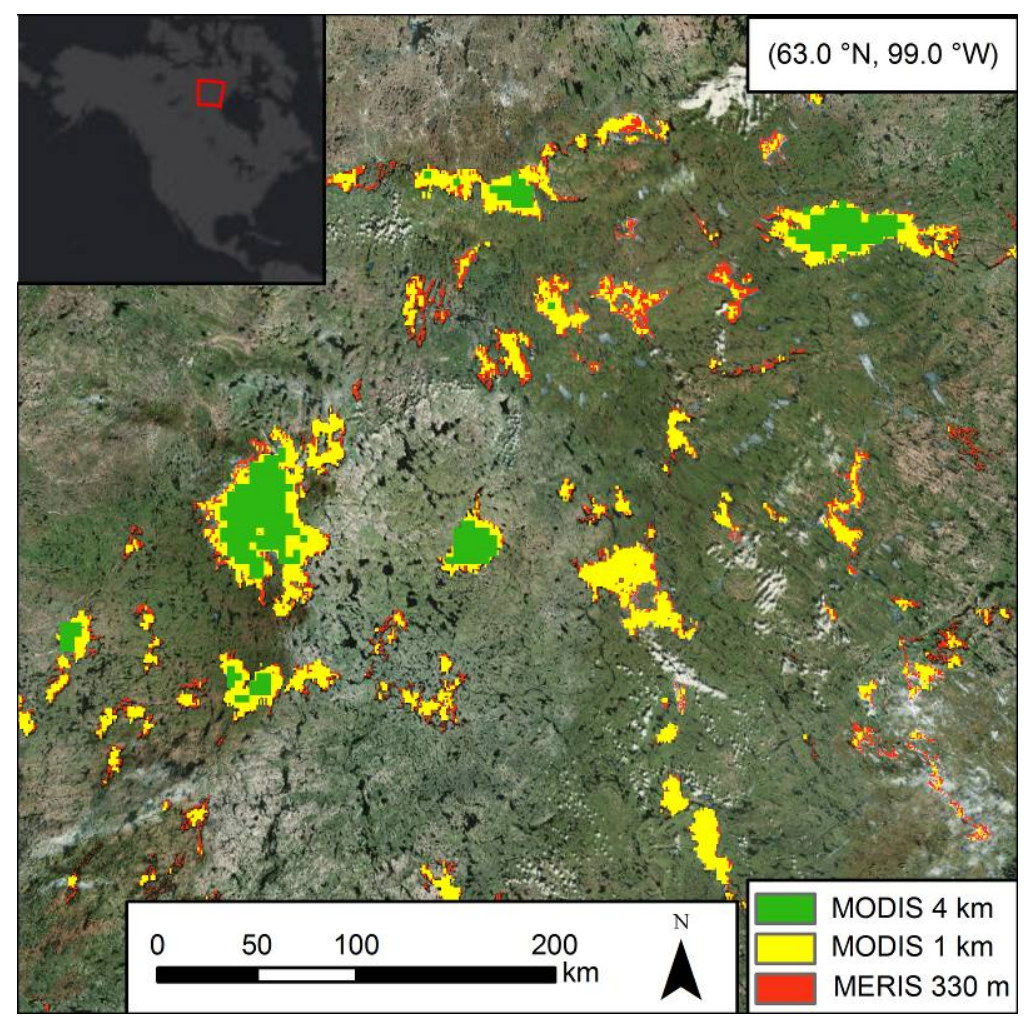

Figure 2.1. Example of the area of surface water retrieved by the MODIS L3 4-km product (represented in green) and the additional areas gained by using the MODIS 1-km composite (in yellow) and MERIS FRS imagery (in red) for an area in the Canadian Shield $\left(63.0^{\circ} \mathrm{N}, 99.0^{\circ} \mathrm{W}\right)$. MERIS retrieves the most information (green + yellow + red areas). The inset map at top left indicates the location of the example area.

Based on this increase in mappable lake area, it was decided to utilize MERIS as the "workhorse" ocean colour sensor for this global analysis because of the high perceived importance of minimum resolvable lake size and despite the fact that MERIS ceased to operate in early 2012. Figure 2.2 illustrates the relationship between lake surface area, the approximate global number of lakes in each size range as estimated by Verpoorter, Kutser, and Tranvik (2014), and satellite spatial resolution. These thresholds do not account for shoreline complexity, which made many lakes unretrievable with MERIS despite a surface area much larger than a MERIS pixel. Based on the relationship between lake size and 
satellite resolution shown in Fig. 2.2, the MODIS 4-km product would be expected to retrieve chl values for approximately 1000 lakes worldwide, which is similar to the value of 866 that we obtained in practice. The use of 1-km MODIS imagery provides information on a substantially larger estimated number of lakes, approximately 19,000 worldwide based on the Fig. 2.2 relationship. The even finer-resolution MERIS data has the potential to map chl distribution for lakes $\geq$ approximately $1 \mathrm{~km}^{2}$, numbering approximately 170,000 in the GLWD shapefile, though as noted, a substantial fraction of those were determined to be unretrievable due to their complex shorelines (high shoreline development ratio). The findings reported here for MERIS will be highly relevant to the inland chl retrieval abilities of OLCI, a new ocean colour sensor based on the design of MERIS, which is part of the instrument package planned for the Sentinel-3 mission, with the first satellite scheduled for launch in 2015.

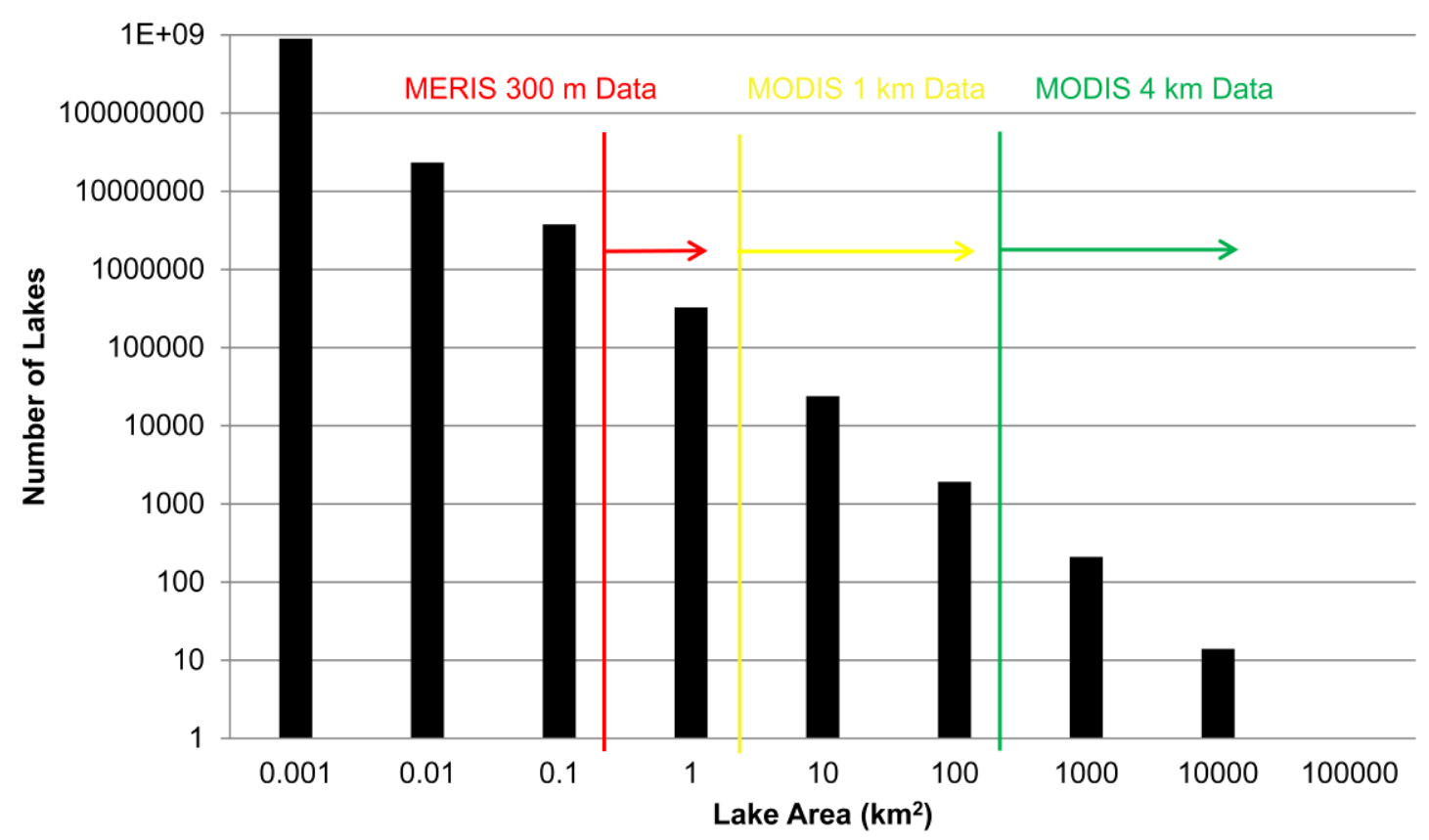

Figure 2.2. Log-log plot of lake size vs. global lake frequency, annotated with the minimum resolvable lake sizes for MODIS 4-km and 1-km composites and 
MERIS 300-m data. Column values derived from Verpoorter, Kutser, and Tranvik 2014.

\subsubsection{MTRI method and generation of global chlorophyll snapshot}

Figure 2.3 is a flow chart summarizing the steps of the MTRI method for generation of a global chlorophyll snapshot for the Northern and Southern Hemispheres, respectively, from MERIS OC4 data. These steps were necessary to retrieve the lakes that are not included in the L3 product due to both its coarser spatial resolution and issues with the NASA Ocean Discipline Processing System (ODPS) land/water mask (a global layer used to mask out non-water pixels in the production of all NASA Level 2 standard OC products). Reviewing examples of the standard Level 2 MERIS OC4 product, it was noted that chl estimates were not obtained for a surprising number of lakes for which we would expect chl to be retrievable by MERIS based on their shape and area. We determined that the land/water mask was masking out many otherwise valid inland chl pixel values. To retrieve these masked lakes, it was necessary to download the Level 1A versions of MERIS imagery for August and February 2011 for the Northern and Southern Hemispheres and process the L1A files to L2 using the SeaWiFS Data Analysis System software (SeaDAS, version 7.0) without using the ODPS land/water mask. August and February were selected to represent the growing season for the Northern and Southern Hemispheres, respectively, based on a preliminary analysis indicating that use of these months would yield the largest number of ice-free lakes and provide values representative of the vegetation growing season. In addition to not utilizing the standard land/water mask, we used ocean processing for all image pixels (both land and water). The L2 data processed in-house were then averaged for a global 330-m grid, dividing the globe into 30-degree tiles to alleviate the computational burden. Chl retrieval values less than 0.15 or greater than $100 \mathrm{mg} \mathrm{m}^{-3}$ were masked, as concentrations beyond these thresholds are outside the range of values for which MERIS OC4 produces reliable estimates (Hu et al. 2012; NASA 2010). Finally, as shown in Fig. 2.3, the cells of the global grid were analysed using the GLWD database as 
the source of geographic information on lake shorelines to calculate mean chlorophyll values for retrievable lakes.

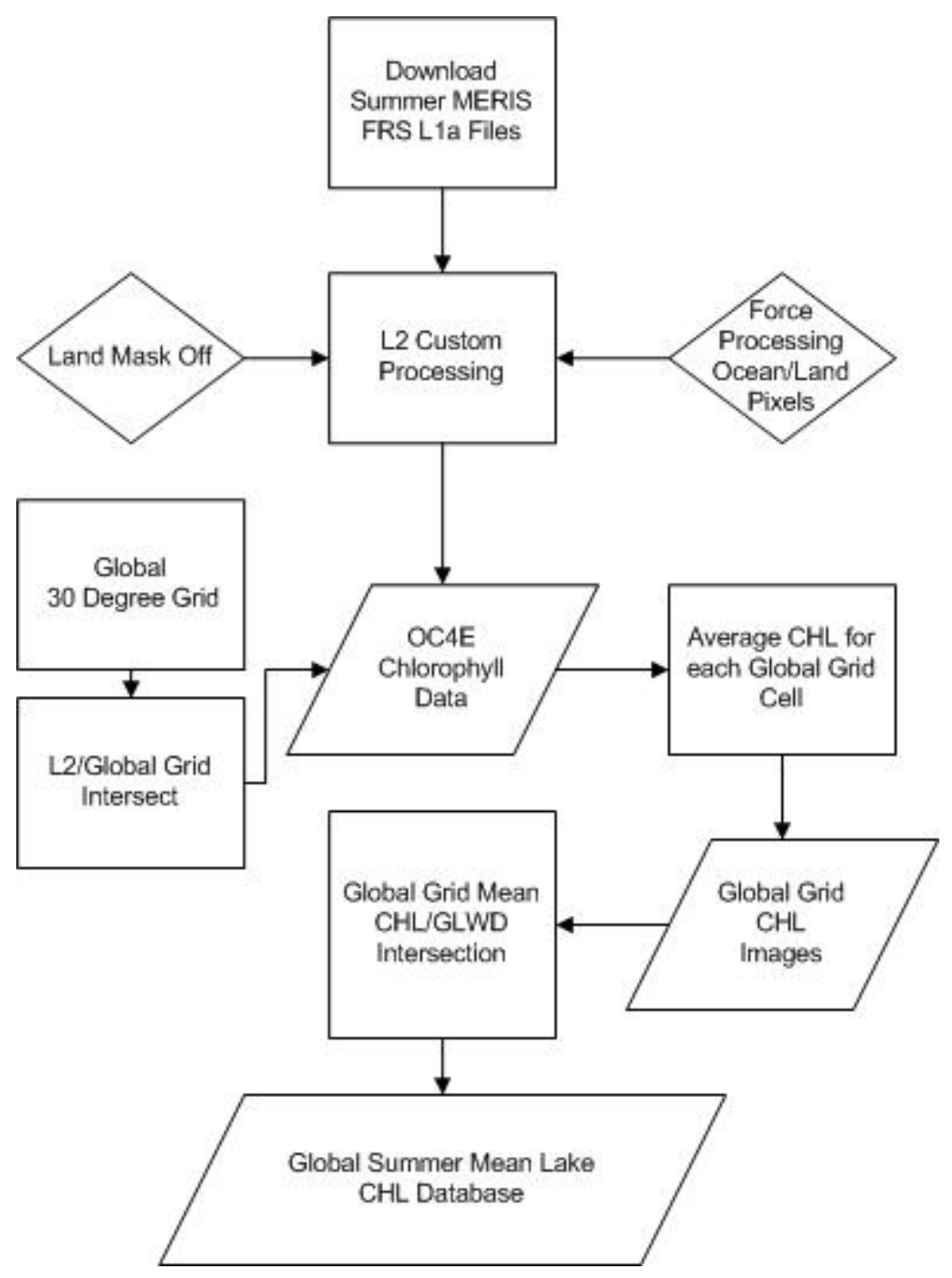

Figure 2.3. Steps involved in the MTRI method for processing MERIS OC4 chl data at the global scale.

Given the volumes of data involved in compositing daily MERIS images into a global product, a Wget-based tool (Niksic 1998) was developed to quickly download large amounts of OceanColor data via FTP for specified areas and date ranges. This was used to obtain imagery both for specific lakes and times to support the validation analysis and for 
all lakes during the selected summer 2011 months to produce chl estimates for a high number of lakes in a single recent year.

The Level 2 MERIS OC4 data processed in-house from L1A were composited for August 2011 for the Northern Hemisphere and for February 2011 for the Southern Hemisphere to create a "global summer" product representing growing season chl estimates for both hemispheres.

\subsubsection{Validation with in situ data and ancillary environmental information}

\subsubsection{Global in situ chl dataset}

In situ chlorophyll-a data were obtained by literature review (searching online databases using Google Scholar and ISI Web of Science), through direct contact with environmental agencies in North America, and from online databases (e.g., US Environmental Protection Agency's STORET water quality data repository; www.epa.gov/storet/). For inclusion in the validation data set, in situ lake chl data were required to represent a lake sufficiently large for MODIS and/or MERIS to obtain pixels uncontaminated by shoreline reflectance, correspond to dates when MERIS was operational (2002-2012), and represent a date or date range spanning 5 months or less. The threshold of 5 months was selected because values averaged over longer time-spans included too much seasonal variation for a useful comparison. Additionally, many reports provide in situ chl values for a calendar year without including information on the actual sampling dates used to generate those values, and such data are likely not comparable to a satellite composite of all cloud- and ice-free dates in that year. When in situ measurements were reported for multiple locations within a lake for a known timeframe, the average was taken to represent the whole lake. The lakes included in the validation dataset and the sources of the in situ chl data are summarized in Table 1. 
Table 2.1. Sources of in situ chlorophyll- $a$ data for the 37 lakes included in the in situ/satellite comparison

\begin{tabular}{lllll}
\hline & Lake Name & Latitude $\left(^{\circ}\right)$ & Longitude $\left(^{\circ}\right)$ & Source of in situ data \\
\hline 1 & Cass Lake & 47.4226 & -94.5476 & US EPA STORET, \\
& & & http://www.epa.gov/storet/dbtop.html \\
2 & Upper Red Lake & 48.1308 & -94.9147 & US EPA STORET \\
3 & Pelican Lake & 48.0620 & -92.9055 & US EPA STORET \\
4 & Center Hill Reservoir & 36.0053 & -85.7420 & US EPA STORET \\
5 & Barkley Reservoir & 36.8070 & -87.9585 & US EPA STORET \\
6 & Old Hickory Reservoir & 36.3092 & -86.1897 & US EPA STORET \\
7 & J. Percy Priest Reservoir & 36.0853 & -86.5517 & US EPA STORET \\
8 & Angostura Reservoir & 43.3048 & -103.4469 & US EPA STORET \\
9 & Lake Sinai & 44.2660 & -97.0780 & US EPA STORET \\
10 & Lake Poinsett & 44.5650 & -97.0802 & US EPA STORET \\
11 & Lake Kampeska & 44.9256 & -97.2102 & US EPA STORET \\
12 & Waubay Lake & 45.4247 & -97.4004 & US EPA STORET \\
13 & Sharpe Lake & 44.1688 & -99.7316 & US EPA STORET \\
14 & Bitter Lake & 45.2804 & -97.3084 & US EPA STORET \\
15 & Lake Francis Case & 43.1463 & -98.7786 & US EPA STORET \\
16 & Lake Michigan & 44.0135 & -86.7611 & US EPA STORET \\
17 & Lake Saint Clair & 42.4571 & -82.6774 & US EPA STORET \\
18 & Kootenay Lake & 49.5953 & -116.8172 & Schindler et al. 2003 \\
19 & Lake Taihu & 31.2003 & 120.1909 & Zhang, Qin and Liu 2007 \\
20 & Ramsagar Reservoir & 25.6685 & 78.3960 & Khondker et al. 2012 \\
21 & Lake Malawi & -11.9884 & 34.5110 & van der Sande et al. 2012 \\
22 & Lake of the Woods & 49.2410 & -94.6675 & Pla et al. 2005 \\
23 & Lake Champlain & 44.6126 & -73.3065 & US EPA STORET
\end{tabular}




\begin{tabular}{lllll}
\hline & Lake Name & Latitude $\left(^{\circ}\right)$ & Longitude $\left(^{\circ}\right)$ & Source of in situ data \\
\hline 24 & Lake Ladoga & 60.8311 & 31.4758 & Viljanen et al. 2008 \\
25 & Lake Baikal & 53.3998 & 107.6614 & Heim et al. 2005 \\
26 & Volgogradskoe Reservoir & 50.4187 & 45.6643 & Nemirovskaya 2012 \\
27 & Lake Calamus & 41.8681 & -99.2568 & US EPA STORET \\
28 & Lake Tana & 12.0117 & 37.3142 & Wondie et al. 2007 \\
29 & Amisk Lake & 54.5674 & -102.2345 & Alberta Lake Management Society 2008 \\
30 & Lake Chaohu & 31.5679 & 117.5312 & Yang et al. 2013 \\
31 & Toba Lake & 2.6069 & 98.8107 & Barus et al. 2008 \\
32 & Ir H Juanda (Djuanda) Reservoir & -6.5501 & 107.3367 & Garno 2003 \\
33 & Tonle Sap Lake & 12.8849 & 104.0722 & Campbell et al. 2006 \\
34 & Lake Apopka & 28.6237 & -81.6261 & US EPA STORET \\
35 & Lake Harris & 28.7775 & -81.8154 & US EPA STORET \\
36 & Funil Reservoir & -22.5525 & -44.6073 & Soares et al. 2009 \\
37 & Laguna de Rocha & -34.6188 & -54.2895 & Piccini et al. 2006 \\
\hline
\end{tabular}




\subsubsection{Global Lakes and Wetlands Database}

This study also made use of the most comprehensive available global-scale GIS database of lake and reservoir boundaries, the Global Lakes and Wetlands Database (GLWD) (Lehner and Döll 2004). The GLWD represents a compilation of numerous existing maps and datasets and has been validated as comprehensive for lakes $\geq 1 \mathrm{~km}^{2}$. GLWD Levels 1 and 2, representing approximately 250,000 permanent open water bodies with a surface area $\geq 0.1 \mathrm{~km}^{2}$, were combined, and the polygons included in these shapefiles were used as lake location input data to calculate whole-lake statistics from the MERIS OC4 raster layers.

\subsubsection{Ancillary environmental data}

To explore the regional characteristics of global freshwater chl revealed by the 2011 MERIS snapshot, various statistics were calculated for the snapshot values within zones corresponding to Abell et al.'s (2008) Freshwater Ecoregions of the World. Abell et al.'s boundaries divide the freshwater habitats of the globe into 426 units based on freshwater fish species distributions and major ecological patterns. A major objective of these comparisons was to determine whether, for lakes where chl cannot be retrieved with MERIS, chl could be estimated based on a regional average or correlation with climate variables.

\subsubsection{Matching in situ and satellite-derived chl data}

The MTRI method for generating global MERIS freshwater chl snapshots was evaluated using near-coincident and spatially collocated measurements of satellite and in situ data with the following guidelines. Daily satellite products were composited to represent the time period associated with a given in situ measurement as closely as possible. Where the in situ data were collected within one or a few days, the 8-day composite containing those dates was used to increase the probability of obtaining cloud-free pixels for more of the 
lake's area. Given the constraints on in situ data described in Section 2.2.1, 185 matches were made between in situ and satellite-derived estimates. These matches include 37 unique lakes (median of $2.5 \mathrm{obs} /$ lake) ranging in size from 1.5 to approx. $58,000 \mathrm{~km}^{2}$ and in latitude from $60.8^{\circ} \mathrm{N}$ to $34.6^{\circ} \mathrm{S}$. As indicated in Figure 2.4, the lakes used for the in situ/satellite comparison are concentrated in North America due to its comparative wealth of available in situ data.

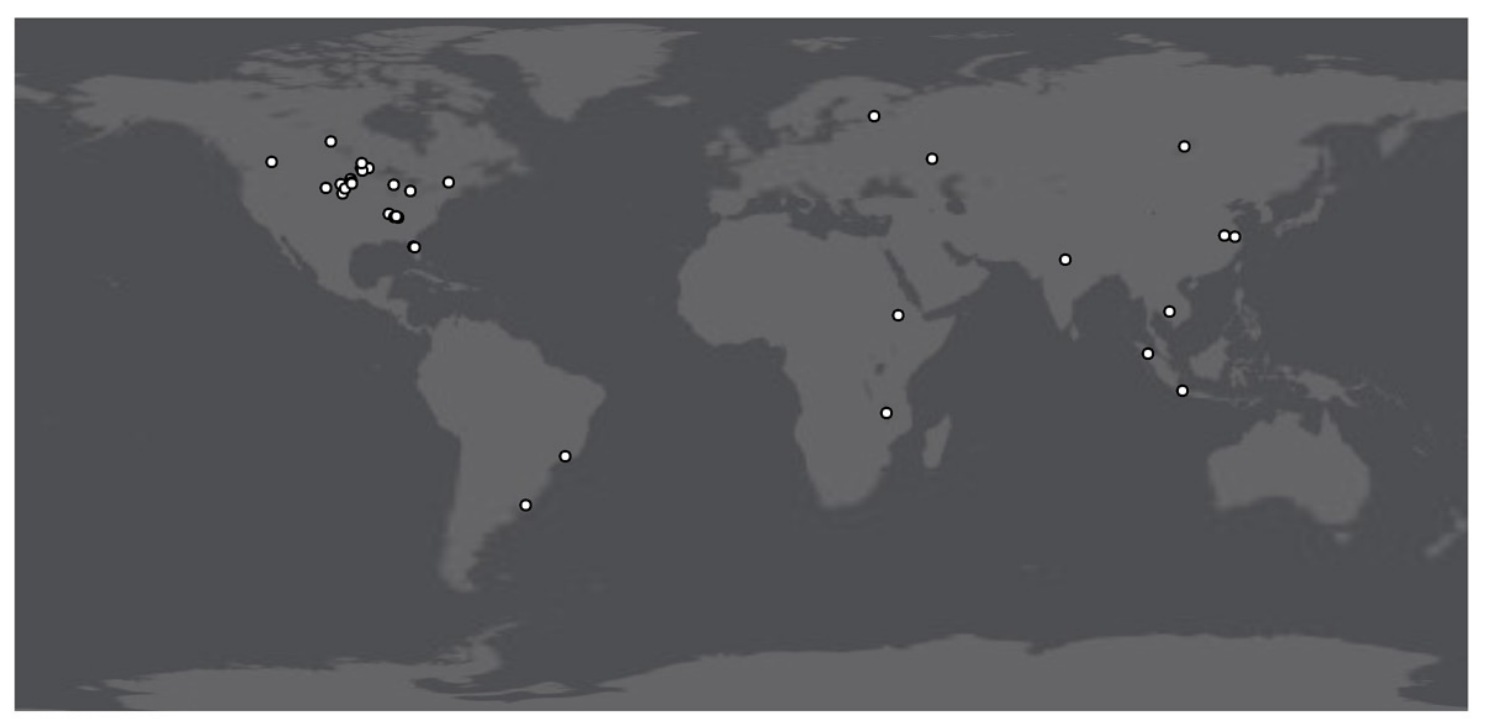

Figure 2.4. Locations of the 37 lakes for which in situ chlorophyll- $a$ data from one or more dates were compared to MERIS OC4 values for the same lake and time period.

\subsection{Results}

\subsubsection{MERIS satellite-derived vs. in situ chl estimates}

MERIS satellite imagery has been successfully used to retrieve reliable chlorophyll estimates in inland waters using a variety of algorithms. Many of the reported MERIS chlorophyll retrieval approaches use red and NIR reflectance to yield accurate estimates in turbid inland waters (Gower et al. 1999; Gons et al. 2002, 2009; Gitelson et al. 2008; Moses et al. 2009; Campbell et al. 2011) through empirically defined parameters for regionally 
specific waters. This regional parameterization can often lead to poor algorithm performance across wider ranges of global water bodies. In order to achieve a consistent level of accuracy for a global inland water chlorophyll snapshot, the standard MERIS OC4E algorithm was utilized. The algorithm has been shown to achieve consistent retrieval accuracy across a range of chlorophyll concentrations using the NOMAD 2 data set (http://oceancolor.gsfc.nasa.gov/REPROCESSING/R2009/ocv6/). Although it is acknowledged that tuned case II specific algorithms can achieve higher levels of accuracy locally, it is unclear how those accuracies vary on a global scale.

To characterize the algorithm's performance under the broader range of conditions represented by global inland lakes, we compared values generated using the MTRI method to the in situ data described in Fig. 2.4. The agreement between in situ and MTRI-processed MERIS chl values was reasonable considering the various potential sources of error involved in a global-scale analysis $\left(\mathrm{RMSE}=9.39, \mathrm{R}^{2}=0.72\right.$; Figure 2.5). The difference between in situ and satellite-derived chl values ranged from 0.04 to $35.0 \mathrm{mg} \mathrm{m}^{3}$. This difference tended to increase on the absolute scale and decrease on the log scale with the chl value (Figure 2.5). There was no meaningful relationship between the timespan of an observation (ranging from 1 day to 5 months) and in situ/satellite difference $\left(\mathrm{R}^{2}=0.01\right)$. Additionally, no interaction was observed between in situ/satellite difference and lake latitude. Our observed RMSE was similar to error values reported for comparable comparisons of in situ and MERIS chl data (Gons et al. 2002, 2005).

detail with the mask (below left) and without the mask (below right). 


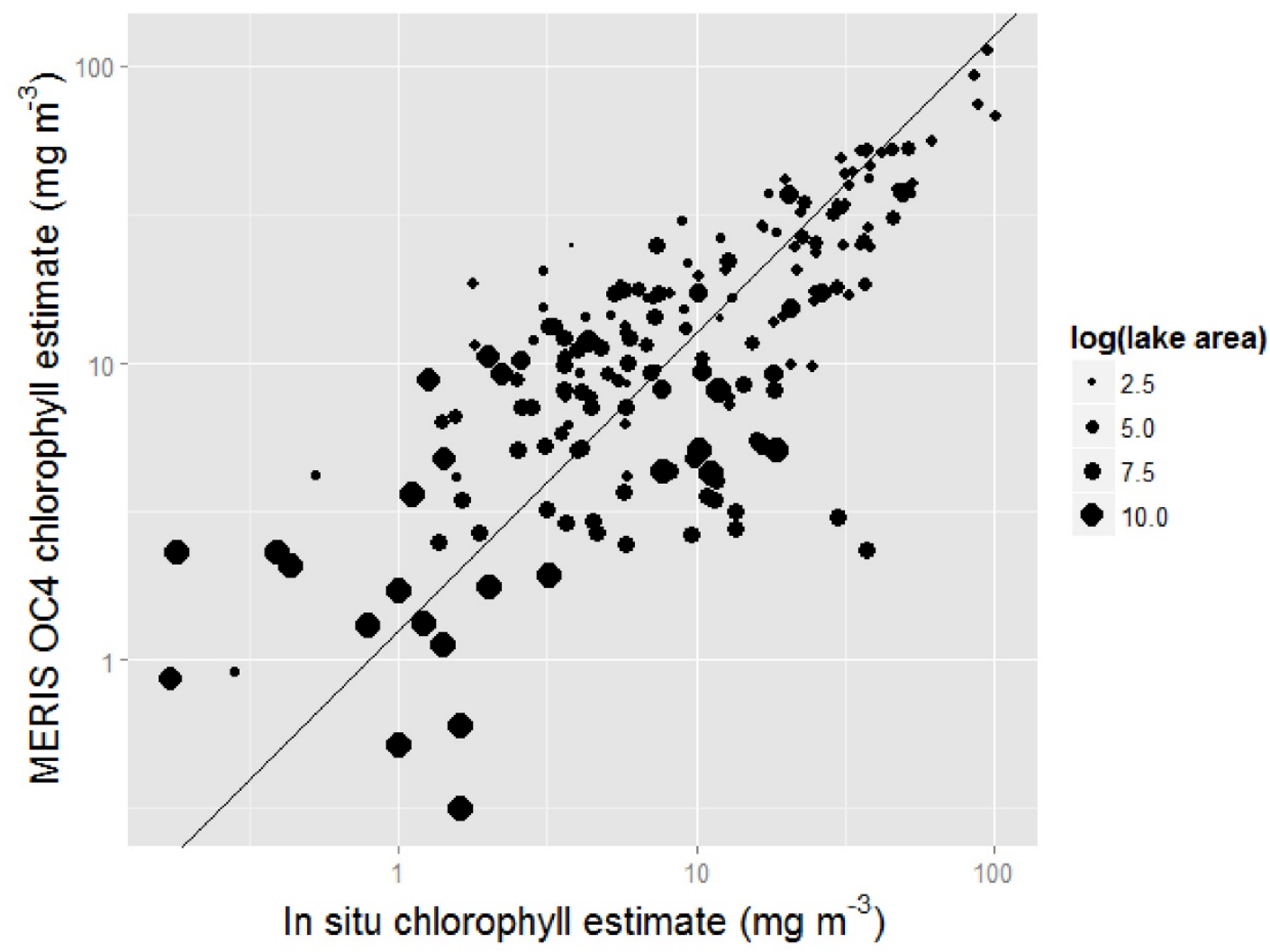

Figure 2.5. Comparison of chl values measured in situ vs. derived from MERIS ocean colour satellite imagery on a log-log scale ranging from 0 to 100 for 37 unique lakes distributed across the globe ( $\mathrm{n}=185$ observations). Point size corresponds to log lake surface area $\left(\mathrm{km}^{2}\right)$. Solid black line is identity line; $\mathrm{RMSE}=9.39, \mathrm{R}^{2}=0.72$.

Our validation yielded significantly higher errors compared to the NASA MERIS OC4E NOMAD 2 dataset validation (http://oceancolor.gsfc.nasa.gov/REPROCESSING/ R2009/ocv6/) due to several important differences between the two, which are discussed further in section 4.1. Based on the NASA OC4E validation diagnostics, the extensive previous investigations cited above, and our own global validation, we believe that MERIS OC4E's performance in estimating chl concentrations for inland waters makes it a suitable algorithm for this global lakes application. 


\subsubsection{Comparison of the new MTRI method and standard L2 product}

The water bodies resolvable from MERIS L2 imagery generated with and without the ODPS land/water mask (L2 Custom Processing, Fig. 2.3) were compared for a sample area in the Canadian Shield to quantify the area of surface water that became visible by omitting the ODPS mask (see Figure 2.6). Of the approx. 700 water bodies determined from GLWD data to be present within the red box in the top panel of Figure 2.6, 65 were resolved from Level 2 MERIS imagery using the ODPS land mask, whereas 634 were resolved without the mask. The bottom panels of Figure 2.6 provide a closer view (corresponding to the green box) of the difference between the results using the NASA land/water mask (left) and using no mask (right). Removing the land/water mask does result in chl values being produced for some pixels outside of water bodies, which accounts for some of the speckling seen in the right inset image, but because we used the GLWD data as "ground truth" for lake locations, this was not a concern for our purposes.

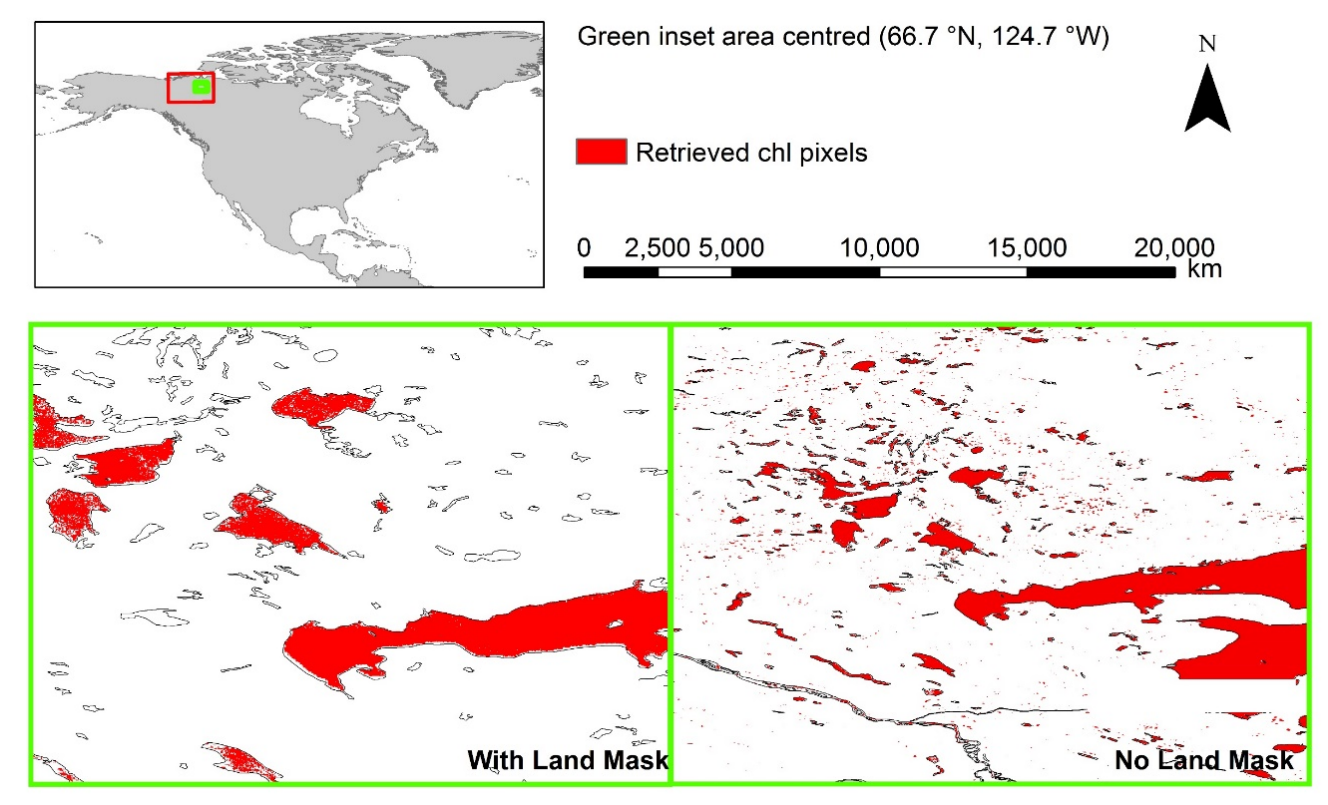

Figure 2.6. Comparison of chlorophyll estimates from MERIS L2 data for an area of the Canadian Shield (top panel). Of the $\sim 700$ water bodies present within the red box, 65 were resolved in an L2 image using the NASA ODPS land mask 
and 634 were resolved in the same image processed without the mask. For illustration, the area outlined in green in the top panel is shown in greater Description of 2011 snapshot data and global patterns

Using the new MTRI method with custom L2 processing on MERIS data, 1,822 MERIS scenes were composited into a global growing season snapshot for 2011. Chl values could be retrieved for over 11 million 330-m pixels, representing a total surface area of approx. $1,228,000 \mathrm{~km}^{2}$. The retrieved pixels represent 80,012 of the lakes defined in the GLWD shapefile (Fig. 2.7), in sharp contrast to the 923 GLWD lakes that can be resolved from the L3 4-km MERIS OC4 product and the estimated 58,000 lakes that could have been resolved using the new MTRI method on MODIS 1-km imagery. The lakes that could be estimated using this new MERIS product represent approximately one-third of the 246,957 lakes $\geq 0.1 \mathrm{~km}^{2}$ in the combined GLWD Levels $1 \& 2$ dataset. Figure 2.7 shows the distribution of lakewide chl values for the lakes represented in the 2011 MERIS snapshot. Approximately $7 \%$ of all lakes were estimated to have extremely low chl values of less than $0.5 \mathrm{~m}^{-3}, 28 \%$ had values of less than $5 \mathrm{mg} \mathrm{m}^{-3}, 42 \%$ of the lakes had mean values of less than $10 \mathrm{mg} \mathrm{m}^{-3}$, and $23 \%$ of all lakes had mean values exceeding $10 \mathrm{mg} \mathrm{m}^{-3}$. The mean chl for lakes visible in the MERIS composite was $19.2 \pm 19.2$, the median was 13.3 and the interquartile range was $3.90-28.6 \mathrm{mg} \mathrm{m}^{-3}$. 


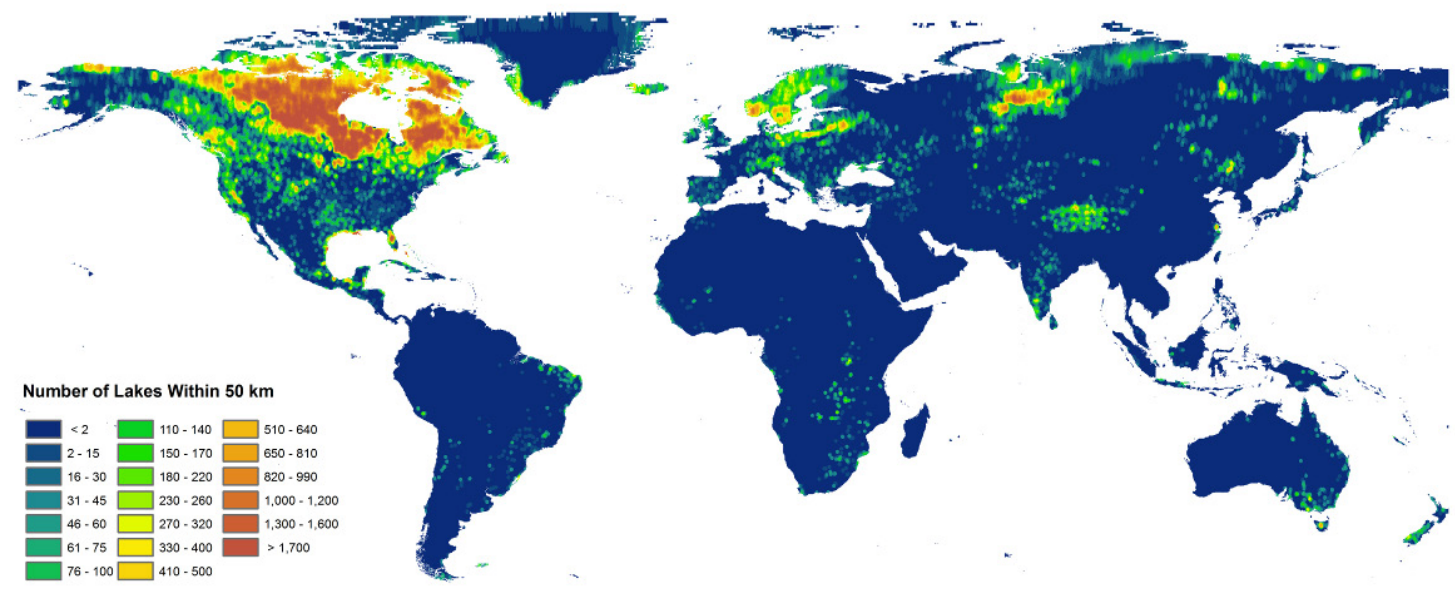

Figure 2.7. World distribution of the density of lakes from the GLWD shapefile that are visible in the MERIS 2011 global snapshot composite when the land mask was not used for data processing. Lake densities are represented as the number of lakes whose centre points fall within $50 \mathrm{~km}$ of the pixel (an area of approx. $7850 \mathrm{~km}^{2}$ ). Note that lakes are heavily concentrated around $40-70^{\circ} \mathrm{N}$.

Due to the uneven distribution of land on the Earth's surface, the lakes retrieved in the Northern Hemisphere vastly outnumber those visible in the Southern Hemisphere $(98.7 \%$ northern/1.3\% southern). Figure 2.9 illustrates this; the concentration of points at midnorthern latitudes with medium to low chlorophyll- $a$ values represents the chlorophyll- $a$ distribution as well as the uneven distribution of fresh water on the earth's surface. 


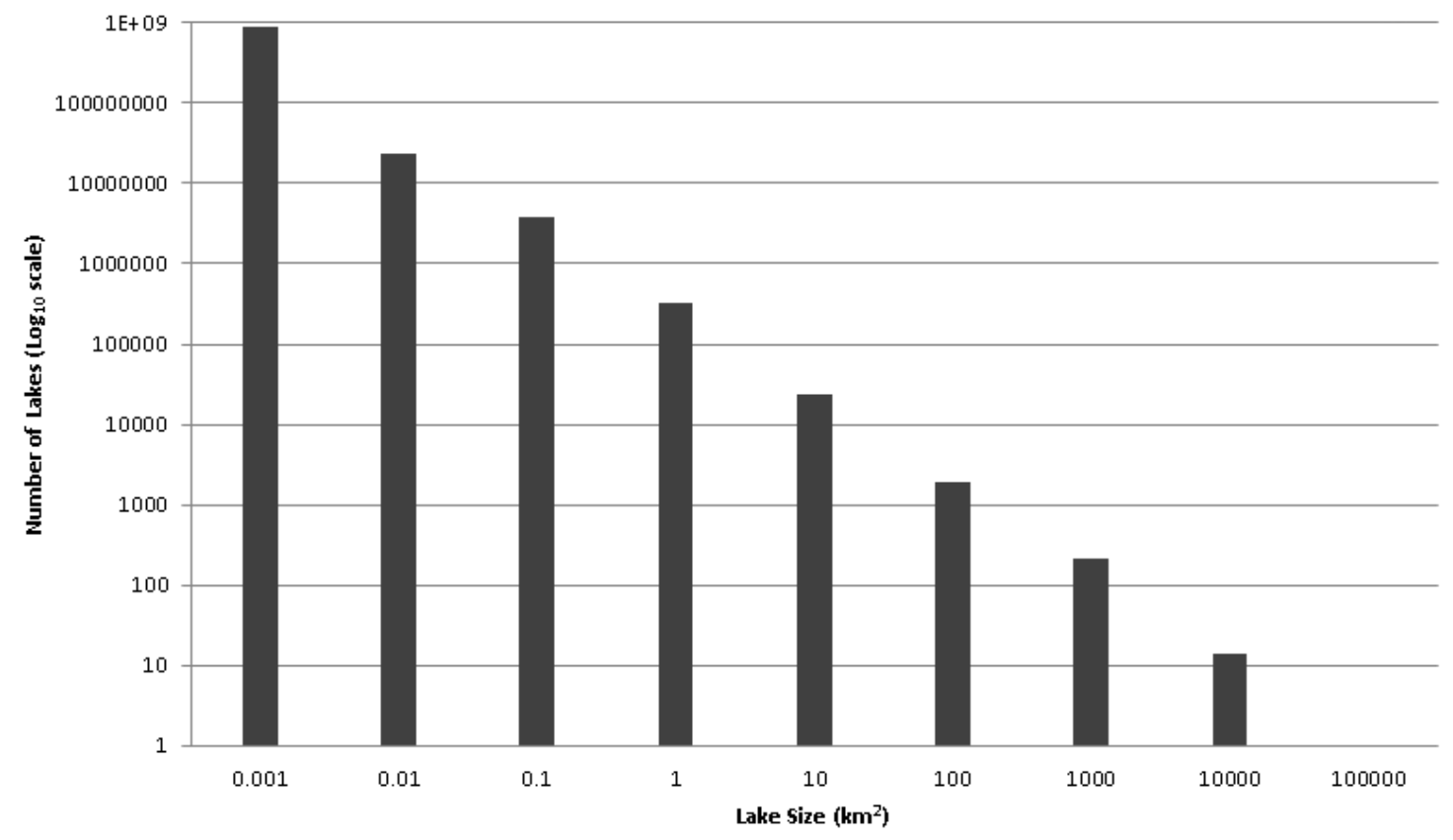

Figure 2.8. Histogram of mean lake-wide chl concentrations for the $\sim 80,000$ inland lakes from Global Lakes and Wetlands Database Levels 1 and 2 that could be retrieved with the MERIS 2011 global growing-season snapshot for world freshwater lakes. The mean chlorophyll-a value was $19.2 \pm 19.2 \mathrm{mg} \mathrm{m}-3$ and the median was $13.3 \mathrm{mg} \mathrm{m}$ 


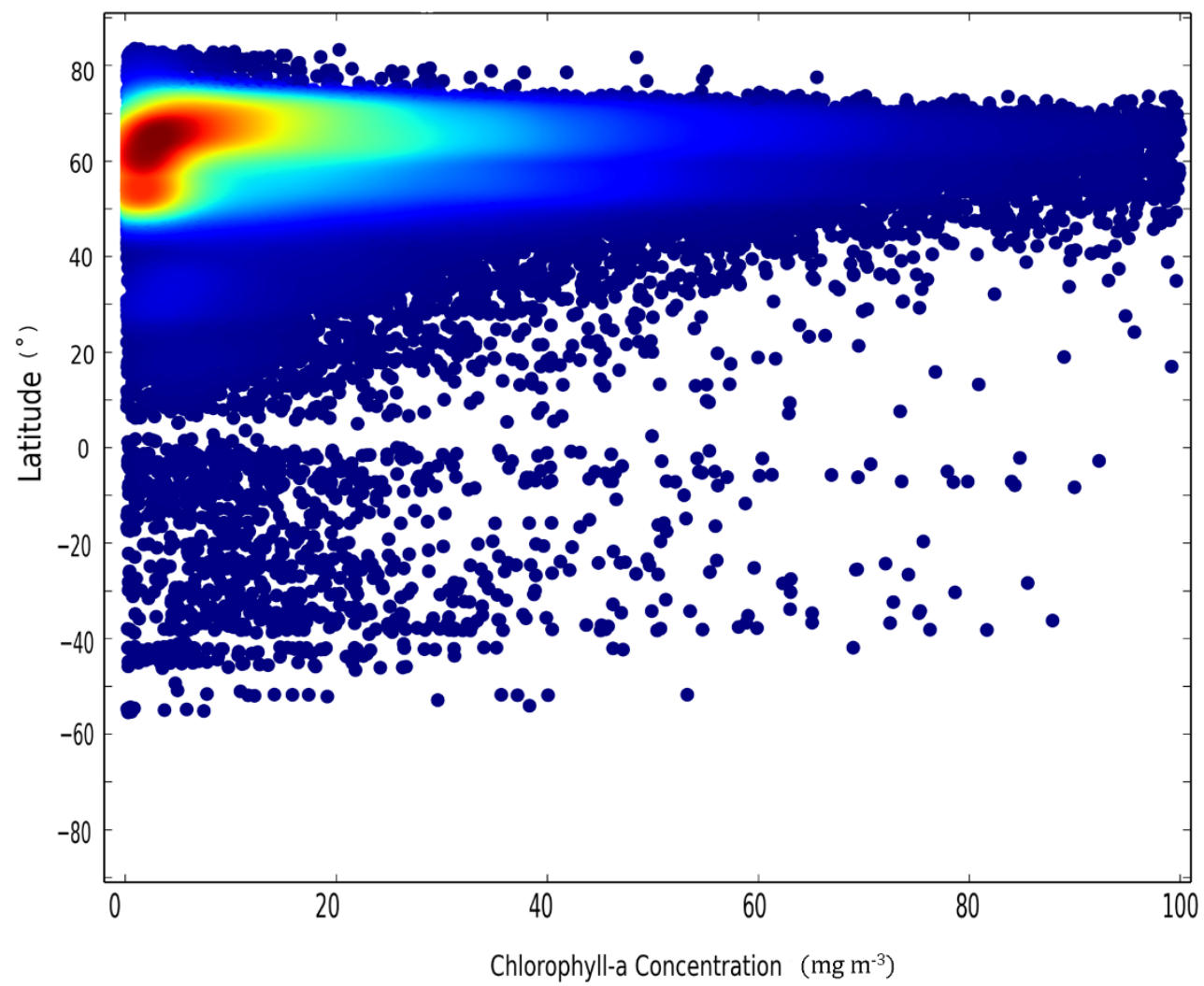

Figure 2.9. Density plot of mean lake-wide values of chl vs. the latitude of the lake's centre point for the approx. 80,000 lakes resolvable in the MERIS global growing season snapshot for the year 2011.

Table 2.2 below presents the mean MERIS-derived chl estimates for different lake surface area size ranges. Though there is a general trend of decreasing chl with increasing surface area, the mean values for the four smallest size classes are fairly similar, with much lower mean values for only the very large lakes. This similarity indicates that without information on the drainage basin / lake area ratio, lake size alone is not a strong predictor of chl concentration at the global scale. 
Table 2.2. Mean MERIS-derived chl estimates (mg m-3) from the 2011 global snapshot for different size ranges. Number of lakes $=$ global number of lakes in that size category represented in the 2011 global snapshot.

\begin{tabular}{lll}
\hline Surface area range $\left(\mathbf{k m}^{\mathbf{2}}\right)$ & $\begin{array}{c}\text { Mean MERIS chl } \\
\left(\mathbf{m g ~ m} \mathbf{~ m}^{\mathbf{3}}\right)\end{array}$ & Number of lakes \\
\hline$<1$ & 19.1 & 9,743 \\
$1-10$ & 19.5 & 58,087 \\
$10-100$ & 17.9 & 10,839 \\
$100-1,000$ & 17.0 & 1,205 \\
$1,000-10,000$ & 14.1 & 121 \\
$>10,000$ & 5.0 & 17 \\
\hline All lakes & 19.2 & 80,012 \\
\hline
\end{tabular}

\subsubsection{Comparison across ecoregions}

\subsubsection{Freshwater Ecoregions of the World}

In order to evaluate whether the chl concentrations of lakes not visible to MERIS could be reasonably estimated based on their ecoregion, we examined the variation in lake chl values within and among biogeographic regions by calculating the mean value and coefficient of variation within each of Abell et al.'s (2008) Freshwater Ecoregions of the World (FEOW). FEOW polygons are delineated based on hydrography and on distinct biotic assemblages, using freshwater fish as a proxy. Though mean chl varied considerably among FEOW ecoregions, values were also highly variable within regions for much of the world (Fig. 2.10). This high variability within ecoregions highlights the complexity of the factors determining the chl concentration of a given lake. Given these findings, the use of regional chl averages for further analysis does not appear to be very useful. 

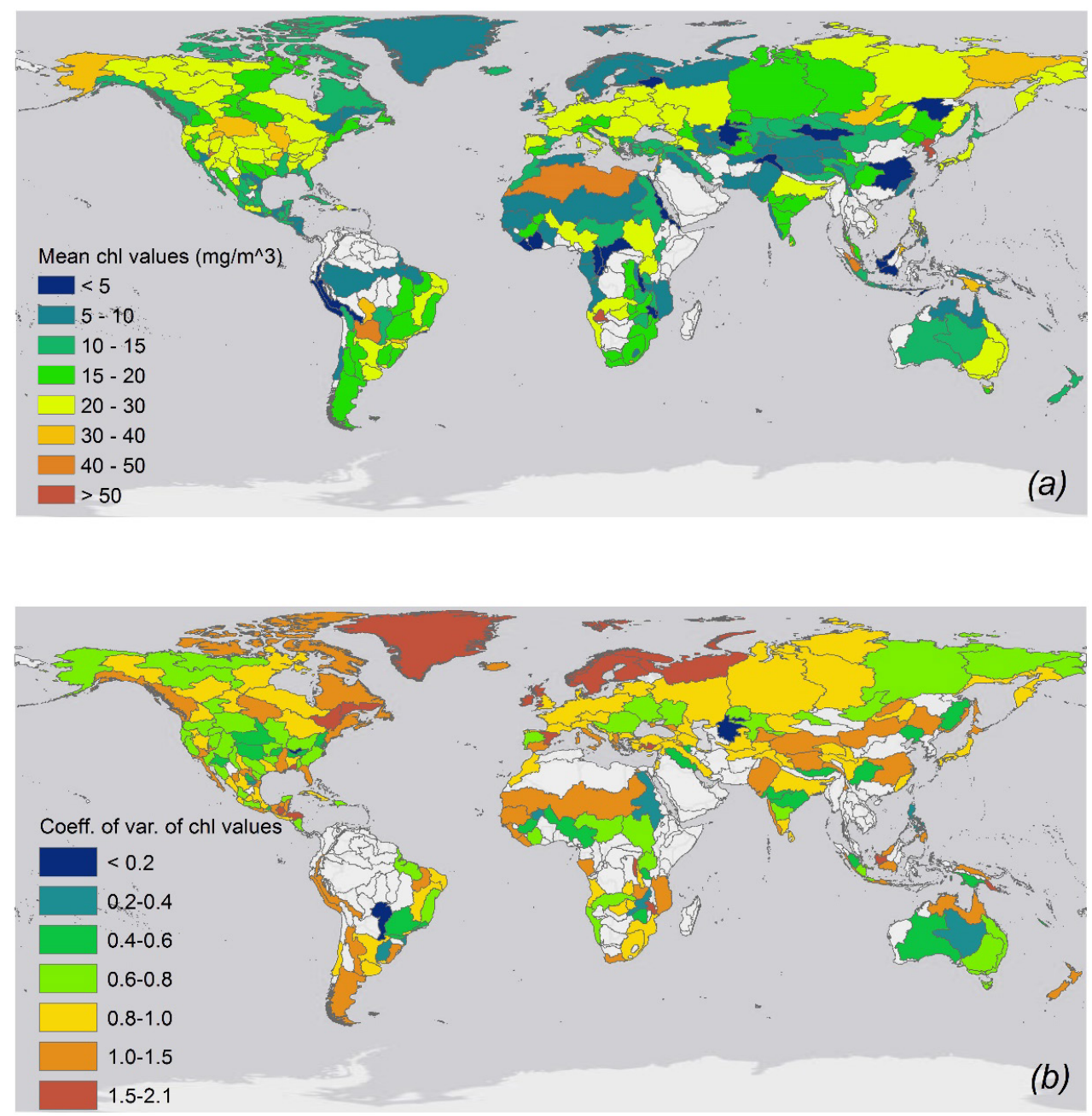

Figure 2.10. Mean MERIS-derived chl value $(a)$ and coefficient of variation $(\mathrm{CoV})(b)$ for each of Abell et al.'s (2008) Freshwater Ecoregions of the World. Light grey ecoregions include no retrieved lakes $(a)$ or fewer than three retrieved lakes $(b)$. The high $\mathrm{CoV}$ within many ecoregions indicates that lakes that are not retrieved by MERIS cannot be easily estimated based on their neighbors. 


\subsection{Discussion}

\subsubsection{Comparison to in situ data}

The comparison of in situ and satellite chl estimates revealed some patterns with lake characteristics. Abolute satellite chl estimates differed less from in situ values for larger lakes. In our validation that included 37 lakes across the globe, the absolute difference between satellite and in situ chl estimates decreased with increasing lake size, but the percent difference increased. Related to lake size, the absolute difference between in situ and satellite-derived chl values increased and the percent difference decreased with increasing in situ trophic conditions. Chl values in larger lakes tend to be lower, but large lakes can also be highly heterogeneous (e.g., due to a eutrophic bay with much higher chl than the centre of the lake); this could potentially result in differences between in situ and satellite values depending on the sampling pattern used for the in situ measurements. In terms of the accuracy of satellite chlorophyll as a function of lake productivity, previous work indicates that satellites commonly overestimate chl in lakes with lower chl and underestimate chlorophyll in lakes with higher chl (Stramska et al. 2003, Cota, Wang, and Comiso 2004). Figure 2.5 largely confirmed this prediction, with the satellite estimate more frequently exceeding the in situ estimate for in situ chl values less than $5 \mathrm{mg} \mathrm{m}^{-3}$ and falling below it at high values.

When considering the comparisons between satellite and in situ estimates, however, several allowances regarding the in situ data should be noted. First, all of the in situ chl measurements used in the validation of the method presented here were originally collected by numerous researchers for varying purposes. The exact methods used to measure chl, the number of sampling sites used to generate the estimate and the distribution of those sites across the lake, and the number and timing of sample collections used to generate the estimates all varied among data sources, and for some lakes, some or all of these metadata were not reported. Thus, in some cases, the in situ measurements for a given lake and timespan may not well represent the true mean near-surface chl concentration across the 
whole lake and time period. Non-representative in situ measurements likely contribute significantly to the difference observed between in situ and satellite-derived chl values. The good agreement between in situ and satellite-derived values given this uncertainty, demonstrates the robustness of our approach. However, lacking a global database of standardised lake measurements, we aimed to assemble the best in situ dataset possible given the restrictions imposed by the use of MERIS data and the need to attempt to represent the global diversity of lakes.

Apart from the limitations of the in situ values themselves, the global in situ dataset compiled for this validation over-represents North America and its latitudes and ecoregions, as in situ chl measurements associated with explicit dates and lake location information were much more easily obtained for water bodies in North America than for much of the rest of the world. In particular, we found little suitable field data for South America and Africa, though as noted by Lehner and Döll (2004) and Vernpoorter, Kutser, and Tranvik (2014), the numbers of lakes on these continents greater than $1 \mathrm{~km}^{2}$ are low relative to the mid-northern latitudes, contrasting with the prediction of Downing et al. (2006) based on a model of annual runoff.

\subsubsection{Comparison of chl distributions for MERIS L3 product and new MTRI method}

We compared the distribution of lakewide chl concentrations produced with the new MTRI method, shown in Fig. 2.7, to the distribution of values produced with the MERIS OC4 L3 standard product for the same time periods. Both distributions were normalized by the total number of retrieved lakes. Compared to our global snapshot, the L3 product included a much higher proportion of low-chlorophyll $\left(<5 \mathrm{mg} \mathrm{m}^{-3}\right)$ lakes and lower proportions of higher-chl lakes (Fig. 2.11). This is consistent with our expectation that because the L3 product represents only the largest lakes, it presents a lake distribution skewed towards low chl values. This comparison confirms that the new MTRI method produces a global distribution of lake chl values that is more accurate and representative of all lakes than readily available OC4 global composites. 


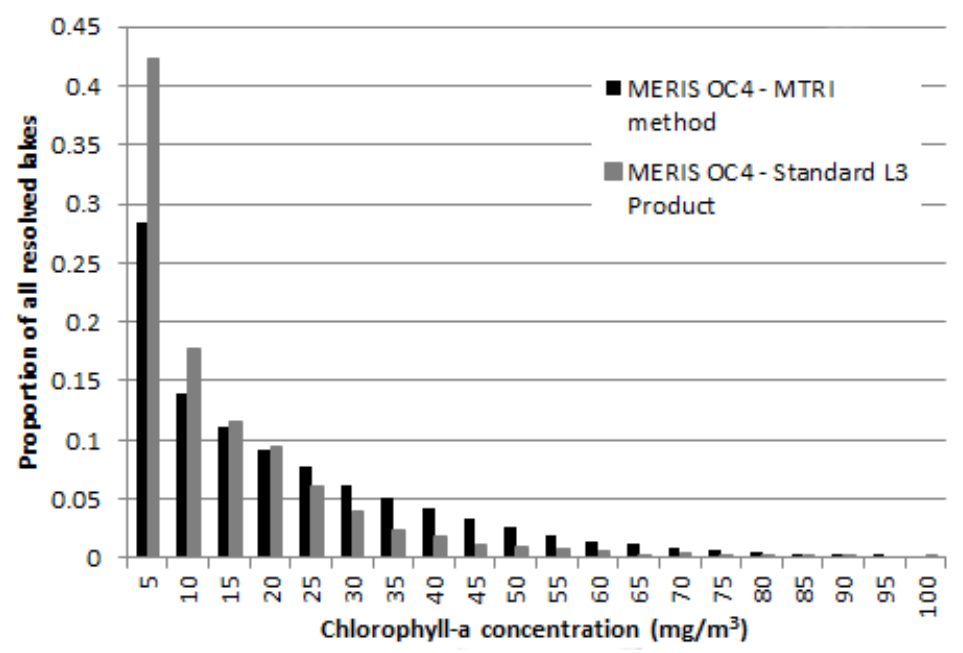

Figure 2.11. Distributions of lake-wide chl values in the MTRI and NASA OceanColor L3 products for global summer 2011 (August for Northern Hemisphere lakes, February for Southern Hemisphere). Both histograms are normalized by the total number of lakes resolved in the respective products.

\subsubsection{Lake detection limitations}

The probability of retrieving chl estimates for a lake was inversely related to lake surface area for our global snapshot. This was expected, as the proportion of lakes presenting a contiguous area of surface water large enough to contain a MERIS pixel decreases with decreasing lake area. Significant proportions of all lake area classes in Fig. 2.12 could not be retrieved by MERIS. These missing lakes include those that cannot be retrieved due to their shape (high shoreline development ratio), particularly impoundments, as well as the lakes for which there were no clear MERIS images in the month used for the global snapshot (August/February 2011 for the Northern and Southern Hemispheres, respectively). 


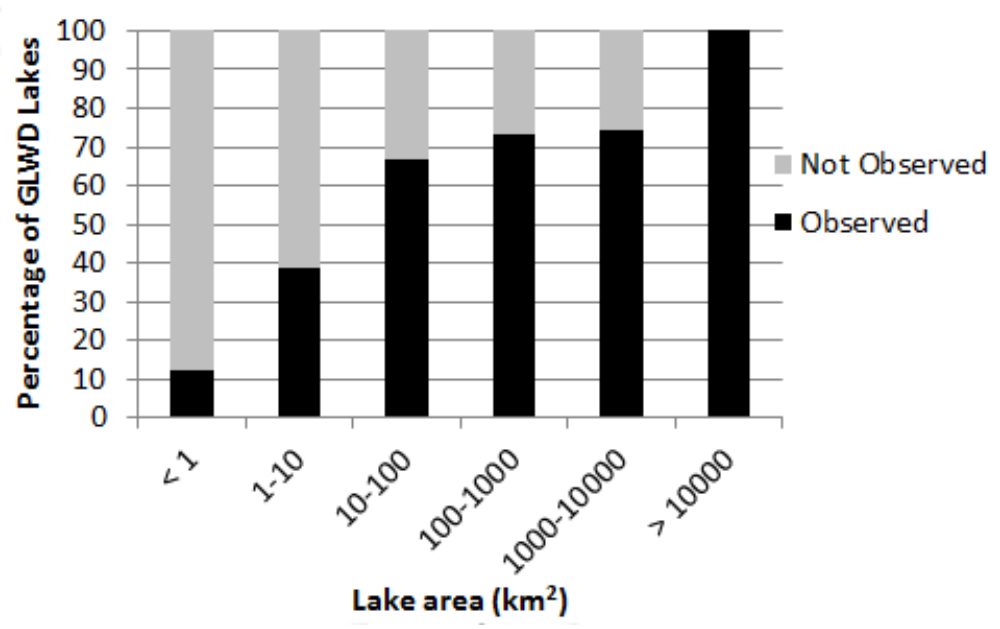

Figure 2.12. Percentage of the lakes included in GLWD Levels 1 and 2 for which chl values could be retrieved from the 2011 growing season global snapshot, separated into several lake surface area size classes.

Because we used the GLWD polygons to identify the locations of lakes, we are not able to estimate the number of lakes that are not included in our results because they are missing from the GLWD. Considering that the GLOWABO database (Vernpoorter, Kutser, and Tranvik 2014) includes roughly twice as many lakes $>1 \mathrm{~km}^{2}$ as the GLWD Levels 1 and 2 data utilized here, the number of lakes that could not be resolved because a better land mask than GLWD 1 and 2 was not available is presumably high. Most of the difference in the number of lakes included in GLWD and GLOWABO was in the $1-10 \mathrm{~km}^{2}$ size range (approx. 150,000 lakes in GLWD vs. 327,000 in GLOWABO). The future availability of GLOWABO or a similar product will therefore greatly increase the number of small lakes for which we can produce chl estimates.

The present work indicates that near-surface chl can be estimated from MERIS imagery for a large portion of the lakes with surface areas $>4 \mathrm{~km}^{2}$ as well as thousands of lakes between $\sim 1$ and $4 \mathrm{~km}^{2}$. Though our global snapshot does not indicate a clear relationship between lake size and chl concentration apart from our observation that very large lakes tended to be less productive, on average (Table 2), it remains possible that the lakes large enough to be resolvable in our dataset are not representative of the lakes $<1 \mathrm{~km}^{2}$ that could 
not be resolved. Lakes $>1 \mathrm{~km}^{2}$ do, however, represent a large fraction of total lake surface area (Verpoorter, Kutser, and Tranvik 2014; Seekell et al. 2013), making the importance of the bias in our snapshot caused by lake size constraints unclear. In the future, availability of a satellite ocean colour sensor with a spatial resolution higher than MERIS would substantially improve the ability to monitor smaller inland lakes, among numerous other benefits.

In addition to inland lakes, our global snapshot also includes chl retrievals for many larger rivers of sufficient width to contain an unmixed water pixel, i.e., rivers of a Strahler order $>5$ (where headwaters are assigned an order of 1 and the stream order increases when streams of the same order intersect; Strahler 1957). Although the total surface area of rivers (360,000 $\mathrm{km}^{2}$ according to Lehner and Döll 2004, which is likely an underestimate) represents a small fraction of the total area of all inland waters relative to lakes, the importance of river fisheries can be disproportionately high in some regions (i.e., Mississippi River in North America, the Amazon River in South America, the Mekong Delta in southeast Asia). As with very small lakes, finer-resolution imagery would be needed to retrieve chl values for most rivers due to their high shoreline development ratio.

Application of the new MTRI method to MERIS imagery throughout an entire year might provide significant insights into intra-annual global chl distribution variability, particularly in the Northern latitudes $\left(>50^{\circ} \mathrm{N}\right)$ where large differences in seasonal water temperature and ice cover duration may be important drivers in annual phytoplankton abundance and productivity. This is especially true when comparing temporally varying high latitude production with more stable equatorial systems. Moreover, monthly global freshwater distributions of chl may provide observations of important episodic bloom events in remote lakes that are not captured in the "global summer" composite nor are regularly sampled in the field. 


\subsubsection{Future potential of Landsat 8}

Medium-resolution ( $\sim 30 \mathrm{~m}$ ) satellite missions (e.g., Landsat) offer the capability to observe lakes much smaller than the minimum mapping unit of traditional ocean colour missions; however, until the recent launch of Landsat 8, these platforms lacked the signal-to-noise ratios (SNR) $(<100)$, spectral channels $(440 \mathrm{~nm})$, resolution ( $100 \mathrm{~nm}$ band widths), and radiometric quantization (8-bit) to produce reliable chl estimates. The new Landsat 8 OLI (Operational Land Imager) has an increased number (11) of narrow wavelength bands (20$50 \mathrm{~nm})$, higher SNR (>200) and 12-bit quantization which support the retrieval of biogeochemical properties, including surface chl concentrations (Pahlevan et al. 2014). Landsat 8 provides the potential to produce reliable and repeatable chl estimates for lakes as small as $0.002 \mathrm{~km}^{2}$ (Verpoorter et al. 2014) which would further improve the estimate of the global freshwater chl distribution. However, there are significant obstacles in generating a Landsat 8 global lake chl distribution including the acquisition of global cloud free imagery given the relatively long temporal revisit (16 days), and massive computational overhead (1 GB file size per scene).

\subsection{Conclusions}

We demonstrated here that ocean colour satellite data can be used to generate reasonably robust estimates of lake-wide chlorophyll for a much larger proportion of freshwater lakes at least $\sim 1 \mathrm{~km}^{2}$ in area than has previously been assumed. Worldwide, the mean estimated lake-wide chlorophyll-a value during the 2011 growing season was $19.2 \pm 19.2 \mathrm{mg} \mathrm{m}^{-3}$ and the median was $13.3 \mathrm{mg} \mathrm{m}^{-3}$. The existing NASA Level 2 and Level 3 OceanColor products for MODIS and MERIS were not suitable for generating a global snapshot of freshwater chl due to the land mask used in Level 2 processing and the reduced resolution of Level 3 products. By processing MERIS OC4 Level 1A data to Level 2 without use of the land/water mask and then compositing that imagery to a global 330-m product, we were able to produce estimates of the growing season chl concentration in 2011 for a much larger number of freshwater lakes. This global dataset will serve as a valuable input for future 
work such as producing global extrapolations of inland fisheries harvest and primary production in inland waters.

\subsection{Acknowledgements}

This work was supported by the U.S. Geological Survey under Grant G12AC20299. Data provided by the European Space Agency. The authors express their appreciation to Scott Hawkins for assistance with data processing. This article is Contribution 1920 of the U.S. Geological Survey Great Lakes Science Center. Any use of trade, product, or firm names is for descriptive purposes only and does not imply endorsement by the U.S. Government. 


\section{Global Carbon Fixation from Freshwater Lakes}

Freshwater phytoplankton productivity, or carbon fixation, is an important water quality parameter that can provide information about the health of lake ecosystems as well as their impacts on regional carbon cycling dynamics. Traditional in-situ methods of measuring and monitoring primary production are unable to capture the necessary spatial and temporal variability at the global scale due to the sheer number and size of the world's lakes. The inability of in situ sampling to quantify production at the global scale is a critical research challenge to better understanding Earth's carbon cycle. Satellite remote sensing offers a potential tool to quantify freshwater lake primary production on a global scale. A new straightforward remote sensing approach was developed to estimate global freshwater carbon fixation from satellite observable lakes. A key component of this approach is the estimation of a light utilization index, $\psi$, for freshwater systems. A significant negative linear model to estimate growing season $\psi$ as a function of latitude was developed from data acquired through an exhaustive literature review. In conjunction with a previous remote sensing generated freshwater chlorophyll abundance data set (Sayers et al. 2015), the $\psi$ model was used to compute growing season carbon fixation for 80,000 freshwater lakes. In general, growing season areal carbon fixation was shown to decrease with increasing latitude in both northern and southern hemispheres. Carbon fixation rates in the southern hemisphere were found to be significantly higher than in the northern hemisphere, with the African continent exhibiting the highest rates. Total daily carbon fixation (areal rate $\mathrm{x}$ lake area) was estimated at $1.03 \mathrm{Tg} \mathrm{C} /$ day with approximately $71 \%$ occurring in the northern hemisphere. Total fixation was highest in North America, which was dominated by the very large number of Canadian shield lakes. In general, total carbon fixation was well explained by total lake surface area, except in the far northern latitudes where lakes are more oligotrophic due to limited nutrient availability. This study resulted in a new freshwater carbon fixation product that provides new insights into the role freshwater lakes play in the global carbon budget. 


\subsection{Introduction}

Freshwater lakes are an important component of the global carbon cycle but they have not always received the attention they deserve (Cole et al. 2007; Tranvik et al. 2009; Williamson et al. 2009; Buffam et al. 2011; Bennington et al. 2012; Lohila et al. 2015). This is largely due to the small fraction of the earth's surface area covered, the large and diverse number of freshwater lakes, and the complex carbon cycle of individual lakes (Cole et al. 2007; Williamson et al. 2009; Buffam et al 2011). Recent work suggests that the carbon cycle of individual lakes can vary on significant temporal and spatial scales depending on thermal stratification, allochthonous loading, trophic state, degree of anthropogenic influence, etc. (Larsen et al. 2011; Moss et al. 2011; Bennington et al. 2012; Regnier et al. 2013). In boreal regions of the world, where many large lakes are located, freshwater lakes play a crucial role in the transformation and storage of carbon (Lohila et al. 2015). The importance of lakes and the complexity of an individual lake's carbon cycle can be illustrated by an example from Lake Superior. Lake Superior, the world's largest lake by surface area, can be a source or sink of $\mathrm{CO}_{2}$ to the atmosphere depending on the time of year, and the variability of this sink/source can vary by two orders of magnitude across the spatial extent of the lake (Bennington et al. 2012).

One of the principal inputs in any lake's carbon budget is the rate of carbon fixation, yet we do not know the carbon fixation for most lakes of the world. This is largely due to the sheer number of freshwater lakes on the planet. It has been estimated that there are over 110 million lakes globally (Verpoorter et al. 2014). Estimating carbon fixation in theses lakes using traditional carbon fixation techniques (e.g., C-14 or oxygen evolution) would be impossible. The more common traditional methods for estimating carbon fixation, C-14 uptake or oxygen evolution, require time consuming bottle incubations which limit observations to lakes in the more accessible and developed regions. Thus, it is likely that estimates of carbon fixation will not exist for the vast majority of lakes on the planet unless new techniques are applied. 
Remote sensing offers the unique ability to synoptically estimate carbon fixation over large areas of the globe. Techniques to estimate carbon fixation over the global ocean using remotely sensed data are not new and have been used successfully to elucidate patterns otherwise undetectable with in situ monitoring. The most well-known model for estimating fixation from satellite observations is that proposed by Behrenfeld and Falkowski (1997) deemed the Vertically Generalized Production Model (VGPM). The VGPM is a mechanistic approach that relies on prediction of the maximum water column photosynthetic rate $\left(\mathrm{P}^{\mathrm{B}}{ }_{\text {opt }}\right)$ rate from water temperature. Behrenfled and Falkowski (1997) used 1000s of co-located observations of water temperature and PBopt to create a $7^{\text {th }}$ order polynomial predictive model. Regionally tuned VGPMs have been used to characterize carbon fixation for many marine regions in the world (Ondrusek et al. 2001, McClain et al. 2002, Marra et al. 2003). Clearly, the VGPM approach relies on extensive and complete measurements in order to achieve the desired degree of accuracy.

Advancements in freshwater remote sensing algorithms have allowed for characterization of phytoplankton abundance and productivity in a variety of different lake environments (e.g., Bergman et al. 2004; Gons et al 2008; Lohrenz et al. 2008; Hunter 2010; Shuchman et al 2013; Fahnenstiel et al. 2016). Remote sensing approaches hold much promise for sampling the large number of lakes on the planet (Sayers et al. 2015) and new studies suggest that now is the time to apply these approaches on a global basis to estimate carbon fixation in freshwater lakes.

Recently, Warner and Lesht (2015) used the oceanic default VGPM parameterization to assess phytoplankton production in the Great Lakes. Significant improvements were made by Fahnenstiel et al. (2016) who developed the Great Lakes Production Model (GLPM) that used remote-sensing (i.e. MODIS) and empirically-derived parameters to accurately estimate lake-wide carbon fixation for the Upper Great Lakes on $1 \mathrm{~km}$ scales. The authors estimated, for the first time, whole lake carbon fixation of the Upper Great Lakes and documented a dramatic recent decrease in phytoplankton abundance and carbon fixation in lakes Huron and Michigan due to the filtering activities of invasive mussels. Similar 
advancements have been made to estimate production in European lakes using remote sensing. Kauer et al. (2015) developed a semi-empirical model to estimate carbon fixation from two small lakes in Estonia using the MERIS sensor. These studies clearly highlight the utility of remote sensing technologies to characterize carbon fixation in freshwater systems.

While these freshwater remote sensing algorithms have provided significant advancements in our understanding of carbon fixation dynamics in several lake environments, they require extensive and detailed in situ calibration data for the application region. In order to assess lake carbon fixation at the global scale a simple methodology is needed that requires limited or no a priori limnological data for model application.

In this study we develop a new approach based on the simple depth integrated model (DIM) suggested by Falkowski (1981) and later Platt (1986) that requires only chlorophyll and irradiance values to estimate, for the first time, carbon fixation for satellite observable lakes of the world. The objectives of this paper are to 1) document the new methodology to estimate carbon fixation from freshwater lakes with limited data sets, and 2) to generate a new global estimate of "growing season" carbon fixation from satellite observable freshwater lakes.

\subsection{Methods}

\subsubsection{Model Development}

Because the remote sensing derived products for the 80,000 satellite observable lakes of the world were limited to chlorophyll and irradiance, it is infeasible to generate carbon fixation estimates without the other necessary higher order model input parameters (i.e. maximum photosynthetic rates) required from more advanced production models (e.g. Behrenfeld and Falkowski 1997). Therefore, a straightforward methodology was developed to calculate carbon fixation for the world's lakes that requires limited input

parameters. The simplified depth integrated model (DIM) approach suggested by 
Falkowski (1981) and later by Platt (1986) meets these criteria, and with proper calibration, can provide accurate estimates of primary production for freshwater lakes. The methodology of Falkowski (1981) and Platt (1986) uses the following three inputs: chlorophyll concentration, PAR, and the light-utilization index (characterizes phytoplankton photosynthetic rate) to derive water column carbon fixation.

$$
\mathrm{P}=\psi \times \mathrm{C} \times \mathrm{E}_{\mathrm{o}} \quad \mathrm{EQ} 1
$$

where $\mathrm{P}$ is daily water column carbon fixation, $\psi=$ light utilization index, $\mathrm{C}=$ integrated chlorophyll concentration over the euphotic zone and $\mathrm{E}_{\mathrm{o}}=$ daily photosynthetically active (PAR) irradiance.

The light utilization index describes the rate of photosynthesis per unit chlorophyll at a specific irradiance level following:

$$
\frac{P}{C}=E_{0} * \psi \quad \mathrm{EQ} 2
$$

Using coincident observations of $\mathrm{P}, \mathrm{C}$, and irradiance, EQ2 can be rearranged to solve for $\psi$.

The light-utilization index is relatively well characterized for a variety of marine environments, however, it has not been evaluated for freshwater systems. In order to characterize $\psi$ for freshwater systems a robust set of calibration data was required. Coincident observations of production and chlorophyll were compiled from discrete measurements made in the Laurentian Great Lakes reported by Fahnenstiel et al. (2016) as well as from values reported in the literature from other freshwater systems. The Great Lakes depth integrated production values were reported in units of $\mathrm{mg} \mathrm{C} / \mathrm{m} 2 /$ day and surface chlorophyll concentrations in $\mathrm{mg} / \mathrm{m} 3$. Coincident measurements of the light extinction coefficient were used to derive the euphotic zone depth. Surface chlorophyll values were multiplied by the calculated euphotic depth to estimate water column integrated chlorophyll biomass, $\mathrm{C}$ (mg) which assumes a uniformly mixed water column. Photosynthetically active radiation (PAR) irradiance was derived using the methods 
described in Fahnenstiel et al. (2016) which used The NOAA National Centers for Environmental Prediction (NCEP) Climate Forecasting System version 2 (CFSv2) (Saha et al. 2014) incoming shortwave radiation product to model PAR. Briefly, shortwave radiation flux was converted to PAR flux (W/m2) (McCree, 1981) and then to photon flux $(\mathrm{E} / \mathrm{m} 2 / \mathrm{s})$. Great Lakes $\psi$ values were then computed from matchups of $\mathrm{P}, \mathrm{C}$, and Eo following EQ2.

Additional $\psi$ values were derived from coincident measurements of primary production and chlorophyll extracted from an exhaustive literature review. Papers were identified through exhaustive searches using keyword terms associated with freshwater primary production, photosynthesis, fixation rate, assimilation number etc. Identified papers were screened to determine if usable data was present (e.g. data from growing season). Data (production and chlorophyll) was extracted from tables when present. In the case where data was presented on plots, values were retrieved using a gridded ruler to correspond data points to a tick on the plot axes. In the case of dense plots, data points were extracted from only those points that are clearly identifiable (i.e. peaks and troughs).

In the cases where chlorophyll was reported as a single rate value (e.g. $\mathrm{mg} / \mathrm{m}^{3}$ ) additional data was needed to estimate photic zone or mixed layer depth for a given sample. Diffuse attenuation coefficients for PAR (Kpar) and secchi disc depths were often reported. In the case of Kpar data, euphotic zone depth was computed as 4.605/kpar (Lee et al. 2007). Secchi disc measurements were converted to Kpar following the method reported in Fahnenstiel et al. (2016).

Irradiance was estimated following methods from Fahenstiel et al (2016) for each retrieved data point using the Climate Forecasting System Reanalysis (CFSR) for data from 19792011, and the Climate Forecasting System Version 2 (CFSv2) for data from 2011 to present. For data observations the were reported over a defined time period irradiance values were acquired over the same period and the mean value was computed and used as the irradiance value. 
In total 52 studies from the 1960s to present were used to produce data sets for characterization of $\psi$ (Table 3.1). The combined observations from the Great Lakes and literature totaled 46 lakes. Mean $\psi$ values were computed for each lake reported in each study or the Great Lakes which resulted in 52 unique values of $\psi$. The observations spanned the latitude range from $<1$ degree to 74 degrees where approximately $80 \%$ of the observations were from latitudes between 35-52 degrees. Because of the uneven data distribution, observations were averaged into 3 degree bins. The binning resulted in 14 data observations.

Table 3.1. Data used to generate the latitude vs. light utilization index model. The reference for each lake study is shown on the table.

\begin{tabular}{ll}
\hline \multicolumn{1}{c}{ Lake Name } & \multicolumn{1}{c}{ In Situ Source } \\
\hline Lake Huron & Fahnenstiel Unpublished \\
Lake Michigan & Fahnenstiel Unpublished \\
Lake Superior & Fahnenstiel Unpublished \\
Lake Tangayika & Hecky et al. 1978, 1981 \\
Kinneret Lake & Berman et al. 1995 \\
Lake Tangayika & Stenuite et al. 2007 \\
Lake Ontario & Millard et al. 1996 \\
Lake Malawi & Guildford et al. 2007 \\
Char Lake & Klaff and Welch 1974 \\
GTH 112 & Whalen et al. 2006 \\
GTH 114 & Whalen et al. 2006 \\
Lake Chad & Lemoalle 1981 \\
Tissawewa & Amarasinghe and Vijverberg2002 \\
Parakrama Samudra & Dokulil et al. 1983 \\
Green Lake & Fee et al. 1992 \\
Orange Lake & Fee et al. 1992 \\
Linge Lake & Fee et al. 1992 \\
Musclow Lake & Fee et al. 1992 \\
Sydney Lake & Fee et al. 1992 \\
Trout Lake & Fee et al. 1992 \\
Lake Nipigon & Fee et al. 1992 \\
Lake Superior & Fee et al. 1992 \\
Naivasha Lake & Melack 1979 \\
Oloidien Lake & Melack 1979 \\
Winam Gulf & Melack 1979 \\
\end{tabular}


Lake Malawi

Lake Superior

Lake Huron

Lake Erie

Canyon Ferry Lake

Lake Minnetonka

Gaynor Lake

Haydens Lake

Allens Lake

Pass Lake

Black Lake

Lake Ontario

Lake Michigan

Washington Lake

Bluehill Lake

Clear Lake

Sammamish Lake

John H. Kerr Reservoir

Severson Lake

Tuttle Creek Reservoir

Shagawa Lake

Lake Tahoe

Loch Leven

Vombsjob Lake

Joseph Lake

Rosseau, Skeleton Lakes

Muskoka, Dudley, Gravenhurst Lakes
Patterson et al. 2000

Dobson et al. 1974

Vollenweider et al. 1974

Vollenweider et al. 1974

Wright 1959

Megard 1972, 1974

Winner 1972

Winner 1972

Winner 1972

Winner 1972

Winner 1972

Vollenweider et al. 1974

Rousar 1973

Edmondson 1979

Kereskes 1974, 1975

Schindler and Comita 1972

Welch et al. 1977

Weiss and Moore 1977

Schindler and Comita 1972

Marzolf and Osborne 1972

Megard 1972, 1974

Goldman 1977

Bindloss 1974

Gelin 1971

Michalski et al. 1973

Michalski et al. 1973

Michalski et al. 1973

\subsubsection{Model Validation}

To evaluate the new DIM production estimates we compared the model results to in situ production values reported in the literature for several lakes throughout the globe. Independent validation values were acquired from multiple studies following the same methods reported above. The validation data sets were reported as monthly mean values from the Laurentian Great Lakes and several African Lakes that ranged from 2002 to 2009. This resulted in 8 eight unique observations to be used for DIM model validation (Table 
3.2). DIM model input paramters (chlorophyll and irradiance) were derived for the time periods represetnted by each in situ data value. The DIM was applied using those input parameters to generate modeled carbon fixation for acomparison with the in situ data points.

Table 3.2. Independent data used to validate the DIM model. The mean values for in situ observations and DIM retrievals are shown on the table.

\begin{tabular}{llccl}
\hline \multicolumn{1}{c}{ Lake Name } & \multicolumn{1}{c}{ Time Period } & In situ & DIM & \multicolumn{1}{c}{ In Situ Source } \\
\hline Lake Michigan & March-Nov, 2009 & 273 & 171 & Fahnenstiel Unpublished \\
Lake Erie & June-Sept, 2002 & 1266 & 723 & Porta et al. 2005 \\
Lake Erie & June-Oct, 2003 & 249 & 664 & Porta et al. 2005 \\
Kivu & June-Oct, 2002 & 461 & 928 & Darchambeau et al. 2014 \\
Kivu & Jan-June, 2003 & 784 & 931 & Darchambeau et al. 2014 \\
Ziway & Jan-Dec, 2005 & 1060 & 1732 & Tilahun and Algren 2010 \\
Awasaa & Jan-Dec, 2006 & 3160 & 1309 & Tilahun and Algren 2010 \\
Chamo & Jan-Dec, 2007 & 1590 & 1681 & Tilahun and Algren 2010 \\
& Mean & $\mathbf{1 1 0 5}$ & $\mathbf{1 0 1 7}$ & \\
\hline
\end{tabular}

Mean DIM model input parameters (i.e. Chl and Irradiance) were computed from satellite data (MERIS) corresponding to the time periods represented for each in situ sample (e.g. monthly or seasonal mean values). Unless a defined location (i.e. coordinates) for a sample was provided, the lake-wide mean satellite retrieved values were used to compare with in situ values. If the exact location was provided, mean values were computed using a 5-by5 window surrounding the sample point.

\subsubsection{Remote Sensing Model Application}

Chlorophyll-a estimates for $\sim 80,000$ lakes were estimated for the global summer (August in northern hemisphere, February in southern hemisphere) for 2011 using the MERIS satellite sensor reported in Sayers et al. 2015. Briefly, every swath of Level 1 MERIS data was acquired from the NASA Ocean Biology Processing Group (OBPG) for the whole globe over the month of August (northern hemisphere) and February (southern 
hemisphere). All images were processed to Level 2 using $12 \mathrm{gen}$ with custom defined land/water masks. Chlorophyll-a concentration (OC4E, O'Reilly et al. 1998) was then calculated for every valid water pixel in every swath excluding the global ocean. Mean chlorophyll-a was then computed for every pixel over the month long period for each hemisphere. Finally, the mean chlorophyll-a dataset was intersected with the GLWD lake boundary layer and descriptive statistics were derived for each lake with valid chlorophylla pixels.

Hourly PAR irradiance data was derived for each day in the global summer month (August, February) for the whole globe on a $30 \mathrm{~km}$ x $30 \mathrm{~km}$ resolution grid. Lakes completely inscribed in an irradiance grid cell used the irradiance from that cell, while lakes spanning multiple cells used the mean value from the multiple cells.

The spectral diffuse attenuation coefficient (Kd) was calculated using the semi-analytical approach suggested by Lee et al. (2005), which requires wavelength dependent absorption and backscatter coefficients in addition to solar zenith angle as input parameters. Solar zenith angles were derived using Julian date and latitude for each lake where latitude is calculated as the geometric center of each lake polygon. The absorption and backscatter coefficients were computed as the sum of pure freshwater absorption $\left(\mathrm{a}_{\mathrm{w}}\right)$ and backscatter $\left(b_{b w}\right)$ and phytoplankton absorption $\left(a_{\mathrm{ph}}\right)$ and backscatter $\left(b_{\mathrm{bphy}}\right)$. Pure water absorption is assumed (Pope and Fry 1997). Note, absorption due to CDOM and non-algal particles (NAP) are not considered here as there were no remote sensing products available for all $\sim 80,000$ lakes in which to estimate their contribution to total absorption, therefore our estimate of light attenuation will be lower (deeper photic depth) than reality in some lakes with large CDOM and NAP components.

Chlorophyll-a is the dominant absorbing pigment for most phytoplankton, therefore the spectral phytoplankton absorption coefficient can be derived from the product of chlorophyll-a concentration and the mass specific phytoplankton absorption coefficient $\left(a_{\mathrm{ph}}{ }^{*}\right)$. The spectral shape and magnitude of $\mathrm{a}_{\mathrm{ph}}{ }^{*}$ can vary due to pigment packaging where larger individual phytoplankton cells exhibit a greater degree of packaging. The Bricaud et 
al. (2005) model estimates spectral $\mathrm{a}_{\mathrm{ph}}{ }^{*}$ values from chlorophyll concentration. This model was used to estimate $\mathrm{a}_{\mathrm{ph}}$ * from the remote sensing derived Chl-a values for each lake. Finally, the phytoplankton absorption was calculated as the product of the estimated $\mathrm{a}_{\mathrm{ph}}$ * and the observed Chl-a for each lake.

Light scattered from phytoplankton particles also play an important role in how radiant energy is reflected out of the water to be observed by remote sensing platforms. The particulate backscatter $\left(b_{\mathrm{bp}}\right)$ coefficient is assumed to be expressly due to phytoplankton scattering, thus $b_{b p}$ was derived from the Chl-a values using published values of the mass specific phytoplankton scattering coefficient $\left(\mathrm{b}_{\text {phy }}{ }^{*}\right.$ ) (Gilerson et al.2007) and the backscattering ratio (Binding et al. 2012). Chl-a was multiplied by $b_{\text {phy }} *$ to calculate the total scattering coefficient which was then multiplied by the backscatter ratio to estimate the total scattered light from phytoplankton in the backward direction.

The diffuse attenuation coefficient for PAR (KPAR) was estimated using the methods described in Fahnenstiel et al. (2016) which used an empirical relationship (Saulquin et al. 2013) to convert retrieved KD490 to KPAR. The photic zone depth (m) was calculated as 4.605/KPAR (Bukata et al. 1995).

The water column integrated chlorophyll-a biomass used in the DIM was computed as the product of the satellite derived surface concentration $\left(\mathrm{mg} / \mathrm{m}^{3}\right)$ and the estimated photic zone depth (m) to produce a total water column chlorophyll-a biomass (mg). This approach assumes a vertically mixed water column with constant chlorophyll at each depth layer in the photic zone.

Global freshwater carbon fixation was calculated for the global summer period using the satellite derived chlorophyll, KD, and irradiance data sets described above. Carbon fixation was computed each day within the month representing the global summer (August in northern hemisphere, February in southern hemisphere) using the hourly irradiance data and mean chl and $\mathrm{kd}$ data. The global summer carbon fixation rates $\left(\mathrm{mg} \mathrm{C} / \mathrm{m}^{2} /\right.$ day) for each lake were calculated as the mean of the daily rate values. Mean daily total carbon fixation 
(Tg C) was computed for each lake as the mean summer fixation rate multiplied by the lake area (Tg C/day).

Total and areal carbon fixation for the 80,000 lakes were aggregated into the Freshwater Ecoregions of the World (FEOW) regionalization scheme. There are 426 unique units in the finest resolution product that generally correspond to watersheds or primary drainage basins of large lakes or dense groupings of lakes with similar assemblages of species and natural communities. Descriptive statistics including mean, standard deviation, and range for carbon fixation values were computed for each ecoregion unit from lakes inside the unit. Additional information was recorded for each ecoregion unit including the mean and total area the number of lakes as well as.

\subsection{Results}

\subsubsection{LUI Model}

In order to calculate carbon fixation for global freshwater lakes using the DIM approach, (Falkowski 1981, Platt 1986) a straightforward model was required to estimate the light utilization index $(\psi)$. Freshwater growing season $\psi$ values ranged from 0.20 to 1.17 with mean value of $0.49 \mathrm{~g} \mathrm{C}(\mathrm{g} \mathrm{Chl})^{-1} \mathrm{~m}^{2}(\mathrm{E})^{-1}$. A significant decreasing linear relationship between $\psi$ and latitude was established for global growing season in both the northern and

southern hemispheres ( $\mathrm{p}$-value $=0.001, \mathrm{R}^{2}=0.57$ ) (Fig 1). This model was used to compute global freshwater carbon fixation for the global growing season. 


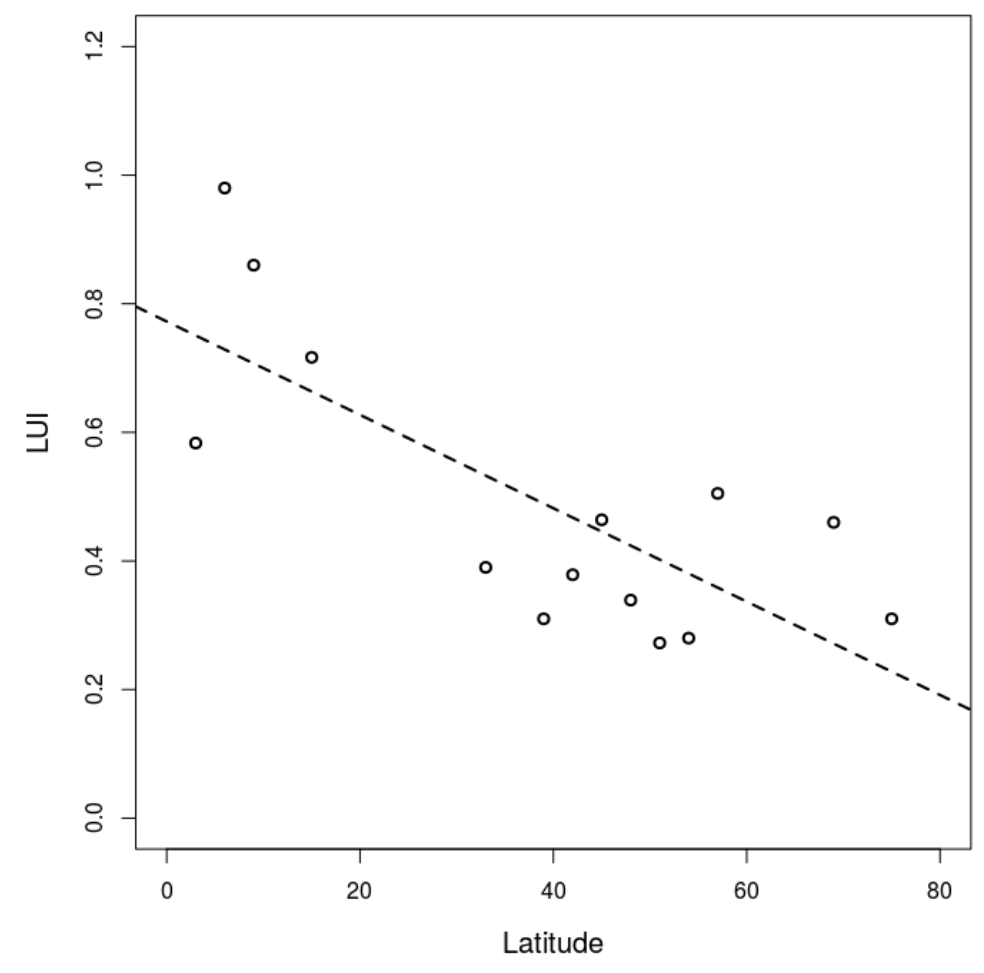

Figure 3.1. Relationship of light utilization index $(\psi)$ and latitude for global freshwater lakes during the growing season. Because approximately $80 \%$ of our observations were from latitudes 35-52 degrees, we bin averagedthe latitude data (3 degrees). The decreasing linear relationship between latitude and LUI was significant ( $\mathrm{p}$-value $=0.001, \mathrm{R}^{2}=0.57$ ).

\subsubsection{LUI Validation}

Carbon fixation results for the global growing season using remote sensing data inputs (Chlorophyll, KD, irradiance) and the empirical LUI model agreed well with measured carbon fixation rates. Mean satellite LUI retrieved carbon fixation values $(1017 \mathrm{mg}$ $\mathrm{C} / \mathrm{m} 2 /$ day) were within approximately $8 \%$ of corresponding in situ carbon fixation values (1105 mg C/m2/day) for North American and African Lakes. These results suggest the simple DIM model with empirical LUI parameterization from latitude can be used to produce robust growing season carbon fixation estimates in global freshwater lakes. 


\subsubsection{Areal Carbon Fixation}

Using the simple DIM model, mean areal carbon fixation in $~ 80,000$ lakes for the 2011 growing season was estimated. Spatial aggregation (mean values) of the $\sim 80000$ lakes into freshwater ecoregions of the world (FEOW) (Abell et al. 2008) provides for a generalized synoptic global assessment of freshwater carbon fixation. For most of the FEOWs, the model was able to estimate carbon fixation in freshwater lakes (Fig 3.2). Note, in Figure 3.2 ecoregions where no lakes were observed are colored gray.

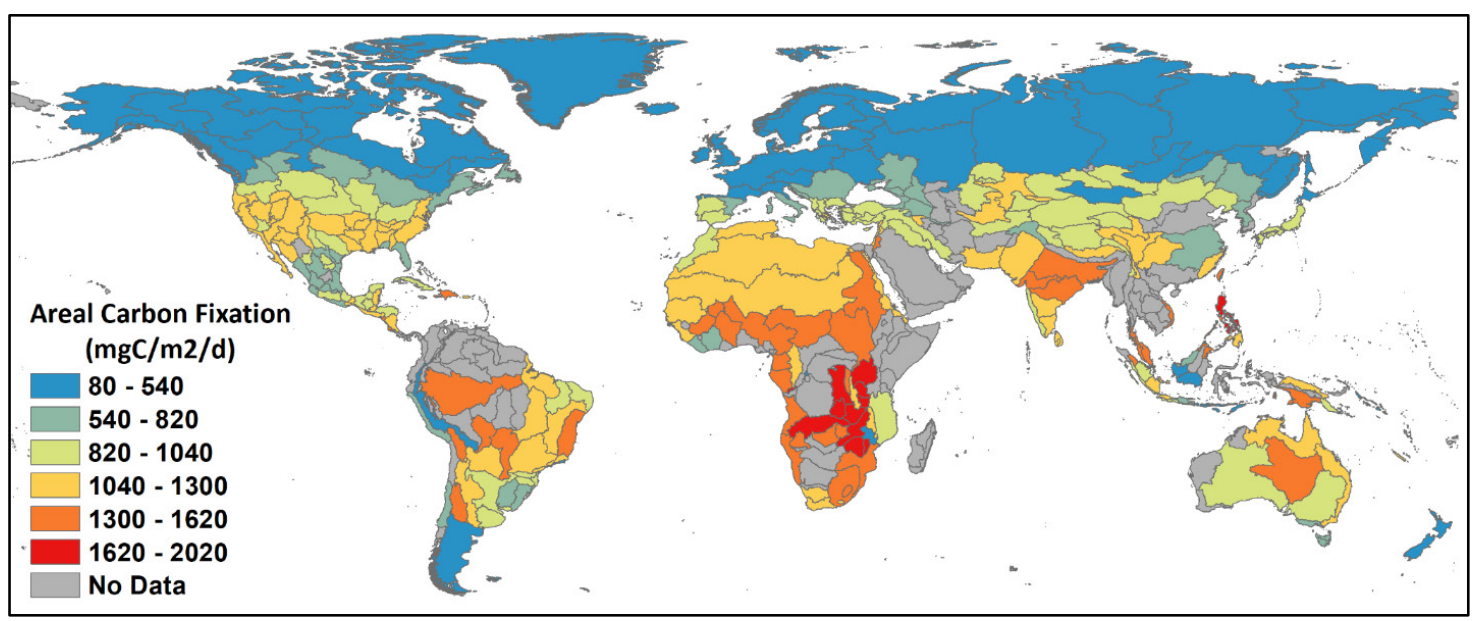

Figure 3.2. Mean growing season areal carbon fixation $\left(\mathrm{mg} \mathrm{C} / \mathrm{m}^{2} / \mathrm{d}\right)$ in freshwater lakes aggregated by freshwater ecoregions of the world (Abell et al. 2008).

In general, growing season carbon fixation decreases with increasing latitude for both the northern and southern hemispheres. In the southern hemisphere less than $<15 \%$ of the freshwater ecoregions had satellite-resolvable lakes whereas in the northern hemisphere $\sim 75 \%$ of the ecoregions had satellite-resolvable lakes. Statistically significant regressions were found for carbon fixation and latitude for both hemispheres and there was no significant difference between regression models for northern and southern hemispheres (Fig 3 left panel: northern hemisphere, $\mathrm{y}=-13.5+1279, \mathrm{R}^{2}=0.45, \mathrm{p}<0.001, \mathrm{n}=217$; right panel: southern hemisphere, $\mathrm{y}=-14.8+1227, \mathrm{R}^{2}=0.26, \mathrm{p}<0.05, \mathrm{n}=16$ ). In the northern hemisphere, latitude alone explained almost $50 \%$ of the variation in carbon fixation. 
Southern hemisphere observations by latitude were more variable than for the northern hemisphere observations, however, fewer observations in the southern hemisphere limits interpretation using FEOW.

Areal carbon fixation also varied significantly by continent (ANOVA p-value $<0.001$ ) (Fig 3.4). Mean fixation rates were significantly different for all continents with the exception of South America and Australia. Africa exhibited the highest mean fixation rates which were $\sim 1.5$ times greater than the next highest (South America) and more than four-fold larger than North America and Europe.
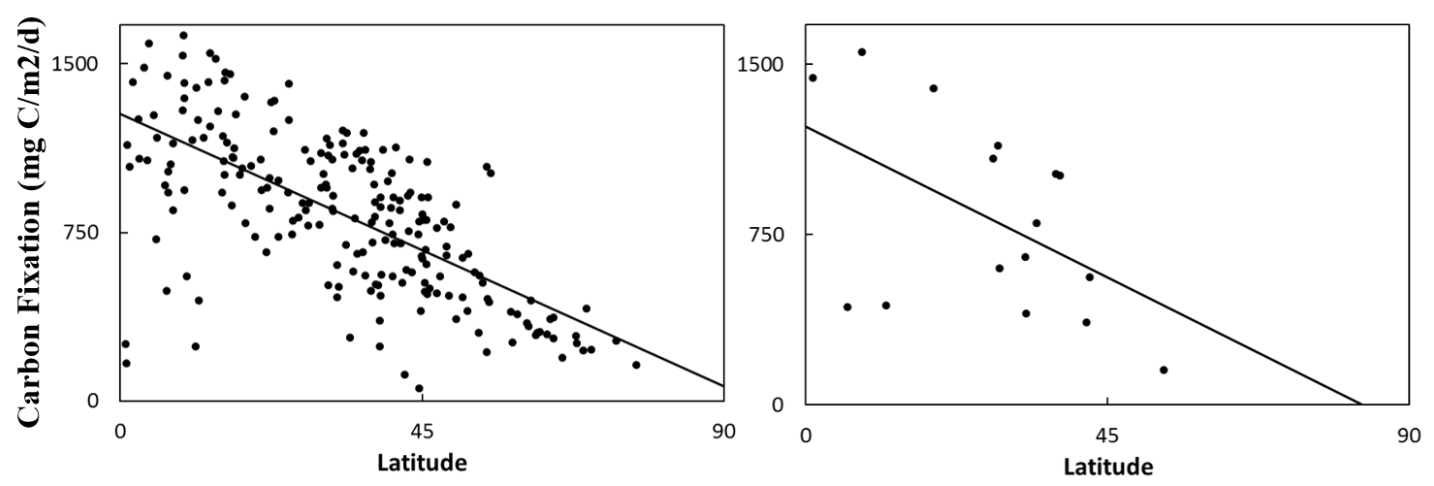

Figure 3.3. Areal carbon fixation $\left(\mathrm{mg} \mathrm{C} / \mathrm{m}^{2} / \mathrm{d}\right)$ aggregated by freshwater ecoregions of the world vs. latitude for northern $(y=-13.479 x+1279.3$, $\mathrm{R}^{2}=0.45, \mathrm{p}<0.001, \mathrm{n}=217$ and southern hemispheres $(\mathrm{y}=-14.781 \mathrm{x}+1227.1$, $\mathrm{R}^{2}=0.26, \mathrm{p}=0.046, \mathrm{n}=16$ ). 


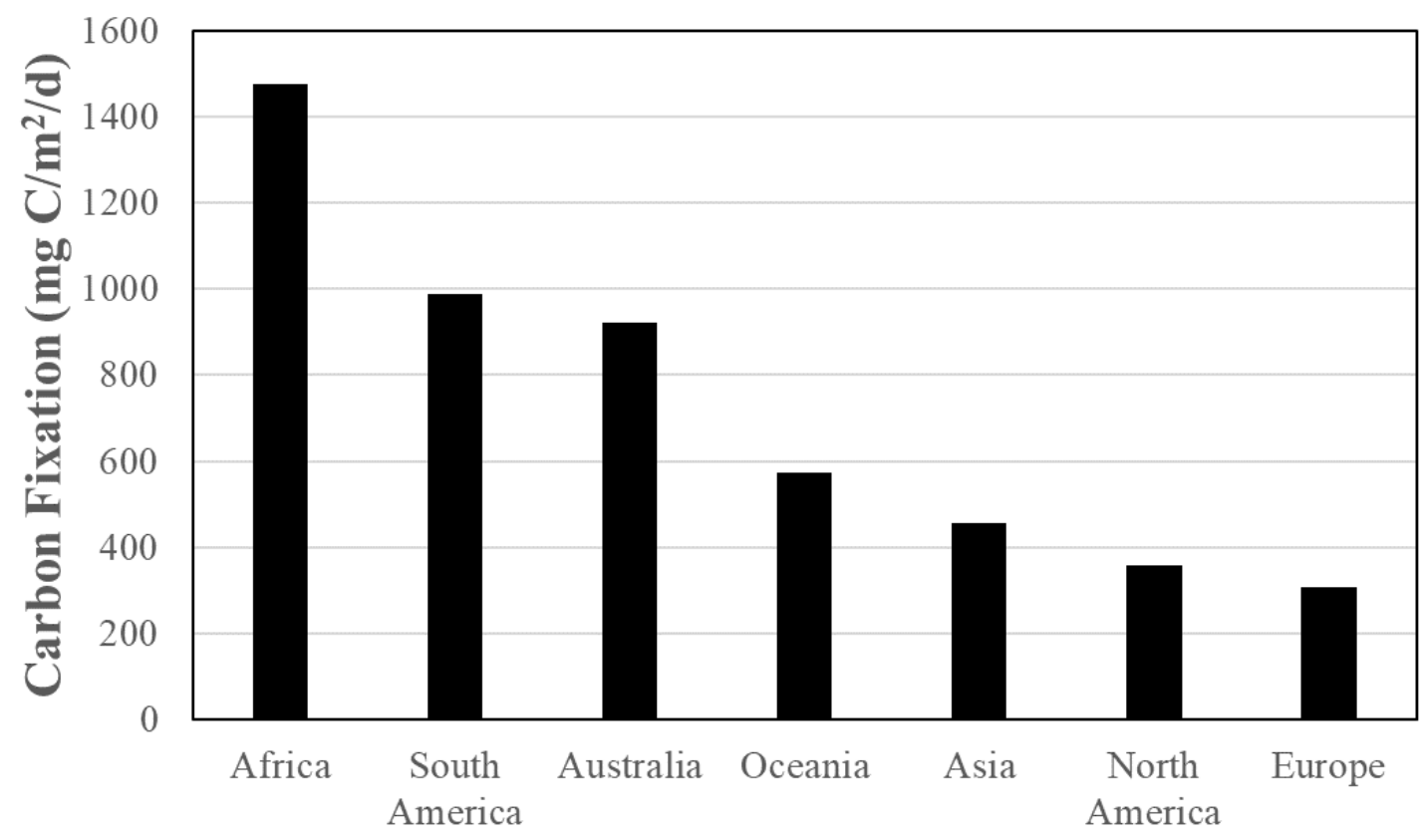

Continent

Figure 3.4. Mean continental growing season areal carbon fixation (mg $\left.\mathrm{C} / \mathrm{m}^{2} / \mathrm{d}\right)$.

When considering all $\sim 80,000$ lakes individually, the southern hemisphere generally exhibited carbon fixation rates higher than the northern hemisphere (northern hemisphere: mean $=365$, median $=300$; southern hemisphere: mean $=1101$, median $=1116$ ). This is partially explained by the fact that southern hemisphere lakes are generally located closer to the equator (thus higher LUI values) than the northern hemisphere lakes (southern hemisphere lake latitude: mean $=21.9$, median 22.5 degrees $\mathrm{S}$; northern hemisphere lake latitude: mean $=60.3$, median $=62.4$ degrees $\mathrm{N}$ ). However, comparing the mean fixation rates for the southern and northern hemispheres for common latitude ranges (i.e. 0-30 and 30-60 degrees, no southern lakes further than $60 \mathrm{~S}$ ) suggested the southern hemisphere exhibits generally higher rates of carbon fixation than the northern hemisphere at similar latitudes. The southern hemisphere mean carbon fixation from 0 to 30 degrees was significantly higher than the northern hemisphere mean fixation rate from 0 to 30 degrees (southern 0-30: mean $=1297$; northern 0-30: mean $=968$; p-value $<0.005)$. Similarly, the southern hemisphere mean fixation rate for 30 to 60 degrees was significantly greater than 
the northern hemisphere fixation rate for the same latitude range (southern 30-60: mean $=$ 728; northern 30-60: mean $=498$; p-value $<0.005$ ).

Mean chlorophyll concentrations were different between northern and southern hemispheres (northern $=17.6 \mathrm{mg} / \mathrm{m}^{3}$; southern $=19.2 \mathrm{mg} / \mathrm{m}^{3}$ ). The biggest differences between hemispheres was for the 0-30 degree latitude range where the northern hemisphere had mean value of $11.6 \mathrm{mg} / \mathrm{m}^{3}$ while the southern hemisphere was $17.1 \mathrm{mg} / \mathrm{m}^{3}$. Mean values were similar between the hemispheres for the 30-60 degree latitude range (northern $=18.4 \mathrm{mg} / \mathrm{m}^{3}$; southern $=18.5 \mathrm{mg} / \mathrm{m}^{3}$ ).

There was a weak log-log relationship identified between growing season areal carbon fixation rate and lake size for either northern or southern hemispheres (northern hemisphere $y=0.1647 x+5.448, p$-value $<0.001, R^{2}=0.06$; southern hemisphere $y=0.0658 x+6.6793$, $\mathrm{p}$-value $\left.<0.001, \mathrm{R}^{2}=0.03\right)$. Figure 3.5 is a log-log plot depicting the relationship between lake area and areal carbon fixation for the northern hemisphere (top panel) and southern hemisphere (bottom panel). Even though there is no significant relationship between lake size and fixation rate, general patterns can be perceived. From the plot it can be observed that for both hemispheres lakes larger than $100 \mathrm{~km}^{2}$ generally exhibited smaller ranges $(\sim 1$ order of magnitude) of areal carbon fixation than did lakes smaller than $100 \mathrm{~km}^{2}(\sim 2$ orders of magnitude). Similarly, low carbon fixation rates $(<100 \mathrm{mg} \mathrm{C} / \mathrm{m} 2 / \mathrm{d})$ are generally observed in lakes smaller than $100 \mathrm{~km}^{2}$ in both hemispheres. 

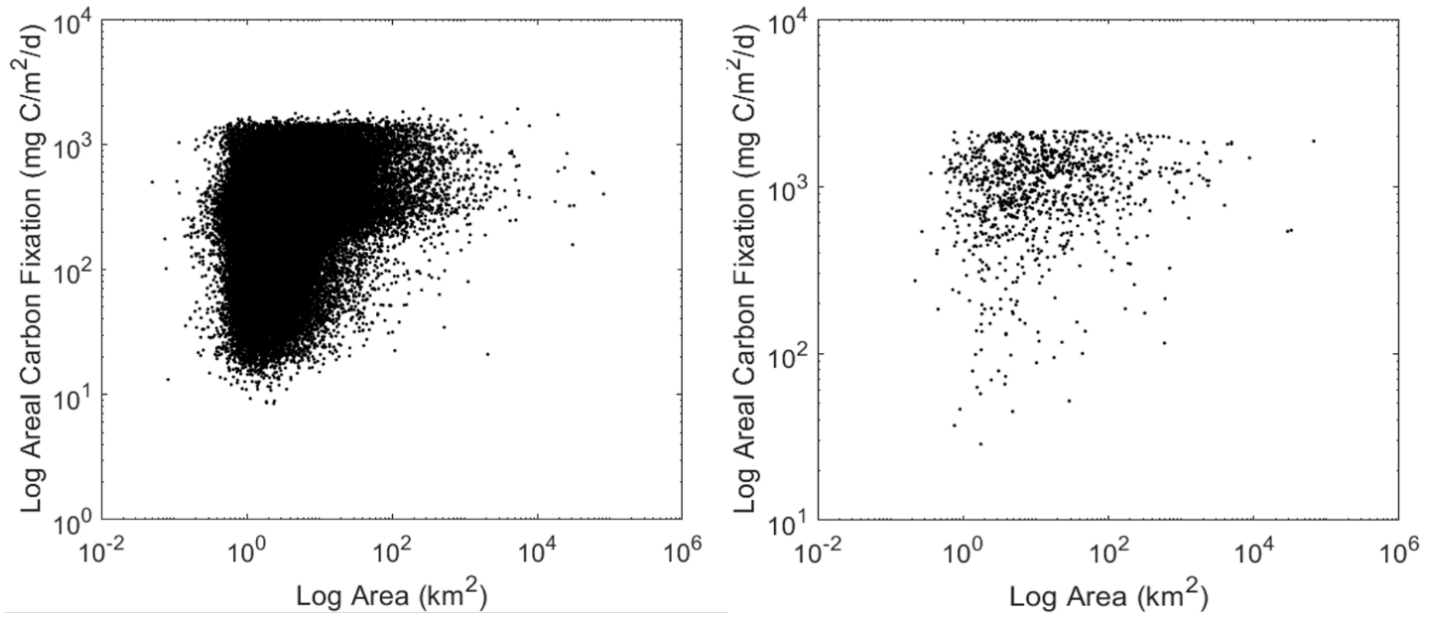

Figure 3.5. Log-log plots of individual lake area and areal carbon fixation for all 80000 lakes grouped by hemisphere (northern - left panel, southern - right panel).

Areal carbon fixation rates aggregated by lake size were compared between northern and southern hemispheres. For each hemisphere, lakes were grouped into size ranges of 0-10, $10-100,100-1000,>1000 \mathrm{~km}^{2}$. Southern hemisphere mean areal fixation rates were significantly greater than the northern hemisphere mean rates for all lake size groups (010: $\mathrm{N}=338, \mathrm{~S}=1019$, p-value $<0.005 ; 10-100: \mathrm{N}=509, \mathrm{~S}=1179$, $\mathrm{p}$-value $<0.005 ; 100$ 1000: $\mathrm{N}=630, \mathrm{~S}=1194$, $\mathrm{p}$-value $<0.005 ;>1000: \mathrm{N}=594, \mathrm{~S}=1279$, $\mathrm{p}$-value $<0.005)$. The observed differences in fixation rate by lake size between hemispheres is partially explained by the large number of lakes at far northern latitudes (e.g. $>60 \mathrm{~N})$ and no lakes in the southern hemisphere at the same latitude range. However, comparing mean fixation rates by size groups in the latitude range where lakes are present in both hemispheres (060 degrees) yields similar results to the full latitude comparison. For the common latitude range, southern hemisphere mean areal fixation rates were significantly greater than the northern hemisphere mean rates for all lake size groups $(0-10: \mathrm{N}=480, \mathrm{~S}=1019$, $\mathrm{p}$-value $<0.005 ; 10-100: \mathrm{N}=665, \mathrm{~S}=1179$, p-value $<0.005 ; 100-1000: \mathrm{N}=779, \mathrm{~S}=1194$, p-value $<0.005$; $>1000: \mathrm{N}=695, \mathrm{~S}=1279$, p-value $<0.005)$. These results strongly suggest that 
lakes in the southern hemisphere are more productive than similarly sized lakes in the northern hemisphere.

\subsubsection{Total Carbon Fixation}

The DIM approach was also used to estimate the total daily freshwater lake carbon fixation (Tg $\mathrm{C} /$ day) for the global growing season. The total freshwater carbon fixation was estimated as $1.03 \mathrm{Tg} \mathrm{C} /$ day or $376 \mathrm{Tg} \mathrm{C} /$ year. The northern hemisphere accounted for $\sim 71$ $\%$ ( $0.74 \mathrm{Tg} \mathrm{C} /$ day) of the global total while the southern hemisphere accounted for $\sim 29 \%$ $(0.30 \mathrm{Tg} \mathrm{C} /$ day). The northern hemisphere total fixation was produced from 78927 lakes (98.6\% of all lakes) while the southern hemisphere fixation was produced from just 1074 lakes ( $1.4 \%$ of all lakes). Total freshwater carbon fixation by continent is shown in Figure 3.6. North America has the highest amount of total fixation $(0.52 \mathrm{Tg} \mathrm{C} / \mathrm{d})$ and is almost two-fold larger than Africa (0.28 Tg C/d). Together North America and Africa account for almost $80 \%$ of the global total carbon fixation.

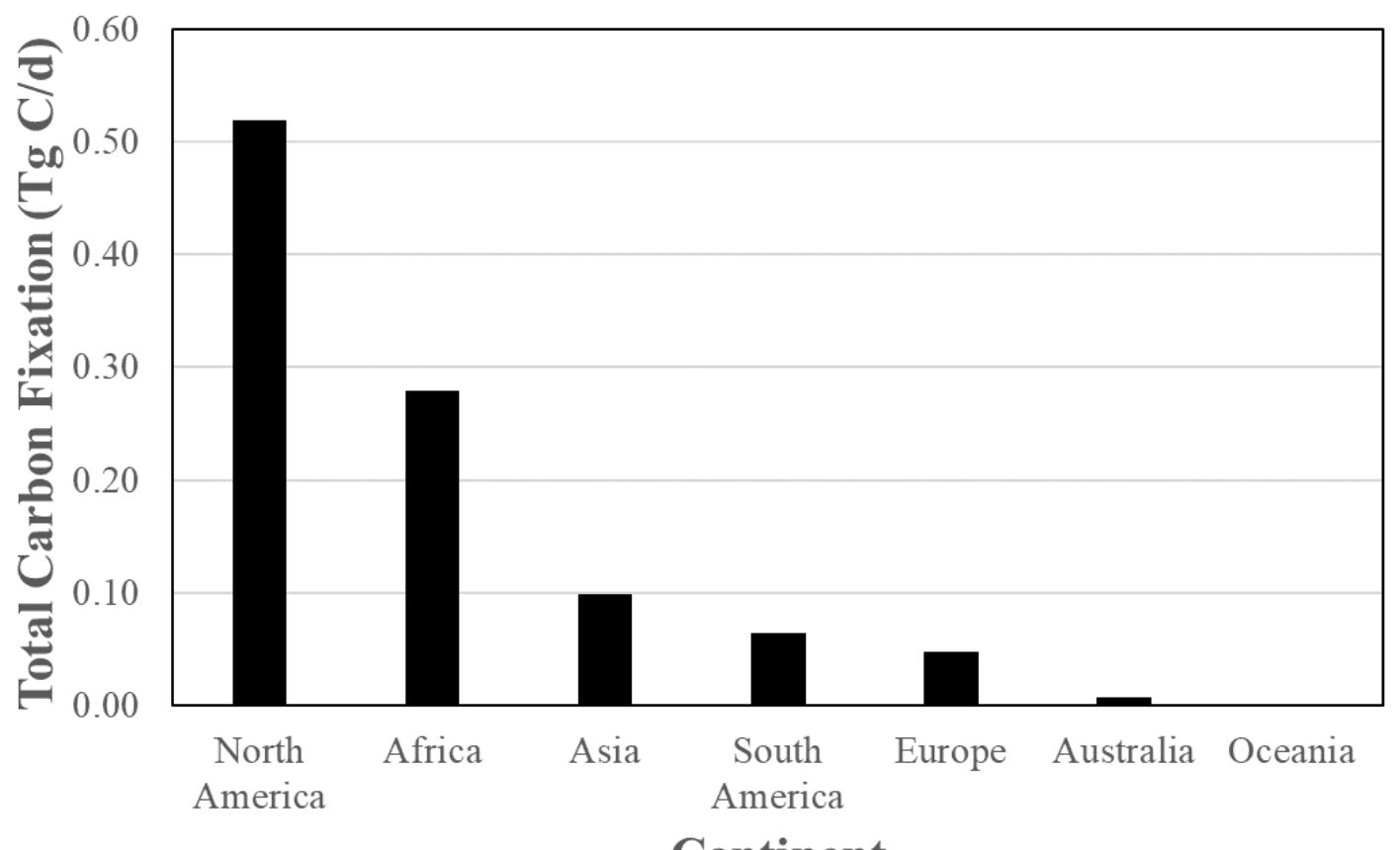

Continent

Figure 3.6. Total continental growing season carbon fixation $(\mathrm{Tg} \mathrm{C} / \mathrm{d})$. 
A generalized distribution of global freshwater carbon fixation by ecoregion is shown in Figure 3.7. Approximately $50 \%$ of the global carbon fixation is observed in nine ecoregions from North America and Africa (Laurentian Great Lakes, Lake Victoria Basin, English Winnipeg Lakes, Eastern Hudson, Bay Ungava, Southern Hudson Bay, Lake Chad, Western Hudson Bay, Lake Tanganyika, Upper Parana) while more than $25 \%$ ( $27 \%)$ of the global carbon fixation were from the Laurentian Great Lakes (14.5\%) and Lake Victoria Basin $(\sim 13 \%)$ alone. Unsurprisingly, very low carbon fixation is observed in the desert regions in Africa and North America. With the exception of the ecoregion areas encapsulating Lake Baikal and Tibetan Plateau, Asia experiences generally low total carbon fixation. Europe also has generally low total carbon fixation with the exception of Scandinavia.

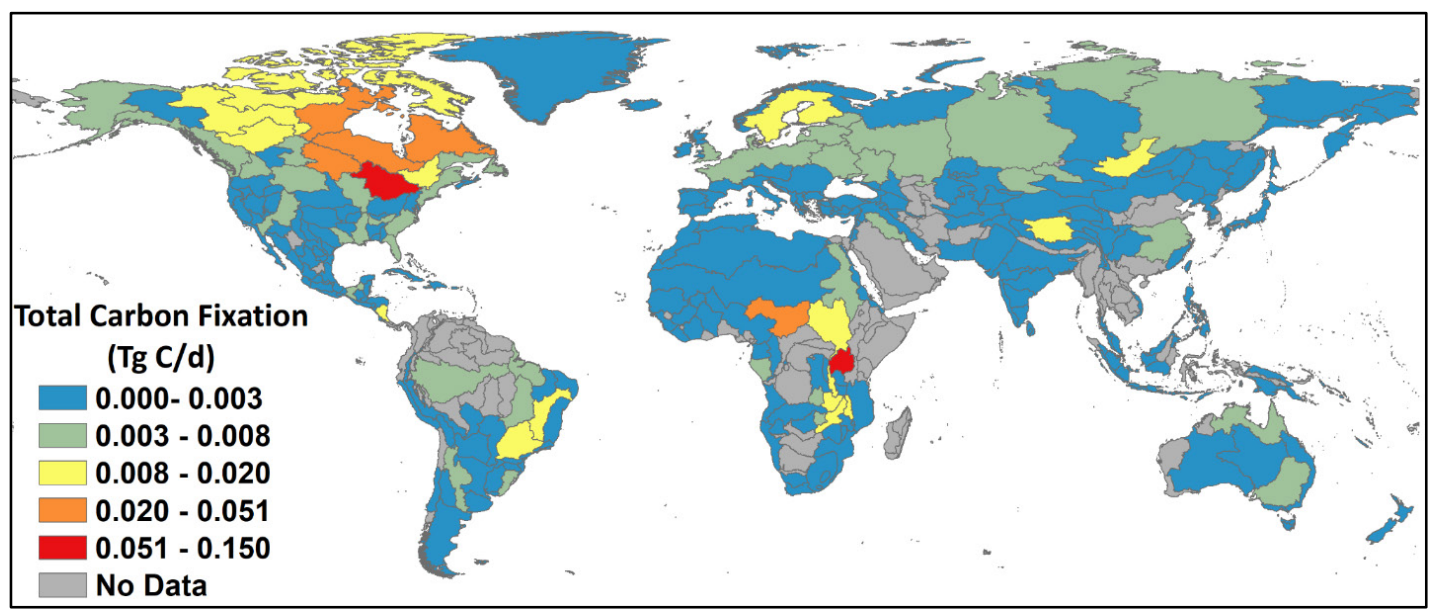

Figure 3.7. Total carbon fixation $(\mathrm{Tg} \mathrm{C} / \mathrm{d})$ from freshwater lakes aggregated by freshwater ecoregions of the world (Abell et al. 2008).

The relationship between total lake surface area and total carbon fixation (areal x lake surface area) was assessed by FEOW. A significant correlation between the $\log _{10}$ total surface area and $\log _{10}$ total carbon fixation was identified (Pearson's $r=0.37$, P-value $<0.005$ ). The relationship between total lake surface area and total fixation is shown in Figure 3.8. Points on the figure are colored by latitude regions in the northern and southern hemispheres $(0-30 \mathrm{~N}$ and $\mathrm{S}, 30-60 \mathrm{~N}$ and $\mathrm{S},>60 \mathrm{~N}$ degrees Note, no lakes in the southern hemisphere beyond 60 degrees $\mathrm{S}$ ). A general positive trend in increasing total fixation with 
total surface area is discernible but with high degree of variability. The high latitude lakes in the northern hemisphere are less productive, and appear to be more oligotrophic as they get larger. Ecoregions in the 0-30 latitude range in both northern and southern hemispheres and the 30-60 range in the southern follow a similar pattern, while ecoregions in the far northern latitudes deviate from the general trend (e.g. greater lake surface area with lower total carbon fixation in ecoregions $>60 \mathrm{~N}$ ). Significant differences between the mean total fixation ( $\log _{10}$ transformed) of each group were identified (ANOVA p-value $<0.005$ ). Ecoregions in the northern hemisphere $>60 \mathrm{~N}$ had significantly lower total fixation than all other latitude ranges. All other ecoregion groups were similar with the exception of the southern 0-30 and northern 30-60 which were different from each other. The results from these comparisons total freshwater surface area alone is unable to predict total freshwater carbon fixation particularly in high latitudes where areal carbon fixation rates are generally low.

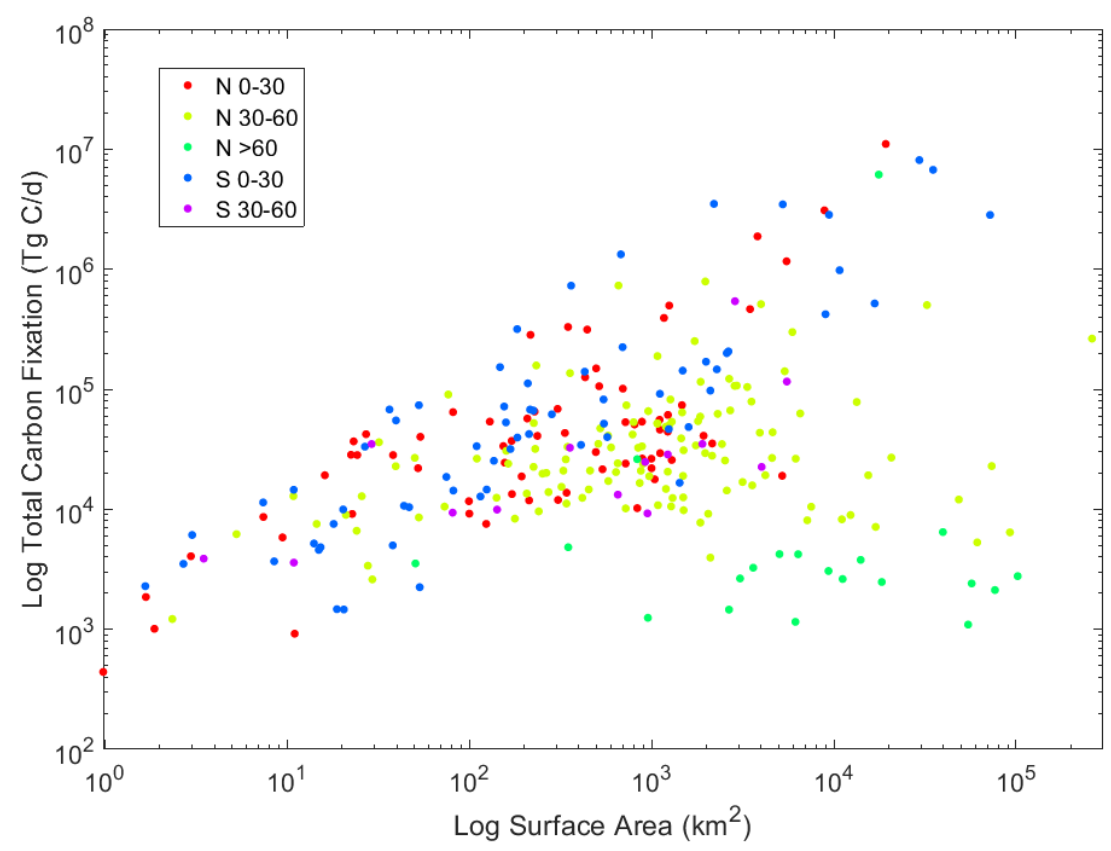

Figure 3.8. Log-log plots of total lake surface area and total carbon fixation for lakes aggregated by freshwater ecoregions of the world. Data points are colored by groups (Red - N 0-30 degrees, Yellow - N 30-60 degrees, Green $-\mathrm{N}>60$ degrees, Blue - S 0-30 degrees, Purple -S 30-60 degrees). 
When considering all $\sim 80,000$ lakes individually, significant log-log relationships were identified between lake total carbon fixation and lake size for both northern and southern hemispheres (northern hemisphere $y=1.1647 x+5.448$, $p$-value $<0.001, R^{2}=0.76$; southern hemisphere $\mathrm{y}=1.0658 \mathrm{x}+6.6793$, $\mathrm{p}$-value $<0.001, \mathrm{R}^{2}=0.90$ ). Figure 3.9 is a log-log plot depicting the relationship between lake area and total carbon fixation for the northern (top panel) and southern (bottom panel) hemispheres. From the figure it can be seen there is a higher degree of variability in total fixation for lakes of smaller size compared to the largest lakes in the northern hemisphere. For example, the coefficient of variation $(\mathrm{CoV})$ for lakes smaller than $1000 \mathrm{~km}^{2}$ is $\sim 2.5$-fold greater than that for lakes greater than $1000 \mathrm{~km}^{2}(\mathrm{CoV}$ for $>1000 \mathrm{~km}^{2}=2.1$; CoV for $<1000 \mathrm{~km}^{2}=5.4$ ). Conversely, for the southern hemisphere, similar $\mathrm{CoV}$ is observed between lakes greater and smaller than $1000 \mathrm{~km}^{2}$ (CoV for $>1000$ $\mathrm{km}^{2}=2.5$; $\mathrm{CoV}$ for $<1000 \mathrm{~km}^{2}=2.8$ ). Similar to the ecoregion comparison above, these results suggest that estimating global lake carbon fixation based on surface area alone can result in large errors particularly so for smaller lakes which are predominantly located in the far northern latitudes.
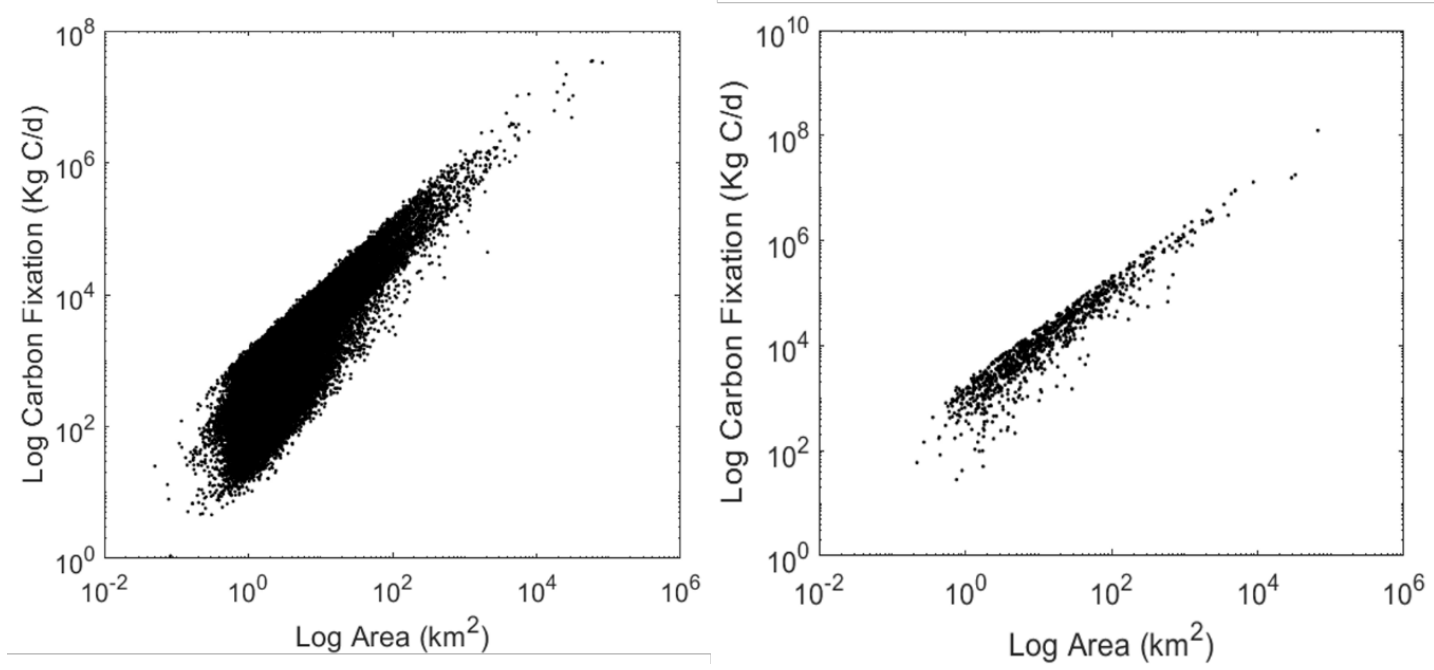

Figure 3.9. Log-log plots of individual lake area and total carbon fixation for all 80000 lakes grouped by hemisphere (northern - left panel, southern - right panel). 


\subsection{Discussion}

The use of remote sensing as a viable tool to estimate carbon fixation from freshwater lakes of the world using a straightforward DIM with an empirically estimated light utilization index was established in this study. This method requires very limited input parameters (i.e. chlorophyll and irradiance) that are readily available via remote sensing observations. More importantly the simple model we generated to estimate the light utilization index parameter from latitude allows for its broad application to study freshwater production anywhere in the world. Prior to this study, the use of remote sensing to estimate carbon fixation was limited to application in the marine environment (Behrenfeld and Falkowski et al. 1997) and several large freshwater lakes (Lohrenz et al. 2004, Shuchman et al. 2013, Soomets et al. 2015, Warner and Lesht 2016, Fahnenstiel et al. 2016). The methods used in those previous works were more complex requiring more robust in situ data sets for model parametrization according to their region of interest thus limiting their application across broad spatial scales (i.e. global). For example, Behrenfeld and Falkowski (1997) developed $\mathrm{a} 7^{\text {th }}$ order polynomial relationship between water temperature and the maximum water column carbon fixation rate $\left(\mathrm{P}^{\mathrm{B}}{ }_{\text {opt }}\right)$ using data measured throughout the global ocean. Similarly, Fahnenstiel et al. (2016) developed a $4^{\text {th }}$ order polynomial relationship between lake water temperature and the maximum carbon fixation rate at light saturation $\left(\mathrm{P}_{\max }\right)$ for data measured in the Laurentian Great Lakes. The calibration of these more sophisticated model approaches required significant numbers of co-located temperature and photosynthetic rate observations over the dynamic range of conditions to be considered in their region of interest. And since these measurements are severely lacking for freshwater systems, particularly for small remote systems, the use of these existing models for global studies is limited.

A unique aspect of this study is the first documented characterization of the light utilization index parameter for a variety of freshwater systems throughout the world. The light utilization index has been relatively well characterized in the marine environment by a variety of investigators (review in Platt 1986) exhibiting an effective range of 0.31-0.66 g 
$\mathrm{C}(\mathrm{g} \mathrm{Chl})^{-1} \mathrm{~m}^{2}(\mathrm{E})^{-1}$. The freshwater LUI values reported in this study exhibit a much larger range with maximum values more than 1.7 times larger than the marine values, however the mean values are quite similar. It is expected that LUI values in marine and freshwater systems would be similar as phytoplankton in both environments have similar photosynthetic efficiencies. It is likely the difference in the maximum LUI for marine and freshwater environments is due to the lack of marine observations from the low latitude ranges (e.g. tropics). Additionally, the LUI values reported by Platt (1986) were synthesized from individual study mean values, thus likely reducing the true range of LUI values encountered in the marine environment.

While this new method for estimating phytoplankton carbon fixation is straightforward to implement and very useful to global studies it is not without limitations. Because the carbon fixation rate is parametrized by latitude spanning the global range (0-90 degrees) with significant natural variability, it is likely large errors in production could be encountered for any individual lake. In light of these potential errors, this new approach should be implemented when little to know a priori information exists for any given lake system. This limitation, though, does not preclude one from developing their own parametrization of the LUI based on any number of predictive variables for their particular system of interest. However, if more complete bio-physical data exists for calibration (e.g. chlorophyll concentration, extinction coefficient, temperature, etc...), it is expected more sophisticated approaches such as the Great Lakes Production Model (GLPM) (Fee 1973, Fahnenstiel et al. 2016) or the Vertically Generalized Production Model (VGPM) (Behrenfeld and Falkowski 1997) are likely to result in more accurate production estimates.

A surprising observation from this study was that areal carbon fixation rates were not strongly related to lake area. Sayers et al. (2015), using limited comparisons $(\mathrm{n}=185)$ of remote sensing products, showed large lakes exhibited lower chlorophyll-a concentrations than medium and small lakes and since primary production is strongly dependent on chlorophyll concentrations we it would be reasonable to have expected large lakes to exhibit the lowest carbon fixation rate values. However, an extreme number of the smallest 
lakes are located in far northern latitudes where the light utilization index parameter is lowest resulting in lower carbon fixation rates than previously expected. While our observations differed from the limited comparison in Sayers et al. (2015), the results generally agree with those observed by Fee et al. (1992) for a set of variable sized Canadian shield lakes. The authors noted that areal production for smaller $(<1000 \mathrm{~km} 2)$ and larger lakes (>10000 km2) was generally lower than for medium sized lakes (1000-10000 km2) due to in part to increased chlorophyll biomass and not differing photosynthetic parameters. It is hypothesized that medium sized lakes generally exhibit higher areal production due to the combined offset of two parameters (temperature and turbulence) supporting increased phytoplankton growth. Our results do indicate that the smallest lakes have the lowest production rates compared to larger rates, However, we also observe very high rates in smaller lakes in both hemispheres. It is difficult to compare production rates by lake size over the full latitude range as the light utilization parameter also strongly influences production rates. The combined effects of variable chlorophyll concentration, light utilization rate, and irradiance across continents and hemispheres underscores the complexity in estimating lake production over large scales. Future work is needed to better understand the lake-size effect on carbon fixation rates for regions of similar photosynthetic efficiencies (i.e. similar latitude in our model).

The new remote sensing method reported here offers new insights into regional and local carbon fixation patterns that cannot be resolved with model based approaches. Historically, carbon fixation from global freshwater lakes has been estimated using model based approaches that assume the global total number of lakes and their size distribution. For example, Smith (2011) utilized a Monte Carlo simulation based approach to estimate global freshwater lake production accounting for deterministic and stochastic controlling factors and latitudinal models of lake size and abundance distributions. And while this approach is very useful for generating a total global freshwater production estimate for comparison with terrestrial and marine production, it lacks the ability to provide spatial information at regional and local scales throughout the world. Our new remote sensing based approach provides this granularity based on actual observations of individual lakes thus providing 
estimates that are more robust for any particular region in the world. This is a critical capability that is needed to understand how local factors control rates of production in lakes.

Application of the new DIM approach resulted in an improved understanding of global spatial variation of areal and total carbon fixation rates. Prior to this work, spatial comparisons of freshwater fixation rates have been made using spatially limited in situ measurements, often utilizing different techniques, making it difficult to draw quantitative conclusions (Fahnenstiel et al. 2016). Results from this study indicated that southern hemisphere lakes, particularly those in Africa, are generally more productive than lakes in the northern hemisphere for similar latitude ranges. We hypothesize the elevated production in southern hemisphere lakes is attributable to the increased trophic levels likely due to of human activity. This is supported for tropical latitude ranges (0-30 degrees) where the southern hemisphere mean chlorophyll concentration was almost 50\% larger than that of the northern hemisphere. Lakes in the southern hemisphere are generally more accessible than lakes in the northern hemisphere naturally inviting more human interaction thus generally increasing the availability of nutrients stimulating phytoplankton growth. Conversely, lakes in the northern hemisphere are highly concentrated in extreme northern latitudes that were previously glaciated (Raymond et al. 2013). The sheer remoteness of these lakes limits the impact from humans resulting in limited nutrient availability primarily controlled by the surrounding natural landscape (i.e. rock) Even northern hemisphere lakes at more moderate latitudes ( $<45$ degrees $N)$ are less inviting to human activity as they are generally located in more mountainous areas particularly in southern Asia and southern North America.

Knowing how much and where freshwater carbon fixation occurs is critical for improved understanding of Earth's carbon budget. Even though freshwater lakes account for only $4 \%$ of the surface of the Earth (Verpoorter et al. 2014) they are a significant contributor to the overall carbon cycle (Tranvik et al. 2009) thus underlying the importance of their accurate characterization. Freshwater carbon cycling models rely on parameterizations of 
the different modes $\mathrm{CO}_{2}$ is transported through a lake system. These parameterizations are critical for producing accurate estimates of carbon flux and ultimately determine whether a given lake is a carbon sink or a carbon source (Engel et al. 2018). The new carbon fixation data set produced in this study can fill a gap in these parameterizations that have previously assumed "all lakes on Earth function similarly" (Engel et al. 2018). With improved estimates of photosynthetic rates for many lakes in the world, modelers can begin to realize the magnitude of the effect lake composition has on flux calculations. Moreover, the spatial context of the new remote sensing freshwater products provides the opportunity to study regional carbon cycling dynamics that were previously lacking a key component (i.e. lake carbon fixation). Assessing these effects over time may also shed light into what the future may in the face of climate change and anthropogenic forcing (Engel et al. 2018).

\subsection{Concluding Remarks}

This work resulted in the first remote sensing derived estimates of global freshwater lake phytoplankton carbon fixation which represents a crucial first step toward fully understanding global lake carbon budgets. The straightforward DIM presented here is valuable tool to estimate freshwater phytoplankton production anywhere in the world with only minimal remote sensing or in situ data. This flexibility allows for a truly global analysis of carbon fixation rates which can be assessed relative to regional and local scale anthropogenic forcing which is not possible with current model based approaches. Additionally, future implementation of the DIM approach to freshwater remote sensing time-series observations will likely provide valuable and descriptive insights into the role climate change has on regional and global carbon cycle dynamics. 


\section{Cyanobacteria blooms in three eutrophic basins of the Great Lakes: A comparative analysis using satellite remote sensing}

Blooms of harmful cyanobacteria (cyanoHABs) were mapped for three eutrophic basins (western basin of Lake Erie-WBLE, Green Bay, Lake Michigan-GB, and Saginaw Bay, Lake Huron-SB) in the Great Lakes from 2002-2013 using MODIS ocean colour imagery. These blooms were examined in relationship to basic meteorological and environmental parameters. Annual cyanoHAB extent trends were generated using two modified remote sensing approaches. The first approach was a modified bio-optical chlorophyll retrieval algorithm enhanced with empirical relationships to estimate water column cyanoHABs $(\mathrm{MCH})$ whereas the second approach uses near-infrared reflectance to quantify surface scums of cyanoHABs (SSI). The development and application of the SSI is a unique product in the Great Lakes and may have generic application to ecological and public health issues. Satellite derived cyanoHAB estimates agreed well with in situ observations (89\% accuracy). The annual cyanoHAB trends (MCH and SSI) for WBLE, SB, and GB were not similar for the 2002-2013 analysis period. A recent trend of increasing cyanoHABs was noted in WBLE but not in GB or SB. Moreover, extensive and persistent surface scums were observed in WBLE but not in GB or SB. Meteorological parameters were similar among the basins, however significant differences in spring discharge of the dominant river were observed among basins. Spring discharge was a significant predictor of cyanoHAB occurrence in WBLE but not in GB and SB. Wind induced sediment re-suspension events were common during the bloom period in WBLE but not GB or SB and these events were highly correlated with cyanoHAB occurrence. The differences among basins in the role of riverine discharge and resuspension suggests local factors are more important than regional factors in controlling cyanoHAB dynamics within these three basins in the Great Lakes.

Keywords: MODIS; water quality; Cyanobacteria, chlorophyll a, Great Lakes 


\subsection{Introduction}

Colonial cyanobacteria (blue-green algae) commonly occur in many eutrophic regions throughout the world (Bianchi et al. 2000; Vahtera et al. 2007; Joehnk et al. 2008; Qin et al. 2010; Schindler et al. 2012). When conditions are suitable large blooms of cyanobacteria may be common in these waters. Excessive loading of phosphorus and other nutrients from rivers and streams play an important role in bloom occurrence as well as prolonged durations of sunlight and warm temperatures (Robarts and Zohary 1987; Rapala et al. 1997; Ibelings et al. 2003; Kanoshina et al. 2003; Paerl et al. 2011). Some cyanobacteria produce toxins (Sivonen 1996; Codd et al. 2005) and toxin concentrations in cyanobacteria blooms (cyanoHAB) may have serious potential negative impacts on aquatic resources, public health, and community economics (Rinta-Kanto et al. 2005; Ouellette et al. 2006). Evidence suggests cyanoHAB bloom occurrences are increasing worldwide in both freshwater lakes and inland seas (Otten and Paerl 2011). One unique trait of cyanoHABs is their ability to form surface scums due to vacuolation (van Rijn and Shilo 1985; Klemer et al. 1996). Scums have been linked to very high concentrations of toxins and are easy to come into contact with during recreational activities (i.e. boating, fishing, etc.), thus making their detection a potentially important aspect of any cyanobacterial monitoring program (Bartram and Rees 1999).

One species of cyanobacteria that is capable of producing large toxic blooms is Microcystis aeruginosa. Blooms of this cyanobacterium are re-occurring annual events within eutrophic waters of the Great Lakes most notably in the western basin of Lake Erie (WBLE) (Vanderploeg et al. 2001; Bridgeman et al. 2013), but also in Saginaw Bay (SB) in Lake Huron (Fahnenstiel et al. 2008), and Green Bay (GB) in Lake Michigan (DeStasio et al. 2008) (Figure 4.1). These basins are similar in that each has a dominant river in which a large percentage of the total basin-wide nutrient load is delivered (Maumee River-WBLE, Fox River-GB, and Saginaw River-SB). While all three of these basins are shallow, eutrophic bodies of water within a larger freshwater system, the historical patterns of cyanoHAB blooms across these three regions have not been examined. Lake Erie has 
exhibited an increasing trend in $\mathrm{HAB}$ occurrences starting in the 2000s culminating in the largest bloom ever recorded in 2011 (Michalak et al. 2013; Bridgeman et al. 2013). The NOAA experimental HAB bulletin has indicated an even larger bloom may have taken place in 2015 (http://www.glerl.noaa.gov/res/HABs_and_Hypoxia/lakeErieHABArchive/). The mid1990s saw the reoccurrence of large blooms of cyanobacteria in Saginaw Bay concurrent with the wide-scale establishment of invasive zebra mussels, and continue to be observed in the late summer period (Bierman et al. 2005; Millie et al. 2009;). Green Bay has seen a recent change in the phytoplankton community toward a more frequent dominance of cyanobacteria following the invasion of zebra mussels in 1992 (De Stasio et al. 2008). While cyanoHABs have been documented in all three basins in the Great Lakes, more than a decade of extensive in situ and satellite based monitoring in WBLE has led to a functional understanding of annual cyanoHAB dynamics within this basin (Bridgeman et al. 2013; Michalak et al. 2013; Obenour et al. 2015,). Much less is known about annual cyanoHAB occurrence trends and controlling factors in GB and SB. 


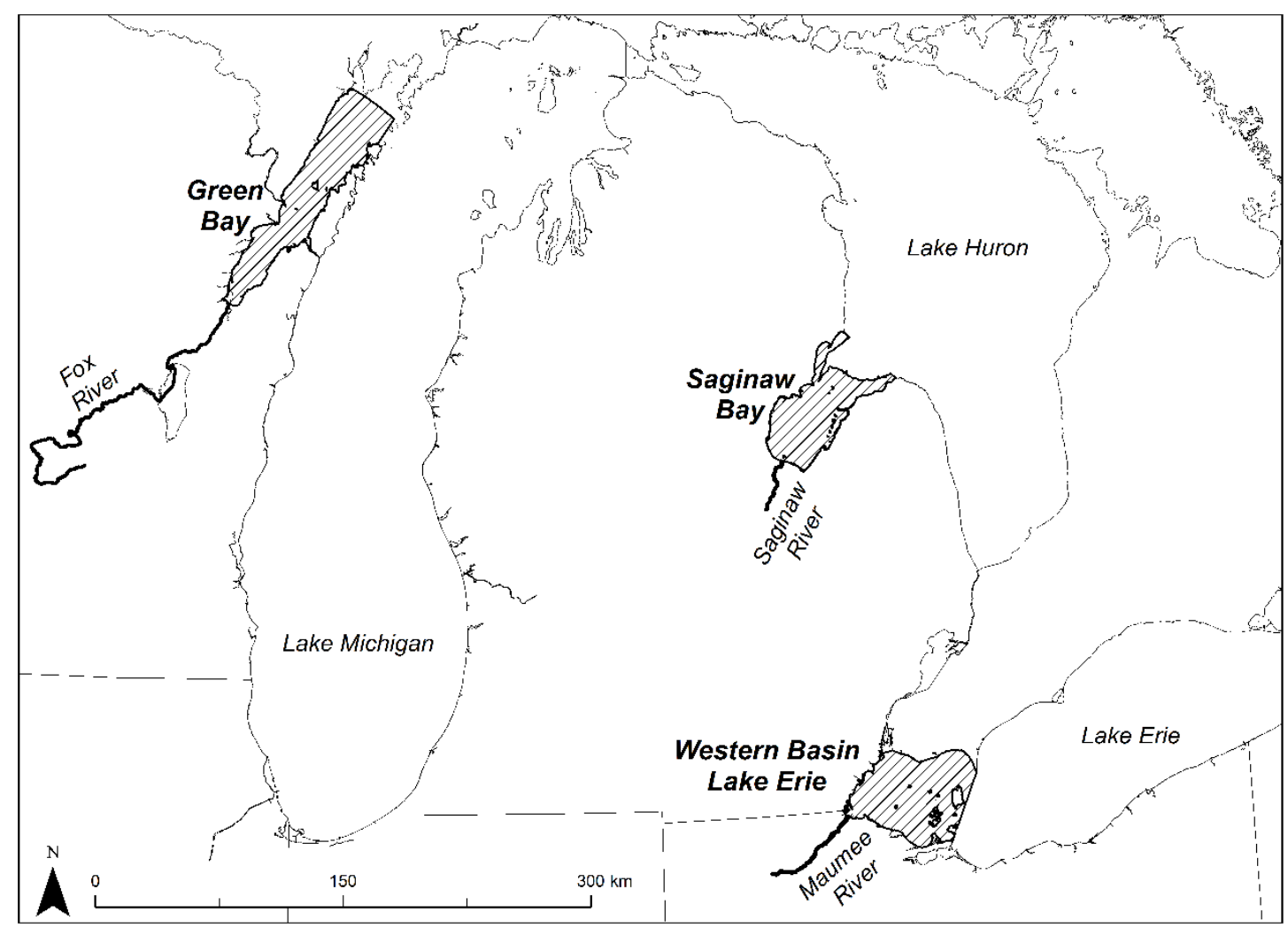

Figure 4.1. Map of areas analyzed for cyanoHAB occurrences in the Great Lakes. Hatched areas indicate the basins included in analysis. The dominant river (nutrient source) for each basin is also labeled on the map.

Satellite remote sensing provides the potential for long- term spatial and temporal synoptic monitoring of cyanoHAB events in the Great Lakes, and thus can help quantify the trends in cyanoHABs. This basin-scale mapping potential has resulted in the development of cyanobacteria satellite mapping algorithms that are sensitive to the abundance of cyanobacteria and pigments other than chlorophyll and the unique near surface abundance associated with cyanobacteria blooms. One such pigment, phycocyanin, is found in cyanobacteria and has been used to document the presence of cyanobacteria from remote sensing (Gons et al. 1991; Dekker et al. 1993; Simis et al. 2005; Kutser et al. 2004, 2009). The ability to discriminate blue-green algae from other bloom forming algae is critical to 
distinguishing cyanobacteria blooms. Wynne et al. (2008) developed an approach based on the Fluorescence Line Height (FLH) algorithm (Abbott and Letelier 1999) that exploits the spectral shape of cyanobacteria absorption features in the red and "red edge" portions of the electromagnetic spectrum using MERIS satellite imagery. Stumpf et al. (2012) used this approach to further quantify cyanobacteria blooms in the WBLE from 2002-2011 and relate annual bloom extent to river discharge. With the failure of MERIS in April 2012 new approaches using operational satellite systems are required to continue important cyanoHAB monitoring activities throughout the Great Lakes and other regions.

The overall goal of this study is to characterize annual historical trends of cyanoHABs in the three basins (WBLE, SB, and GB) in the Great Lakes from 2002-2013. These trends will be used to determine if they are similar or dissimilar among basins as well as explore basic relationships with a number of possible controlling factors including temperature, precipitation, river discharge, resuspension, and watershed land cover. These comparisons might provide useful information on the relative roles of regional (climate) or local (nutrient) control of cyanoHABs in the Great Lakes. In WBLE, the recent increase in cyanoHABs has been linked to nutrient loading and climate change (Stumpf et al. 2011; Michalak et al. 2013). A comparison of cyanoHABs in areas of the same geographic region (Great Lakes) may provide insights in the role of regional climate since the three study areas are in same region, and relatively similar climatic effects have been noted in this region (McCormick and Fahnenstiel 1999). Thus, if regional climate change effects are driving recent trends in cyanoHABs in WBLE we would expect to see relatively similar trends in GB and SB. On the other hand, if local factors are controlling cyanoHABs in areas of the Great Lakes we might expect recent trends in cyanoHABs in WBLE, GB, and $\mathrm{SB}$ to be more dissimilar. While regional climate factors may contribute to cyanoHABs in some areas of the Great Lakes, we believe that local factors are more important in controlling cyanoHABs in the Great Lakes. We hypothesize that recent trends in cyanoHABs will not be similar among areas of the Great Lakes, and that local factors will explain a significant amount of annual variability within any basin. A better understanding of the controlling factors that drive cyanoHAB occurrences can lead to better adaptive 
management remediation efforts. To evaluate these trends and relationships with key variables we used two satellite based approaches. The first was a modified chlorophyll algorithm supplemented with empirical relationships to detect cyanoHAB blooms in the Great Lakes. The second approach used a near-infrared surface reflectance method to determine the extent of cyanoHAB surface scums. This second approach is novel in the Great Lakes and has the potential for wide spread generic use, across sensor platforms, which may include utility in early public health warning systems.

\subsection{Methods}

\subsubsection{Field and environmental data}

Discrete samples from the near surface region $(0-1 \mathrm{~m})$ were taken with a modified clean Niskin bottle (Fitzwater et al. 1982; Fahnenstiel et al. 2002). Surface scums were sampled with $500 \mathrm{ml}$ Nalgene bottles used horizontally to capture the surface scum. Once back in the lab, the contents of the Nalgene bottle were placed in high light incubator and scums were allowed to reform at the top of the bottle at which time they were carefully sampled with a syringe. Samples for pigment analyses were filtered onto Whatman GF/F filters, extracted with appropriate solvent, and analyzed fluorometrically. For chlorophyll $a$ samples filters were extracted with N, N-dimethylformamide (Speziale et al. 1984) and analyzed on a Turner Designs fluorometer calibrated with chlorophyll- $a$ standards. Filters for phycocyanin determination were extracted in phosphate buffer (Ricca Chemical, $\mathrm{pH}$ 6.8) using two freeze-thaw cycles followed by sonication (Horváth et al. 2013). Relative fluorescence was measured on a Turner Aquafluor fluorometer and converted to phycocyanin concentration using a series of dilutions of a commercial standard (SigmaAlderich).

Air temperature and precipitation data was obtained from airport records near western basin Lake Erie (KTOL), Green Bay (KGRB), and Saginaw Bay (KMBS). Monthly and annual mean values were created from daily measurements. Mean river discharge values 
for the dominant rivers in proximity of the cyanoHABs (Maumee River-WBLE, Fox RiverGB, and Saginaw River-SB; see Figure 4.1) were generated from daily observations provided by the USGS (http://waterdata.usgs.gov/nwis/rt).

Wind induced sediment resuspension events were calculated using the USACE Wave Information Studies (WIS) hindcast wave climatology data. The WIS wave data includes significant wave height, period, and direction for a densely spaced series of "virtual wave gauges" at various depth ranges throughout the Great Lakes. For this analysis, wave data stations in WBLE, GB, and SB were selected that correspond to areas where cyanoHAB occurrences were most frequent (i.e., Maumee Bay, southeast part SB, southwest part GB). The stations used in this analysis were 92112 in WBLE, 94163 in GB, and 93050 in SB. Daily mean WIS wave parameters from 2002-2013 were derived from hourly observations, and used to calculate bottom shear stresses following the model from Schwab et al. (2006). A sediment resuspension event was defined as when the horizontal stress at the bottom of the water column exceeded 0.1 Pa (Fukuda and Lick 1980). The number of days where bottom stress exceeded 0.1 Pa were recorded for the July-September period for each of the three basins.

\subsubsection{CyanoHAB mapping and detection}

Two approaches were used to map cyanoHABs in the Great Lakes using MODIS ocean colour satellite data. The first approach uses a modification of the CPA-A algorithm (Shuchman et al. 2006, 2013a) with two empirical relationships to link specific high chlorophyll- $a$ concentrations to likely cyanoHABs (modified CPA/HAB approach). The second approach involves the application of a cyanoHABs scum index that detects the presence of cyanobacteria surface scums (surface scum index).

The semi-analytical Color Producing Agent (CPA) algorithm (Shuchman et al. 2013) that accurately derives accurate chlorophyll- $a$ concentrations in the Great Lakes was modified and implemented to map HAB events from 2002-2013. The CPA Algorithm (CPA-A) simultaneously retrieves concentrations of chlorophyll $a$, suspended minerals (SM), and 
coloured dissolved organic matter (CDOM) from satellite reflectance imagery through the use of lake specific hydro-optical models. The hydro-optical models are the average optical cross sections of chlorophyll, SM, and CDOM absorption as well as chlorophyll and SM backscatter across the visible spectrum. The hydro-optical model values used in this study were derived from extensive inherent optical property and in situ water chemistry measurements made in Lake Erie during the historical bloom season and are reported in Shuchman et al. (2013). The CPA-A was successfully validated in the Great Lakes (Shuchman et al. 2013a), including phytoplankton rich Lake Erie, thus verifying the usefulness of this approach in accurately retrieving chlorophyll $a$ concentration from MODIS.

Because the CPA-A does not directly detect blue-green algae abundance, a modified approach is needed to determine the presence of cyanobacteria blooms. We chose an approach that utilized the CPA-A, but was supplemented with empirical relationships between other environmental variables and chlorophyll $a$ to predict cyanobacteria abundance. It must be pointed out that the empirical relationships developed in this study are specific to the Great Lakes, and may not have broad application in other regions. Cyanobacteria photopigment, phycocyanin, is related to chlorophyll $a$ concentration in WBLE. Measurements of surface chlorophyll- $a$ and phycocyanin concentrations from WBLE in the 2011 bloom period (July-October) were compared to identify a quantitative relationship between the two pigments (NOAA/GLERL data). Figure 4.2 is a scatterplot of chlorophyll $a$ (x-axis) versus phycocyanin (y-axis). Clearly observable from the plot, there are very small quantities $\left(<5 \mathrm{mg} \mathrm{m}^{-3}\right)$ of phycocyanin detected at chlorophyll- $a$ concentrations less than approximately $18 \mathrm{mg} \mathrm{m}^{-3}$, while large quantities of phycocyanin (5-300 $\mathrm{mg} \mathrm{m}^{-3}$ ) were observed when chlorophyll $a$ concentrations were $>18 \mathrm{mg} \mathrm{m}^{-3}$. These relationships suggest high chlorophyll concentrations are generally associated with cyanobacteria blooms. A segmented regression analysis (SegReg http://www.waterlog.info/segreg.htm) was performed to determine the appropriateness of a chlorophyll $a$ threshold value of $18 \mathrm{mg} \mathrm{m}^{-3}$ for cyanoHAB classification. The segmented regression analysis results indicated a statistically significant breakpoint threshold between 
12 and $20 \mathrm{mg} \mathrm{m}^{-3}$ of chlorophyll $a$ (segmented model coefficient of explanation (0.78) greater than non-segmented model coefficient of determination, $R^{2}(0.77)$ ). Thus, a threshold of $18 \mathrm{mg} \mathrm{m}^{-3}$ chlorophyll $a$ was used to determine cyanoHAB blooms. A second empirical relationship was used to further limit the probability of any false positive cyanobacteria identification. While high chlorophyll- $a$ concentrations in Lake Erie are often associated with cyanobacteria, diatoms and large green algae can also exhibit large chlorophyll concentrations (Munawar and Munawar 1976; Millie et al. 2009). These other algae often occur early or late in the vegetative growing season when water temperatures are cooler than $20^{\circ} \mathrm{C}$, whereas cyanobacteria prefer warmer temperatures (Millie et al. 2009). Data collected from NOAA/GLERL in 2005-2012 showed no significant cyanobacteria blooms in WBLE, GB or SB when water temperatures were $<20^{\circ} \mathrm{C}$. This relationship is supported by the work of Stumpf et al. (2012) where no significant cyanobacterial blooms in Lake Erie prior to June were noted when mean water temperatures were $<20^{\circ} \mathrm{C}$. Thus, using these two relationships between cyanobacteria and environmental variables, our modified CPA-A HABs $(\mathrm{MCH})$ approach was used to detect cyanobacteria blooms in the Great Lakes. 


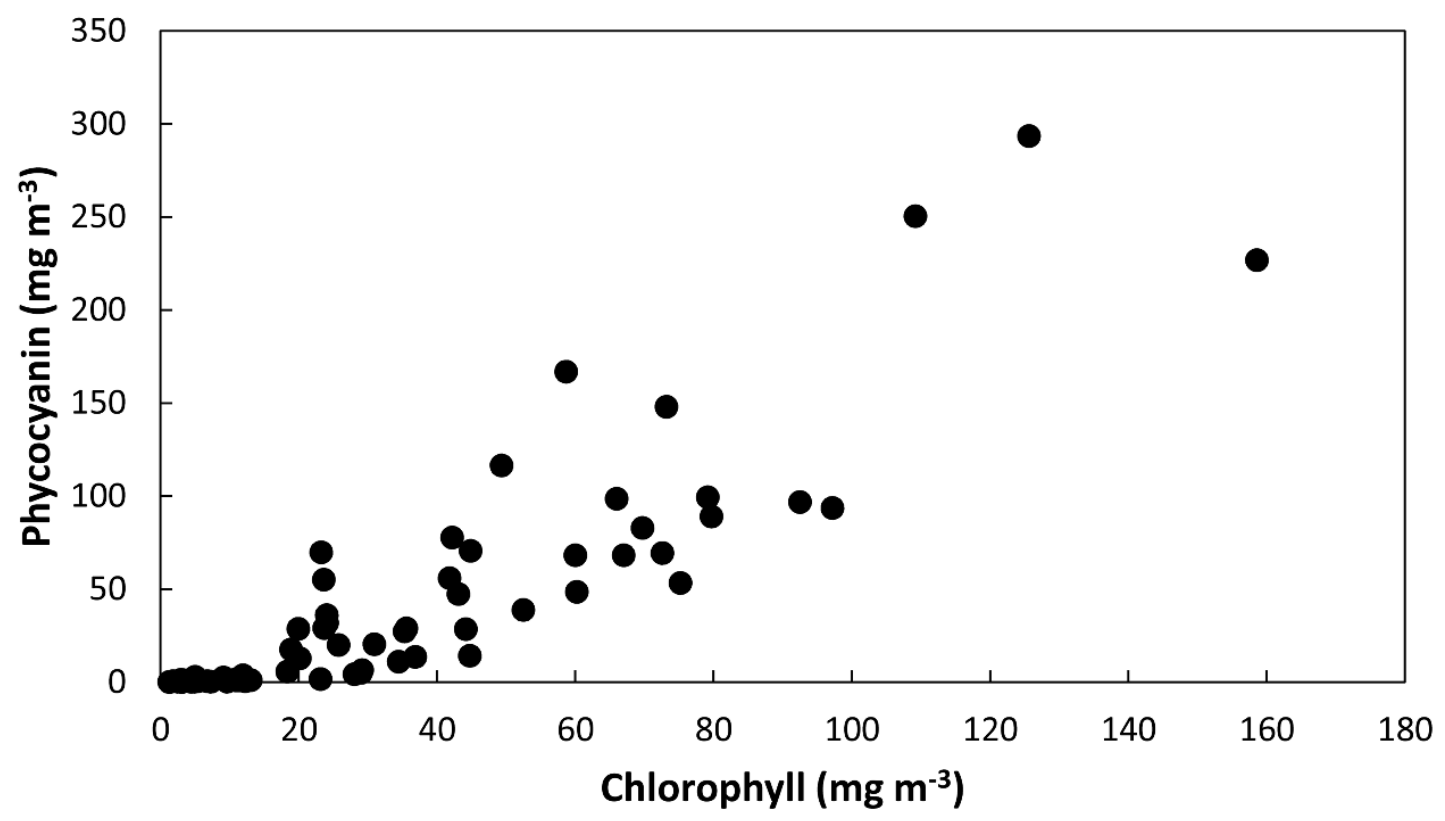

Figure 4.2. Surface chlorophyll $a$ concentration vs. phycocyanin concentration measured in WBLE during the 2011 July-October bloom period.

The second approach used to map cyanoHABs in the Great Lakes was a surface scum index (SSI). A surface scum measure is important as extensive sampling in Lake Erie and elsewhere has indicated that extremely large concentrations of Microcystis (i.e. those effecting public health) are often found in floating algae mats or surface scums (Zohary and Robarts 1990) which are associated with high toxin concentrations (Chorus and Bartram 1999, Carmichael 2001, NOAA/GLERL data). Historically, traditional chlorophyll mapping algorithms have not been able to simultaneously retrieve both surface algal scum and water column phytoplankton due to optical water complexity and imperfect atmospheric correction routines (Kutser 2009). To address this important public health issue, a methodology has been developed that can detect surface scum occurrences independent of chlorophyll concentration and may be applied to a variety of sensing platforms, including unmanned aerial vehicles, with limited sets of available wavelengths. This approach uses the near infrared portion of the electro-magnetic spectrum to determine if scums are located at the water surface. Similar methods have been implemented to 
observe surface blooms in other freshwater lakes (Mathews et al. 2012; Hu et al. 2009, 2010; Peng et al. 2008), most notably Lake Taihu in China; however, several of these approaches rely on specific sensor bands (i.e. MERIS 709 and $754 \mathrm{~nm}$ bands, Mathews et al. 2012) not available to MODIS or require more rigorous scattering corrections (Hu et al. 2009) that are not as well understood in the Great Lakes region as on the open ocean. For the simple classification of surface scum presence, we elected to use the simple SSI approach as no tuning is required to produce time-series estimates for the three basins of interest and it has more generic application.

The SSI is an implementation of the Normalized Difference Vegetation Index which is a commonly used method in terrestrial vegetation biomass estimation. Solar radiation in the visible spectral region $(400-700 \mathrm{~nm})$ is absorbed by green vegetation as a source of energy in the process of photosynthesis. Conversely, NIR (wavelength $>700 \mathrm{~nm}$ ) radiation is predominantly scattered by green vegetation as the energy level per photon in this spectral region is insufficient for photosynthesis (Tenhunen et al. 1980). These fundamental properties are exploited in Equation (1):

$$
\mathrm{SSI}=\left(\frac{(\mathrm{NIR})-(\mathrm{VIS})}{(\mathrm{NIR})+(\mathrm{VIS})}\right)>0
$$

Where NIR is the spectral reflectance in a NIR wavelength and VIS is the reflectance in a visible wavelength, usually red. Note only positive values of the ratio are classified as a surface scum, thus the SSI values range from 0 to 1 . Moreover, near infrared radiation is also highly absorbent in water making the distinction between surface algae scum (filaments above or in close proximity to the air water interface) and all other water constituents apparent in SSI values. The new SSI maps positive values as surface scum by exploiting the observed high reflectance in the NIR relative to the red reflectance.

These two HAB mapping approaches (MCH and SSI) use MODIS satellite imagery provided freely from the NASA Ocean Bio-geochemical Processing Group (http://oceancolor.gsfc.nasa.gov/). MODIS bands 9-13 (band centers: 443, 488, 531, 547, 
$667 \mathrm{~nm})$ were used in the MCH and MODIS bands $1(645 \mathrm{~nm})$ and $2(858 \mathrm{~nm})$ were used in the SSI. All cloud free (>75\%) images were acquired for the three basins for the 20022013 period. MODIS Level1A data was acquired and processed to Level2 using SeaDAS (V6.4). A fixed model pair aerosol correction approach was applied to the images to ensure high NIR reflectance due to scum was not interpreted as atmospheric aerosols. Resulting atmospherically corrected reflectance data was compared to several in situ reflectance measurements (Shuchman et al. 2013a) with good agreement indicating this method produces acceptable reflectance data for cyanoHAB mapping in Great Lakes waters.

The $\mathrm{MCH}$ and SSI were applied to all images. The resulting classified images were summarized to produce annual extent statistics. Note for the analysis presented in this manuscript, the $\mathrm{MCH}$ and SSI provide the presence and absence of cyanoHABs and are not quantitative estimates of bloom abundance. The classified images were also summed each year to produce cyanoHAB heat maps which delineate the number of days cyanoHABs occurred at any given location in each of the three basins.

\subsubsection{Validation of cyanoHAB mapping method}

The two cyanoHABs mapping approaches (MCH and SSI) were evaluated by comparing field observations of cyanoHAB events in WBLE, GB, and SB. The accuracy of these approaches was evaluated on a pixel by pixel basis using comparisons between the two satellite HABs mapping approaches and in situ sampling. MODIS satellite images were identified and obtained within +/- 24 hours of a given field observation. This near coincident data set ensures a reasonable comparison can be made between in situ and satellite observations.

These selected images were processed with the $\mathrm{MCH}$ approach. The output maps were queried at each matching sampling station using a 3-by-3 pixel average surrounding the actual sampling point. The spatial averaging was applied to account for cyanoHAB movement between the field sampling time and image acquisition time. The retrieved satellite classification type (cyanoHAB or No cyanoHAB) was compared to the measured 
chlorophyll value and presence of cyanobacteria bloom at each corresponding site. If the satellite retrieval indicated cyanoHAB and the in situ data reported chlorophyll $a$ values > $18 \mathrm{mg} \mathrm{m}^{-3}$ with cyanobacteria bloom noted from field sampling, then the modified CPA algorithm was deemed successful. The algorithm was also considered successful if the satellite retrieval indicated no cyanoHAB presence, field chlorophyll value was $<18 \mathrm{mg}$ $\mathrm{m}^{-3}$ and no cyanobacteria bloom was noted in field samples. During the five sampling years $(2008,2009,2010,2011$, and 2013) there were 24 matches between sampling dates and clear satellite images in WBLE (11 days), GB (2 days), and SB (11 days). Sampling dates used in this comparison ranged from July-October. These matches produced 118 sampling station point comparisons to evaluate the modified $\mathrm{MCH}$ mapping approach in all three basins.

The new SSI was evaluated using data sets collected from WBLE in 2011 and 2013 and GB in 2012. There was no in situ scum data available for comparison in SB during the study period. There were a total of 5 satellite images coincident with in situ scum (radiometer indicated) observations producing 40 matchups. Sampling sites with positive SSI values and in situ scums were compared to the satellite-derived surface scum image using a 3-by-3 window.

Standard statistical analysis (Spearman Rank correlation and ANOVA with post-hoc analysis-TukeyKramer) was used for all comparisons (MATLAB). Statistical significance was set at alpha $=0.05$.

\subsection{Results}

In order to determine the applicability of the new approaches for mapping cyanoHAB extent in the Great Lakes, the output (MCH and SSI) was validated against in situ observations in WBLE, GB, and SB. Our new cyanoHABs mapping products agreed well with in situ cyanoHAB observations. The $\mathrm{MCH}$ approach performed well both when cyanoHABs were present (88\%) and not present (86\%), producing an overall mapping accuracy of $87 \%$ and Kappa of $83 \%$ with the majority (9) of misclassifications representing 
an over prediction of $\mathrm{HAB}$ presence by $\mathrm{MCH}$ (Table 4.1). There were only six occasions where the satellite $\mathrm{MCH}$ failed to correctly classify as a cyanoHAB with respect to the field data. The misclassifications were likely due to mixed pixels as the misclassified points were near the edge of the algal bloom. The validation misclassification due to mixed pixels was and issue in the period of early bloom initiation (June/early July) and much less so during period of bloom maximum extent (August- early October). The SSI approach also performed well when cyanoHAB scums were present $(85 \%)$ and not present $(96 \%)$ yielding an overall mapping accuracy of 93\% and Kappa of 74\% (Table 4.2). This successful validation demonstrates the applicability of our approaches to map cyanoHAB occurrences in the Great Lakes.

Table 4.1. Classification error matrix for $\mathrm{MCH}$ vs. in situ observations. $\mathrm{MCH}$ correctly classified 103 out of 118 comparisons for an overall accuracy of $87 \%$ and Kappa of 0.74 .

\begin{tabular}{|c|c|c|c|c|c|}
\hline \multirow{6}{*}{$\frac{T}{S}$} & \multicolumn{5}{|c|}{ In Situ } \\
\hline & & HAB & No HAB & Total & $\begin{array}{c}\text { Users } \\
\text { accuracy (\%) }\end{array}$ \\
\hline & HAB & 46 & 9 & 55 & 84 \\
\hline & No HAB & 6 & 57 & 63 & 90 \\
\hline & Total & 52 & 66 & 118 & \\
\hline & $\begin{array}{l}\text { Producers } \\
\text { accuracy (\%) }\end{array}$ & 88 & 86 & $\begin{array}{c}\text { Overall accuracy }(\%)=87 \\
\text { Kappa }=0.74\end{array}$ & \\
\hline
\end{tabular}

Table 4.2. Classification error matrix for SSI vs. in situ observations. SSI correctly classified 37 out of 40 comparisons for an overall accuracy of 93\% and Kappa of 0.83 .

\begin{tabular}{|c|c|c|c|c|c|}
\hline \multirow{6}{*}{ फे } & \multicolumn{5}{|c|}{ In Situ } \\
\hline & & Scum & No Scum & Total & $\begin{array}{c}\text { Users } \\
\text { accuracy (\%) }\end{array}$ \\
\hline & Scum & 11 & 1 & 12 & 92 \\
\hline & No Scum & 2 & 26 & 28 & 93 \\
\hline & Total & 13 & 27 & 40 & \\
\hline & $\begin{array}{c}\text { Producers } \\
\text { accuracy (\%) }\end{array}$ & 85 & 96 & $\begin{array}{c}\text { Overall accuracy }(\%)=93 \\
\text { Kappa }=0.83\end{array}$ & \\
\hline
\end{tabular}


The annual cyanoHAB extent trends (derived from MCH) for WBLE, SB, and GB were not similar for the 2002-2013 period (all $r<0.10$, all $p>0.05$ ). The recent increasing trend in cyanoHAB occurrence observed in WBLE was not seen in GB or SB (Figure 4.3). In WBLE the maximum extent of cyanoHABs occurred in 2011 and 2013 whereas in SB the maximum extent occurred in 2002 and 2006 and in GB the maximum extent occurred in 2002 and 2012 (Figure 4.3). CyanoHAB surface scums were more common in WBLE than in $\mathrm{SB}$ and GB where SSI values never exceeded $8 \mathrm{~km}^{2}$ (Figure 4.4). In Lake Erie the maximum SSI values occurred in the same years as the MCH maximum, 2011 and 2013. 

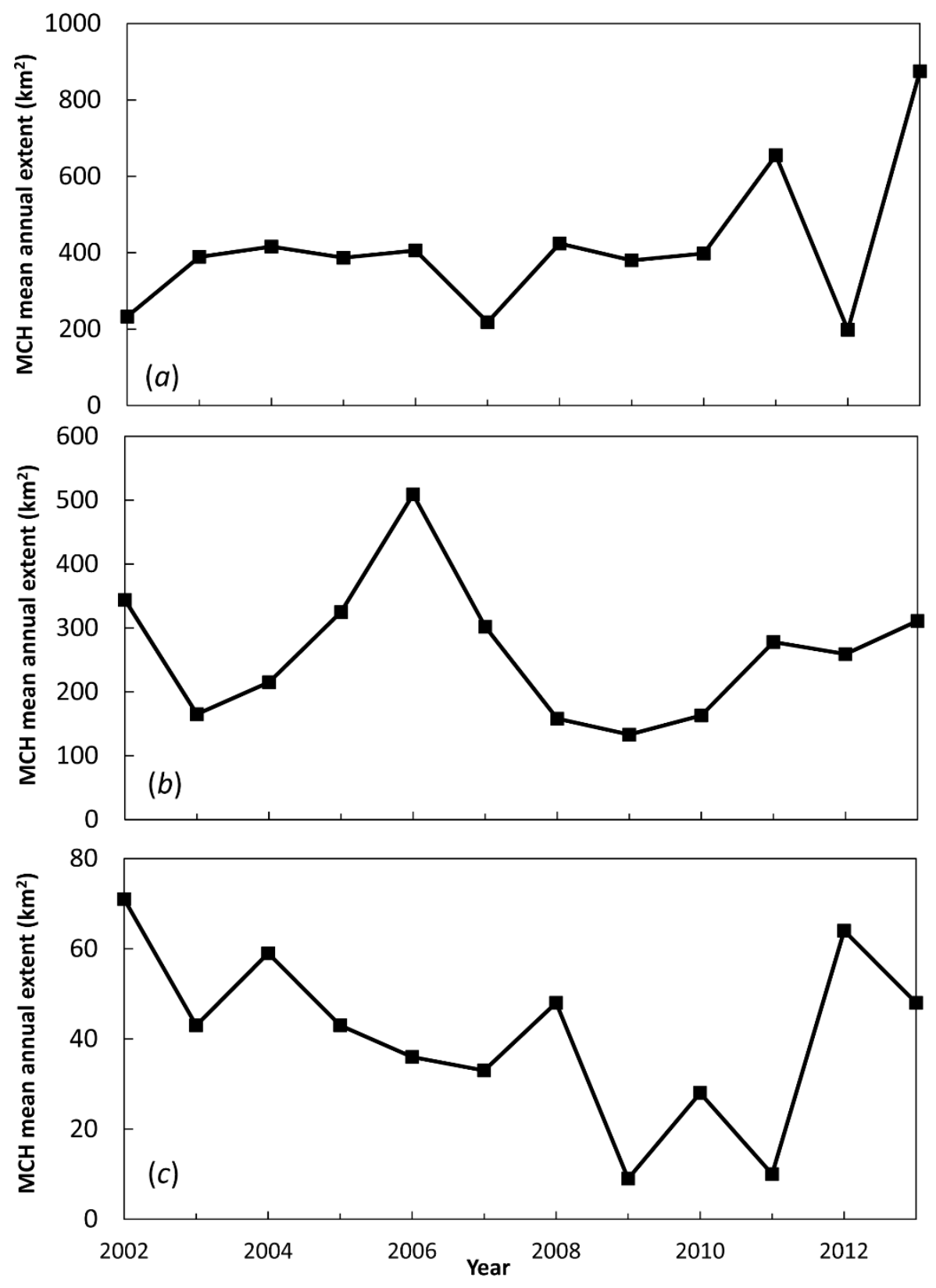

Figure 4.3. $\mathrm{MCH}$ derived mean annual cyanoHAB extent for $(a)$ WBLE, $(b)$ $\mathrm{SB}$, and (c) GB from 2002-2013. 

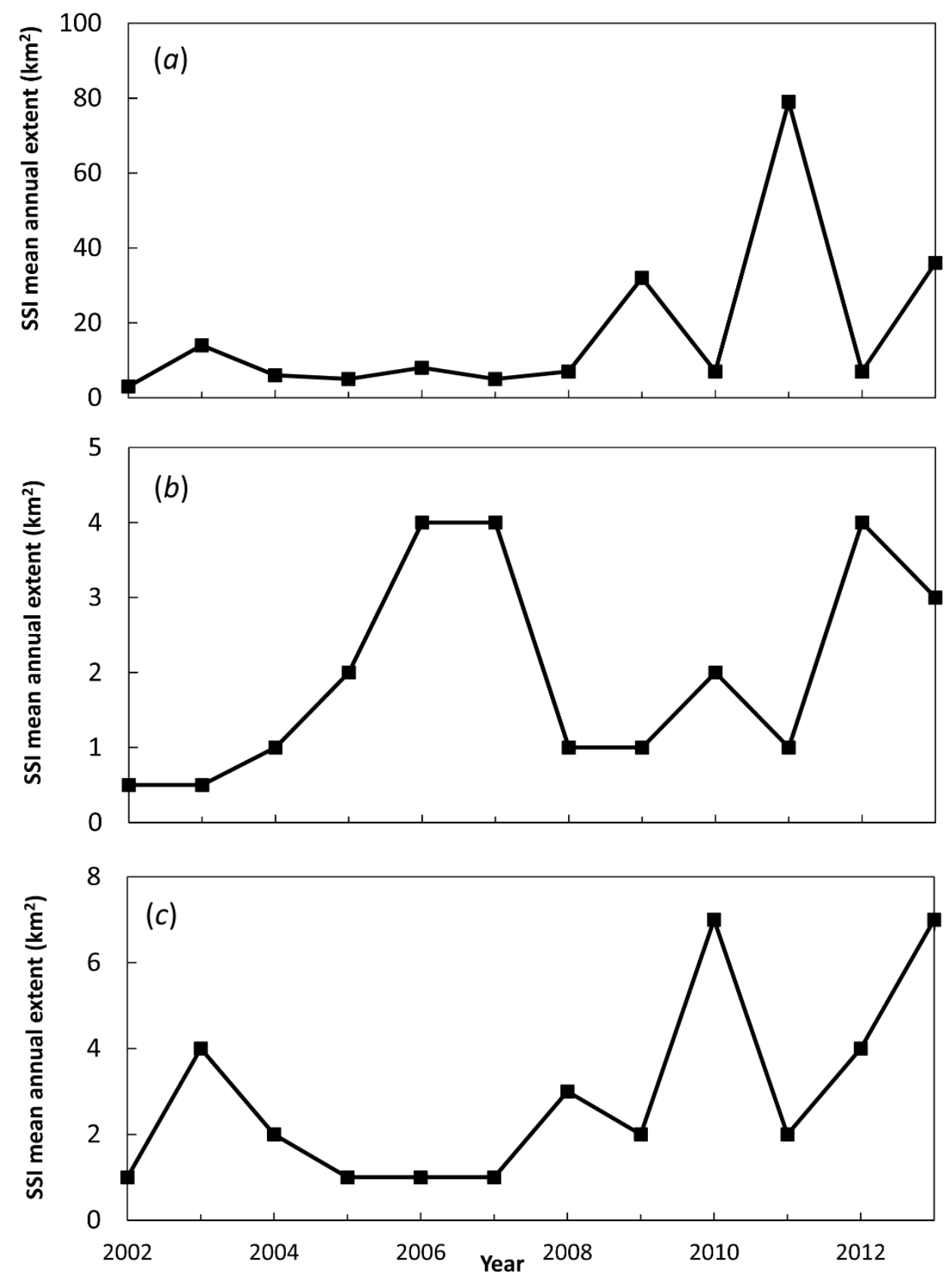

Figure 4.4. SSI derived mean annual surface scum extent for $(a)$ WBLE, $(b)$ SB, and (c) GB from 2002-2013. 
Significant temporal and spatial variability in cyanoHAB occurrences were observed within and among basins. Cyano HAB occurrences range from 0-28 days in WBLE and $\mathrm{SB}$, and 0-14 days in GB (Figures 4.5-4.7). In the WBLE, cyanoHAB events most often occur in the southwestern area near the mouth of the Maumee River. Long lasting cyanoHAB events were also observed in 2011 and 2013 around the islands to the west. Very few cyanoHAB events were observed near the mouth of the Detroit River in the north, with the exception of 2011. CyanoHAB events in SB occurred most frequently in the southeastern portion of the basin away from the mouth of the Saginaw River. Similar to WBLE, cyanoHABs in GB were most often observed near a dominant river mouth, the Fox River, however the persistence was low ( $<7$ days).

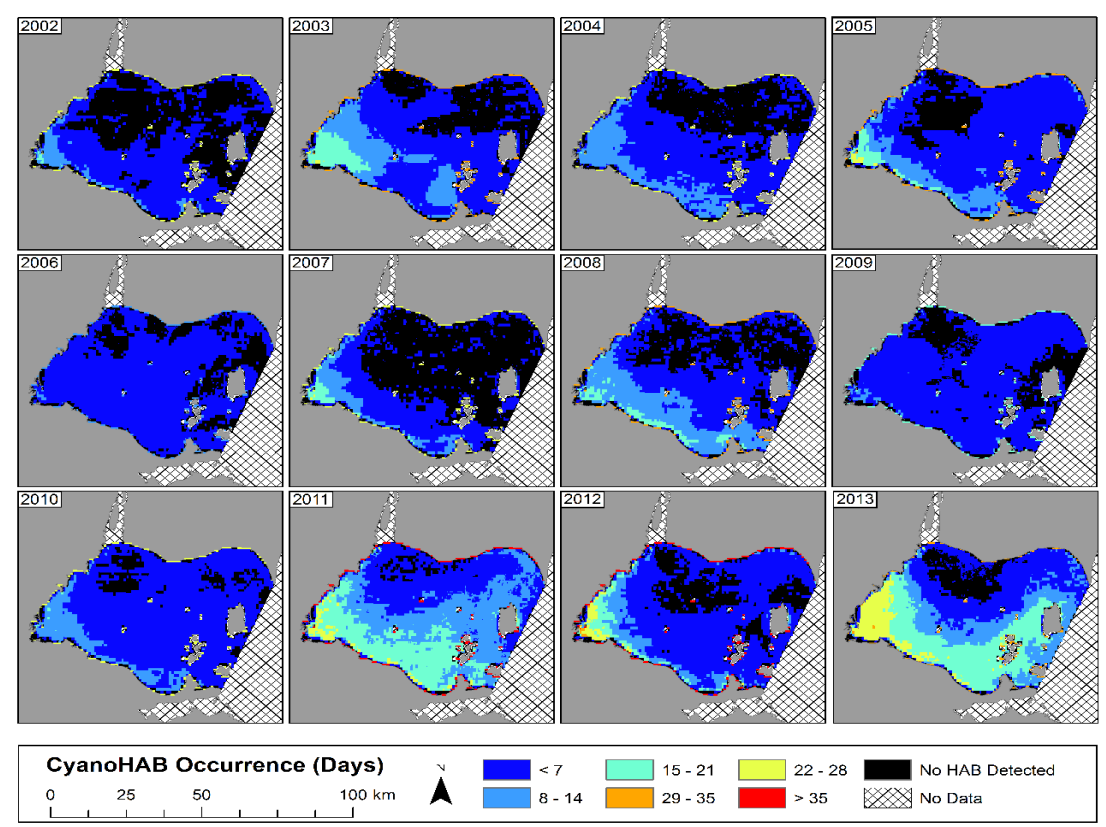

Figure 4.5. CyanoHAB occurrence maps for WBLE from 2002-2013. Colored areas indicate the number of days cyanoHABs were present while areas in black experienced no cyanoHAB presence. 


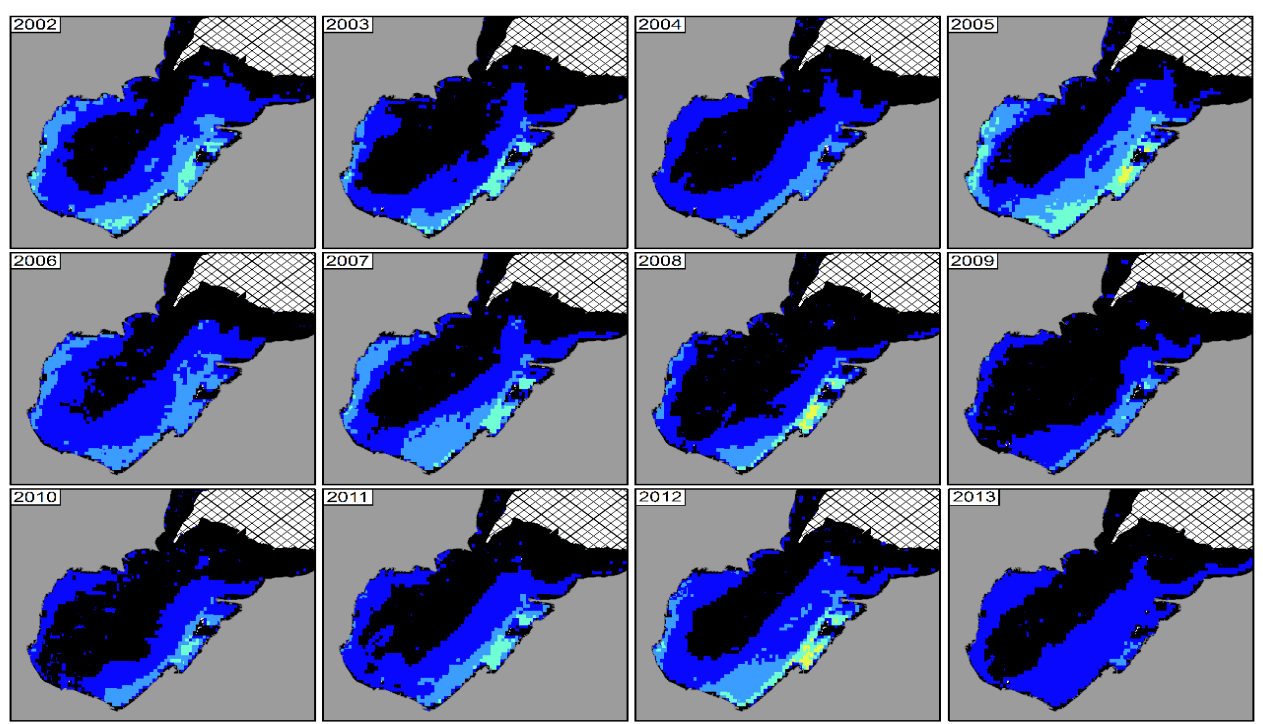

CyanoHAB Occurrence (Days)

Figure 4.6. CyanoHAB occurrence heat maps for SB from 2002-2013. Colored areas indicate the number of days cyanoHABs were present areas in black experienced no cyanoHAB presence. 


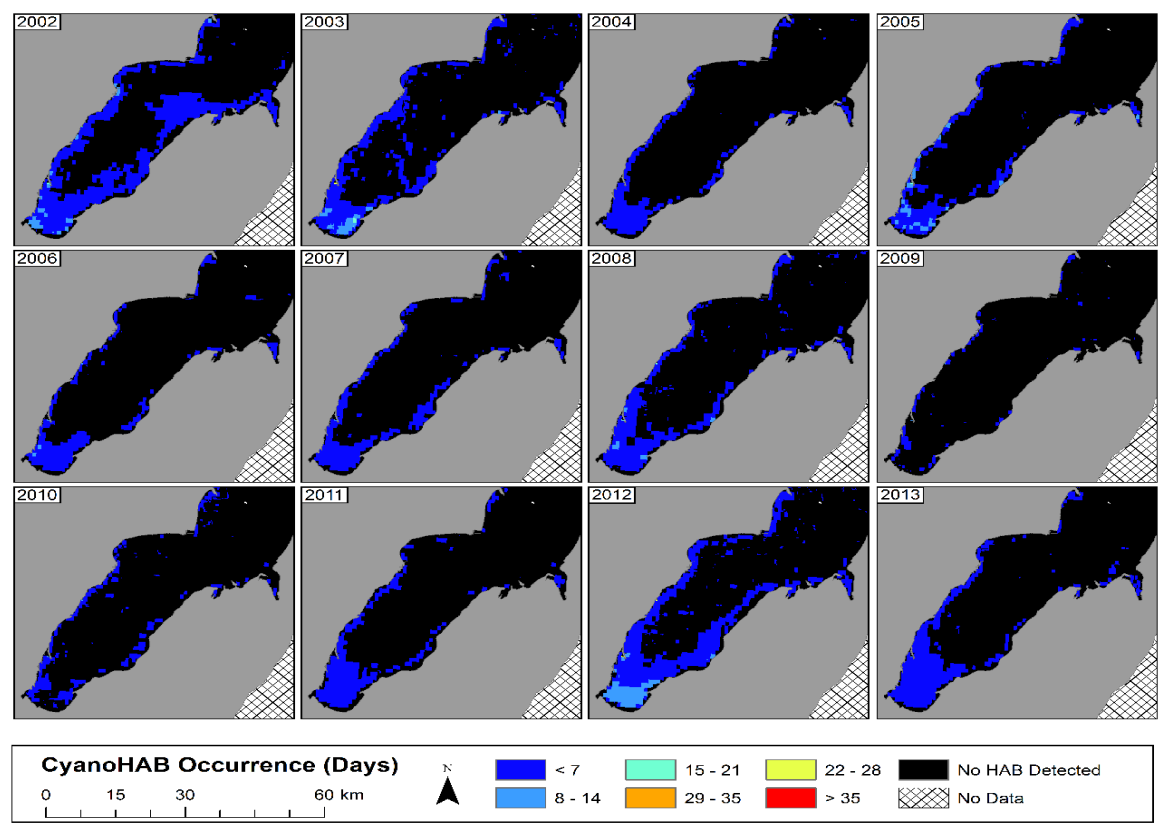

Figure 4.7. CyanoHAB occurrence heat maps for GB from 2002-2013. Colored areas indicate the number of days cyanoHABs were present while areas in black experienced no cyanoHAB presence.

Annual mean air temperature (Celsius $-{ }^{\circ} \mathrm{C}$ ) trends among the three basins were very similar for the 2002-2013 period (Figure 4.8). While the trends among basins were highly correlated (all $r>0.75$, all $p<0.05$ ), there were significant differences in mean values from 2002-2013 $\left(p<0.05\right.$; WBLE $=10.5{ }^{\circ} \mathrm{C}$, Green Bay $=7.7{ }^{\circ} \mathrm{C}$, Saginaw Bay $\left.=8.8{ }^{\circ} \mathrm{C}\right)$. Annual mean air temperature explained more variance in cyanoHAB extent for WBLE than both mean and maximum summer (June-August) air temperature (mean annual $R^{2}=$ $0.22, p=0.12$; mean summer $R^{2}=0.04, \mathrm{p}=0.53$; maximum summer $R^{2}=0.04, p=0.56$ ). There was little explanatory difference between the three air temperature variables in SB (mean annual $R^{2}=0.12, p=0.28$; mean summer $R^{2}=0.13, p=0.24$; maximum summer $R^{2}$ $=0.02, p=0.67$ ) and GB (mean annual $R^{2}=0.03, p=0.57$; mean summer $R^{2}=0.02, p=$ 0.66; maximum summer $\left.R^{2}=0.05, \mathrm{p}=0.50\right)$. 


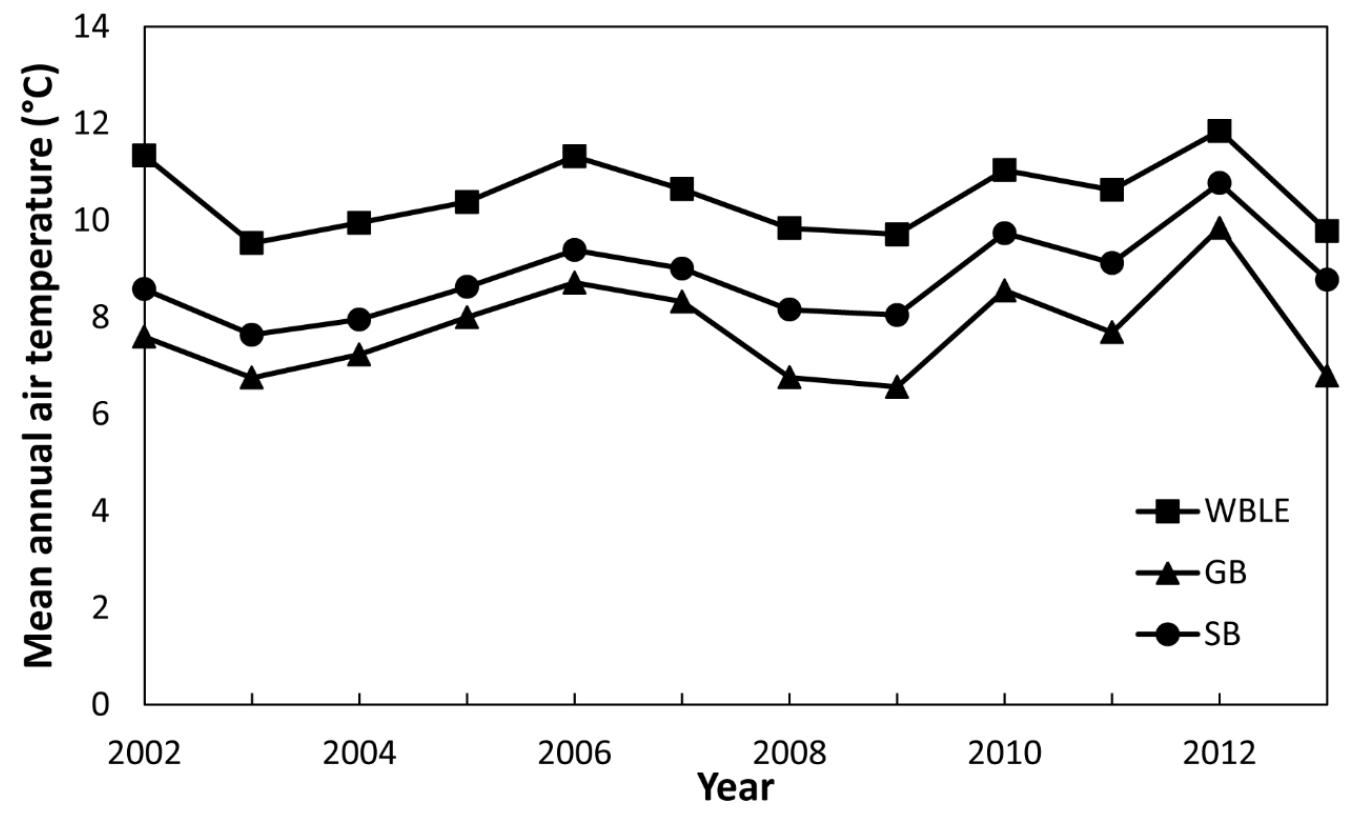

Figure 4.8. Mean annual air temperature for WBLE, GB, and SB from 20022013.

Mean annual precipitation trends varied among basins for 2002-2013 (Figure 4.9). Mean precipitation trends for WBLE correlated significantly with SB $(r=0.52, p<0.05)$, but not GB $(r=0.25, p>0.05)$. There was no significant correlation between trends in mean values for GB and SB ( $p>0.05)$. For mean values during the study period (2002-2013) the only significant difference was noted between WBLE and SB $(p<0.05)(\mathrm{WBLE}=2.5 \mathrm{~mm}$, $\mathrm{SB}=1.8 \mathrm{~mm}, \mathrm{~GB}=2.2 \mathrm{~mm}$ ). 


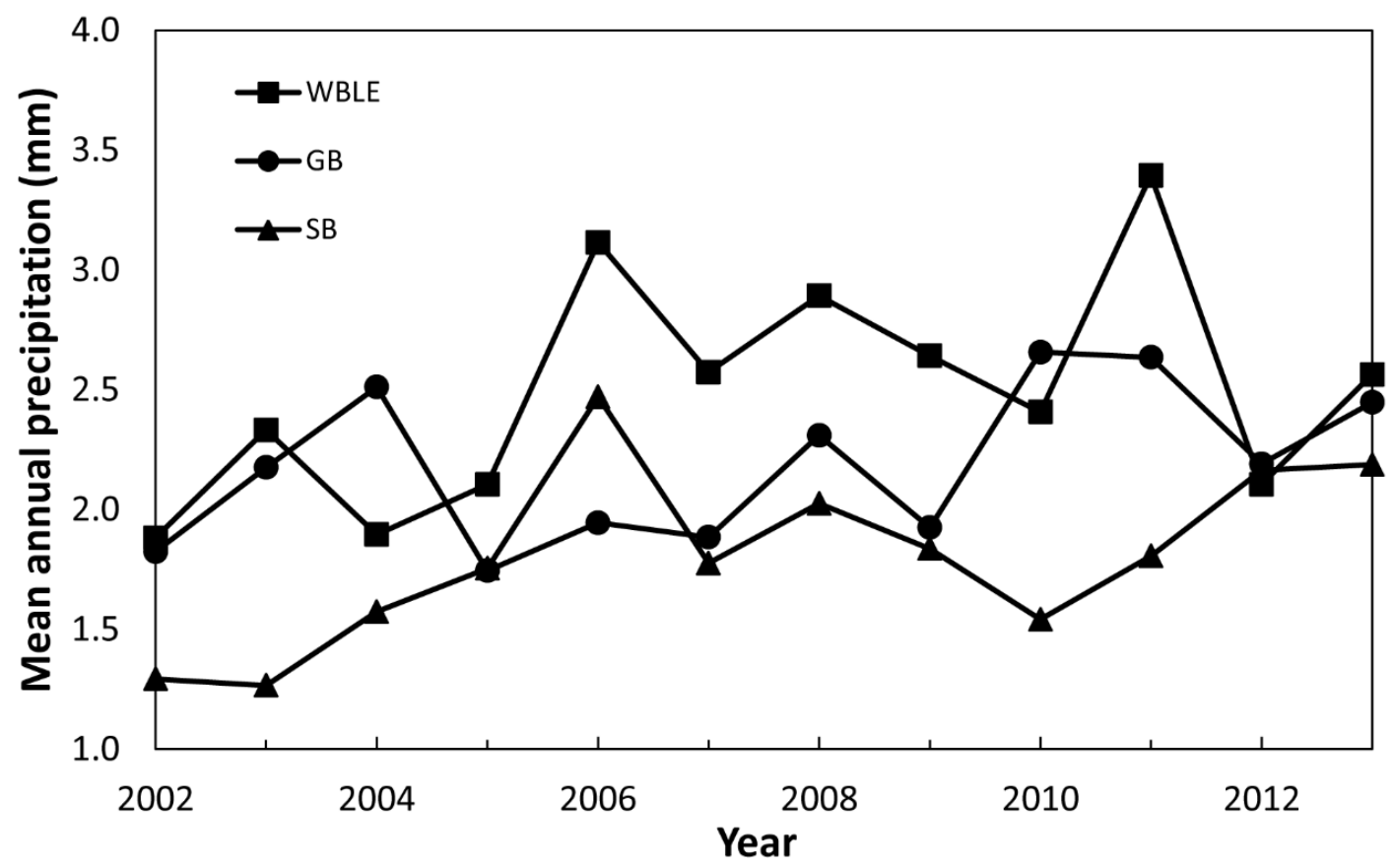

Figure 4.9. Mean annual precipitation for WBLE, GB, and SB from 2002-2013.

Mean annual and spring (March-June) discharge, Q, trends for the Maumee, Fox, and Saginaw Rivers were also compared from 2002-2013 (Figure $4.10(a)$ and $(b)$ ). There were no significant correlations (all $r<0.40$, all $p>0.05$ ) for mean $\mathrm{Q}$, both annual and spring periods (March-June), among any of the three rivers. There were no significant differences in overall mean spring $Q$ among the rivers (Maumee River $=261 \mathrm{~m}^{3} \mathrm{~s}^{-1}$, Fox River $=186$ $\mathrm{m}^{3} \mathrm{~s}^{-1}$, Saginaw River $=224 \mathrm{~m}^{3} \mathrm{~s}^{-1}$; all $p>0.05$ ), however, the Maumee River annual mean $Q$ value $\left(184 \mathrm{~m}^{3} \mathrm{~s}^{-1}\right)$ was significantly different from both the Fox River $\left(123 \mathrm{~m}^{3} \mathrm{~s}^{-1}\right)$, and Saginaw River $\left(134 \mathrm{~m}^{3} \mathrm{~s}^{-1}\right)$ values $(p<0.05)$. 

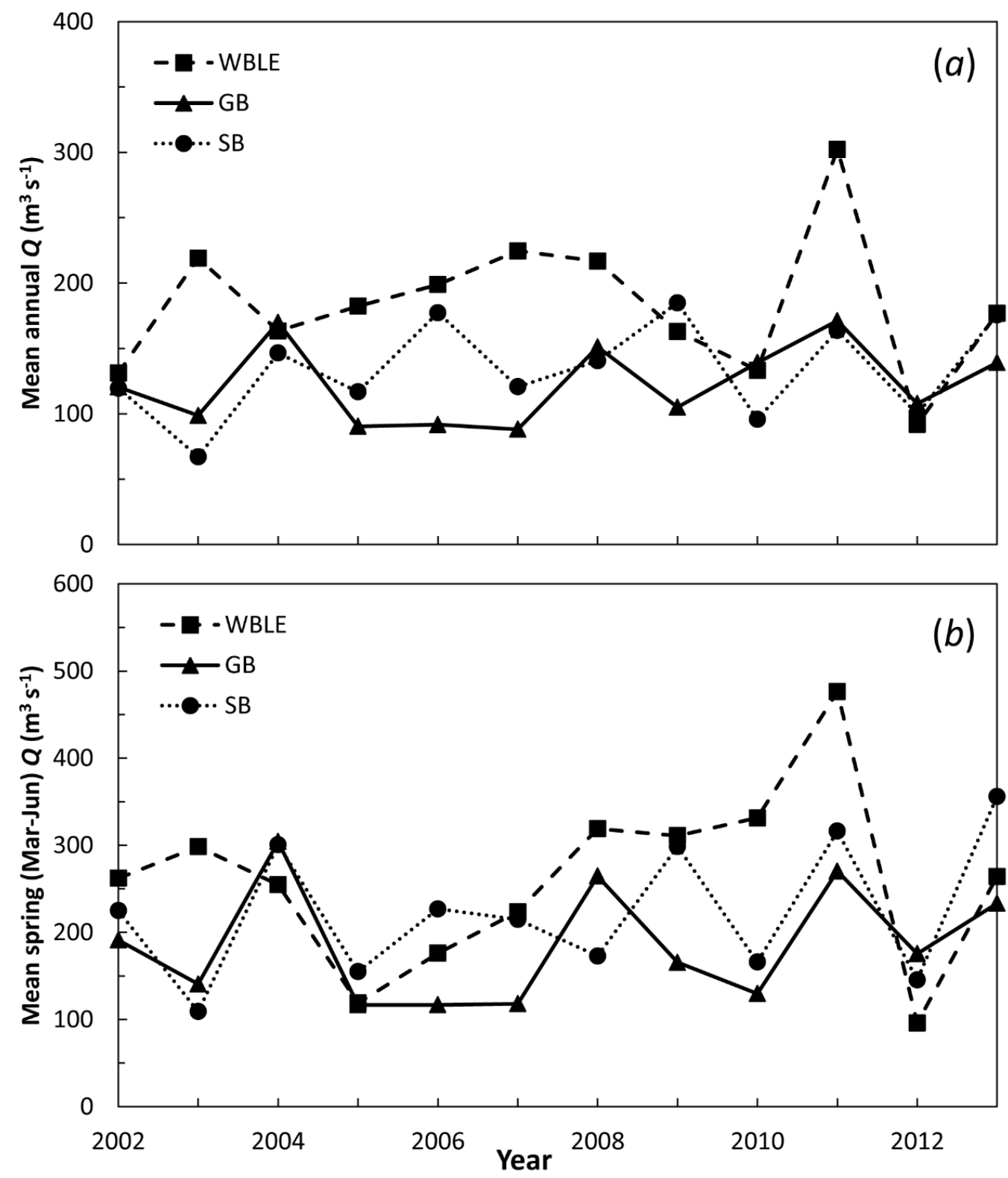

Figure 4.10. ( $a$ ) Mean annual river discharge, $Q$, for WBLE (Maumee River), GB (Fox River), and SB (Saginaw River). (b) Mean spring (March-June) river discharge, $Q$, for WBLE (Maumee River), GB (Fox River), and SB (Saginaw River). Note the y-axis scale ranges are different.

Mean spring and annual $Q$ for the Maumee, Fox, and Saginaw Rivers were compared to both mean MCH and SSI cyanoHAB values for WBLE, GB, and SB from 2002-2013. Only 
for WBLE were significant relationships developed for $Q$ values and cyanoHABs measures (Cubic for SSI, Linear for $\mathrm{MCH}$ ), and better predictors were developed for spring than for annual $Q$ values. For spring $Q$ values and annual SSI, a significant cubic (Figure $4.11(a)$ ) relationship was observed $\left(y=3.29 \mathrm{e}-06 x^{3}-0.002 x^{2}+0.42 x-20.5, R^{2}=0.77, p<0.05\right)$. There was no significant linear relationship between mean spring $Q$ and $\mathrm{MCH}$ values for the Maumee River from 2002-2013, however, the relationship became significant (Figure $4.11(b), y=0.84 x+155, R^{2}=0.50, p<0.05$ ) when 2013 was removed from the data set as a statistical outlier defined as greater than two standard deviations $\left(\sigma=188 \mathrm{~km}^{2}\right)$ from the mean $\left(415 \mathrm{~km}^{2}\right)$. For SB and GB, no significant relationships were observed, linear or cubic, between spring $Q$ and mean annual MCH or SSI values from 2002-2013 (Figures $4.11(c)$ - $(f)$ ). For annual $Q$ and annual HABs (MCH and SSI), there were no significant relationships between mean annual $Q$ and $\mathrm{MCH}$ values for any of the three rivers, however, the relationship between the Maumee River and WBLE MCH values again became significant $\left(y=1.6 x+88, R^{2}=0.49, p<0.05\right)$ when 2013 was removed from the data set. There was also a significant cubic relationship observed between Maumee River annual $Q$ and SSI values $\left(y=3.82 \mathrm{e}-05 x^{3}-0.02 x^{2}+3.34 x-166.4, R^{2}=0.76, p<0.05\right)$. There were no significant relationships, linear or cubic, between annual $Q$ and annual MCH or SSI values for GB or SB from 2002-2013 (all $p>0.05$ ). 

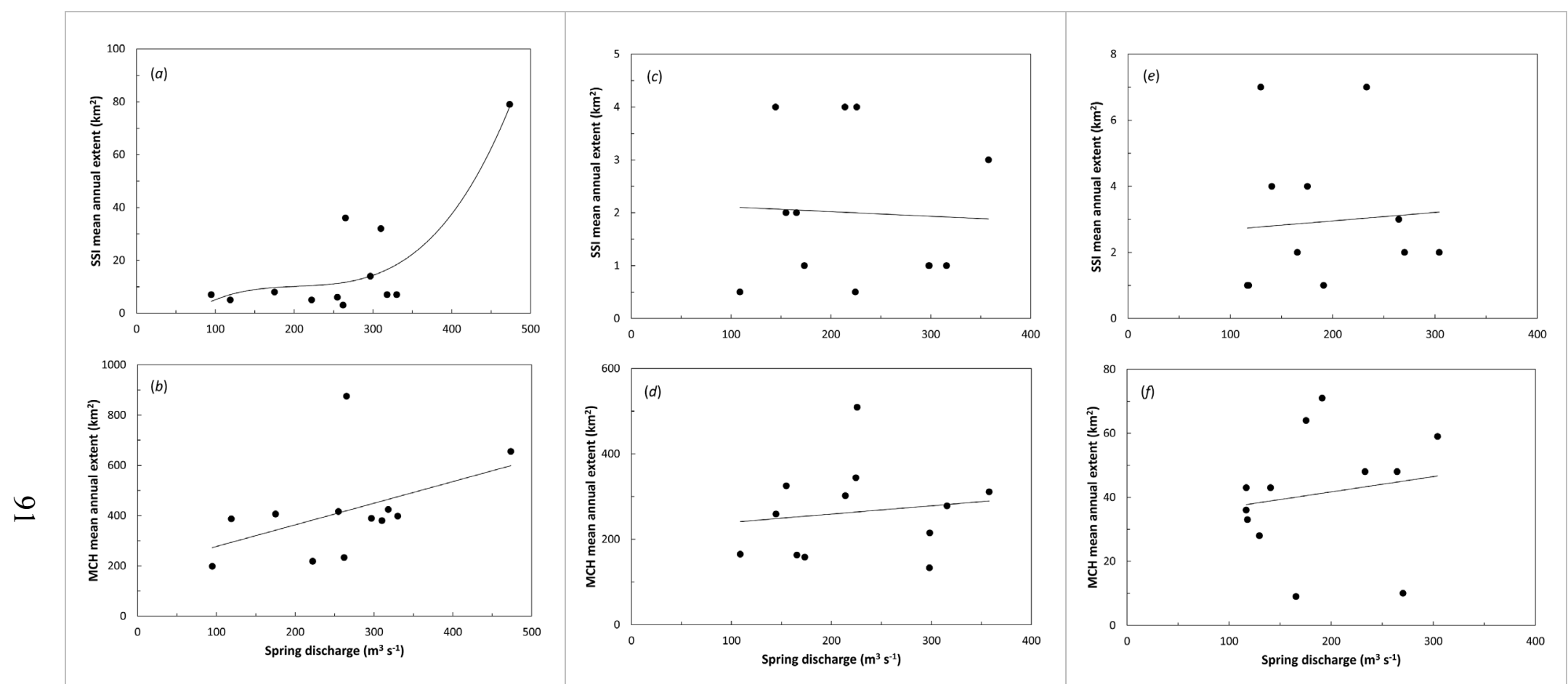

Figure 4.11. (a) SSI mean annual extent vs. spring discharge for WBLE $\left(y=0.0008 x^{2}-0.2844 x+29.226, R^{2}=0.75, p<\right.$ 0.05). (b) MCH mean annual extent vs. spring discharge for WBLE with 2013 removed as a statistical outlier $(y=0.84 x+$ $\left.155, R^{2}=0.50, p<0.05\right)$. (c) SSI mean annual extent vs. spring discharge for SB $(p>0.05)$. (d) MCH mean annual extent vs. spring discharge for SB $(p>0.05)$. (e) SSI mean annual extent vs. spring discharge for GB $(p>0.05)$. (f) MCH mean annual extent vs. spring discharge for GB $(p>0.05)$. 
Strong wind events are possible meteorological drivers for cyanoHAB occurrence in the Great Lakes (Millie et al. 2009; Michalak 2013). Wind induced sediment resuspension events were summed for the bloom period (July-September) in WBLE, GB and SB for 2002-2013. The number of bloom period sediment resuspension events varied considerably in WBLE, with a maximum of 11 in 2011 and a minimum of 0 in 2007 (Figure 4.12). During the study period (2002-2013) there were no strong wind induced resuspension events in Green Bay and only 1 in Saginaw Bay in 2012. These notable differences among regions are probably the result of bloom station location and not meteorological differences among basins.

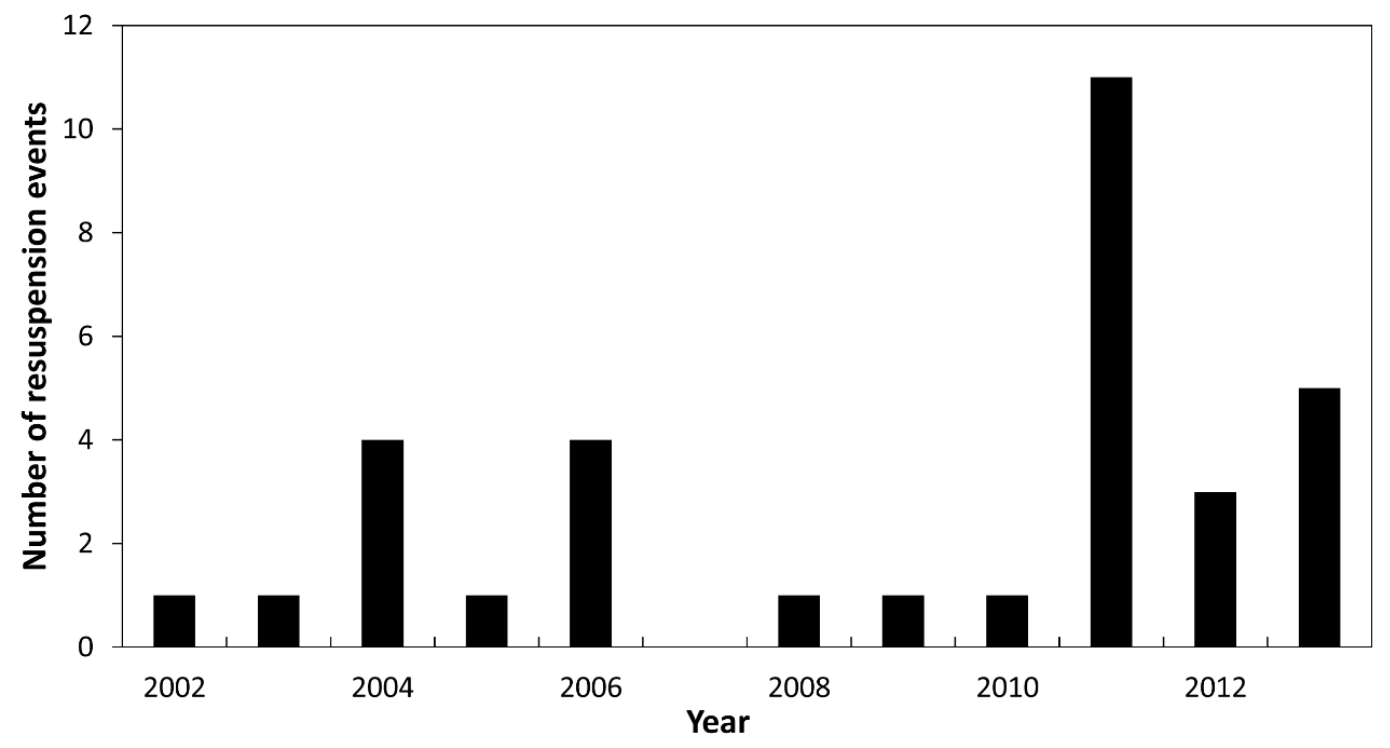

Figure 4.12. The number of wave-induced sediment resuspension event occurrence in the cyanoHAB bloom period (July-September) for WBLE from 2002-2013.

In WBLE wind induced sediment resuspension events during the bloom period (JulySeptember) were compared to mean annual $\mathrm{MCH}$ and SSI values. Significant relationships were identified for both $\mathrm{MCH}$ (Figure $4.13(a), y=39 x+308, R^{2}=0.40, p<0.05$ ) and SSI 
values (Figure $4.13(b), y=6.1 x+0.76, R^{2}=0.69, p<0.05$ ). The points at the high end of the regression lines in Figure $4.13(a)$ and $13(b)$ correspond to the 2011 cyanoHAB event.
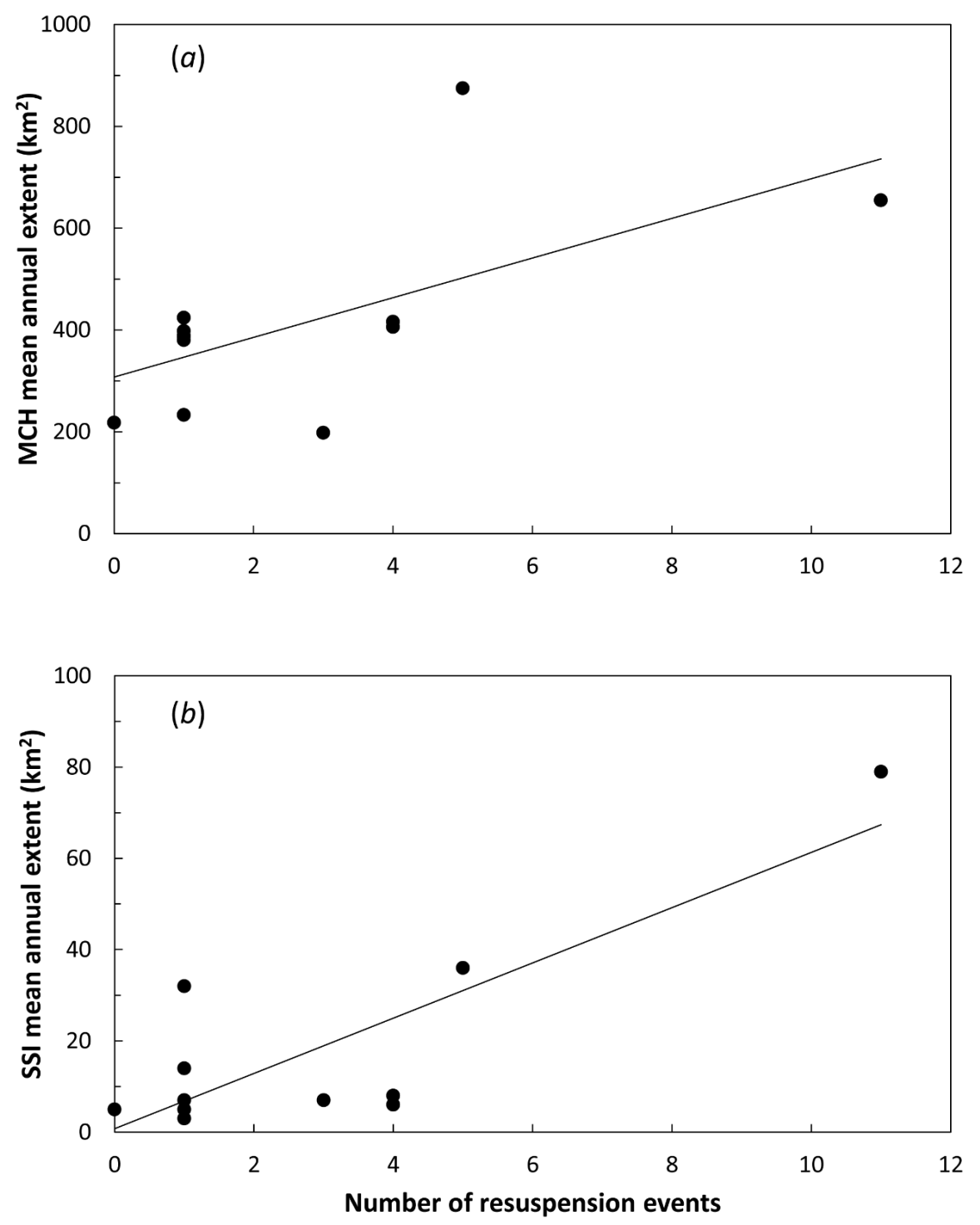

Figure 4.13. The number of sediment resuspension events in the bloom period (JulySeptember) from 2002-2013 for WBLE vs. (a) MCH derived mean annual cyanoHAB extent $\left(y=39 x+308, R^{2}=0.40, p<0.05\right)$. (b) SSI derived mean annual surface scum extent $\left(y=6.1 x+0.76, R^{2}=0.69, p<0.05\right)$. 


\subsection{Discussion}

The usefulness of the new MCH and SSI approaches for estimating water column and surface scum cyanoHAB extents in the Great Lakes has been demonstrated in this study. These two new products augment the HABs mapping product (i.e. Cyanobacteria Index) developed for western Lake Erie (Wynne et al. 2008, 2015; Stumpf et al. 2012). Our new cyanoHAB extent products produced annual trends that are similar to those achieved by prior investigators using the MERIS sensor which has bands sufficient for detecting phycocyanin directly. For example, both the $\mathrm{MCH}$ and SSI mean annual extent trends compared well with estimates reported by Stumpf et al. (2012) for the 2002-2011 period $\left(\mathrm{MCH} R^{2}=0.66, p<0.05 ; \mathrm{SSI} R^{2}=0.91, p<0.05\right)$. The WBLE cyanoHAB occurrence maps (Figure 4.5) generated in this study also show similar patterns as those presented by Wynne et al. (2015) where the most persistent and intense blooms tend to be located in Maumee Bay and along the southern shore of WBLE. The maps presented by Wynne et al. (2015) also show very little cyanoHAB occurrence in the Detroit River outflow in the northern end of WBLE. Moreover, this study reports for the first time satellite-derived cyanoHAB estimates in Green Bay and Saginaw Bay. Our two new approaches have three advantages over those that rely on phycocyanin detection bands; 1) $\mathrm{MCH}$ uses a multispectral bio-optical model to deconvolve observed spectra into water constituent concentrations, thus eliminating the need for pure cyanobacteria spectra, 2) it can be applied to other sensors with sufficient spectral specifications to produce accurate chlorophyll $a$ concentrations to extend time series continuity, and finally 3 ) it expressly delineates surface scum accumulations which often indicate areas of public health concern (i.e. very high toxin content). These new approaches have provided several new insights into the role of regional and local control on cyanoHAB occurrence in the Great Lakes.

Mean annual HAB extent trends among WBLE, Green Bay, and Saginaw Bay from 20022013 varied among the study period, and in particular, demonstrated a departure in recent years. Both mean annual MCH and SSI extents were significantly different among basins with extensive and persistent HAB events in 2011 and 2013 in WBLE whereas GB and SB 
experienced mild events in those years. Thus, the recent trend of increasing HABs in WBLE noted by Michalak et al. (2013) was not found in SB or GB. Furthermore, there were drastic differences in cyanoHAB persistence among basins with parts of WBLE experiencing cyanoHAB conditions for more than 30 days in recent years (2011-2013) while GB and SB are under those conditions for less than 15 days. These dissimilarities in extent and duration indicate either substantial annual differences in controlling factors among basins and/or locally varying relationships between these controlling factors and cyanoHAB occurrence.

The recent increase in cyanoHABs in WBLE has been attributed to increased nutrient loading (local control) and climate change (regional) (Michalak et al. 2013). Because no increase in cyanoHABs was noted in either SB or GB in recent years, it is likely that dissimilarities in these environments are likely more important in controlling the annual cycle of cyanoHABs than regional climate. Climate change may increase the frequency of cyanoHABs within all eutrophic basins in the Great Lakes, but controlling local factors (nutrients, invasive species, etc.) need more attention for mitigation of cyanoHABs.

Even though these three shallow, eutrophic basins in the Great Lakes appear to be similar, they have important differences. For example, the basins vary tremendously in morphometry (i.e. mean depth, retention time, etc.), land use, and latitude (i.e. meteorology) within each basin. Water depth characteristics for the three basins are considerably different, undoubtedly affecting hydrodynamic processes including water column mixing and sediment re-suspension (WBLE mean $\sim 7.5 \mathrm{~m}$, maximum $<20 \mathrm{~m}$, GB mean $16 \mathrm{~m}$, maximum $>30 \mathrm{~m}$, SB mean $\sim 4.5 \mathrm{~m}$ inner bay and $15 \mathrm{~m}$ outer bay, maximum $>40 \mathrm{~m}$ ). The hydraulic retention time of each basin also varies (WBLE $\sim 35$ days, SB $\sim 60$ days, GB $\sim 290$ days) considerably, thus affecting the dispersion rates of phosphorus and other nutrients delivered from river discharge. Agricultural land use, through fertilization practices, is the primary upland source of nutrients into the dominant rivers, and varies among basins. For example, agricultural land use in the Maumee River watershed is almost two-fold greater than both the Saginaw and Fox River watersheds (Maumee River $\sim 80 \%$, 
Saginaw River $\sim 48 \%$, Green Bay $\sim 44 \%$ ). Locational differences of the basins with respect to latitude also play a key role in meteorological dissimilarities. For example, significant differences in the mean annual air temperature were observed between basins, where GB was cooler than both SB and WBLE which was probably related to latitudinal variation.

These basin differences contribute to the dominant role that local factors play in controlling the annual trend in cyanoHABs even though nutrient loading in all three basins is dominated by 1-2 rivers. The role of these major rivers in controlling annual HABs varies as evident by the relationships between river discharge and cyanoHAB extent. Only for WBLE were significant relationships developed between spring (March-June) river discharge, Q, of the Maumee River and both SSI (cubic) and MCH extent (linear), excluding 2013. No significant relationship was observed between spring $Q$ and cyanoHAB occurrence for either GB (Fox River) or SB (Saginaw River). Similarly, several spring monthly discharge rates (March-May) exhibited significant relationships with cyanoHAB extents in WBLE. In GB and SB, only a few monthly discharge rates were correlated with cyanoHABs and most were from summer and fall (June-September). The stronger relationship between discharge of the Maumee and WBLE cyanoHABs might be surprising given the Saginaw River and Fox River contributes almost $90 \%$ and $70 \%$ of the loading to Saginaw Bay and Green Bay, respectively whereas the Maumee River contributes only $40 \%$ of nutrient loading to the WBLE.

Our relationships between spring $Q$ and cyanoHABs in WBLE are similar to those relationships noted by other investigators. Stumpf et al. (2012), using MERIS data for 2002-2011, developed a predictive model between spring $Q$ and cyanoHABs in WBLE. The cubic relationship established between SSI and spring $Q$ for WBLE was expected as Stumpf et al. (2012) observed a similar relationship between spring $Q$ and their annual Cyanobacteria Index (CI) concentrations. Both of these relationships are heavily influenced by the 2011 cyanoHAB occurrence which has been documented as the most severe on record (Bridgeman et al. 2013, Michalak et al. 2013). Obenour et al. (2014) proposed a Bayesian model linking cyanoHAB size to total phosphorus loading from the 
Maumee River. Obenour et al. (2015) also note that WBLE is becoming increasingly susceptible to extensive blooms, independent of loading, suggesting other environmental processes may ultimately be controlling bloom formation. These other potential controlling processes may be responsible for the observed large $\mathrm{MCH}$ extent in 2013 while Maumee spring $Q$ for that year $\left(261 \mathrm{~m}^{3} \mathrm{~s}^{-1}\right)$ was very similar to the 2002-2013 mean Maumee $Q\left(265 \mathrm{~m}^{3} \mathrm{~s}^{-1}\right)$. Presently, there has been no attempt to apply these approaches to the other eutrophic basins in the Great Lakes where cyanoHAB blooms occur. The results of this study demonstrate the apparent inability of spring $Q$ to predict cyanoHAB extent in SB or GB suggesting that other local factors (basin morphometry, physical mixing and forcing, delivery of nutrients, local meteorology, etc.), influence the development of cyanoHABs.

The importance of local factors in controlling HABs in these basins extends to meteorological forcing such as resuspension or complete water column mixing. Despite the general similarity of basins, only one of these regions (WBLE) had a large number of sediment resuspension events during the bloom period. Moreover, the number of resuspension events was related to cyanoHAB extent in WBLE. In SB and GB very few events were noted most likely due to the location of the bloom areas within each basin and basin morphometry. Previous investigators have noted the possibility that sediment resuspension is an important seeding mechanism for cyanobacteria blooms. Bottom sediment reservoirs in WBLE have been shown to contain cyanobacteria cells that when released back into the water column through wave induced resuspension, can contribute to bloom formation (Rinta-Kanto et al. 2009). Michalak et al. (2013) hypothesized for the 2011 WBLE bloom strong winds and associated resuspension events immediately preceding bloom onset contributed to rapid initial bloom formation, however, it was also suggested that a higher frequency of quiescent conditions after bloom onset was more conducive to bloom growth (Millie et al. 2009). The exact relationship between cyanoHAB events and meteorological forcing in these three basins needs to be examined with greater temporal (days-weeks) and spatial resolution (1 km to tens of kilometres). The results of this study have demonstrated for WBLE that more frequent re-suspension events 
in the bloom period (July-September) are moderately associated with larger annual HAB extents.

The local differences among cyanoHAB occurrences in these three basins extend to physical/biological factors as well. Large-scale scums (high SSI values) were noted in WBLE but not in SB or GB despite the presence of large cyanoHABs in all three environments. For example, WBLE produced large mats $\left(>30 \mathrm{~km}^{2}\right)$ of surface scum in 2011 and 2013 whereas GB and SB produced limited scum $\left(<8 \mathrm{~km}^{2}\right)$ for the study period. Scums in WBLE accounted for up to $10 \%$ of the total HAB extent but in GB and SB they account for less than $1 \%$. These observed differences in scum occurrence are not unique to the Great Lakes as cyanoHAB surface scums have been shown to vary considerably in different water bodies throughout the world (Jupp et al. 1994; Quibell 1992; Galat and Verdin 1989). Some evidence suggests cyanoHAB scum formation is a mechanism to achieve dominance over other phytoplankton communities, and is regulated by biological and physical parameters (Paerl and Ustach 1982). However, it is not clear whether physical (calm periods, etc.) or biological (physiological difference associated with clonal variations, etc.) factors are dominant in contributing to the variability among scums in these three Great Lakes basins.

One of the most important contributions of this study is the refinement and application of an index that can classify the presence of surface scum accumulations of cyanobacteria in the Great Lakes, and likely many other environments. Cyanobacteria often form surface scums (Zohary and Robarts 1990; Paerl and Ustach, 1982; Sellner, 1997) where extremely high cell densities are found. High toxin concentrations are often associated with these scums, and health organizations have used the presence of scums as an important health signal (Bartram and Rees 1999). Because of this link between scums and possible high concentrations of toxins, our approach is not only useful for tracking HABs but may also serve as an initial public health alert. Moreover, because this approach does not depend on local empirical relationship like $\mathrm{MCH}$ or any specific calibration, and is defined directly for apparent optical properties, it could likely be used as a generic scum classification 
index. Thus, it is likely that we have developed a simple, generic classification approach capable of providing useful public health information on cyanoHABs from remote sensing platforms.

\subsection{Acknowledgements}

This work was supported by the U.S. Environmental Protection Agency under grant GL-00E008550, the Great Lake Observing System under contract \# 3002475304, and The University of Michigan Water Center under contract\# 3003032930. The authors express their appreciation to Tom Johengen and the Cooperative Institute for Limnology and Ecosystems Research (CILER) for providing in situ data, Zach Raymer, Amanda Grimm, and Karl Bosse for their assistance in data collection and processing. We would like to thank three anonymous reviewers for their very insightful comments. 


\section{Satellite Monitoring of Harmful Algal Blooms in the Western Basin of Lake Erie: a 20-Year Time-Series}

Blooms of harmful cyanobacteria (cyanoHABs) have occurred on an annual basis in western Lake Erie, for more than a decade. Previously, we developed and validated an algorithm to map the extent of the submerged and surface scum components of cyanoHABs using MODIS ocean-color satellite data. The algorithm maps submerged cyanoHABs by identifying high chlorophyll concentrations $\left(>18 \mathrm{mg} / \mathrm{m}^{3}\right)$ combined with water temperature greater than $20^{\circ} \mathrm{C}$, while cyanoHABs surface scums are mapped using near-infrared reflectance values. Here, we adapted this algorithm for the SeaWiFS sensor to map the annual areal extents of cyanoHABs in the Western Basin of Lake Erie for the 20-year period from 1998 to 2017. The resulting classified maps were validated by comparison with historical in situ measurements exhibiting good agreement ( $81 \%$ accuracy). Trends in the annual mean and maximum total and surface scum extents demonstrated significant positive increases from 1998-2017. There was also an apparent 76\% increase in year-to-year variability of mean annual extent between the 1998-2010 and 2011-2017 periods. The 1998-2017 time-series was also compared with several different river discharge nutrient loading metrics to assess the ability to predict annual cyanoHAB extents. The prediction models displayed significant relationships between spring discharge and cyanoHAB area, however, substantial variance remained unexplained due in part to the presence of very large blooms occurring in 2013 and 2015. This new multi-sensor time-series and associated statistics extend the current understanding of the extent, location, duration, and temporal patterns of cyanoHABs in western Lake Erie.

Keywords: Lake Erie; Harmful algal blooms; Satellite; Cyanobacteria; Time series; Remote sensing 


\subsection{Introduction}

Cyanobacteria blooms are recurring events within select biologically-productive waters of the Great Lakes, notably the Western Basin of Lake Erie (WBLE) (Vanderploeg et al., 2001; Bridgeman et al., 2013). Harmful algal blooms dominated by the cyanobacterium Microcystis (cyanoHABs) have affected recreation, charter fishing, and tourism in the Great Lakes and placed drinking water supplies for millions of consumers at risk. A cyanoHAB in western Lake Erie in 2014 resulted in a three-day tap water ban for approximately half a million customers in Toledo, Ohio (Wynne and Stumpf, 2015). Microcystis produces microcystin, a hepatotoxin that can cause gastroenteritis, liver damage and, in extreme cases, more serious illness and even death (Rinta-Kanto et al., 2005). Microcystis blooms have occurred on an annual basis in the Western Basin of Lake Erie for more than a decade (Budd et al., 2001; Rinta-Kanto et al., 2005; Stumpf et al., 2012; Steffen et al., 2014).

Due to their temporal and spatial variability ("patchiness"), cyanoHABs are extremely difficult to monitor using buoy- or ship-based sampling (Kutser, 2004). Their concentrations can vary by multiple orders of magnitude over the distance of a few meters. Satellite remote sensing provides the potential for long-term synoptic monitoring of cyanoHAB events, which can improve our understanding of these phenomena to allow for the development of approaches that reduce their incidence over the longer term.

Satellite remote sensing has been extensively used to map cyanobacteria bloom dynamics in many areas throughout the world with great success (Pettersson and Pozdnyakov 2013). Several early electro-optical (EO) satellite platforms including Landsat 2 and the Coastal Zone Color Scanner (CZCS) proved useful to detect significant accumulations of cyanobacteria blooms in the Baltic Sea (Ulbricht 1983) and southwestern tropical Pacific Ocean (Dupouy et al. 1988). Additionally, the Advanced Very High Resolution Radiometer (AVHRR) thermal imagery was used to relate local increases in sea surface temperature (SST) to surface accumulations of cyanobacteria in the Baltic Sea (Kahru et al. 1993). Since the publication of these early studies, research using satellite remote 
sensing to map cyanobacteria has expanded in the literature (Cullen et al. 1997) with work including many areas throughout the world, with notable examples in the Baltic Sea (Kahru and Elmgren 1997, Kahru et al. 2000, 2007, 2014, Miller et al. 2006, Reinart and Kutser 2006), Lake Taihu (Duan et al. 2009, 2012, Hu et al. 2010, Huang et al. 2014), and South African Lakes (Matthews et al. 2010, 2012, 2013, 2014, 2015; Oberholster and Botha 2010).

Remote sensing algorithms have progressed from the early radiance threshold approaches to more robust empirical and semi-analytical methods as observing system capabilities have increased and better understanding of the underlying cyanobacteria optical properties were achieved (Mishra et al.2017). Robust algorithms to retrieve chlorophyll-a concentrations of cyanobacteria blooms have been developed and adapted for many different freshwater systems to track spatio-temporal dynamics (Gons 1999, Gons et al. 2008, Mishra et al. 2012, Li et al. 2013. In parallel, algorithms were also developed (Dekker 1993, Schalles and Yacobi 2000, Simis et al, 2005, 2007, Li et al. 2015) to estimate the presence of the phycocyanin, a phycopillipigment unique to cyanobacteria, allowing for better determination of bloom composition. However, while many cyanobacteria remote sensing algorithms have been developed over the past 30 years, regional tuning or parametrization is often required to adapt to the local scale cyanobacteria /phytoplankton dynamics of individual systems.

In some areas within the Great Lakes basin, cyanoHABs have been shown to occur in highly productive waters that are relatively shallow, warm, and protected from persistent offshore winds (Ho and Michalak, 2015; Watson et al., 2016). These productive waters are optically complex with the color of the water being determined by the concentrations of three color-producing agents (CPAs): chlorophyll (CHL), colored dissolved organic matter (CDOM), and suspended mineral (SM) particles (Jerlov, 1976; Bukata et al., 1995). Traditional marine water color retrieval algorithms developed for the open ocean, whose optical properties are dominated by phytoplankton absorption and scattering alone, typically fail in optically complex waters (Budd and Warrington, 2004; Witter et al., 2009; 
Ali et al., 2014). Additionally, traditional marine atmospheric correction procedures often yield erroneous water leaving radiance values in optically complex water further reducing the ability of traditional ocean color retrieval algorithms to produce reliable results (Dash et al. 2012).

Several methods have been developed and adapted to identify Great Lakes cyanoHABs based on algorithms that relate spectral reflectance values in ocean color satellite imagery to in situ water measurements (e.g., Becker et al., 2009; Wynne et al., 2010; Stumpf et al., 2012; Sayers et al., 2016). Of these, the most commonly applied approach has been the cyanobacteria index (CI) developed by Wynne et al. (2008) based on the Fluorescence Line Height (FLH) algorithm (Abbott and Letelier, 1999). Stumpf et al. (2012) used this approach to quantify cyanoHABs in western Lake Erie from 2002 to 2011 and relate the annual bloom extent to river discharge. More recently, Sayers et al. (2016) generated cyanoHAB extent maps using a combination of two modified remote-sensing approaches using the MODIS Aqua sensor (MCH and SSI, see Methods section). Here, we adapt the $\mathrm{MCH}$ and SSI approach to cyanoHAB mapping for the SeaWiFS sensor to extend the available time series of ocean color cyanoHAB estimates for western Lake Erie from 1998 to 2017 .

Because of the limited time frame for which cyanoHAB maps are available from the MODIS Aqua and MERIS ocean color satellites (now 16 years, 2002-2017), the ability to empirically model the various drivers of annual bloom size and spatial distribution has been limited (Obenour et al., 2014). Ho et al. (2017a) recently explored extending the MODIS/MERIS ocean color time series by combining the Wynne/Stumpf CI with a Landsat-based algorithm. They found good coherence between the Landsat product and CI with respect to the macro-scale characteristics of annual peak bloom area. However, Landsat's utility for monitoring the temporal variations of the western Erie cyanoHAB within a season are seriously limited by the satellite's 16-day revisit cycle and by the high annual mean cloud cover in the Great Lakes (Ju and Roy, 2008), resulting in few cloudfree Landsat scenes of western Lake Erie collected per season. The combined 
SeaWiFS/MODIS time series of cyanoHAB extent presented here can both serve as additional years of evaluation data for Landsat algorithms and help improve the knowledge of Lake Erie cyanoHAB spatial heterogeneities and finer-scale temporal variability needed to gain a mechanistic understanding of the dynamics of cyanoHAB development and distribution.

In this study, we investigate the coherence of SeaWiFS and MODIS-based cyanoHAB products in a combined time series of hindcast cyanoHAB extent for western Lake Erie. A series of empirically-based adjustments were made to reconcile the cyanoHAB classification differences between the two platforms observed for overlapping images. The aims of this study are to (i) document the adaptation of the MCH/SSI approach for cyanoHAB mapping to the SeaWiFS satellite; (ii) evaluate the SeaWiFS-derived cyanoHAB maps through comparison with in situ data and with MODIS-derived maps for overlapping dates; and (iii) revisit the Sayers et al. (2016) discussion of the local factors driving annual cyanoHAB dynamics in light of this longer time series.

\subsection{Methods}

\subsubsection{Satellite Imagery}

SeaWiFS and MODIS uncalibrated Level 1A imagery was downloaded from the NASA OceanColor data portal (https://oceancolor.gsfc.nasa.gov). Level 1A data were processed to Level 2 (georeferenced with atmospheric and radiometric calibrations) using NASA SeaDAS software. A fixed model pair aerosol correction and custom cloud masking approach was applied to the images to ensure that the high NIR reflectance values of surface scum pixels were not interpreted as atmospheric contamination (Sayers et al., 2016). Additionally, Sayers et al. (2016) reported that the fixed-model pair aerosol selection approach resulted in more consistent and accurate remote sensing reflectance values than the standard oceanic black pixel assumption method during significant cyanoHAB events in western Lake Erie. 
Each cloud-free or mostly ( $>80 \%$ ) cloud-free image of the Western Basin of Lake Erie (Figure 5.1) collected by SeaWiFS between mid-1997 and 2007 or by MODIS Aqua between mid-2002 and 2017 at times when the water temperature in the Western Basin was above $20^{\circ} \mathrm{C}$ - the minimum temperature favorable to cyanoHAB development (Sayers et al., 2016) — was downloaded. The $20^{\circ} \mathrm{C}$ threshold was developed to reduce the number of false cyanoHAB identifications in cooler water $\left(<20^{\circ} \mathrm{C}\right)$ periods when blooms of other phytoplankton, particularly diatoms, can occur (Sayers et al. 2016). When an image with partial cloud cover or other interference was collected within 3 days of a better-quality image, only the better scene was retained in the image set. The dates when the temperature exceeded and then fell below the $20^{\circ} \mathrm{C}$ threshold for each year were determined using the AVHRR (pre-2002) and MODIS (2002-2017) Sea Surface Temperature (SST) product. The satellite observed water temperatures were verified with buoy measurements from the National Data Buoy Center (NDBC) when available (https://www.ndbc.noaa.gov/). 


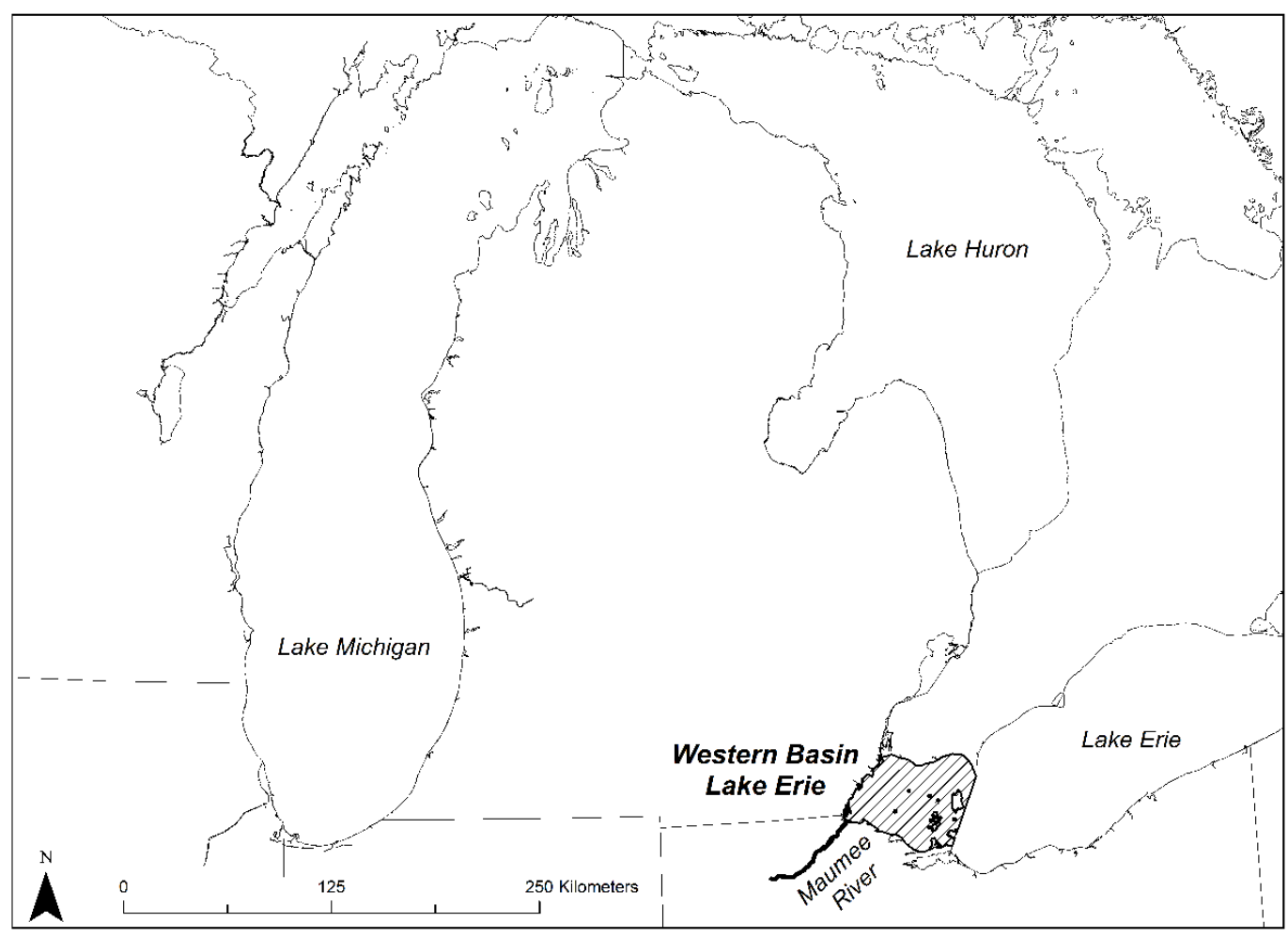

Figure 5.1: The western Lake Erie study area is defined by the hatched area. The Maumee River is also shown as bold black line entering the basin.

\subsubsection{Bio-optical Models}

The hybrid bio-optical and vegetation index method of cyanoHAB mapping described in Sayers et al. (2016) was applied to MODIS Aqua imagery from 2014 through 2017 to extend the 2002-2013 time series presented in that paper. Briefly, the first approach (modified CPA-A HABs, or MCH) was a modification of the CPA Algorithm (CPA-A), a semi-analytical bio-optical chlorophyll retrieval algorithm which simultaneously optimizes estimated concentrations for all three CPAs using a hydro-optical model derived from extensive in situ measurements collected in western Lake Erie (Shuchman et al., 2006; 2013). For cyanoHAB detection, the CPA-A is enhanced with empirical relationships between chlorophyll- $a$ and environmental variables and leveraged using the observed 
linear relationship between surface chlorophyll- $a$ and phycocyanin pigment concentrations in western Lake Erie to estimate water column cyanoHABs. A threshold value of $18 \mathrm{mg}$ $\mathrm{m}^{-3}$ of chlorophyll- $a$ was used to classify pixels as cyanoHAB based on a segmented regression analysis (Sayers et al. 2016). Briefly, using in-situ data from three eutrophic basins in the Great Lakes (Green Bay, Saginaw Bay, and western Lake Erie), Sayers et al. (2016) established a significant linear relationship between chlorophyll-a and phycocyanin when chlorophyll-a was greater than $18 \mathrm{mg} \mathrm{m}^{-3}$. The authors noted there was little to no abundance of phycocyanin for chlorophyll-a concentrations less than $18 \mathrm{mg} \mathrm{m}^{-3}$. MODISestimated water temperature was also used to distinguish between blooms of cyanobacteria $\left(>20^{\circ} \mathrm{C}\right)$ and other phytoplankton such as diatoms and green algae $\left(<20^{\circ} \mathrm{C}\right)$ (Sayers et al., 2016).

Historically, cyanoHAB mapping algorithms in the Great Lakes have not differentiated surface algal scums from sub-surface cyanobacteria in the water column (Sayers et al., 2016). In situ monitoring in Lake Erie has consistently indicated that the floating algae mats, or surface scums, that sometimes form during cyanoHAB events in Lake Erie contain extremely high concentrations of Microcystis which has the potential to affect public health. Thus, the second approach described in Sayers et al. 2016, the surface scum index (SSI, equation 1) used a band ratio index to detect cyanoHAB surface scums:

$$
\text { (1) } S S I=\left(\frac{(N I R)-(V I S)}{(N I R)+(V I S)}\right)>0
$$

where NIR represents spectral reflectance in a near-infrared satellite band and VIS represents reflectance in a visible-range band, usually red. Positive values in known water pixels were classified as surface cyanobacteria scum based on the high reflectance of algae scum at NIR wavelengths relative to low red reflectance controlled by chlorophyll (and other pigments) absorption. Other researchers have used similar approaches to successfully detect surface algae in other freshwater regions throughout the world using sensor specific methodology (Matthews, Bernard, and Robertson 2012; Hu 2009; Hu et al.2010; Peng, Wang, and Jiang 2008). It should be noted that there is no procedure for differentiating 
cyanobacteria scum from other floating macrophytes either living or not living, however, due the relatively large pixel size (250 $\mathrm{m}$ for MODIS) it would require a significant bed or wrack for floating macrophytes to trigger a positive SSI value. MODIS bands 9-13 (band centers: 443, 488, 531, 547, $667 \mathrm{~nm} ; 1 \mathrm{~km}$ resolution) were used in the $\mathrm{MCH}$, and MODIS bands 1 and 2 (645 and $858 \mathrm{~nm}$, respectively; $250 \mathrm{~m}$ resolution) were used in the SSI.

\subsubsection{Satellite Sensor Inter-calibration}

To generate cyanoHAB maps from SeaWiFS imagery, the MCH/SSI approach was initially applied with minimal modification, using bands 2-6 (band centers: 443, 490, 510, 555, and $670 \mathrm{~nm} ; 1 \mathrm{~km}$ resolution) for the $\mathrm{MCH}$, bands 6 and 8 (670 and $865 \mathrm{~nm}$, respectively; 1 $\mathrm{km}$ resolution) to calculate the SSI, and AVHRR SST as the water temperature input. This initial product was compared with the previously validated, MODIS-derived product for a selection of dates from 2002-2007 when both sensors collected cloud-free images. Comparing the outputs for the two sensors indicated that scum was initially underclassified in SeaWiFS imagery, a problem that was corrected through a series of empirically-based adjustments.

First, the MODIS-derived SSI product was compared to two SeaWiFS-derived SSI products: the original version, using SeaWiFS band 8 centered at $865 \mathrm{~nm}$ as the NIR input to the SSI algorithm, and a second version using band 7 centered at $765 \mathrm{~nm}$. It was determined that the $765 \mathrm{~nm}$ SeaWiFS SSI was more similar to the MODIS product based on Spearman rank correlation of SSI values for matched grid cells. Second, it was observed that the SeaWiFS SSI was systematically lower than MODIS SSI, in part due to the differences in sensor design characteristics (bandwidth, signal-to-noise, etc...) between MODIS Aqua and SeaWiFS. To compensate for these differences and obtain a SeaWiFS SSI product comparable to the MODIS product, the empirical relationship between the SeaWiFS and MODIS SSI products over the overlap period between the two datasets (2002-2010) was used to apply an adjustment factor to the threshold value at which SeaWiFS SSI is classified as scum. Third, these changes to the SeaWiFS SSI created problems with some scum areas being misclassified as clouds; this was corrected with a 
simple threshold filter using the cumulative reflectance of SeaWiFS visible bands. Fourth, several pixels along the shoreline were consistently flagged as scum, even outside of the cyanoHAB season, due to spatial resolution differences between SeaWiFS and the MODIS-based land mask utilized in the MCH/SSI method. This was resolved by editing the land mask to exclude those pixels, yielding a slightly smaller mapped water surface area. The cumulative effect of these adjustments is illustrated by Figure 5.2. Finally, the absorption coefficients included in the hydro-optical model utilized for the CPA-A were also adjusted by comparing the results for MODIS and SeaWiFS for the same dates and applying a vicarious calibration correction.
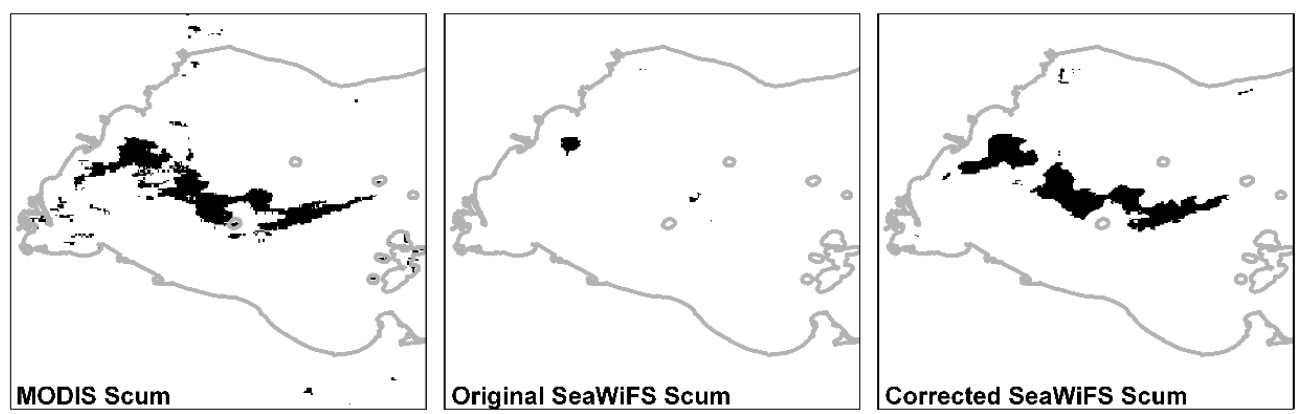

Figure 5.2. Comparison of SSI-based scum classification results from MODIS (left panel), the initial SeaWiFS result (center panel), and the corrected SeaWiFS result (right panel) for June 22, 2003. Black cells represent positive surface scum observations.

\subsubsection{SeaWiFS Product Verification}

In situ chlorophyll- $a$ measurements collected in western Lake Erie during the cyanoHAB season (August-September) between 1997 and 2007 were retrieved from EPA's Great Lakes Environmental

Database

(GLENDA, http://www.epa.gov/glnpo/monitoring/data_proj/glenda/), from the NOAA Great Lakes 
Environmental Research Laboratory's International Field Years on Lake Erie (IFYLE) Cruise Database (Hawley et al., 2006; https://www.glerl.noaa.gov/res/projects/ifyle/), and from the University of Toledo's Western Erie cruise database (Bridgeman et al., 2013). In situ surface water chlorophyll- $a$ measurements greater than $18 \mathrm{mg} / \mathrm{m}^{3}$ when the water temperature was greater than $20^{\circ} \mathrm{C}$ were considered to potentially represent a cyanoHAB based on previous analyses (Wynne et al., 2013; Sayers et al., 2016). Because presence of surface scum was not recorded, SSI was not evaluated separately from bloom presence/absence. Table 1 below summarizes the combined GLENDA, IFYLE and UT in situ data set used to evaluate the SeaWiFS MCH product. The product was evaluated by comparing each in situ observation to the value of the individual SeaWiFS pixel that the observation falls within. Observations were compared to the SeaWiFS image collected closest in time to the observation, up to a maximum of 3 days. It should be acknowledged that a single in situ observation may or may not be spatially representative of the water encapsulated within a single satellite pixel, $\sim 1 \mathrm{~km}$ in the case of SeaWiFS, however it is difficult to quantify this uncertainty with the available data. A previous study conducted a sensitivity analysis to confirm that using in situ observations taken within three days of an image collection for algorithm evaluation purposes does not decrease estimated algorithm accuracy relative to using observations from within 12 hours or one day (Ho et al., 2017a).

Table 5.1. Numbers of available in situ water quality samples measured in the western basin of Lake Erie within 3 days of a cloud-free SeaWiFS image collection during a cyanoHAB season between 1998 and 2007. There were no valid matchups in 2000 or 2001 .

\begin{tabular}{llllllllll}
\hline Year & $\mathbf{1 9 9 8}$ & $\mathbf{1 9 9 9}$ & $\mathbf{2 0 0 2}$ & $\mathbf{2 0 0 3}$ & $\mathbf{2 0 0 4}$ & $\mathbf{2 0 0 5}$ & $\mathbf{2 0 0 6}$ & $\mathbf{2 0 0 7}$ & Total \\
\hline HAB & 0 & 0 & 8 & 12 & 15 & 4 & 4 & 4 & 47 \\
No HAB & 6 & 12 & 11 & 16 & 11 & 20 & 11 & 9 & 96 \\
Total & 6 & 12 & 19 & 28 & 26 & 24 & 15 & 13 & 143 \\
\hline
\end{tabular}

\subsubsection{SeaWiFS and MODIS Product Inter-comparison}

The SeaWiFS- and MODIS Aqua-derived cyanoHAB products were compared at both the pixel and basin scales. First, the similarity of the classified map products (based on a simple 
matching coefficient) was calculated for 89 pairs of images collected on the same day between 2002 and 2007 to quantify the similarity of bloom identification at specific locations between sensors. The time difference between the two image collections ranged from 11 seconds to 1 hour 46 minutes with a mean of 30 minutes. Pixels flagged as cloud cover were excluded from the comparison. Second, bloom areas were compared across this same set of date-matched image pairs to evaluate the similarity of mapped bloom magnitudes between sensors. Coefficients of determination and root mean squared errors from weighted least squares regression between the SeaWiFS- and MODIS-estimated annual mean surface scum and total bloom areas were used to assess map similarity. Regression weights were based on the number of pixels flagged as clouds in the SeaWiFS image to account for the increase in uncertainty with cloud cover. Weights were calculated as $w_{i}=1-(c / a)$, where $w_{i}$ is the weight applied for the $i$ th date-matched image pair, $c$ is the cloud-covered area in the SeaWiFS image $\left(\mathrm{km}^{2}\right)$ and $a$ is the total mapped area in the Western Basin $\left(2,984 \mathrm{~km}^{2}\right)$. Finally, a similar regression was performed using only the annual maximum surface scum and total bloom areas for each sensor.

\subsubsection{Time-series Analysis}

After a quantitative comparison between the sensors' derived products data was combined to form a 20-year time series, extending the 12-year record reported by Sayers et al. (2016). The SeaWiFS data was used from 1998-2001 and the MODIS Aqua data was used from 2002-2017. Annual mean cyanoHAB and scum extent were determined by taking the mean extent of high chlorophyll (based on the MCH method) and scum (based on the SSI method), respectively, in all cloud-free images within the 20 degree surface temperature window. Annual maximum extents for each product were determined by taking the mean

of the largest three extents for each year. Seasonal trends were studied by breaking each year into 20 8-day windows ranging from mid-May to mid-October and finding the mean extents within each window.

In WBLE, increases in cyanoHABs had previously been linked to nutrient loading and climate change (Stumpf et al., 2012; Michalak et al., 2013). Using the MODIS Aqua 
cyanoHAB algorithm (described in the Bio-optical model section), for imagery collected from 2002 to 2013, Sayers et al. (2016) found that mean annual MCH and SSI extents were significantly related to mean spring (March-June) discharge. This analysis extended those comparisons to the longer 20-year SeaWiFS/MODIS time series. Two discharge metrics were used in determining this relationship. The first method was to use annual spring (March-June) discharge, consistent with historic discharge modeling (Stumpf et al., 2012; Sayers et al., 2016). The second method was to use half-weighted March-June discharge and add in fully weighted July discharge only when the June water temperatures were found to be above 20 degrees Celsius. This discharge formulation comes from Stumpf et al. (2016) and was found to more accurately predict bloom severity._Sayers et al. found a significant linear relationship between discharge and $\mathrm{MCH}$ and a significant cubic relationship between discharge and SSI (2016). Stumpf et al. found that discharge and CI were best modeled with an exponential model $(2012 ; 2016)$.

Mean daily discharge from the Maumee River was obtained from USGS observations (http://waterdata.usgs.gov/nwis/) using station 04193500 at Waterville, Ohio. Monthly discharge data were derived as the sum of the daily discharges. June water temperature was determined based on satellite imagery, averaging the surface water temperatures of the southern section of the western basin as defined in Stumpf et al. (2016) - south of the north end of Pelee Island (41.914 N) and west of Marblehead (82.741 W). Monthly SST data from 1998-2002 were generated from daily AVHRR data composited using the GLSEA model (Schwab et al., 1999). For 2003-2017, MODIS Aqua Level 3 Monthly $4 \mu$ SST (downloaded from the NASA OceanColor data portal) was used. Regression analyses between each discharge metric and mean annual cyanoHAB and scum extents were performed in $\mathrm{R}$ to determine which regression model is most explanatory and whether either discharge metric is an effective indicator of cyanoHAB extent. 


\subsection{Results}

The Julian days of cloud free images for SeaWiFS (1998-2001) and MODIS (2002-2017) are shown in Figure 5.3 as black dots. The date at which the water temperature exceeded and fell below $20^{\circ} \mathrm{C}$ are identified as an $\mathrm{X}$ symbol on each line, with the left most and right most X symbols representing the onset and offset of 20 degrees respectively. In some years (e.g. 2007, 2016) there are several images just outside of the satellite observed 20 degree water temperature period. These dates were included as buoy temperature measurements were still above 20 degrees. Cloud free images are generally well distributed throughout the cyanoHAB season, however, there are several periods of long-term cloud cover (e.g. September 2006, July 2013) that prohibited the estimation of cyanoHAB extents in these periods. Typically, there were fewer quality images obtained for the SeaWiFS period partially due to clouds as well as imperfect atmospheric correction. 


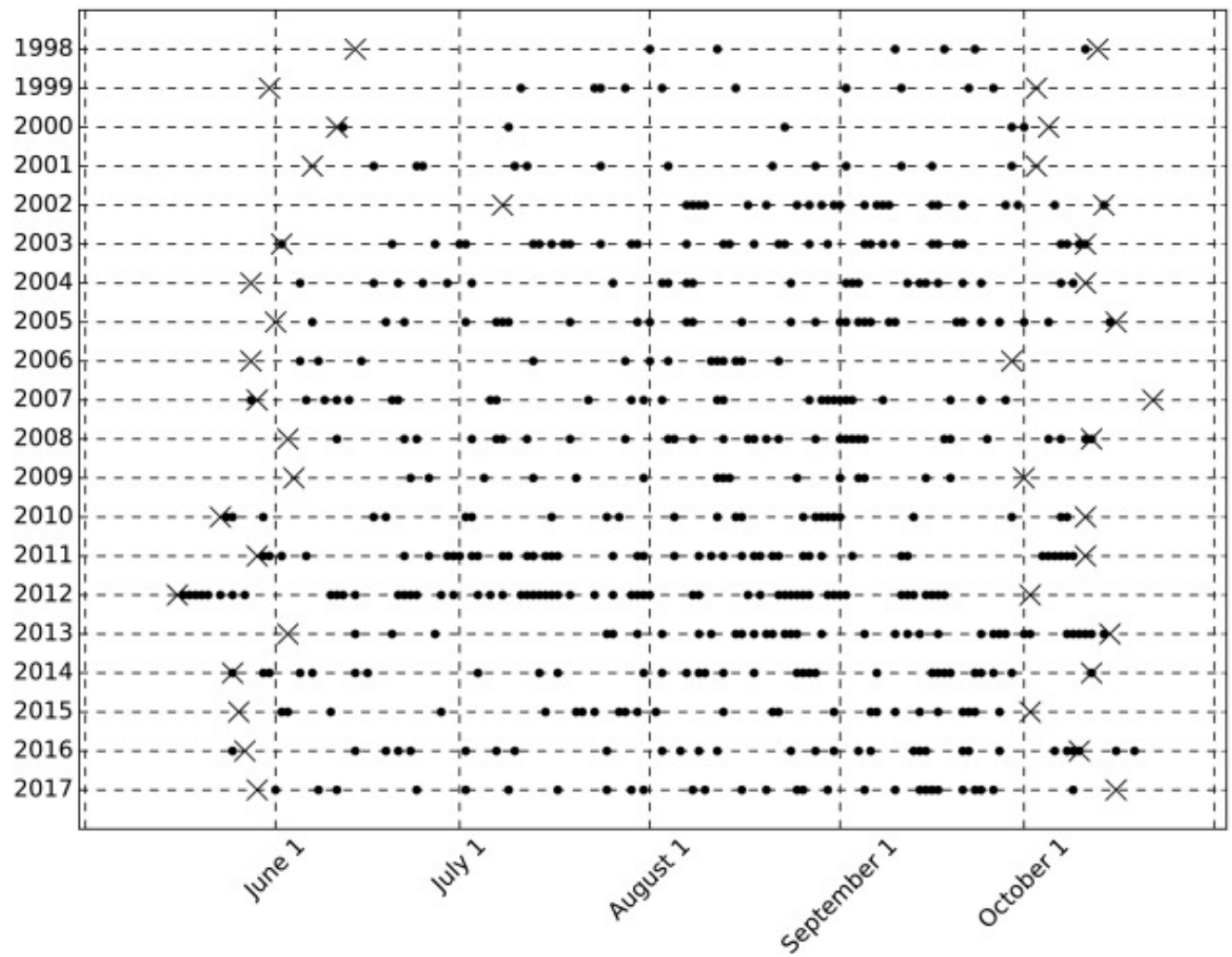

Figure 5.3. Cloud free images (black dots) used to generate combined 20 year time series data set. SeaWiFS images were used from 1998-2001 and MODIS from 2002-2017. Also shown (black X) are the 20 degree onset and offset dates established from satellite surface water temperature observations.

The SeaWiFS-derived cyanoHAB mapping products agreed well with the in situ observations (Table 5.2). CyanoHAB events were defined as remote sensing derived pixel values and in situ measurements with chlorophyll concentration greater than $18 \mathrm{mg} / \mathrm{m}^{3}$. The overall accuracy of $81 \%$ is slightly lower than that obtained for the MODIS Aqua product $(87 \%$ for $\mathrm{MCH}$; Sayers et al., 2016). False positive and false negative classifications were equally frequent. Classification accuracy was lower when a cyanoHAB was present $(70 \%)$ than when absent (86\%). Misclassified pixels tended to be located near the edge of a bloom. There was no strong seasonal trend in validation accuracy. This assessment confirms the suitability of SeaWiFS for the $\mathrm{MCH}$ approach to cyanoHAB mapping. 
Table 5.2. Classification error matrix for SeaWiFS-derived MCH vs. in situ observations. The hybrid algorithm agreed with 116 out of 143 in situ observations for an overall accuracy of $81 \%$ and Kappa coefficient of 0.57 .

\begin{tabular}{|c|c|c|c|c|c|c|}
\hline & \multicolumn{5}{|c|}{ Field Truth } \\
\hline & & HAB & No HAB & Total & $\begin{array}{c}\text { User's } \\
\text { Accuracy }\end{array}$ & $\begin{array}{c}\text { Commission } \\
\text { Error }\end{array}$ \\
\hline \multirow{6}{*}{ } & HAB & 33 & 13 & 46 & $72 \%$ & $28 \%$ \\
\hline & No HAB & 14 & 83 & 97 & $86 \%$ & $14 \%$ \\
\hline & Total & 47 & 96 & & & \\
\hline & Producer's & $70 \%$ & $86 \%$ & & Overall & $81 \%$ \\
\hline & Accuracy & & & & Accuracy & \\
\hline & $\begin{array}{c}\text { Omission } \\
\text { Error }\end{array}$ & $30 \%$ & $14 \%$ & & Kappa & 0.57 \\
\hline
\end{tabular}

At the pixel scale, the average similarity of the 89 date-matched pairs of images was $89 \%$ (simple matching coefficient, SMC). There was no significant relationship between SMC and the time difference between MODIS Aqua and SeaWiFS collections (Pearson's $r=-$ $0.12, \mathrm{n}=89, \mathrm{p}=0.28$ ). This is in line with expectations given the similar overpass times of SeaWiFS and MODIS Aqua for Lake Erie, and supports the integration of SeaWiFSand MODIS Aqua-derived cyanoHAB estimates into a single time series.

At the basin scale, the weighted coefficient of determination $\left(\mathrm{w} R^{2}\right)$ and the root mean squared error (RMSE) for the weighted linear regressions of the mean basin wide bloom area date-matched pairs $(\mathrm{n}=89)$ indicated relatively strong agreement at 0.81 and $297 \mathrm{~km}^{2}$ respectively. These uncertainty values are similar to those reported by Ho et al. 2017a when comparing Landsat and MERIS cyanoHAB extents. The coefficient of determination $\left(\mathrm{R}^{2}\right)$ and RMSE for annual maximum areas $(\mathrm{n}=6)$ were 0.74 and $421 \mathrm{~km}^{2}$, respectively. The annual maximum extent RMSE $\left(421 \mathrm{~km}^{2}\right)$ between the SeaWiFS and MODIS observations was approximately $14 \%$ of the mappable area of the basin $\left(2984 \mathrm{~km}^{2}\right)$. This result is reasonable considering that the maximum extent observed by each sensor may not have occurred on the same day, for example in 2002 the SeaWiFS maximum extent was observed on August $8^{\text {th }}$ while the MODIS maximum was on August $31^{\text {st }}$. The discrepancy between sensor observed maxima are partially due to cloud formation between sensor 
overpasses or suboptimal viewing geometry of one platform relative to the other as well as shoreline effects. While there is no established threshold criteria to evaluate successful multi-sensor ocean color product merger (IOCCG, 2007), the agreement $\left(\mathrm{w} R^{2}\right)$ between SeaWiFS and MODIS cyanoHAB merged products identified in this study is similar to those identified for other global ocean color product mergers.

Finally, the SeaWiFS and MODIS Aqua products for the overlapping 2002-2007 years, described in the SeaWiFS and MODIS Product Inter-comparison section, were compared based on the annual mean and maximum cyanoHAB areas (Figure 5.4a, top panel) and surface scum areas (Figure 5.4b, bottom panel). Trends in mean and maximum annual cyanoHAB area, as derived from the $\mathrm{MCH}$ algorithm, were very similar between sensors, with a mean difference of $16 \%$ in overlapping years for both metrics. Surface scum area showed more divergence between sensors, with $68 \%$ and $64 \%$ difference in mean and maximum area, respectively, in overlapping years. The greater disparity in scum areas between sensors is potentially due to the difference in sensor spatial resolution (i.e. MODIS is $250 \mathrm{~m}$ and SeaWiFS is $1000 \mathrm{~m}$ ) and the difference in image acquisition time (up to 1 hour and 45 minutes). CyanoHAB surface scums are highly dynamic in their vertical and horizontal distribution forming patches which can quickly advect or mix down into the water column making it problematic to observe similar spatial distributions over short timescales (Bosse et al., this issue; Lekki et al., this issue). The trends do track fairly well between sensors for these metrics though, with the only clear difference being the maximum scum extent in 2003 where SeaWiFS maximum observed extent was almost two-fold greater than that observed by MODIS. 

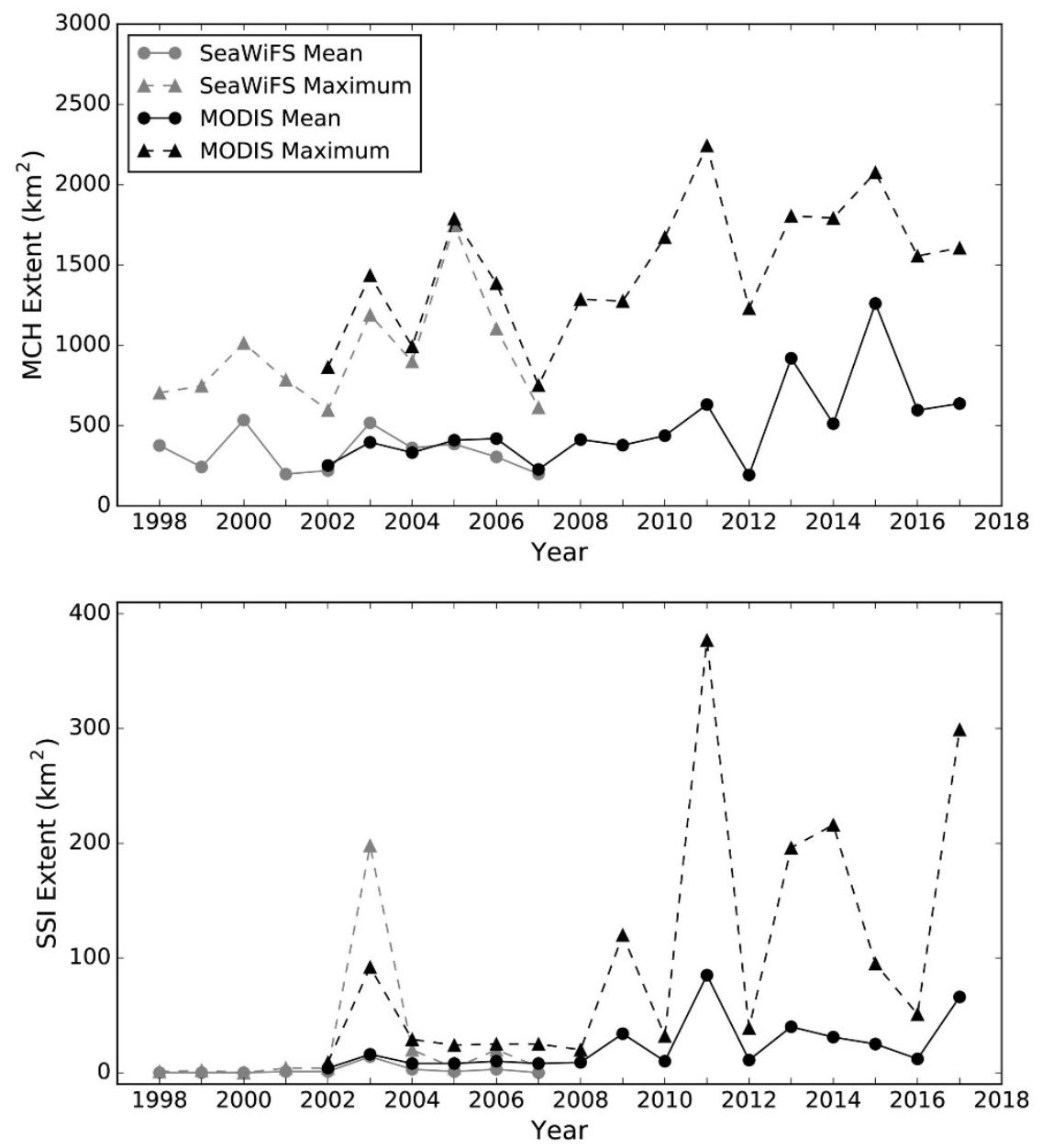

Figure 5.4. Comparison of mean and maximum annual cyanoHAB areas based on the MCH (a, top panel) and surface scum areas based on the SSI (b, bottom panel) in the Western Basin of Lake Erie for SeaWiFS (1998-2007) and MODIS Aqua (2002-2017).

Combining the SeaWiFS (1998-2001) and MODIS Aqua (2002-2017) data into annual 20 year time-series datasets, the mean annual $\mathrm{MCH}$ cyanoHAB extent and maximum $\mathrm{MCH}$ annual cyanoHAB extent trends along with linear fits and 95\% confidence intervals were calculated and are shown in Figure 5.5. A significant linear relationship was identified between year and mean annual extent (Figure 5.5a, left panel, slope $=25.28, \mathrm{R}^{2}=0.34$, $p=0.007$ ), however, it is only explaining $34 \%$ of the observed variability. The mean annual extent was consistent between 200 and $500 \mathrm{~km}^{2}$ (representing between 7 and 17\% of the 
WBLE) from 1998 to 2010, however, beginning in 2007, the mean extent displayed an upward trend, peaking in 2015 when the mean cyanoHAB extent exceeded $42 \%$ of the basin. Recent years (2010-2017) have shown increased year-to-year variability (Figure $5.5 \mathrm{a}$, left panel), as indicated by the dramatic up and down cycling of the mean annual extents. The mean percent difference between consecutive annual extent pairs for the 19982009 period was $43.9 \%$ while the $2010-2017$ period was $75.8 \%$, an approximate $76 \%$ increase in year-to-year variability between the early (1998-2009) and more recent (20102017) periods. The observed increase in inter-annual variability is also evident from the large deviation in mean annual extents from the 95\% confidence interval in 2012, 2013, and 2015. Regression model statistics are summarized in Table 5.3.

The maximum cyanoHAB extent (Figure 5.5b, right panel) has been consistently and significantly increasing over the 1998-2017 period (slope $=57.7, \mathrm{R}^{2}=0.54, \mathrm{p}<0.001$ ). The maximum observed cyanoHAB extent has increased more than two-fold (linear trend predicted value for $1998=802 \mathrm{~km}^{2}$ and $2017=1899 \mathrm{~km}^{2}$ ) from 1998-2017. Approximately $54 \%$ of the observed variability in maximum annual extent is explained by year, which is almost $60 \%$ more than for the mean annual extent relationship (Figure 5.4a, left panel). The annual maximum extent also showed significant variability, with five years (2003, 2005, 2007, 2011, and 2012) falling outside (both above and below) of the $95 \%$ confidence interval. Regression model statistics are summarized in Table 5.3.

Table 5.3. Linear regression statistics for the 20 -year time series mean and maximum $\mathrm{MCH}$ and SSI annual extents.

\begin{tabular}{lccc}
\hline \multicolumn{1}{c}{ Extent } & Slope & $\mathbf{R}^{\mathbf{2}}$ & P-value \\
\hline Mean MCH & 25.28 & 0.34 & 0.007 \\
Maximum & 57.7 & 0.54 & $<0.001$ \\
MCH & 2.42 & 0.39 & 0.003 \\
Mean SSI & 11.29 & 0.39 & 0.003 \\
Maximum SSI & & & \\
\hline
\end{tabular}



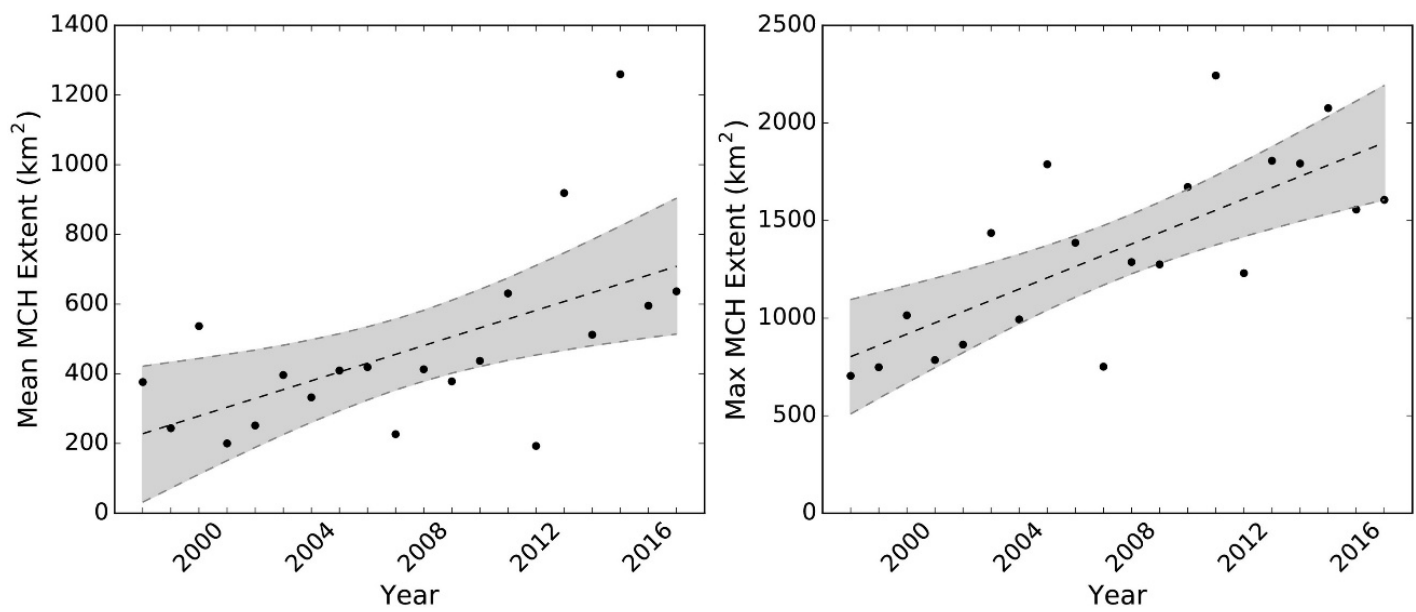

Figure 5.5. (a, left panel) Annual mean cyanoHAB extent $\left(\mathrm{km}^{2}\right)$ from the $\mathrm{MCH}$ with a linear best fit line (slope $=25.28, \mathrm{R}^{2}=0.34, \mathrm{p}=0.007$ ) and $95 \%$ confidence interval shaded in grey and (b, right panel) annual maximum cyanoHAB extent with a linear best fit line ( lope $\left.=57.7, \mathrm{R}^{2}=0.54, \mathrm{p}<0.001\right)$ and 95\% confidence interval shaded in grey. Points from 1999-2001 come from the SeaWiFS sensor; points from 2002-2017 come from the MODIS Aqua sensor.

Trends in mean and maximum SSI extents were also derived from the combined SeaWiFS and MODIS dataset and are shown in Figure 5.6. A significant linear relationship between mean annual SSI extent and year was identified from the time-series data (Figure 5.6a, left panel, slope $=2.42, \mathrm{R}^{2}=0.39, \mathrm{p}=0.003$ ). While the trend is statistically significant, large year-to-year variability is observed in the 2010-2017 period relative to the earlier years. The maximum scum extent is also increasing significantly (Figure 5.6b, right panel, slope $\left.=11.29, \mathrm{R}^{2}=0.39, \mathrm{p}=0.003\right)$ throughout the 20 year period, however with large interannual variability similar to the mean annual scum extent. It is clear from figure 5.6 (a and b) that large surface scums of cyanoHABs are becoming larger on average in the western basin of Lake Erie. 

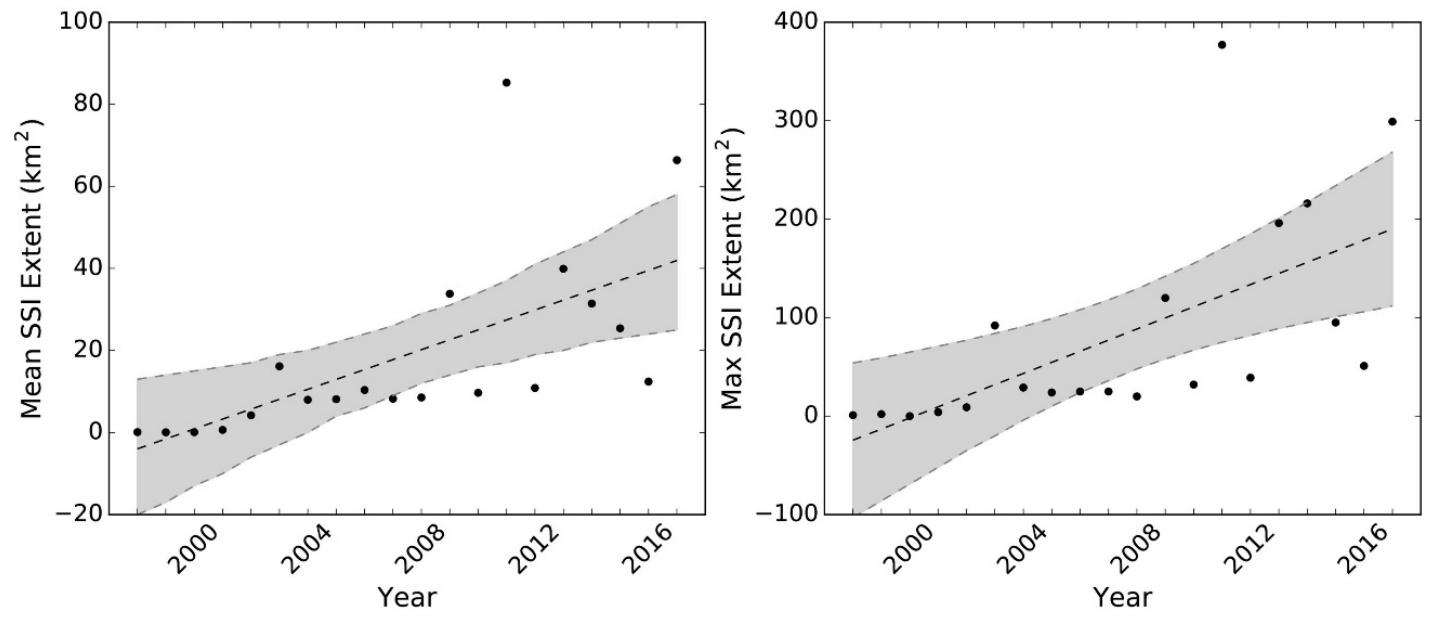

Figure 5.6. (a, left panel) Annual mean scum extent $\left(\mathrm{km}^{2}\right)$ from the SSI with a linear best fit line (slope $=2.42, \mathrm{R}^{2}=0.39, \mathrm{p}=0.003$ ) and $95 \%$ confidence interval shaded in grey and (b, right panel) annual maximum cyanoHAB extent with a linear best fit line ( lope $=11.29, \mathrm{R}^{2}=0.39, \mathrm{p}=0.003$ ) and $95 \%$ confidence interval shaded in grey. Points from 1999-2001 come from the SeaWiFS sensor; points from 2002-2017 come from the MODIS Aqua sensor. Note the linear fits in the figure are not intended for scum area prediction.

In addition to significant interannual variability, satellite derived cyanoHAB areal extents are highly variable within years (Figure 5.7a, left panel). Intra-annual cyanoHAB variability (standard deviation of observed bloom areas within each year) varied from 221 $\mathrm{km}^{2}$ to $633 \mathrm{~km}^{2}$ with a mean of $442 \mathrm{~km}^{2}$. Large intra-annual variability was observed for both the early 1998- 2010 period and the more recent 2011-2017 period indicating no discernible changes or trends in seasonal variability across the combined 20 year timeseries. Some of the observed differences in intra-annual variability between years is the product of a differing number of clear satellite observations (e.g. 13 images in 2001 and 25 in 2015) as well as the timing of available clear observations (e.g. 2001 had 3 August images and 2015 had 5 August images) while the remaining variability is likely due to seasonal variations in bloom extent. 
The seasonal variability of cyanoHAB extent was examined using the combined 20 year time series binned into 8-day composite values. Figure 5.7b (right panel) shows the seasonal progression of cyanoHAB extent, derived from the full 20 -year data record. This progression shows generally small cyanoHAB extents $\left(<300 \mathrm{~km}^{2}\right)$ May through June (Day 120-180), followed by a steady increase from mid-July (Day 195) until the cyanoHAB area peaks in mid- to late-September (Day 255-270) and begins to decline until the end of the season. Variability in extent is evident in each of the 8-day periods with greater variance $\left(453 \mathrm{~km}^{2}\right)$ occurring once the bloom has initiated (i.e. after day 200) relative to preinitiation variance $\left(185.5 \mathrm{~km}^{2}\right)$.
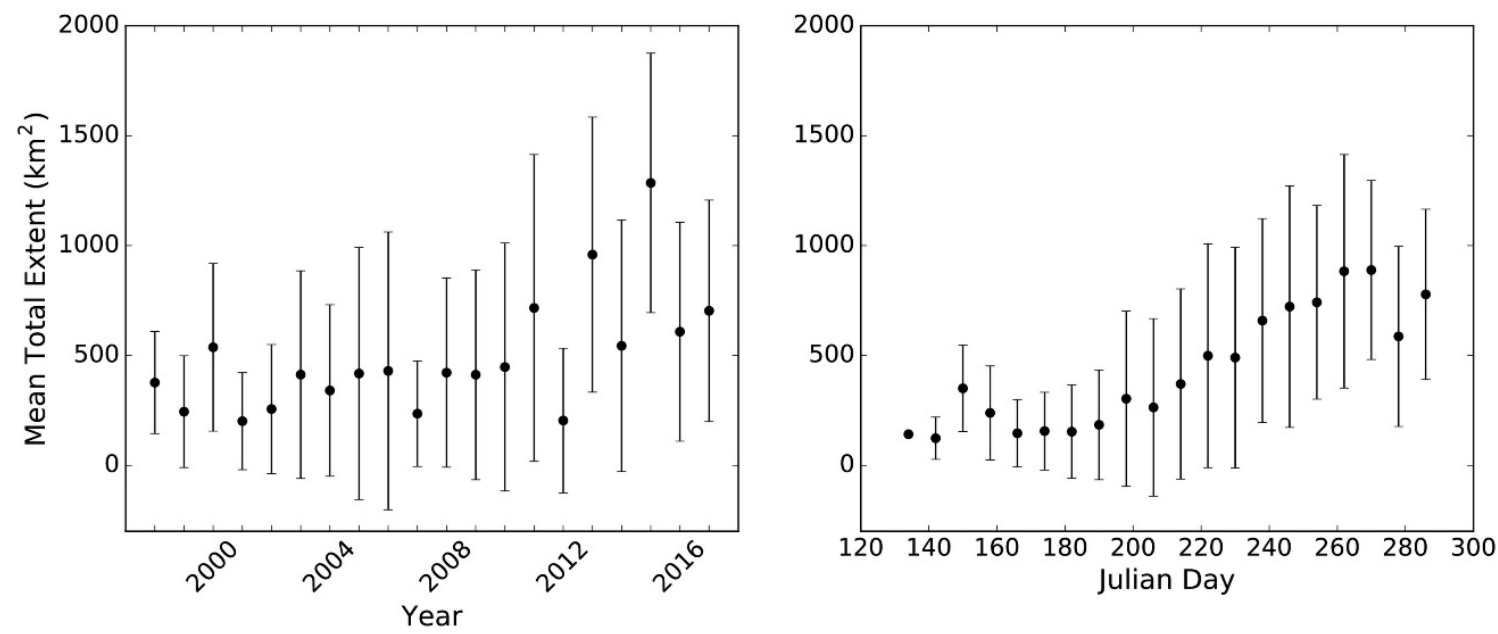

Figure 5.7. a, left panel, Mean total cyanoHAB extent (floating and nonfloating) over the 20 year data record in Western Basin Lake Erie plotted with standard deviation errors. $b$, right panel, Mean 8-day seasonal pattern of total cyanoHAB extent in Western Basin Lake Erie, plotted with standard deviation error bars.

The spatial and temporal extent of cyanoHABs during the 20 year period in the western basin can be easily examined with the use of heat maps. A heat map is simply the frequency of occurrence of cyanoHABs within each pixel for a given year. Figure 5.8 presents annual heat maps for the 1998-2017 period. These annual maps show the increasing extent of cyanoHABs across the basin as well as their spatial patterns which appear to be focused 
around the mouth of the Maumee River and extend east along the southern edge of the lake while rarely traveling north into the outflow of the Detroit River. The SeaWiFS period (1998-2001) heat maps all show large areas where no cyanoHAB was observed (shown black) within a given year. With the exception of 2007, the large area of cyanoHAB-free water in the western basin has dramatically decreased since 1998, with 2017 exhibiting only $5 \%$ percent of the basin as free of cyanoHAB. 


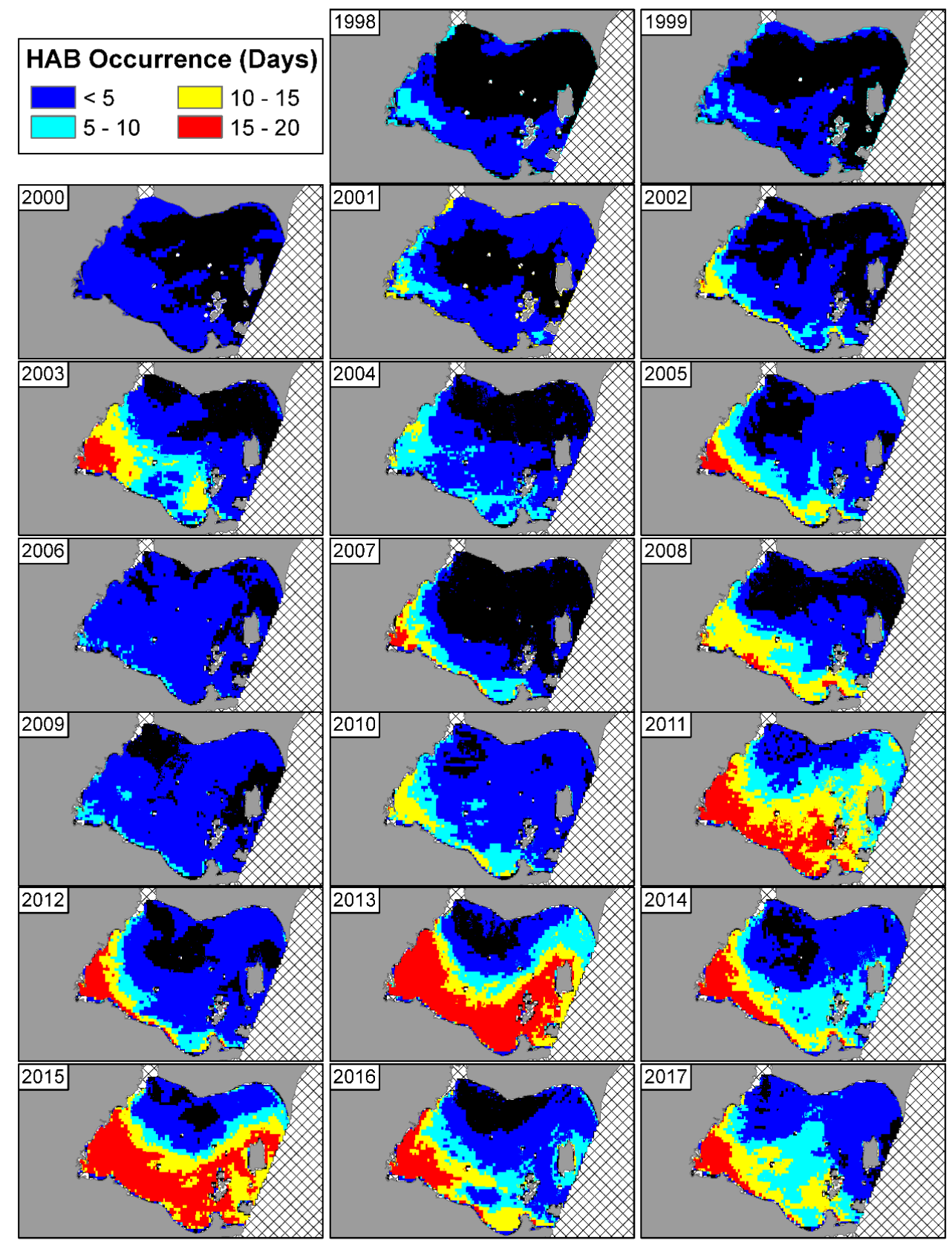

Figure 5.8. Frequency occurrence annual heat maps of cyanoHAB presence for the 1998-2017 period. Areas of more frequent occurrence are shown in warmer colors while areas of no-occurrence are shown in black. Data from 1999-2001 come from the SeaWiFS sensor; data from 2002-2017 come from the MODIS Aqua sensor. 
Simple heat maps can illustrate the cumulative occurrence of cyanoHABs across the basin over the combined 1998-2017 time series (Figure 5.9). The total cyanoHAB heat map (figure 5.9 left panel) is comprised of both water column mixed (MCH) and surface scum (SSI) mapped pixels and represented in weeks of occurrence. The most frequent cyanoHAB occurrences are concentrated in the Michigan and Ohio nearshore areas extending approximately $12-16 \mathrm{~km}$ into the basin. Significant occurrences ( $8-16$ weeks) are also observed extending outward to the middle of the basin and amongst the island archipelago to the east. Very few ( $<4$ weeks) cyanoHAB occurrences were detected in the proximity of the Detroit River plume (light and dark blue). The surface scum heat map demonstrates the very limited temporal and spatial extent of these events in the western basin during the 1998-2017 period (Figure 5.9 right panel). Surface scums were prevalent (more than 8 days) in the mouth of the Maumee River extending just up the Michigan and Ohio shorelines. There was also significant occurrence of scums near the islands in the middle and eastern end of the basin possibly due to increased accumulation from prevailing winds and underlying currents. Interestingly, there are very few scum occurrences along the southern shore of the basin just north of Sandusky Bay which is in contrast with the total cyanoHAB cumulative distribution that shows wide-spread presence. Finally, very few scum forming blooms are observed in the northern half of the basin in the vicinity of the Detroit River outflow. 

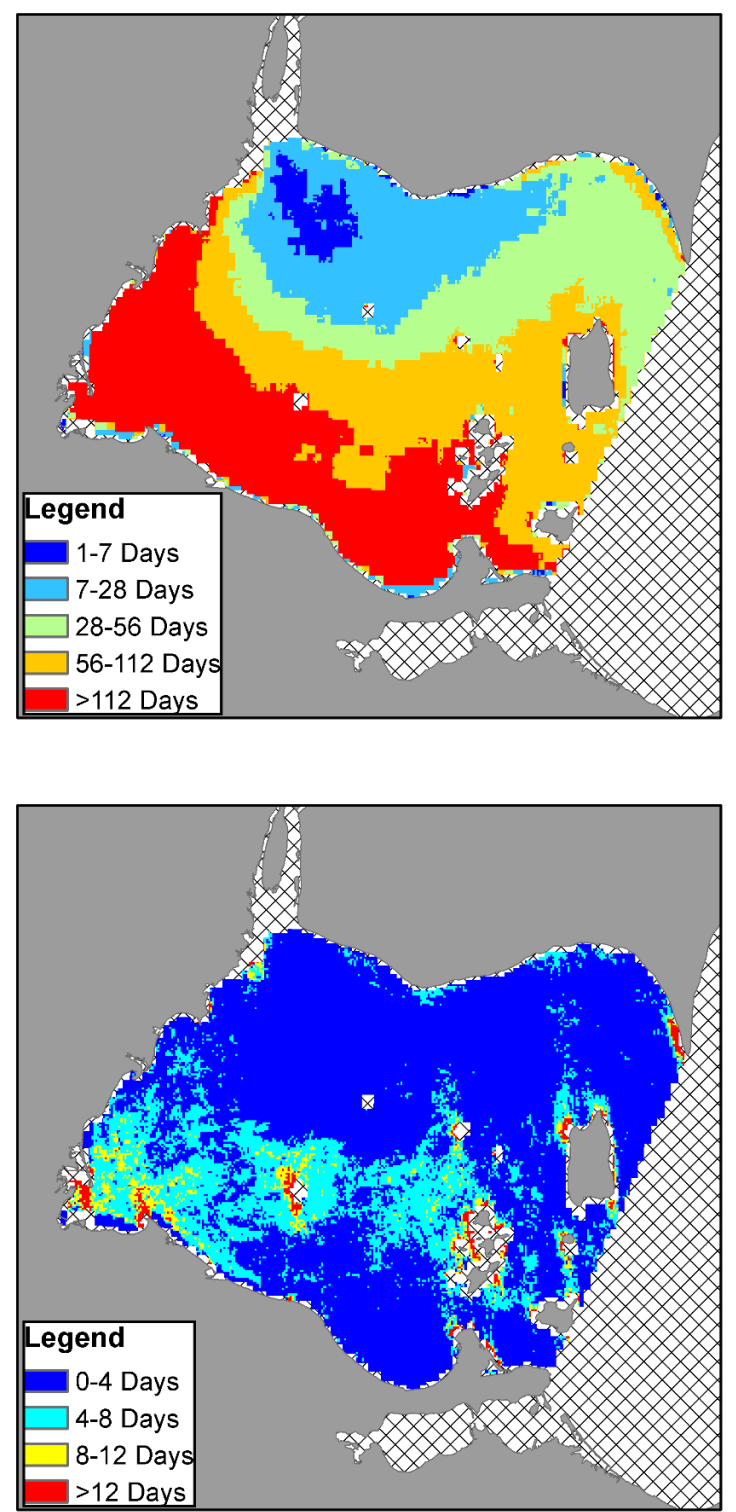

Figure 5.9. Cumulative frequency occurrence heat maps of total cyanoHABs (left) and surface scum (right) for the 1998-2017 period. Warmer colors indicate more frequent occurrence.

In order to better understand drivers controlling mean annual cyanoHAB extent, several previously defined metrics of mean spring discharge for the Maumee River as well as fit types were compared to annual MCH and SSI values for the Western Basin of Lake Erie for 1998-2017 period. In all cases the mean weighted spring discharge metric, $w$ Q, (Stumpf 
et al. 2016) produced more significant relationships with cyanoHAB extent than the mean March-June discharge metric, $\mathrm{Q}(\mathrm{MCH}$ linear $-w \mathrm{Q} \mathrm{p}=0.01, \mathrm{Q}=0.019$; $\mathrm{MCH}$ exponential - $w \mathrm{Q} \mathrm{p}=0.002, \mathrm{Q} \mathrm{p}=0.009 ;$ SSI cubic $-w \mathrm{Q} \mathrm{p}<0.001, \mathrm{Q} \mathrm{p}=0.004$, SSI exponential $-w \mathrm{Q}$ $\mathrm{p}=0.002, \mathrm{Q} \mathrm{p}=0.011$ ). The relationships between cyanoHAB extents and $w \mathrm{Q}$ are shown in figure 5.10. A significant linear relationship was found between $w \mathrm{Q}$ and $\mathrm{MCH}(\mathrm{y}=1.53 \mathrm{x}$ $+64.14, \mathrm{p}=0.01, \mathrm{R}^{2}=0.27$; Figure $5.10 \mathrm{a}$ ). While significant, $w \mathrm{Q}$ only explains $27 \%$ of the variance in mean annual $\mathrm{MCH}$ extent due in part to the two extremely large extent years in 2013 and 2015. A significant exponential fit between $w \mathrm{Q}$ and $\mathrm{MCH}$ annual extent was also identified $\left(\mathrm{y}=165.92 * 10^{\wedge}(0.022 \mathrm{x}), \mathrm{p}=0.002, \mathrm{R}^{2}=0.39\right.$; Figure 5.10b). The exponential model is better than the linear model for predicting $\mathrm{MCH}$ annual extent, however, it was still only able to capture $39 \%$ of the variance again in part due to the large 2013 and 2015 events. Model equations and statistics are summarized in Table 4.

The $w \mathrm{Q}$ discharge metric was also able to predict mean annual SSI extent. A significant cubic relationship was observed between $w \mathrm{Q}$ and annual SSI $\left(\mathrm{y}=0.002 \mathrm{x}^{3}+0.09 \mathrm{x}^{2}-3.11 \mathrm{x}\right.$ $+25.92, \mathrm{p}<0.001, \mathrm{R}^{2}=0.71$; Figure 5.10c). An exponential relationship was also identified between $w \mathrm{Q}$ and annual SSI $\left(\mathrm{y}=1.84^{*} 10^{\wedge}(0.047 \mathrm{x}), \mathrm{p}=0.002, \mathrm{R}^{2}=0.45\right.$; Figure 5.10d). The cubic model explains more of the variance in annual SSI extent than the exponential model (cubic $\mathrm{R}^{2}=0.71$, exponential $\mathrm{R}^{2}=0.45$ ). The cubic model is able to predict the two largest SSI extent events in 2011 and 2017 better than the exponential model as it underestimates both years. The slightly negative trend in the cubic model from $5-10 w \mathrm{Q}$ is likely unrealistic and simply an artifact of small extent variability in the lowest three observations. Model equations and statistics are summarized in Table 5.4.

Table 5.4. $\mathrm{MCH}$ and SSI extent model equations and statistics. Extent models are derived using the weighted spring discharge metric $(w \mathrm{Q})$ as the predictor.

\begin{tabular}{lccc}
\hline \multicolumn{1}{c}{ Model } & Equation & $\mathbf{R}^{2}$ & P-value \\
\hline MCH Linear & $1.53 \mathrm{x}+64.14$ & 0.27 & 0.01 \\
MCH Exponential & $165.92 * 10^{\wedge}(0.022 \mathrm{x})$ & 0.39 & 0.002 \\
SSI Cubic & $0.002 \mathrm{x}^{3}+0.09 \mathrm{x}^{2}-3.11 \mathrm{x}+25.92$ & 0.71 & $<0.001$ \\
SSI Exponential & $1.84 * 10^{\wedge}(0.047 \mathrm{x})$ & 0.45 & 0.002 \\
\hline
\end{tabular}



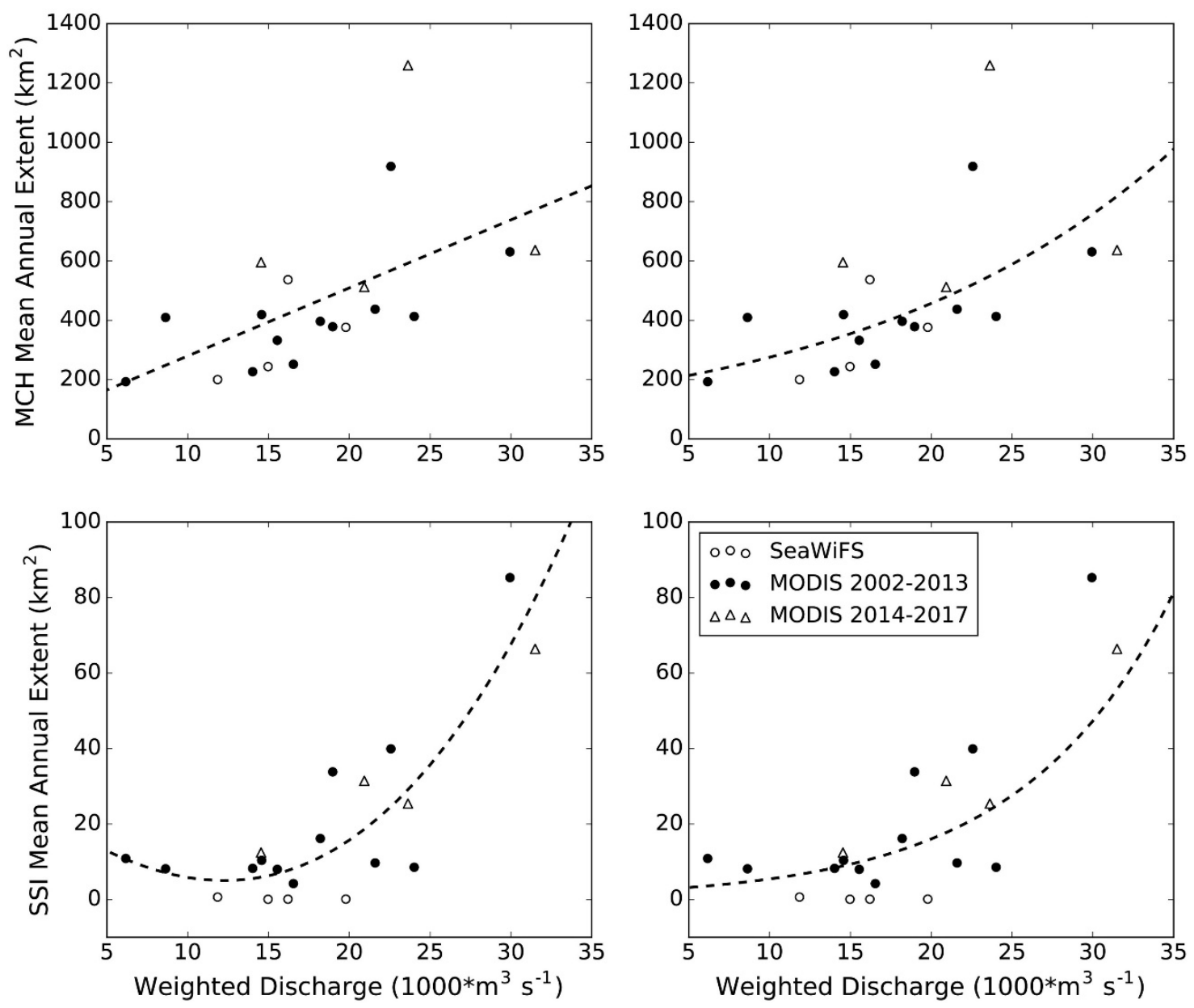

Figure 5.10. Relationships between Maumee River weighted spring discharge and (a) $\mathrm{MCH}$ mean annual extent linear fit $\left(\mathrm{y}=1.53 \mathrm{x}+64.14, \mathrm{p}=0.02, \mathrm{R}^{2}=\right.$ 0.27), (b) $\mathrm{MCH}$ mean annual extent exponential fit $\left(\mathrm{y}=165.92 * 10^{\wedge}(0.022 \mathrm{x})\right.$, $\left.\mathrm{p}=0.002, \mathrm{R}^{2}=0.39\right)$, (c) SSI mean annual extent cubic fit $\left(\mathrm{y}=0.002 \mathrm{x}^{3}+\right.$ $0.09 \mathrm{x}^{2}-3.11 \mathrm{x}+25.92, \mathrm{p}<0.001, \mathrm{R}^{2}=0.71$ ), and (d) SSI mean annual extent exponential fit $\left(\mathrm{y}=1.84^{*} 10^{\wedge}(0.047 \mathrm{x}), \mathrm{p}=0.002, \mathrm{R}^{2}=0.45\right)$. Plotted lines represent the best fits lines for each relationship. Open circles are the extents derived from SeaWiFS data, closed circles are from MODIS data reported in Sayers et al. (2016), and open triangles are from MODIS data reported in this study. 


\subsection{Discussion}

This study documents the development and validation of the first reported cyanoHAB monitoring product generated from the SeaWiFS sensor and the intercomparison with the MODIS cyanoHAB distribution product for Lake Erie. Modification of cyanoHAB/phytoplankton mapping algorithms for different remote sensing platforms and inter-comparisons of their results is not new for Lake Erie. Wynne et al. (2013) modified the Cyanobacteria Index (CI) for application with MODIS and showed generally good agreement between basin-wide cyanoHAB distributions. This modification allowed for a longer time-series (2002-2015) to be used to generate the NOAA cyanoHAB forecast model currently in operation (Stumpf et al., 2016). Similarly, Ho et al. (2017a) evaluated multiple Landsat 5 algorithms to estimate phytoplankton blooms and compared the results with those obtained from the MERIS and MODIS CI products. The Landsat 5 intercomparison allowed for a longer phytoplankton bloom time record (1984-2015) to be developed which was further used to explore the interaction of long-term internal loading of dissolved reactive phosphorus (DRP) and annual spring loadings to better predict phytoplankton blooms in western Lake Erie (Ho et al., 2017b). While the Landsat data fusion allows for a much longer time-series, the 16-day revisit time in conjunction with frequent cloud cover limit its ability to provide seasonal variability as well as robust uncertainties in annual extents. Prior to this study, SeaWiFS has not been used to map or monitor cyanoHAB presence in the Great Lakes. Other researchers have previously used SeaWiFS data to extend cyanoHAB observations in other water bodies throughout the world (Miller et al. 2006, Kahru et al. 2007; 2014) to improve understanding of long-term environmental changes. Results from these studies indicated the added value of including SeaWiFS data in long-term monitoring of cyanoHAB distributions, strongly suggesting similar value would be expected from its application in Great Lakes water bodies. Incorporation of daily revisit SeaWiFS data into the time-series extends the previously derived MODIS record (2002-2013; Sayers et al., 2016) back to 1998 providing more robust annual and seasonal observations to better understand how cyanoHAB dynamics have changed over the past 20 years. This new dataset clearly augments those cyanoHAB 
products previously developed for Lake Erie (Stumpf et al,. 2012, 2016; Sayers et al., 2016; Ho et al., 2017a).

The annual trends generated by the new SeaWiFS/MODIS fused cyanoHAB extent products are similar to those produced by prior investigators that used the MERIS sensor which has been well documented to produce robust cyanoHAB estimates (Stumpf et al., 2012; Wynne et al., 2013). There are several differences in the methodologies that can lead to different results, however. The Cyanobacteria Index (CI) uses MERIS spectral bands located at 665,681 , and $709 \mathrm{~nm}$ to relate the spectral shape in this wavelength region to abundance of cyanobacteria (Gower et al. 1999, Wynne et al., 2008). In the case of high phytoplankton/cyanobacteria biomass, particulate scattering (possibly enhanced due to cyanobacteria cell structure) begins to overwhelm the signal from pure water absorption in the red edge spectral region forming a peak $\sim 709 \mathrm{~nm}$ while phycobilipigment fluorescence overwhelms chlorophyll- $a$ absorption to form a peak around $665 \mathrm{~nm}$ (Matthews et al., 2012). These two peaks formed on both sides of the Q-band chlorophyll- $a$ absorption feature $(\sim 675 \mathrm{~nm})$ form a trough in reflectance at approximately $681 \mathrm{~nm}$ forms. The depth of this trough relative to the surrounding peaks is the basis for the CI estimation of cyanobacteria abundance. Because of the cyanobacteria particle scattering and phycobilipigment fluorescence dominance of this spectral response and the strong absorption by pure water, the $\mathrm{CI}$ requires a moderate presence of biomass before positive identification is achieved. For example, Rowe et al. (2016) related the CI low level of detection to approximately $23 \mathrm{mg} / \mathrm{m}^{3}$ of chlorophyll $-a$. The $\mathrm{MCH}$ method used in this study is a full spectrum $(412-667 \mathrm{~nm})$ inversion model that takes into account both scattering and absorption processes to retrieve chlorophyll- $a$ concentrations which are then empirically related to the cyanobacteria phycobilipigment phycocyanin (Sayers et al. 2016). The MCH was shown to be able to empirically estimate phycocyanin when chlorophyll-a concentrations were greater than $18 \mathrm{mg} / \mathrm{m}^{3}$. Therefore, the difference between $\mathrm{CI}$ and $\mathrm{MCH}$ extent values may be related to the difference in each algorithm's low level of detection of chlorophyll- $a$ biomass that in some cases can result in differences in the retrieved 
biomass/area of cyanoHAB blooms at very low concentrations that can be observed during the bloom initiation period.

Another unique aspect of this study is the generation of the cyanoHAB surface scum extent 20-year "heat map" which depicts areas experiencing intense blooms that have been associated with very high toxin levels making them a particularly high concern for public health (Bartram and Rees 1999). Scums can form and dissipate rapidly with the shifts in prevailing wind and waves (Paerl and Ustach, 1982; Wynne et al., 2013; Rowe et al., 2016; Bosse et al., 2018 this issue) making them difficult to track and characterize with in situ measurements. The SSI approach used in this study makes use of the significant nearinfrared scattering of surface algal mats to identify their presence from satellite sensors. Using the SSI to characterize scum occurrence for the 20-year time series identifies areas where scums are most often located. As expected scums are common in the mouth of the Maumee River where very shallow waters, shelter from prevailing winds, and injection of critical nutrients are common. Significant scum occurrence was also identified around the islands with the most significant accumulations on the west ends that corresponds to the direction of the prevailing summer winds (from the southwest). These observations suggest scums can be formed as wind driven currents advect near-surface particles, which rapidly accumulate as they are pushed into the island shorelines. Underlying currents and surface winds may also be responsible for the observation that scum "hot spots" do not match those identified for the total cyanoHAB extent (cells mixed in the optical depth sampled from satellite remote sensing). For example, Bosse et al. (2018, this issue) suggested surface accumulations of high biomass cyanobacteria were more frequent in deeper waters under low wind conditions whereas shallower areas $(<3 \mathrm{~m})$ were more often well mixed under similar conditions. In this scenario, persistent high biomass blooms in shallow waters may often be well mixed which can be identified with the MCH but not SSI. This may be why the large shallow (2-6 m) area along the middle of the southern shoreline shows frequent blooms with MCH (Figure 5.8) but essentially no scum occurrences (Figure 5.9) over the 20 -year period. Identification of scum "hot spots" is a new and unique aspect of this study 
that was previously unreported and will be particularly useful for regional water managers as well as for ecosystem modeling.

Annual cyanoHAB extents derived from the new 20-year fused dataset reinforces the prevailing assumption that cyanoHABs are getting worse in Lake Erie. Multiple investigators have used remote sensing and in situ sampling data to determine that cyanoHAB extents are becoming larger and more prevalent in Lake Erie (Stumpf et al., 2012; Bridgeman et al., 2013; Michalak et al., 2013; Wynne et al., 2015; Sayers et al., 2016; Stumpf et al., 2016). For example, Stumpf et al. (2012), using MERIS data, showed maximum bloom areas were larger in 2008, 2009, and 2011 than previous years back to 2002. These observations were updated by Stumpf et al. (2016) using the merged MERIS and MODIS CI products which identified very large blooms in 2013 and 2015. Similarly, Sayers et al. (2016) also identified greater mean annual cyanoHAB extent in 2011 and 2013 for the western basin of Lake Erie. Finally, Bridgeman et al. (2013) noted from in situ observations that the 2011 bloom was 29 times larger than the smallest bloom recorded since 2002.

The addition of the 2016 and 2017 annual cyanoHAB extents presented in this study showed the continuation of the cyclical pattern in extents that began between 2010 and 2011. This pattern has been evident and increasing where, until 2017, every other year saw a new "all-time record" cyanoHAB extent. The down years between the peaks were also increasing at a relatively continuous rate as well, indicating even the so called "down" (relative to the previous year) cyanoHAB years $(2012,2014,2016)$ are increasing in mean annual extent. Moreover, this study shows the substantial linear increase in maximum cyanoHAB extent throughout the time-series suggesting that even if the mean annual extent is not large there is a high probability of a very large bloom occurring for at least some period of time within each year moving forward. These results would agree with the conclusions of other researchers (Obenour et al., 2014; Matisoff et al., 2016; Watson et al., 2016; Ho et al., 2017b) that suggest other controls beyond spring phosphorus loading are driving the increase in blooms observed since 2010. 
The new 20-year fused cyanoHAB time-series allows for the evaluation or re-formation of predictive models in the face of changing environmental and meteorological drivers. While previous models have allowed for general predictions our analysis suggests that much variability is still unaccounted for and future predictions based on these simple models need to be used with caution. Stumpf et al. (2012) reported the first cyanoHAB prediction model that established an exponential relationship between Maumee River spring discharge and CI derived abundance from the 2002-2011 period. Sayers et al. (2016) found similar significant relationships between spring discharge and mean annual total and surface scum extents, respectively for the 2002-2013 period. This study found the 20-year time-series extent data to generally agree with the mean spring discharge models of Stumpf et al. (2012) and Sayers et al. (2016), however with greater deviations (and less predictive power) observed in larger outlier bloom years (2013 and 2015). This study also showed better prediction of extents using the weighted discharge metric suggested by Stumpf et al. (2016), however, there was still a significant amount of unexplained variance suggesting that spring discharge alone is of limited prediction power for very large bloom extents. Stumpf et al. (2016) discusses the unique set of conditions, including bloom initiation location and high turbidity, which possibly resulted in the large outlier bloom in 2015 that was not well predicted by discharge alone. This observation is in agreement with the finding of Sayers et al. (2016) that the annual frequency of sediment re-suspension events have some power to predict large cyanoHAB blooms.

Observed variance in the weighted discharge prediction of the 20 year time-series data is also possible due to the inherent difference in modeled metrics of annual cyanoHABs. The NOAA operational forecast (Stumpf et al. 2016) is built upon a predictive model using discharge to estimate cyanoHAB biomass (summation of individual image pixel CI values) and not areal extent where the new 20 year time-series data are estimates of areal extent regardless of biomass. The two metrics provide different information as there is the possibility to achieve a single basin-wide biomass value from either a spatially limited bloom of very high biomass or a large spatial bloom of low biomass. The new fused 
SeaWiFS/MODIS extent products provide a complementary dataset (to the NOAA CI product) to approach annual cyanoHAB predictions.

\subsection{Conclusions and Recommendations}

This new extended 20-year time series for the Western Basin of Lake Erie provides more quantitative support for the widespread observation that cyanoHABs have become more severe (larger and longer lasting) in recent years, beginning in the mid-2000s and accelerating after 2010. Comparison of the SeaWiFS and MODIS Aqua products at the pixel, daily, and annual scales confirm that the imagery from these two sensors was successfully fused into a single, consistent time series. Including SeaWiFS imagery from years prior to the launch of MODIS Aqua shows us that these earlier cyanoHAB events were smaller than in later years but spatially similar, with the most persistent and intense blooms located in Maumee Bay and along the southern shore of the basin with very little activity in the Detroit River outflow. Looking at the cumulative occurrence map for the 20year time series (Figure 5.8), only the plume of Detroit River water in the northwest corner of WBLE has consistently remained cyanoHAB-free.

This 20-year time series demonstrates the complexity of relating cyanoHABs extent and duration to drivers such as river discharge. The 20 -year record, which includes 8 -day seasonal composites, can be used to further quantify the effect of other drivers including meteorological conditions, resuspension events, land cover changes, and agricultural practices have on cyanoHABs extent and duration.

The MCH/SSI method could be further adapted for use with the CZCS imagery of Lake Erie collected from 1979-1987, providing better historical context for Lake Erie. This extends to the time period pre-mussel invasion and thus could allow for the quantification of the impact of these mussels on cyanoHAB events. The method could also be extended spatially, given that some Lake Erie cyanoHABs in recent years have extended into the central basin of the lake. Finally, the sediment plume maps incidentally produced as part 
of the MCH could be developed into their own time series to better understand variations in erosion and sediment load in the Maumee River.

\subsection{Acknowledgements}

Generation of recent MODIS cyanoHAB products was funded by the US Environmental Protection Agency (EPA), NOAA GLERL, and CIGLR under subcontract \#3004701270. Aspects of this study were also supported by the EPA under contract EP-R5-11-07 through subcontract \#427998 from Battelle Memorial Institute. The study was based on previous work supported by the EPA under grant GL-00E00855-0 as well as by the Great Lakes Observing System under contract \#3002475304 and The University of Michigan Water Center under contract \#3003032930. Aspects of this study were funded by the NASA Carbon Monitoring System under contract \#80NSSC17K0712. We thank Harry Stone and Stephanie Weber at Battelle for their technical feedback and review of the work on which this study was based. We would also like to acknowledge Beth Hinchey-Malloy and Frank Anscombe from EPA GLNPO for their technical contributions and encouragement. 


\section{Spatial and temporal variability of inherent and apparent optical properties in western Lake Erie: Implications for water quality remote sensing}

Lake Erie has experienced dramatic changes in water quality over the past several decades requiring extensive monitoring to assess effectiveness of adaptive management strategies. Remote sensing offers a unique potential to provide synoptic monitoring at daily time scales complementing in-situ sampling activities occurring in Lake Erie. Bio-optical remote sensing algorithms require knowledge about the inherent optical properties (IOPs) of the water for parameterization to produce robust water quality products. This study reports new IOP and apparent optical property (AOP) datasets for western Lake Erie that encapsulate the May-October period for 2015 and 2016 at weekly sampling intervals. Previously reported IOP and AOP observations have been temporally limited and have not assessed statistical differences between IOPs over spatial and temporal gradients. The objective of this study is to assess trends in IOPs over variable spatial and temporal scales. Large spatio-temporal variability in IOPs were observed between 2015 and 2016 likely due to the difference in the extent and duration of mid-summer cyanobacteria blooms. Differences in the seasonal trends of the specific phytoplankton absorption coefficient between 2015 and 2016 suggest differing algal assemblages between the years. Other IOP variables including colored dissolved organic matter (CDOM) and beam attenuation spectral slopes suggest variability is influenced by river discharge and sediment re-suspension. The datasets presented in this study show how these IOPs and AOPs change over a season and between years, and are useful in advancing the applicability and robustness of remote sensing methods to retrieve water quality information in western Lake Erie.

Keywords: Lake Erie; Harmful algal blooms; Inherent optical properties; Cyanobacteria; Remote sensing 


\subsection{Introduction and Background}

Over the past several decades the western basin of Lake Erie has experienced dramatic changes in water quality (Kane et al. 2014) that have negatively impacted the millions of people who rely on the lake for a source of drinking water, a place to recreate, and a location to run their businesses. Of particular concern are the annually recurring blooms of harmful cyanobacteria (cyanoHABs) that occur in the western basin, which in recent years are becoming more persistent and intense (Bridgeman et al. 2013, Michalak et al. 2013, Stumpf et al. 2012, 2016, Sayers et al. 2016, 2019 in this issue). Annual cyanoHAB occurrences have been linked to the annual spring discharge from the Maumee River (Stumpf et al. 2012 , 2016) which provides the large quantities of nutrients required to fuel these blooms. Long-term internal phosphorus loading of the basin has also recently been shown to play a role in annual cyanoHAB occurrence (Ho and Michalak 2017). Further complicating our understanding of bloom dynamics, is the massive abundance of filter feeding dreissenid mussels present in the western basin, which have been linked to increased cyanoHABs through selective feeding and nutrient cycling (Vanderploeg et al. 2001, Conroy et al. 2005, Smith et al. 2015). Fully understanding the combined effect of the many forcing functions on Lake Erie's water quality, more and better water quality measurements will be needed.

Traditional water quality monitoring in Lake Erie has been conducted using in situ and laboratory observations. The USEPA Great Lakes National Program Office (GLNPO) has conducted spring and summer water quality sampling on Lake Erie since the early 1980s (Barbiero et al. 2018) and the NOAA Great Lakes Environmental Research Laboratory (GLERL) has been conducting weekly monitoring for over five years. Many other universities and agencies also have monitoring programs producing valuable data sets. Even with the impressive number of assets making crucial measurements, it is likely not

possible to fully capture the spatial and temporal variability of various water quality parameters in Lake Erie with in situ data alone.

Satellite remote sensing offers a unique potential to provide synoptic water quality monitoring of large water bodies on a daily basis, which has proven to be extremely 
valuable to better understanding cause and effect relationships between observed phenomena and their forcing functions. Several investigators have developed or applied algorithms to retrieve water quality parameters in Lake Erie including chlorophyll-a (Binding et al. 2012, Shuchman et al. 2013, Lesht et al. 2013), water clarity (Binding et al. 2007, 2015), inorganic mineral concentrations (Binding et al. 2012, Shuchman et al. 2013), dissolved organic carbon, (Shuchman et al. 2013), and cyanobacteria presence (Vincent et al. 2004, Becker et al. 2009, Wynne et al. 2008, Sayers et al. 2016, Ho et al. 2017). These remote sensing products have provided valuable new insights and an ability to quantify broad changes in water quality in Lake Erie. While useful, these algorithms are limited in their ability to derive more sophisticated water quality information such as phytoplankton functional types (PFT) or species classifications. More advanced multi-spectral and hyperspectral algorithm methodologies with precise model parameterizations will be needed in order to accurately recover these more detailed water quality products.

Bio-optical algorithms, both empirical and semi-analytical, relate water quality properties to the spectral light field. In complex inland and coastal waters, bulk optical properties are controlled by the abundance and composition of the optically active constituents (OACs) which are comprised of phytoplankton, colored dissolved organic matter (CDOM), and non-algal particles (Werdell et al. 2018). The abundance of OACs are related to observed water leaving radiance, an apparent optical property (AOP), through their unique inherent optical properties (IOPs) which at the most fundamental level include spectral absorption and scattering (Morel and Prieur 1977). The IOP spectral shapes, which dictate the spectral shape of observed water leaving radiance, can be highly variable depending on the specific types of OACs that are in the water column (Moore et al. 2014). For example, different phytoplankton types can have distinctly different light absorbing pigment compositions that have distinct spectral shapes (Bricaud et al. 2004, Ciotti et al. 2002). As phytoplankton group composition changes over the course of a year or between years, so do the underlying IOPs. Remote sensing algorithms with the objective of identifying PFTs and other parameters must take into account the variability in spectral IOPs of the observed water body (IOCCG 2014, Mouw et al. 2017). 
Documented observations of IOPs in Lake Erie are relatively sparse in respect to their spatial and temporal variability. Binding et al. (2008) reported some of the first IOP measurements for Lake Erie by analyzing laboratory derived absorption coefficients for several months in 2004 and 2005. O'Donnell et al. (2010) made in situ IOP and AOP measurements for a two-day period in September 2007. Recently, Moore et al. (2017) reported a full suite of IOP and AOP observations for the western basin of Lake Erie acquired in mid-August for both 2013 and 2014 with the goal of characterizing the optical properties during the cyanobacteria bloom peak. While these measurements have been incredibly valuable for the development of remote sensing water quality retrieval algorithms, they are limited in their temporal scales. It is unclear from the presently reported IOP and AOP values how representative they are over the ice-free period and how consistent they are between years. Understanding the natural variability in IOPs over an entire season is important for remote sensing algorithms that require known values for algorithm parameterization. Semi-analytical algorithm frameworks reported by Binding et al. (2012), Shuchman et al. (2013), and Simis et al. (2005) all make assumptions about mass specific absorption and scattering coefficients computed as mean values from limited datasets. How these algorithms, using "default" parameterizations, perform over the vegetative season is unclear. Better understanding of the seasonal IOP and AOP variability will allow for the refinement of these approaches to provide more robust retrievals over long time periods.

In this study, we investigate the spatial and temporal variability of several IOP variables at weekly, monthly, and annual scales over the vegetative season. The aim of this study is to 1) present a new IOP and AOP dataset for Lake Erie at unprecedented temporal intervals (weekly); and 2) identify significant patterns and trends in IOPs and AOPs throughout the vegetative season. 


\subsection{Methods}

\subsubsection{Study Area}

Sampling was conducted on a near weekly basis in Lake Erie from May through October of 2015 and 2016, collecting data from 6 master stations (WE2, WE4, WE6, WE8, WE12, and WE13) shown on Figure 6.1. Station WE15 was sampled on a near weekly basis beginning in July 2015. Data was collected from two other stations but for less extensive time periods, and were not used in this analysis (WE9 was only sampled in 2016 and WE14 was only sampled from July through October of 2015). At each station instruments were deployed to measure IOPs/AOPs and water samples were collected for basic water quality measures, i.e., chlorophyll-a (Chl-a), phycocyanin (PC), colored dissolved organic material (CDOM), and total and fixed suspended solids (TSS and FSS, respectively). 


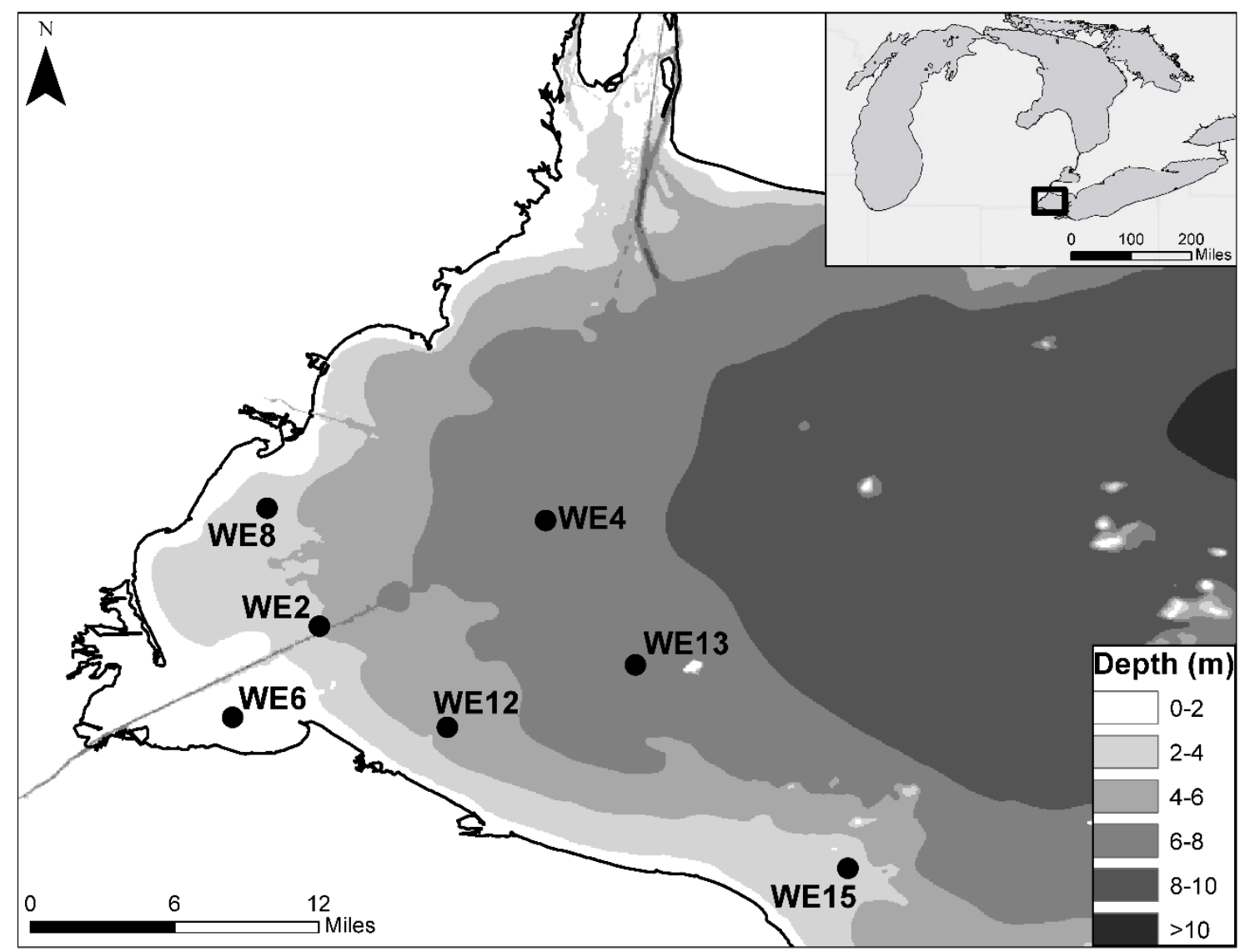

Figure 6.1. Station names and locations from 2015 and 2016 weekly data collections. Stations sampled regularly (weekly) are shown as black dots. Basin bathymetry is shown as varying shades of gray with darker colors indicating deeper water depth.

\subsubsection{Biogeochemical / Laboratory Data}

Water samples from the top meter of the water column were collected with a Niskin bottle and poured into three sample bottles simultaneously, repeating until sample bottles were full. Samples for pigment analyses were filtered onto Whatman GF/F filters, extracted with appropriate solvent, and analyzed fluorometrically. For Chl-a samples, filters were extracted with N, N-dimethylformamide (Speziale et al., 1984) and analyzed on a Turner Designs fluorometer calibrated with Chl-a a standards. Filters for PC determination were 
extracted in phosphate buffer (Ricca Chemical, $\mathrm{pH}$ 6.8) using two freeze-thaw cycles, followed by sonication (Horváth et al., 2013). Relative fluorescence was measured on a Turner Aquafluor fluorometer and converted to PC concentration using a series of dilutions of a commercial standard (Sigma-Aldrich). The concentration of total suspended solids (TSS) was determined gravimetrically after filtering the sample through a pre-dried, preweighed Whatman GFC 47-mm filter. The filter was then combusted for 4 hours at $450^{\circ} \mathrm{C}$, cooled, and reweighed to measure volatile suspended solids (VSS; APHA, 1998). The concentration of fixed suspended solids (FSS), the non-organic sediments in the water sample, was determined as the difference between TSS and VSS concentrations. Lake water was filtered under low vacuum on pre-rinsed $(1 \mathrm{~N} \mathrm{HCl}) 0.2 \mu \mathrm{m}$ pore size Millipore membranes for CDOM absorption measurements and refrigerated $\left(4^{\circ} \mathrm{C}\right)$ in the dark until analyzed. Absorbance of the filtered water was measured in a $10 \mathrm{~cm}$ quartz cuvette from 400 to $700 \mathrm{~nm}$ at $2 \mathrm{~nm}$ increments with a dual beam spectrophotometer (Perkin Elmer, lambda 40), with deionized water used as the reference (Effler et al., 2010). CDOM spectral slope $(\mathrm{S})$ was calculated using a non-linear exponential fit over the wavelength range from 400 to $500 \mathrm{~nm}$ (Twardowski et al., 2004) using MATLAB code provided by the Maine InSitu Sound \& Color Lab (http://misclab.umeoce.maine.edu).

\subsubsection{Optical Data}

IOP data were collected using a WET Labs spectral absorption and attenuation meter (acs) (WET Labs, Inc.). This meter was deployed over the side of the research vessel, along with a CTD instrument (Sea-Bird Scientific) to record temperature and depth, and lowered to just above the lake floor, collecting data throughout the water column. The ac-s measured spectral attenuation and absorption at 86 wavelengths from approximately 400 $\mathrm{nm}$ to $740 \mathrm{~nm}$. In-lab calibration using purified Milli-Q water was conducted according to WET Labs instructions on a regular basis between field cruises in order to track instrument drift (https://www.seabird.com/transmissometers/ac-s-spectral-absorption-andattenuation-sensor/). 
A Satlantic HyperPro II profiler (Satlantic, Inc.) was deployed at the stations to collect upwelling radiance $\left(\mathrm{L}_{\mathrm{u}}\right)$ and downwelling irradiance $\left(\mathrm{E}_{\mathrm{d}}\right)$ throughout the water column. The profiler was deployed off the side of the research vessel and collected data while slowly free-falling through the water column. ProSoft software (v7.7, Satlantic, Inc.) was used to process the HyperPro data for analysis, binning data to 0.3 meter depth intervals. Processing the raw data files to level 4 generated diffuse attenuation coefficients through the water column (Sea-Bird Scientific, 2017). Apparent optical depth, the depth where 90\% of water leaving radiance originates (Gordon and McCluney 1975), was calculated as 1.3 divided by the wavelength-specific attenuation coefficients derived from the profiler's $E_{d}$ sensor (Werdell and Bailey 2005; Bailey and Werdell, 2006).

Hyperspectral above-water radiance was collected using a Satlantic HyperGun (Satlantic, Inc.). This instrument collects radiance at 137 channels covering a spectral range of approximately 350-800 $\mathrm{nm}$ with an approximate spectral resolution of $3 \mathrm{~nm}$ and a 3-degree field of view. Upwelling radiance $\left(\mathrm{L}_{\mathrm{u}}, \mathrm{W} / \mathrm{m}^{2} / \mathrm{sr}^{-1} / \mathrm{nm}^{-1}\right)$ was measured at approximately 150 degrees relative to the solar azimuth at the time of measurement (Mobley, 1999). The HyperGun was pointed at the water's surface at 40 degrees from nadir and measurements were taken for 15 seconds. The HyperGun was shifted 90 degrees upward ( 40 degrees from zenith) and radiance measurements were taken of the sky, $\mathrm{L}_{\text {sky }}$, for approximately 15 seconds. The measured radiance of an $18 \%$ reflective panel was collected at approximately 40 degrees from nadir for approximately 15 seconds. Additionally, care was taken to avoid shadows, sun glint, and floating debris when collecting data. HyperGun data were downloaded from the instrument, radiometrically calibrated and dark-offset corrected using the most recent factory calibration file. Irradiance $\left(\mathrm{E}_{\mathrm{d}}, \mathrm{W} / \mathrm{m}^{2} / \mathrm{nm}^{-1}\right)$ was calculated as the radiance of the panel divided by the known reflectance of the panel (0.18) and multiplied by $\pi$ (Mobley, 1999; Mueller et al., 2003). The water leaving radiance $\left(\mathrm{L}_{\mathrm{w}}\right)$ was corrected for diffuse sky contamination using the following equation: $\mathrm{L}_{\mathrm{w}}=\mathrm{L}_{\mathrm{u}}-0.028 * \mathrm{~L}_{\text {sky }}$ (Mobley, 1999), where 0.028 is taken to be the reflectivity of the water's surface. The remote sensing reflectance $\left(\mathrm{R}_{\mathrm{rs}}(\lambda), \mathrm{sr}^{-1}\right)$ was calculated as $\mathrm{L}_{\mathrm{w}}$ divided by $\mathrm{E}_{\mathrm{d}}$. A SavitskyGolay filter (Savitsky and Golay, 1964) was used to smooth the noise in the spectra. 
Raw data from the ac-s instrument was processed through the WET Labs Archive Processing (WAP) tool (v4.37, WET Labs, Inc.). Outlier calibration spectra caused by bubbles in the water supply were removed, the average calibration absorption and attenuation spectra were calculated, and temperature corrected. Field data from the ac-s and CTD instruments were merged, correcting for time lags. The ac-s absorption and attenuation data were interpolated to common wavelengths (WET Labs Inc., 2011). Absorption tube scattering corrections were applied to the ac-s data to account for overestimations of the absorption coefficients due to uncollected scattered light (Rottgers et al. 2013). Temperature corrections were applied based on the temperature retrieved from the CTD instrument (Sullivan et al., 2006) and instrument drift corrections were applied based on the most recent instrument pure water calibration spectra. Additional checks were performed on ac-s data, resulting in the removal of data meeting any of the following criteria: absorption is greater than attenuation, absorption or attenuation less than -0.005 (due to instrument precision, negative data values greater than -0.005 were set to zero), or saturated backscattering data (https://www.seabird.com/transmissometers/ac-s-spectralabsorption-and-attenuation-sensor/). All remaining data were binned by depth at intervals of 0.1 meters.

After initial processing of the field data, additional steps were taken to prepare the data for final analysis. Hyperspectral IOP data were aggregated over the apparent optical depth from each station to best represent the water that was visible to satellite imaging sensors. If no HyperPro profiler data were available on a given date, the season-long average apparent optical depth for that station was used. The season-long average was used in place of the most recent sampling because a visual inspection of the optical depth spectra through the season revealed substantial variability from week to week. A minimum depth of 1 meter was used to ensure that enough field data was included in the aggregated output. Station WE6 was too shallow to calculate attenuation, resulting in no apparent optical depth data. For this station, all IOP data were aggregated over the top 1 meter. After apparent optical depth aggregation, data were removed that failed a few basic checks: if the absorption or attenuation values were greater in red $(640 \mathrm{~nm})$ than in blue $(420 \mathrm{~nm})$ or if the ratio of 
absorption or attenuation at $500 \mathrm{~nm}$ to $440 \mathrm{~nm}$ was less than 1 . A Savitsky-Golay filter was used to smooth noise in the spectral curves (Savitsky and Golay, 1964). In the approximately $7 \%$ of cases where the beam attenuation spectral curves did not follow the expected exponential decay trend from $400-420 \mathrm{~nm}$, the curve fit function in Python's SciPy module was used to correct the shape of the curve (Jones et al., 2001). All optical data are available by request from the NOAA Great Lakes Environmental Research Laboratory (GLERL).

\subsection{Data Analysis}

Scattering (b) was derived from the ac-s meter-generated absorption (a) and beam attenuation (c) data according to equation 1 :

$$
\text { Equation 6.1: } c(\lambda)=a(\lambda)+b(\lambda)
$$

Absorption can be partitioned into constituent components according to equation 2:

$$
\text { Equation 6.2: } a_{t}(\lambda)=a_{w}(\lambda)+a_{p g}(\lambda)
$$

where $\mathrm{a}_{\mathrm{w}}$ is the absorption due to pure water and $\mathrm{a}_{\mathrm{pg}}$ is the absorption due to particulates, $a_{p}$, plus the absorption due to CDOM, ag. The output from the ac-s has already had pure water absorption removed (WET Labs Inc., 2013), leaving only the combined particulate and CDOM absorption as the constituent parts in the spectra. Using the lab-derived CDOM absorption at $400 \mathrm{~nm}$ (асдом400) and the calculated CDOM spectral slope ( CDOM absorption spectra were derived according to equation 3 (Twardowski et al. 2004):

$$
\text { Equation 6.3: } \mathrm{a}_{\operatorname{CDOM}} \lambda=\mathrm{a}_{\mathrm{CDOM}} 400 * \mathrm{e}^{-\mathrm{S}(\lambda-400)}
$$

Subtracting the CDOM absorption spectra from the $a_{p g}$ spectra results in the particulate absorption spectra $\left(\mathrm{a}_{\mathrm{p}}(\lambda)\right)$, which is the absorption due to algal and non-algal particles in the water. Particulate beam attenuation $\left(c_{p}(\lambda)\right)$ can then be derived according to Equation 4: 


$$
\text { Equation 6.4: } c_{p}(\lambda)=a_{p}(\lambda)+b_{p}(\lambda)
$$

Where $b_{p}$ is the scattering coefficient derived from eq.1. Since CDOM scattering is assumed negligible or non-existent and pure water scattering has already been removed, the calculated total scattering is inherently due to in water particulates.

Several IOP optical proxies were generated from the derived $a_{p}$ spectra. The particulate absorption $\mathrm{PC}$ line height $\left(\mathrm{PCLH}_{\mathrm{ap}}\right)$ was used as a proxy for the presence of $\mathrm{PC}$ and was derived using a variation of the absorption line height methods described in Roesler and Barnard (2013). $\mathrm{PCLH}_{\mathrm{ap}}$ was calculated as the difference between $\mathrm{a}_{\mathrm{p}}$ at $620 \mathrm{~nm}$ (the PC absorption peak; Simis et al., 2005, Roy et al. 2011) and the baseline absorption at $620 \mathrm{~nm}$. The mass specific phytoplankton absorption coefficient at $665 \mathrm{~nm}\left(\mathrm{a}_{\mathrm{ph}} 665\right)$ was used as a proxy for phytoplankton size/type and was derived as the particulate absorption chlorophyll line height (CHLLH - described below) divided by the corresponding lab-derived Chl-a concentration for each sample. The absorption at $665 \mathrm{~nm}$ was used instead of $676 \mathrm{~nm}$ as used by Slade and Boss (2015) because several relevant remote sensing algorithms including those proposed by Simis et al. (2005) and Binding et al. (2012) use specific phytoplankton absorption around this band in their respective bio-optical models, which were developed in cyanoHAB conditions.

The absorption line height approach used in the calculation of $\mathrm{PCLH}_{\mathrm{ap}}$ and $\mathrm{a}{ }_{\mathrm{ph}} 665$ is fully described in Roesler and Barnard (2013) as well as in Slade and Boss (2015). Briefly, the absorption line height method calculates the difference of observed particulate absorption at a reference wavelength above a baseline absorption value. This approach allows for the estimation of pigment absorption in the presence of other absorbing constituents, which in the case of $a_{p}$ are other non-algal particles. The baseline absorption value approximates the absorption from non-algal particles and is derived using a linear equation of the form:

$$
\text { Equation 6.5: } a_{B L}\left(\lambda_{\text {ref }}\right)=\frac{a\left(\lambda^{+}\right)-a\left(\lambda^{-}\right)}{\lambda^{+}-\lambda^{-}} *\left(\lambda_{\text {ref }}-\lambda^{-}\right)+a\left(\lambda^{-}\right)
$$


Where $\lambda_{\text {ref }}$ is the reference wavelength ( $620 \mathrm{~nm}$ for $\mathrm{PCLH}_{\mathrm{ap}}$ and $665 \mathrm{~nm}$ for CHLLH), $\lambda^{-}$ is the wavelength at baseline start ( $600 \mathrm{~nm}$ for PCLH $\mathrm{Pap}_{a}$ and $650 \mathrm{~nm}$ for CHLLH), and $\lambda^{+}$is the wavelength at baseline end (648 nm for PCLHap and $715 \mathrm{~nm}$ for CHLLH).

The line height absorption is then calculated as:

$$
\text { Equation 6.6: } a_{L H}\left(\lambda_{\text {ref }}\right)=a\left(\lambda_{\text {ref }}\right)-a_{B L}\left(\lambda_{\text {ref }}\right)
$$

The particulate beam attenuation spectral slope $(\gamma)$ was calculated using a non-linear power law fit over the wavelength range from 400 to $500 \mathrm{~nm}$ (Boss et al., 2001; Slade and Boss, 2015) using MATLAB code provided by the Maine In-Situ Sound \& Color Lab (http://misclab.umeoce.maine.edu). The particulate beam attenuation spectral slope represents the variability in particle size distributions (Boss et al. 2001).

\subsection{Results}

Large variations in water constituents (Chl-a, PC, FSS) were observed over the course of the two-year study period. Table 6.1 summarizes the range, mean, and median Chl-a, PC, and FSS values for 2015, 2016, and combined for both years. The table represents data from all sites with the exception of one sample from station WE8 on August 10, 2015 due to extremely large concentrations of surface scum (e.g. $6784 \mathrm{mg} / \mathrm{m}^{3} \mathrm{Chl}-\mathrm{a}$ and $8228 \mathrm{mg} / \mathrm{m}^{3}$ PC). Additionally the optical proxy values are also summarized on the table and are described in detail in subsequent sections.

Table 6.1. Summary statistics for the four inherent optical properties (aph*665, PCLHap, SCDOM, $\gamma$ ) and laboratory biochemical parameters (Chl-a, PC, and FSS). Summary statistics include range, mean, and median values for each year and combined for both years. 


\begin{tabular}{|c|c|c|c|c|c|c|c|c|c|c|}
\hline \multirow[b]{2}{*}{ Parameter } & \multirow[b]{2}{*}{ Units } & \multicolumn{3}{|c|}{2015} & \multicolumn{3}{|c|}{2016} & \multicolumn{3}{|c|}{ Combined } \\
\hline & & Range & Mean & Median & Range & Mean & Median & Range & Mean & Median \\
\hline$a_{p h}{ }^{*} 665$ & $\mathrm{~m}^{2} / \mathrm{mg}^{-1}$ & $<0.001-0.015$ & 0.003 & 0.003 & $<0.001-0.009$ & 0.004 & 0.004 & $<0.001-0.015$ & 0.004 & 0.003 \\
\hline $\mathrm{PCLH}_{\mathrm{ap}}$ & $\mathrm{m}^{-1}$ & $<0.001-0.130$ & 0.017 & 0.010 & $<0.001-0.11$ & 0.008 & 0.003 & $<0.001-0.13$ & 0.012 & 0.006 \\
\hline $\mathrm{S}_{\mathrm{CDOM}}$ & $\mathrm{nm}^{-1}$ & $0.014-0.020$ & 0.017 & 0.017 & $0.011-0.026$ & 0.017 & 0.018 & $0.011-0.026$ & 0.017 & 0.017 \\
\hline y & $\mathrm{nm}^{-2}$ & $0.21-2.05$ & 0.91 & 0.84 & $0.49-2.26$ & 1.01 & 1.01 & $0.21-2.26$ & 0.96 & 0.93 \\
\hline Chl-a & $\mathrm{mg} / \mathrm{m}^{3}$ & $1.01-352.60$ & 43.95 & 28.32 & $1.27-114.6$ & 15.63 & 7.95 & $1.01-352.6$ & 29.84 & 12.55 \\
\hline PC & $\mathrm{mg} / \mathrm{m}^{3}$ & $0.00-514.20$ & 26.07 & 9.45 & $0.01-42.24$ & 2.62 & 0.66 & $0.0-514.2$ & 14.25 & 1.43 \\
\hline FSS & $\mathrm{g} / \mathrm{m}^{3}$ & $1.07-129.60$ & 12.97 & 7.24 & $0.28-51.88$ & 8.40 & 6.28 & $0.28-129.6$ & 10.75 & 6.43 \\
\hline
\end{tabular}

Values of Chl-a, PC, and FSS were different between 2015 and 2016 (Fig. 6.2). Median Chl-a values in August 2015 were more than double those in August 2016. Higher median Chl-a was also observed in 2015 for July and September while similar values were observed between years in May and October (Fig 6.2 top panel). Much larger PC values were observed in July, August, and September 2015 compared to values in 2016 (Fig. 6.2 middle panel). Lastly, FSS was highly variable for both years with the largest values in 2015 occurring in June and October while for 2016 the largest values occurred from August-October (Fig 6.2 bottom panel).
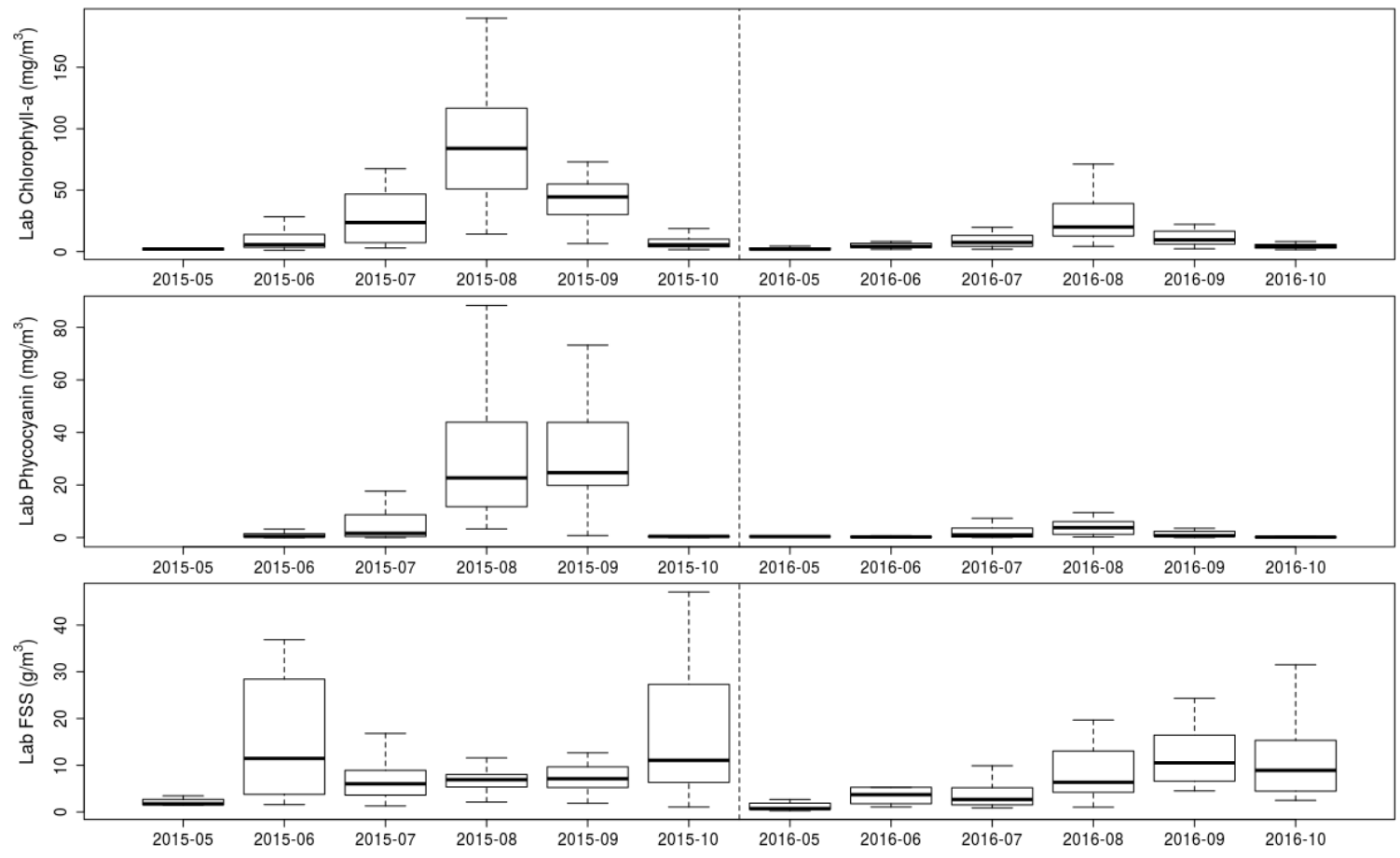

Figure 6.2. Temporal patterns of chlorophyll-a (Top panel), phycocyanin (middle panel), and fixed suspended solids (bottom panel) in western Lake Erie 
expressed as boxplots where the black horizontal line is the median, the bottom and top of the box are the first and third quartiles respectively, and the horizontal gray lines below and above the box are the minimum and maximum values respectively. Monthly median values for 2015 (left half) and 2016 (right half) are shown in all three panels.

Mean monthly IOP parameter (absorption, scattering, and beam attenuation) magnitudes displayed a wide range in variability for WBLE in 2015 and 2016 (Fig. 3 left panels). For example, IOP magnitudes were quite similar for August in both 2015 and 2016 while magnitude differences between October 2015 (low magnitude) and 2016 (high magnitude) were very large. In fact, the magnitudes in October 2015 and 2016 represent the minimum and maximum mean monthly IOP values for both years combined. Even with the large differences observed in October, magnitudes in all three IOPs were generally higher in 2015 (solid lines) than 2016 (dashed lines).

Spectral differences were observed for all three IOP parameters (Fig. 3 right panels) by month and year with the largest differences occurring between October 2015 and 2016 again spanning the range of all other months and years. The largest differences occur in the blue wavelengths ( $400-450 \mathrm{~nm}$ ) where the decreasing slopes vary by month and year. Conversely, the normalized absorption curves (Fig. 3 right middle panel) for September 2015 and 2016 are nearly identical, both of which exhibit a peak at the Chl-a absorption feature at approximately $665 \mathrm{~nm}$. This feature also appears in both August 2015 and 2016 coinciding with cyanoHAB bloom events. However, very little visual evidence of the Chla absorption feature at $665 \mathrm{~nm}$ exists in October 2015 and 2016 or in July 2015 indicating strong absorption by CDOM or non-algal particles relative to phytoplankton absorption in this wavelength region. The normalized spectral scattering coefficients (Fig. 3 right bottom panel) display two distinct groups of spectral shapes with September 2016 and October 2016 very similar to each other and yet quite different from the other months and years. 

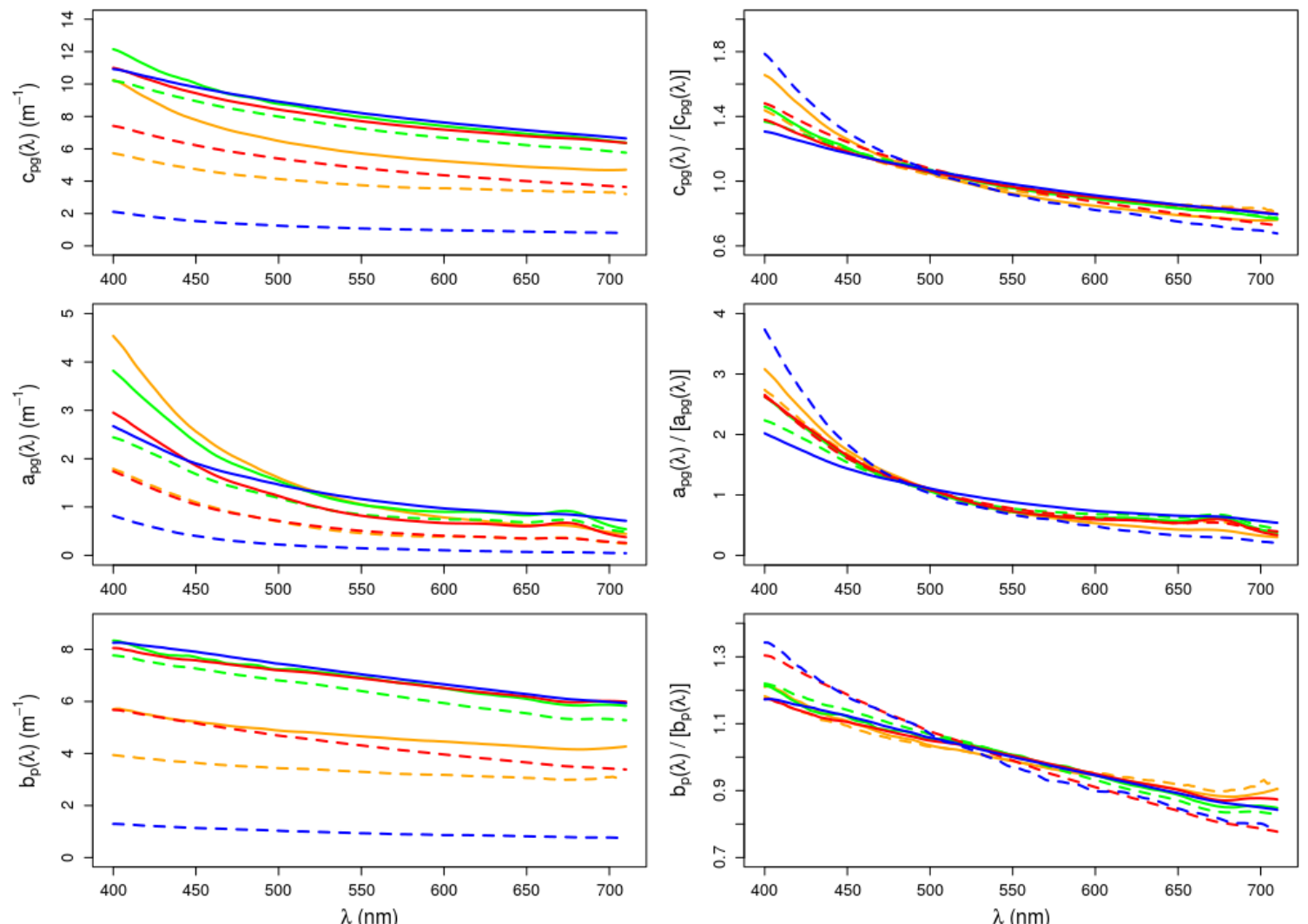

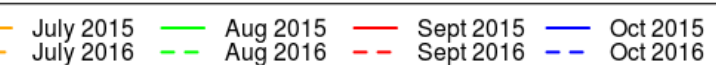

Figure 6.3. Monthly mean beam attenuation, absorption, and scattering coefficients for 2015 and 2016. Absolute values are shown in the left panels while mean normalized spectra are shown in the right panels. Lines are colored by month where solid lines represent 2015 data and dashed lines 2016 data. Note absolute value spectra (left panels) are on different y-axis scales.

Spatial variability in mean IOP magnitude (Fig. 6.4 left panels) was observed between five stations in the WBLE 2015-2016 dataset. The maximum values for all three parameters were observed at WE12 followed closely by WE2. Of the regularly sampled stations, these two stations were in closest proximity to the mouth of the Maumee River. A distinct separation in beam attenuation and scattering between stations WE12 and WE2 and all other stations was evident. Station WE4 exhibited the lowest absorption for all spectral 
regions and lowest beam attenuation in the $\sim 400-500 \mathrm{~nm}$ range. In contrast, WE8 displays the lowest scattering but relatively large absorption values.

Spatial differences in shape are clearly observed in the normalized IOP spectra (Fig. 6.4 right panels) between the five stations in the 2015-2016 dataset. Stations WE4 and WE13 exhibited nearly identical beam attenuation spectra (Fig. 4 right top panel) where as WE2, WE12, and WE8 were different. The normalized absorption spectra (Fig. 4 right middle panel) for stations WE2 and WE8 were identical while the remaining stations exhibit slight shape differences in the short blue $(400-450 \mathrm{~nm})$ spectral region. The Chl-a absorption feature at $665 \mathrm{~nm}$ was observed at all stations with slight differences in shape. The PC absorption peak at $620 \mathrm{~nm}$ was also observable at all stations. Two distinct groupings were evident in the normalized spectral scattering (Fig. 6.4 right bottom panel) spectra with stations WE2, WE12, and WE8 showing very similar shape while WE4 and WE13 were similar. The two groups exhibited clearly different spectral scattering slopes. 

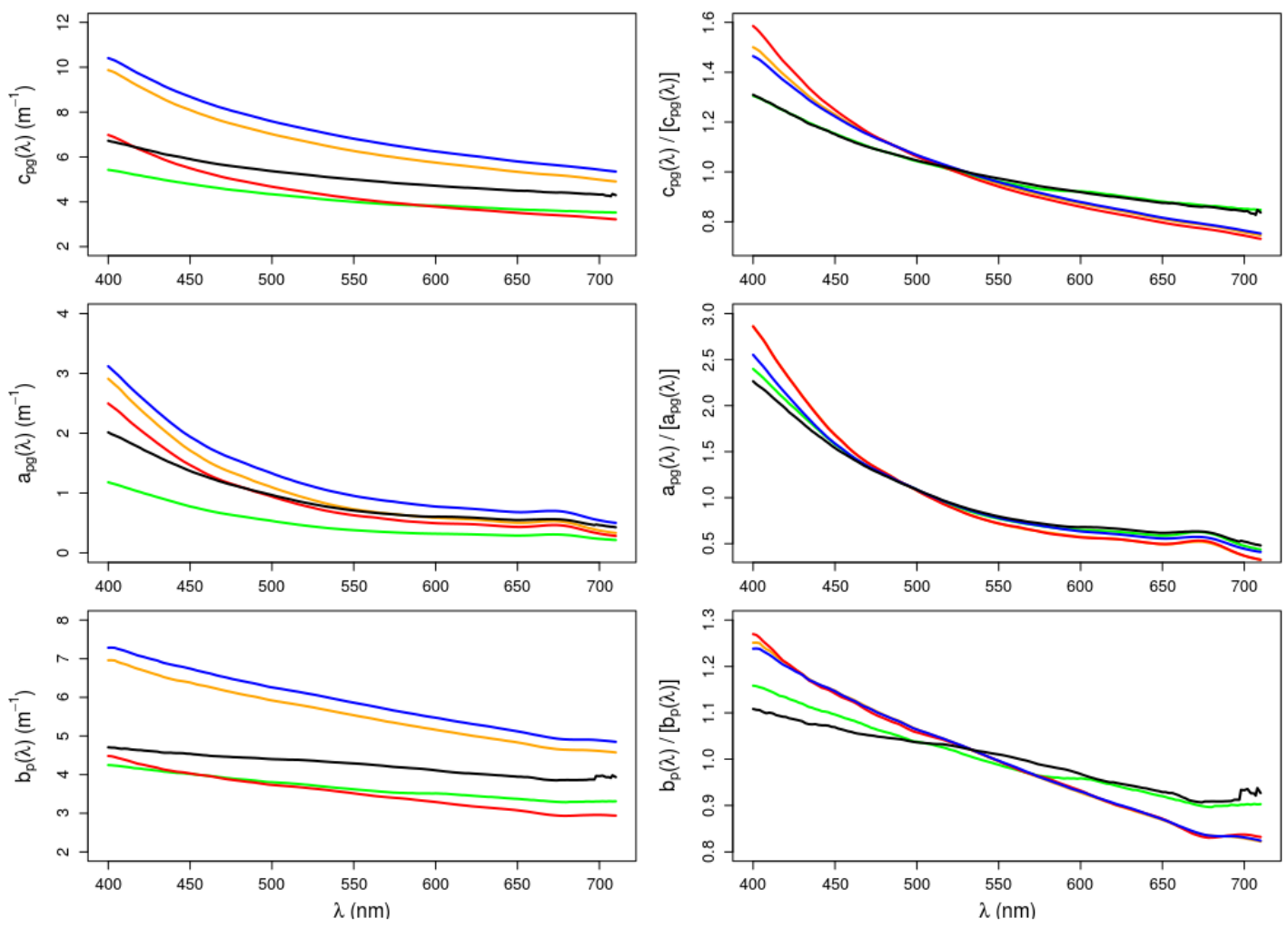

- WE2 — WE4 — WE8 — WE12 — WE13

Figure 6.4. Station mean (2015 and 2016 combined) beam attenuation, absorption, and scattering coefficients. Absolute values are shown in the left panels while mean normalized spectra are shown in the right panels. Lines are colored by station location. Note absolute value spectra (left panels) are on different y-axis scales.

The mass specific phytoplankton absorption coefficient at $665 \mathrm{~nm}, \mathrm{a}_{\mathrm{ph}} * 665$, was analyzed as a proxy for phytoplankton/algal community (i.e. size) variability which displayed seasonal and annual differences in median values (Fig. 5 top panel). The mean, median, and range of $a_{\mathrm{ph}} * 665$ values are displayed in table 1. Using the Mann Whitney $U$ test (Mann and Whitney, 1947), significant differences from the overall median $\mathrm{a}_{\mathrm{ph}} * 665$ values were observed in 2015 for May $(p<0.001)$, August $(p<0.001)$, September $(p-0.024)$, and October $(p<0.001)$. Significant differences from the overall median were also identified in 
2016 for May $(\mathrm{p}=0.001)$, June $(\mathrm{p}=0.008)$, September $(\mathrm{p}=0.006)$, and October $(\mathrm{p}=0.021)$. The largest $\mathrm{a}_{\mathrm{ph}} * 665$ values for both 2015 and 2016 were observed in May and exhibited steady declines through August as the phytoplankton community shifts from predominantly diatoms to colonial cyanobacteria (Bosse et al. 2019, this issue). While May $\mathrm{a}_{\mathrm{ph}} * 665$ were largest for both years, they were significantly different from each other $(\mathrm{p}=0.016)$ with greater median values occurring in 2015 . For $2015, \mathrm{a}_{\mathrm{ph}} * 665$ values increased from August through October while in 2016 the values continue to decrease from August through October. The difference in seasonal trends between years is a result of significantly different values of $\mathrm{a}_{\mathrm{ph}} * 665$ in August (2015 lower than 2016, $\left.\mathrm{p}<0.001\right)$ and October (2015 greater than $2016, \mathrm{p}=0.004)$ respectively. Large variability in monthly $\mathrm{a}_{\mathrm{ph}}$ *665 values are observed in both 2015 and 2016, however, the variability is not consistent between years. For example, large variability $\mathrm{a}_{\mathrm{ph}} * 665$ was observed in June 2015 while there was little variability in June 2016.

There were no significant differences in combined study (all 2015 and 2016 data) median $\mathrm{a}_{\mathrm{ph}}$ *665 values between sampling stations (Fig. 6.5 middle panel). Stations also exhibited very similar variability in $\mathrm{a}_{\mathrm{ph}} * 665$ with the exception of WE4 which displayed a larger degree of variance relative to other stations. Additionally, all stations exhibited median values similar to the combined study period median value $\left(0.003 \mathrm{~m}^{2} / \mathrm{mg}^{-1}\right)$. Two stations, WE12 and WE15, displayed significant differences (WE12 p=0.05, WE15 p=0.02) in $\mathrm{a}_{\mathrm{ph}}$ *665 between 2015 and 2016 while all others were not significantly different between years (Fig. 6.5 bottom panel). However, with the exception of WE6, all stations exhibited greater median values in 2016 compared with 2015 . Annual variability in $\mathrm{a}_{\mathrm{ph}}{ }^{*} 665$ for each station was inconsistent as some stations showed similar variability between 2015 and 2016 (e.g. WE15) while others (e.g. WE6) were very different between years. Finally, there were no significant differences between sampling station annual median $\mathrm{a}_{\mathrm{ph}}{ }^{*} 665$ values and the overall combined median value. 

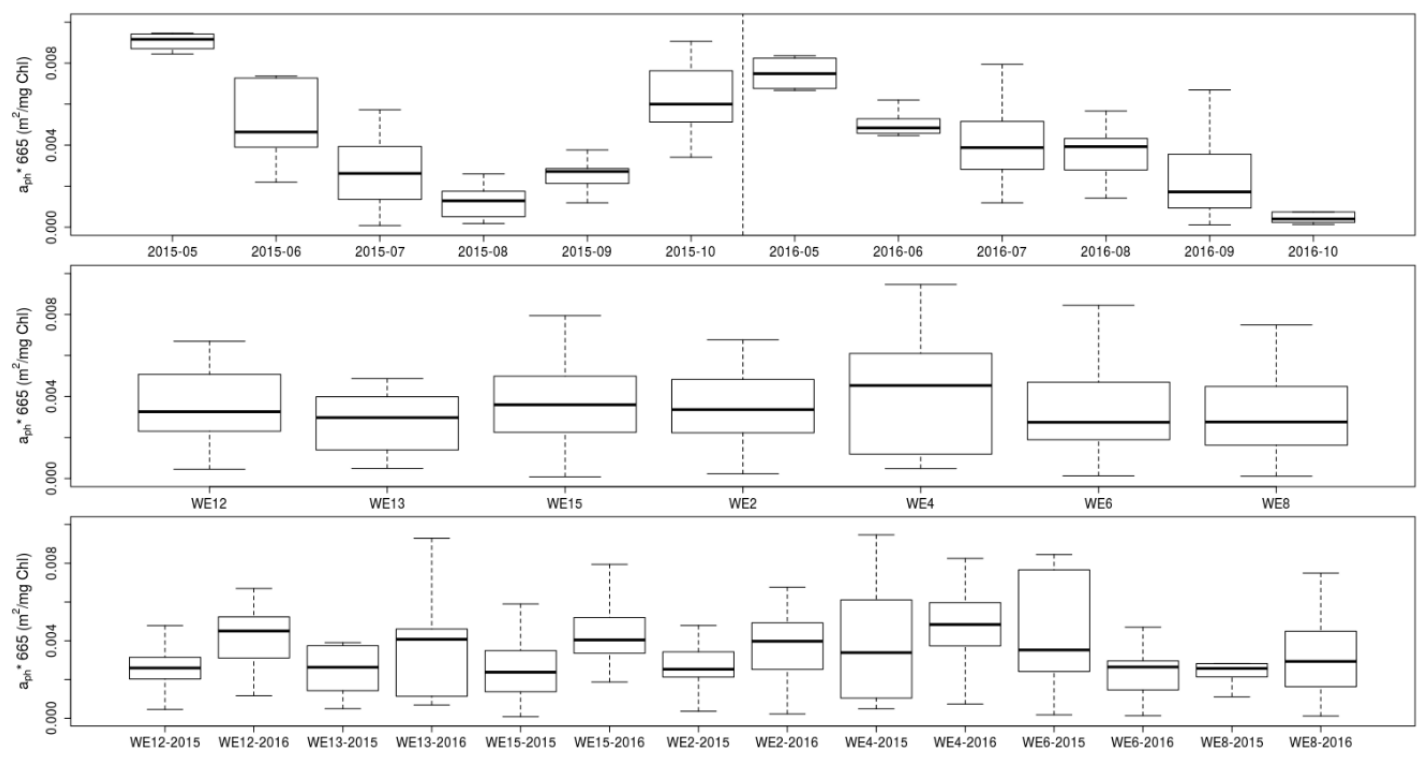

Figure 6.5. Spatial and temporal patterns of $a_{p h} * 665$ in western Lake Erie expressed as boxplots where the black horizontal line is the median, the bottom and top of the box are the first and third quartiles respectively, and the horizontal gray lines below and above the box are the minimum and maximum values respectively. Monthly median values for 2015 (left half) and 2016 (right half) are shown in the top panel. Station median values for the combined 20152016 dataset are shown in the middle panel. Annual station median values are shown in the bottom panel.

The particulate absorption PC line height $\left(\mathrm{PCLH}_{\mathrm{ap}}\right)$ was used to examine temporal and spatial variability of PC absorption as a proxy for cyanobacteria abundance (Fig. 6.6). Comparisons between $\mathrm{PCLH}_{\mathrm{ap}}$ values and coincident PC extracted concentrations revealed a strong positive relationship (Pearson's correlation $=0.72)\left(\right.$ Fig. 6.6). While PCLH $_{\text {ap }}$ and PC concentration were positively correlated, a high degree of variability was also observed, indicating $\mathrm{PCLH}_{\mathrm{ap}}$ may be sensitive to other absorbing pigments. For example, Chl-a exhibits a slight absorption feature in the same spectral region $(\sim 620 \mathrm{~nm})$ as PC absorption (Roy et al. 2011) which contributes to the $\mathrm{PCLH}_{\mathrm{ap}}$ in addition to PC. It also should be noted that the spread observed in figure 6 could also be an effect of pigment packaging where the 
$\mathrm{PCLH}_{\mathrm{ap}}$ was derived from particulate absorption where pigments remain bound in the cells/colonies while the laboratory PC has been extracted as is measured in solution.

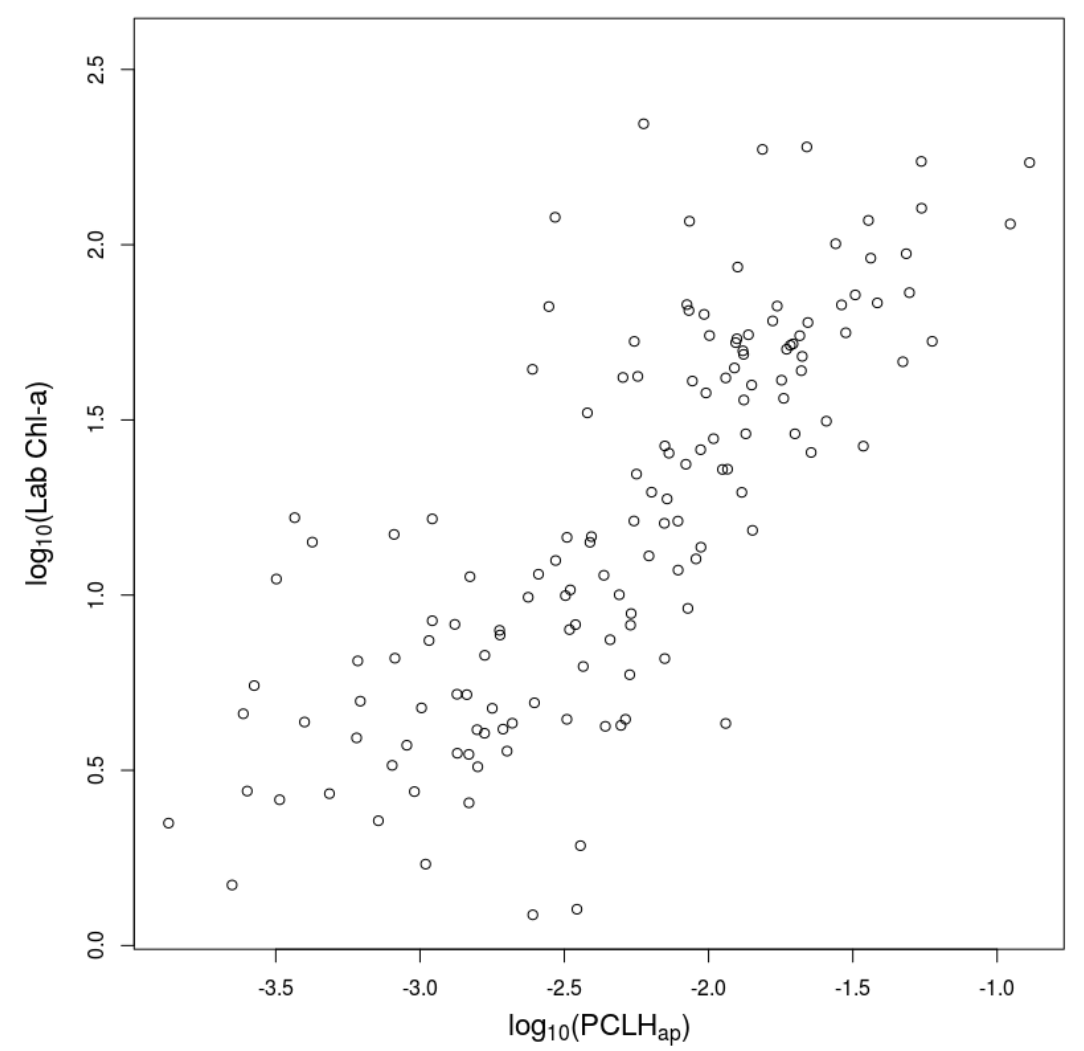

Figure 6.6. Comparison between particulate absorption phycocyanin line height $\left(\mathrm{PCLH}_{\mathrm{ap}}\right)$ (x-axis) and laboratory extracted phycocyanin concentration (Lab PC)(y-axis). Note the $\log _{10}$ scales.

The mean, median, and range of $\mathrm{PCLH}_{\mathrm{ap}}$ values are also displayed in table 1. Significant differences (see figure 7) from the overall study median $\mathrm{PCLH}_{\mathrm{ap}}$ value $\left(0.006 \mathrm{~m}^{-1}\right)$ were observed in 2015 for May ( $\mathrm{p}=0.005)$, August $(\mathrm{p}<0.001)$, September $(\mathrm{p}<0.001)$, and October $(\mathrm{p}=0.02)$, while differences were also identified in 2016 for June $(\mathrm{p}=0.004)$, September $(\mathrm{p}=0.009)$, and October $(\mathrm{p}=0.011)$. The monthly trends in $\mathrm{PCLH}_{\mathrm{ap}}$ values were generally consistent between 2015 and 2016 with both years showing low values in May increasing through August followed by a steady decline through October. Additionally, the highest maximum values for 2015 and 2016 were observed in August coinciding with the peak 
cyanoHAB period (Wynne et al. 2015, Sayers et al. 2019 in this issue). While the seasonal trends were similar, the degree of $\mathrm{PCLH}_{\mathrm{ap}}$ increase from May to August was larger in 2015 than in 2016 indicating much greater abundance of cyanobacteria presence in 2015 than 2016. This is confirmed through a comparison of monthly median $\mathrm{PCLH}_{\mathrm{ap}}$ values between 2015 and 2016, where both August and September 2015 values were significantly greater than the 2016 August and September PCLHap values (August p=0.013; September $\mathrm{p}<0.001)$.

Spatial variability in the combined two-year study period sample station median PCLH $_{a p}$ values were observed (Fig. 6.7 middle panel), with station WE4 exhibiting significantly lower median PCLH $\mathrm{Pp}_{\mathrm{ap}}$ values than WE6 (p<0.001). Stations WE12, WE13, WE15, WE2, WE6, and WE8 did not have significantly different $\mathrm{PCLH}_{\mathrm{ap}}$ values over the two-year period. Moreover, all stations with the exception of WE4 showed comparable PCLH $_{a p}$ variability over the two-year study period. Additionally, the median PCLH $_{a p}$ value for WE4 was significantly smaller $(\mathrm{p}=0.008)$ than the overall study median value while WE6 was significantly greater $(\mathrm{p}<0.001)$. There were also significant differences in median annual $\mathrm{PCLH}_{\mathrm{ap}}$ values for stations between 2015 and 2016 (Fig. 6.7 bottom panel). For example, stations WE12 ( $\mathrm{p}=0.008), \mathrm{WE} 15(\mathrm{p}=0.012)$, and WE2 $(\mathrm{p}=0.003)$ all had significantly lower $\mathrm{PCLH}_{\mathrm{ap}}$ values in 2016 than in 2015, while WE13, WE4, WE6, and WE8 had no difference between years. 

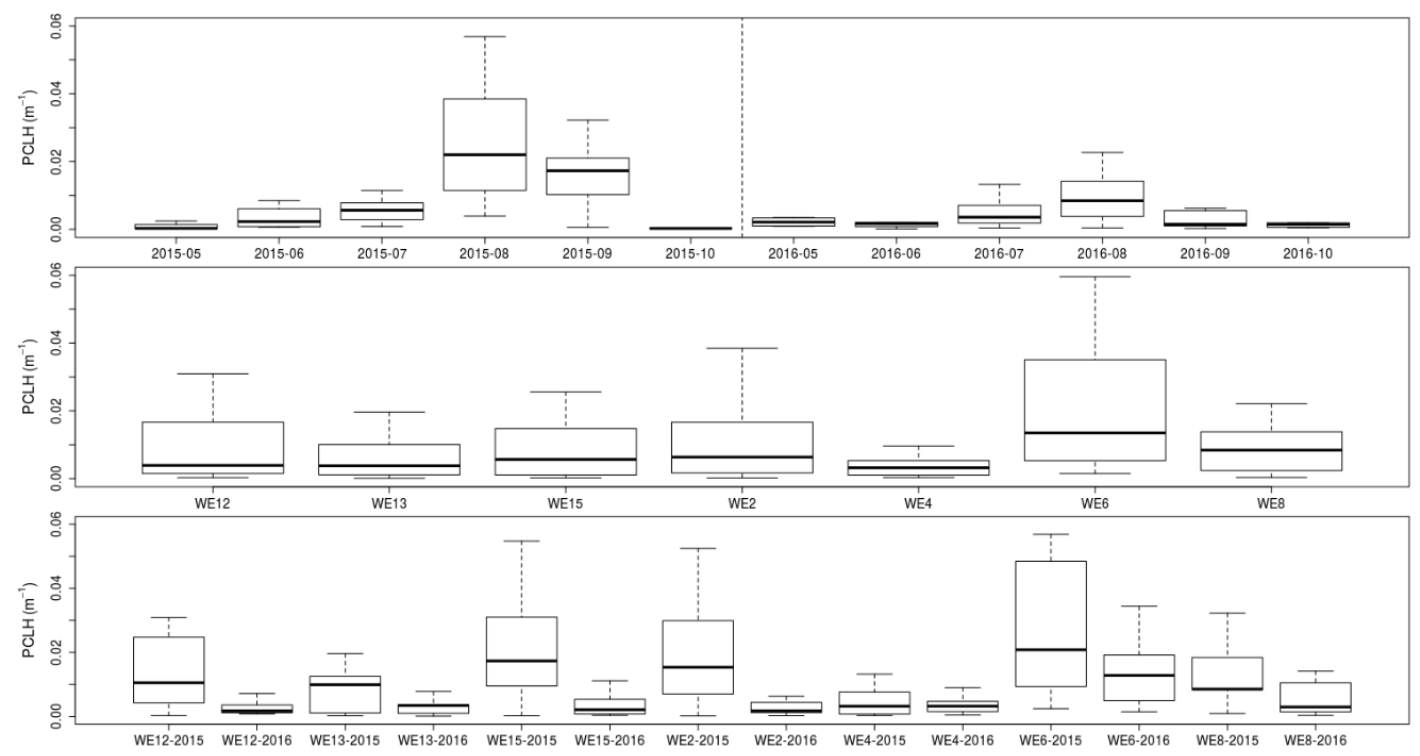

Figure 6.7. Spatial and temporal patterns of the particulate absorption phycocyanin line height (PCLH) in western Lake Erie expressed as boxplots where the black horizontal line is the median, the bottom and top of the box are the first and third quartiles respectively, and the horizontal gray lines below and above the box are the minimum and maximum values respectively. Monthly median values for 2015 (left half) and 2016 (right half) are shown in the top panel. Station median values for the combined 2015-2016 dataset are shown in the middle panel. Annual station median values are shown in the bottom panel.

The CDOM absorption spectral slope, $\mathrm{S}_{\mathrm{CDOM}}$, was analyzed to characterize the spatial and temporal variability of CDOM composition in western Lake Erie. The mean, median, and range of $\mathrm{S}_{\mathrm{CDOM}}$ values are also displayed in table 1. While $\mathrm{S}_{\mathrm{CDOM}}$ values exhibited similar range and median values overall for 2015 and 2016, significant temporal differences in

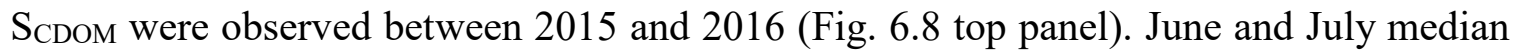

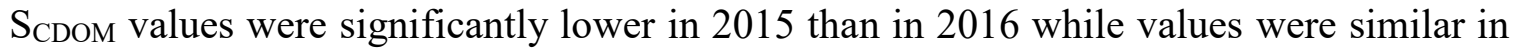
May, August, September, and October (June $p=0.015$, July $p=0.001$ ). Intra-monthly

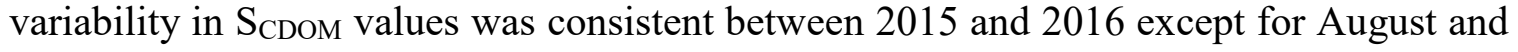
September where variability was larger in 2016 than in 2015. 
While significant temporal differences in $\mathrm{S}_{\mathrm{CDOM}}$ were documented, there were no significant differences in combined 2015-2016 median values for each station (Fig. 6.8 middle panel). Variance in station median $\mathrm{S}_{\mathrm{CDOM}}$ values were also similar with WE4 showing a slightly higher degree of variability than the other stations. Because of the increased variance in $\mathrm{S}_{\text {СDOM }}$ at WE4, its median value was significantly smaller than the overall combined study median value $\left(0.017 \mathrm{~nm}^{-1}, \mathrm{p}=0.036\right)$. There was also no significant difference between median $\mathrm{S}_{\mathrm{CDOM}}$ values between years for each station, although the minimum and maximum values were quite different for some stations between 2015 and 2016 (e.g. WE15) while others were not (e.g. WE2) (Fig. 6.8 bottom panel).
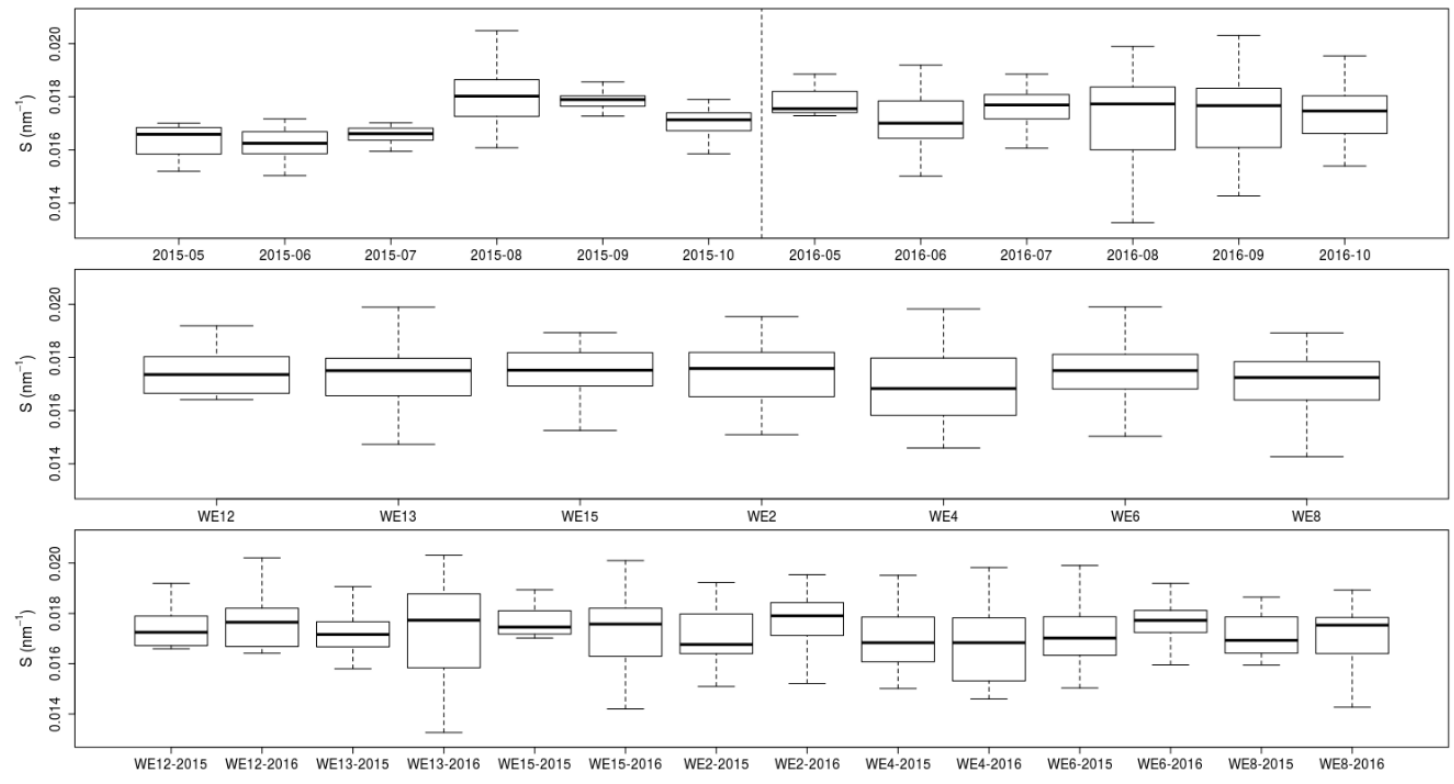

Figure 6.8. Spatial and temporal patterns of the CDOM spectral slope, $\mathrm{S}_{\mathrm{CDOM}}$, in western Lake Erie expressed as boxplots where the black horizontal line is the median, the bottom and top of the box are the first and third quartiles respectively, and the horizontal gray lines below and above the box are the minimum and maximum values respectively. Monthly median values for 2015 (left half) and 2016 (right half) are shown in the top panel. Station median values for the combined 2015-2016 dataset are shown in the middle panel. Annual station median values are shown in the bottom panel. 
In order to understand the spatial and temporal variability in scattering by particles (suspended solids and phytoplankton), the particulate beam attenuation $\left(\mathrm{c}_{\mathrm{p}}\right)$ spectral slope $(\gamma)$ was analyzed (Fig. 6.9 top panel). The mean, median, and range of $\gamma$ values are also displayed in table 1. Differences between months in 2015 and 2016 were evident, most notable where July 2015 was significantly greater than July 2016 ( $\mathrm{p}=0.01$ ), while 2016 September $(\mathrm{p}<0.001)$ and 2016 October $(\mathrm{p}=0.002)$ were larger than both months in 2015. Very similar values were observed in both August 2015 and 2016 corresponding to the dominant cyanoHAB occurrence period. Intra-monthly variance in $\gamma$ is greatest in May and October for both years corresponding to elevated river discharge and resuspension events. The single largest monthly variance occurred in July 2015 during the cyanoHAB initiation period where there were several large precipitation events resulting in very high discharge rates from the Maumee River (Michalak et al. 2013).

Spatial differences in $\gamma$ were observed between stations for the combined 2015-2016 dataset (Fig. 6.9 middle panel) most notably at WE4 which had median $\gamma$ values significantly lower than the other stations $(\mathrm{p}<0.001)$. Stations WE13 and WE4 exhibited the lowest $\gamma$ values and are located the furthest distance offshore relative to the Maumee River discharge plume. The highest values of $\gamma$ were observed at WE8, which also exhibited the greatest variability. Comparison of median $\gamma$ values for each station between years suggested general similarity with the exception of WE4 which had significantly lower values in 2015 than in 2016 ( $\mathrm{p}=0.007)$. While stations generally exhibited similar median values between years, there were differences in the range of $\gamma$ values observed at each station. For example, maximum $\gamma$ values at WE6 were approximately $60 \%$ greater in 2015 than in 2016 while maximum values were almost 50\% greater in 2016 than 2015 for WE13 (Fig. 6.9 bottom panel). 

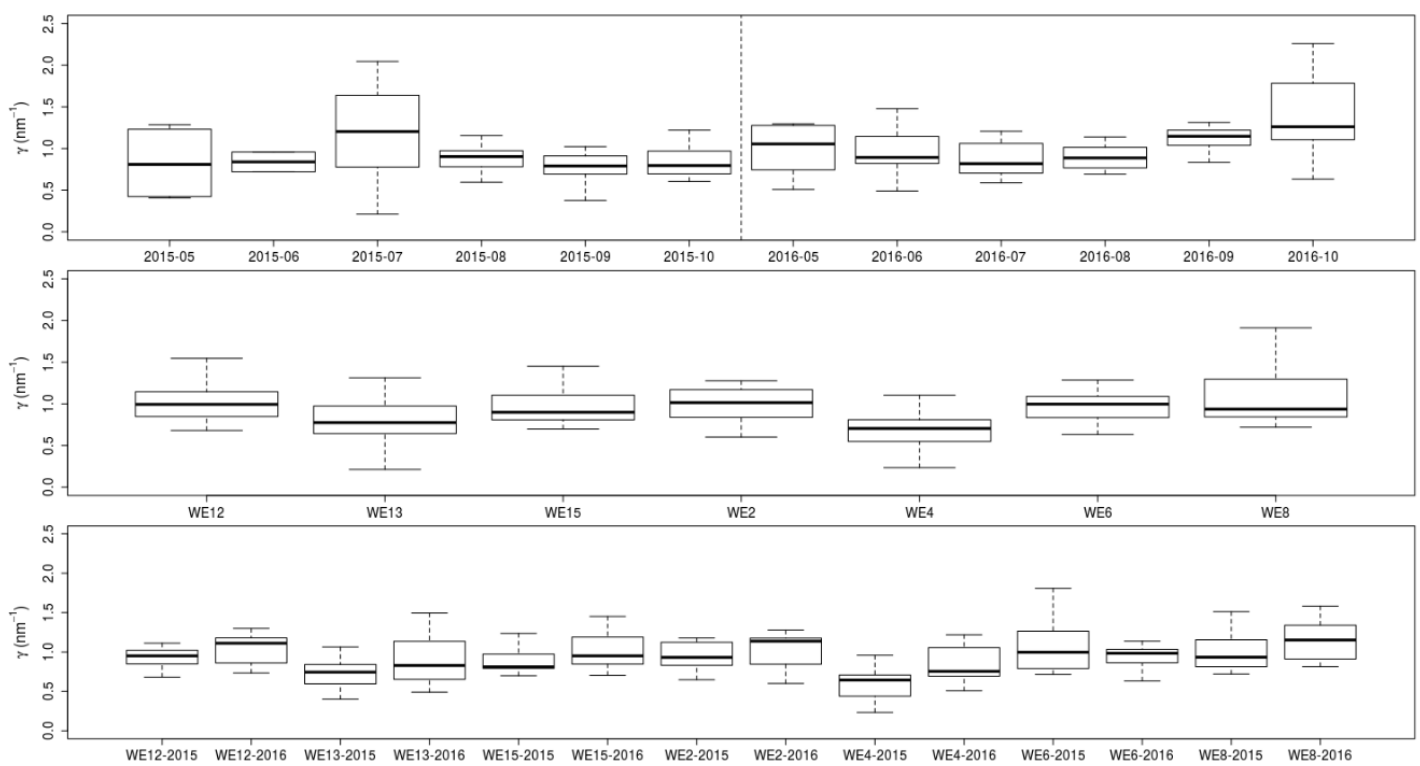

Figure 6.9. Spatial and temporal patterns of the particulate beam attenuation spectral slope, $\gamma$, in western Lake Erie expressed as boxplots where the black horizontal line is the median, the bottom and top of the box are the first and third quartiles respectively, and the horizontal gray lines below and above the box are the minimum and maximum values respectively. Monthly median values for 2015 (left half) and 2016 (right half) are shown in the top panel. Station median values for the combined 2015-2016 dataset are shown in the middle panel. Annual station median values are shown in the bottom panel.

In light of the large observed variability in western Lake Erie IOPs and optical proxies, it is expected that similar differences in measured remote sensing reflectance $\left(\mathrm{R}_{\mathrm{rs}}\right)$ would be observed. Mean $\mathrm{R}_{\mathrm{rs}}$ spectra were calculated for each month (using data from all stations) in 2015 and 2016 respectively and are shown in fig 6.10 (red lines). Variability in monthly spectra are also shown in the gray-black regions around the mean spectra where each color represents a percentage of measured $R_{r s}$ values, following the method described by Hochberg et al. (2003) (Light gray $=2.5-97.5 \%$, medium gray $=12.5-87.5 \%$, dark gray $=25-75 \%$ and black $=37.5-62.5 \%$ ). Note, no $\mathrm{R}_{\mathrm{rs}}$ data were recorded in May and October for 2016 due to the instrument undergoing maintenance. Within-year variability in mean spectral shape is evident in both 2015 and 2016. Early season (i.e. May-June) mean spectra 
for both years exhibit shapes typically associated with diatom presence (i.e. a wide and rounded peak between 500-600 nm), while mean spectra with cyanobacteria spectral features (e.g. troughs around $620 \mathrm{~nm}$ (PC absorption) and $676 \mathrm{~nm}$ (Chl-a absorption); peak around $705 \mathrm{~nm}$ (particle scattering)) occur in August for both years. A wide range in spectra magnitude is observed in June and October 2015, which is due to the presence of large sediment plumes characteristic of the elevated reflectance values in the red spectral region (i.e. $600-700 \mathrm{~nm}$ ). 

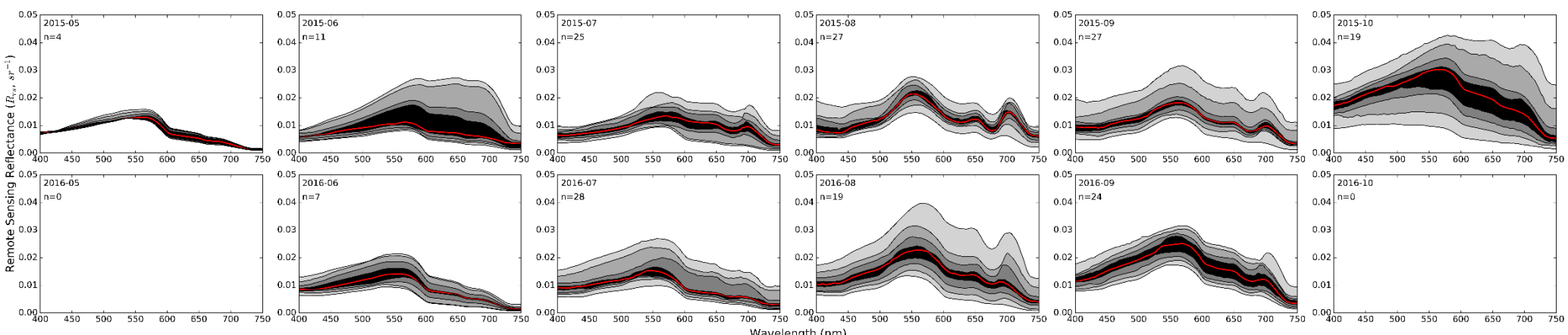

Figure 6.10. Temporal patterns in remote sensing reflectance $\left(\mathrm{R}_{\mathrm{rs}}, \mathrm{sr}^{-1}\right)$ in western Lake Erie for 2015 (top panels) and 2016 (bottom panels). The monthly mean spectra are displayed as red lines. Variability in monthly spectra are also shown in the gray-black regions around the mean spectra where each color represents a percentage of measured $\mathrm{R}_{\mathrm{rs}}$ values, following the method described by Hochberg et al. (2003) (Light gray $=2.5-97.5 \%$, medium gray $=12.5-87.5 \%$, dark gray $=25-75 \%$ and black $=37.5-62.5 \%$ ). Note, there were no $\mathrm{R}_{\mathrm{rs}}$ observations for May and October 2016. 
In addition to the monthly variability in $\mathrm{R}_{\mathrm{rs}}$, spatial variability was also evident. Fig 6.11 is an example of the variability in $\mathrm{R}_{\mathrm{rs}}$ between stations WE4 (top panels) and WE6 (bottom panels). Absolute $\mathrm{R}_{\mathrm{rs}}$ values are shown in the left panels while normalized $\mathrm{R}_{\mathrm{rs}}$ spectra are shown in the right panels. Reflectance spectra were normalized $\left(\mathrm{nR}_{\mathrm{rs}}(\lambda)\right)$ by dividing each spectra by its root sum of squares (Wei et al., 2016). A difference in the range in $\mathrm{R}_{\mathrm{rs}}$ magnitude between WE4 and WE6 is apparent, with WE6 exhibiting much higher $\mathrm{R}_{\mathrm{rs}}$ in the $550-700 \mathrm{~nm}$ spectral region likely the result of substantial sediment plumes. The normalized spectra (right panels) show a clear difference in spectral shapes between the stations with WE6 exhibiting a mean spectra with features characteristic of cyanobacteria while WE4 is more typical of other phytoplankton assemblages. Both stations display spectra associated with surface scums events (peak $\sim 705 \mathrm{~nm}$ ) however; these scums appear to be more frequent at WE6.

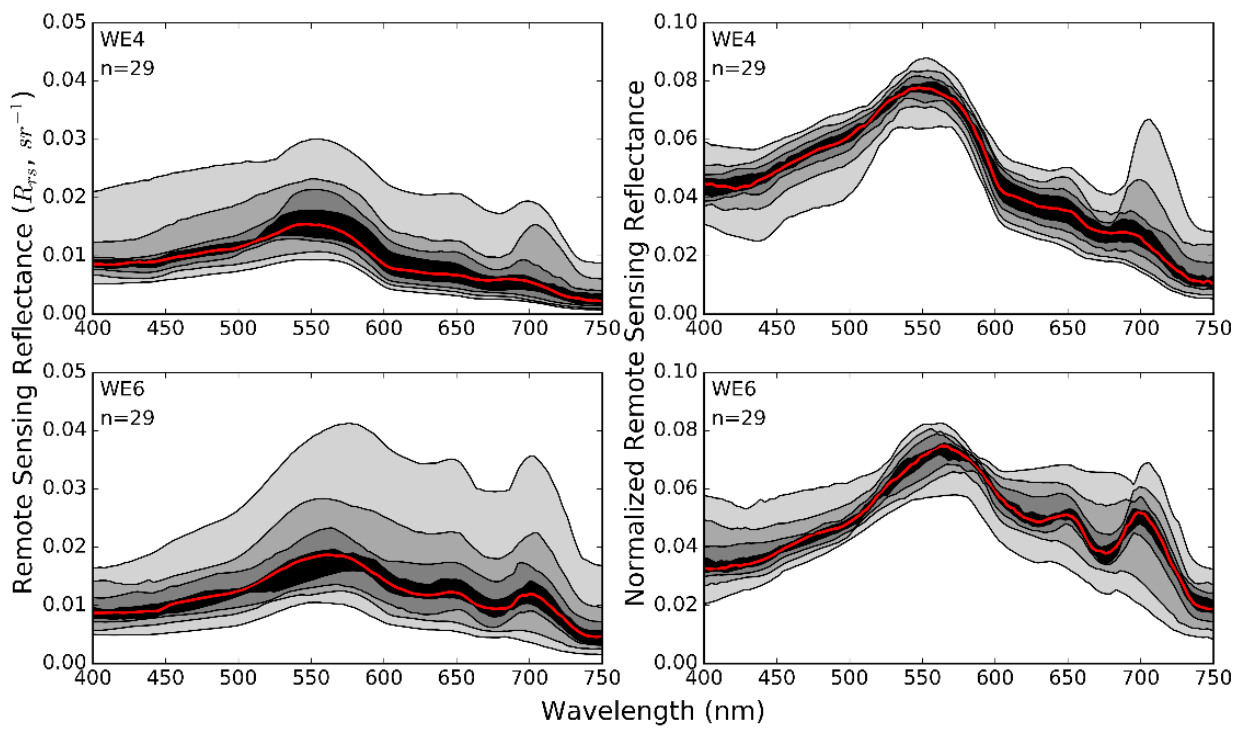

Figure 6.11. Study period mean remote sensing reflectance $\left(\mathrm{R}_{\mathrm{rs}}, \mathrm{sr}^{-1}\right)$ for stations WE4 (top panels) and WE6 (bottom panels). Absolute $\mathrm{R}_{\mathrm{rs}}$ (left panels) and normalized $\mathrm{R}_{\mathrm{rs}}$ (right panels) are shown for both stations. The study period mean spectra are displayed as red lines. Variability in study period spectra are 
also shown in the gray-black regions around the mean spectra where each color represents a percentage of measured $\mathrm{R}_{\mathrm{rs}}$ values, following the method described by Hochberg et al. (2003) (Light gray $=2.5-97.5 \%$, medium gray $=12.5-$ $87.5 \%$, dark gray $=25-75 \%$ and black $=37.5-62.5 \%$ ).

Because of the similar variability observed in both IOPs and AOPs $\left(\mathrm{R}_{\mathrm{rs}}\right)$ and the fact that $\mathrm{R}_{\mathrm{rs}}$ shape and magnitude are a function of IOPs it is expected that the IOP proxy variability should correlate with changes in $\mathrm{R}_{\mathrm{rs}}$. An example of this relationship is shown in figure 6.12 which is a scatterplot between $\mathrm{PCLH}_{\mathrm{ap}}$ (y-axis) and $\mathrm{PCLH}_{\mathrm{Rrs}}$ (x-axis). $\mathrm{PCLH}_{\mathrm{Rrs}}$ was calculated from the measured $\mathrm{R}_{\mathrm{rs}}$ spectra using the same line height approach and the same wavelengths as the $\mathrm{PCLH}_{\mathrm{ap}}$ (refer to methods section). Because the spectral feature of interest (PC absorption) is a trough in the $\mathrm{R}_{\mathrm{rs}}$ spectra the $\mathrm{PCLH}_{\mathrm{Rrs}}$ values are negative as opposed to positive for the $\mathrm{PCLH}_{\mathrm{ap}}$. In figure 6.12, the $\mathrm{PCLH}_{\mathrm{Rrs}}$ values were multiplied by -1 in order to express the values as positive numbers. Samples shown on figure 6.12 are binned into two groups where the lab based $\mathrm{PC} / \mathrm{Chl}$-a ratio was less than or equal to 0.1 (blue dots) and greater than 0.1 (red dots). These groups represent samples dominated by PC (red dots) and Chl-a (blue dots) respectively. The plot reveals a positive trend between $\mathrm{PCLH}_{\mathrm{ap}}$ and PCLH $\mathrm{Prs}_{\text {rs }}$ for PCLH $\mathrm{Rrs}_{\text {rs }}$ values greater than approximately $0.00075 \mathrm{sr}^{-1}$. Note that the majority of Chl-a dominated samples (blue dots) result in a very small change in

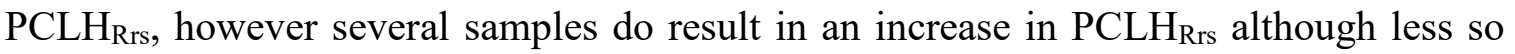
than those PC dominated samples. Many of the PC dominated samples (red dots) positively correlate with $\mathrm{PCLH}_{\mathrm{Rrs}}$ however with a high degree of variability. This variability is expected as $\mathrm{R}_{\mathrm{rs}}$ is controlled by both absorption as well as backscatter, the latter of which is not accounted for in this plot. Never the less, figure 6.12 suggests observed changes in $R_{\mathrm{rs}}$ at $620 \mathrm{~nm}$ are at least partially explained by changes in particulate absorption, which is highly correlated with increased PC abundance (recall figure 6.6). This result is the basis for remote sensing algorithms aiming to retrieve PC concentration (e.g. Simis et al. 2005). 


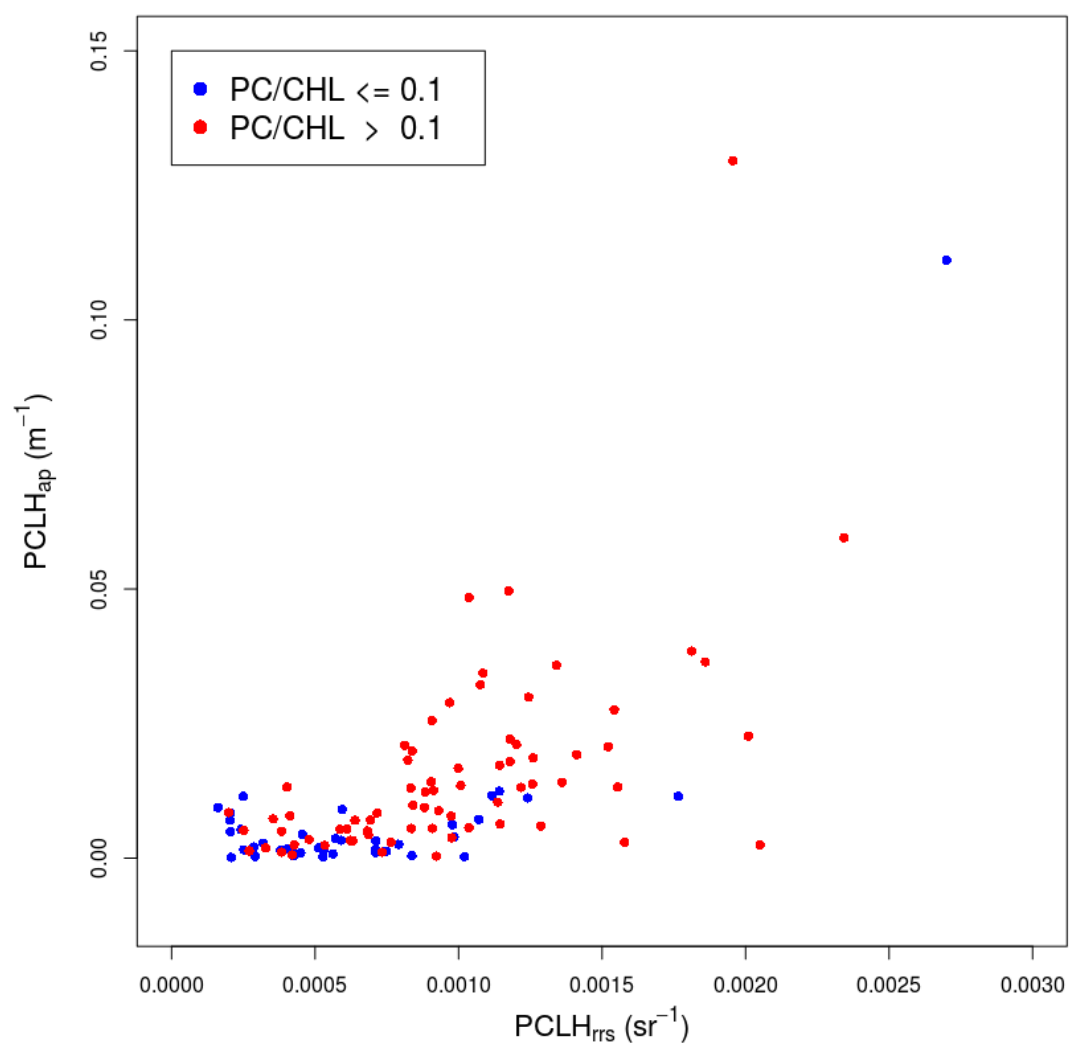

Figure 6.12. Comparison between particulate absorption phycocyanin line height $\left(\mathrm{PCLH}_{\mathrm{ap}}\right)(\mathrm{y}$-axis $)$ and remote sensing reflectance phycocyanin line height $\left(\mathrm{PCLH} \mathrm{Rrs}_{\mathrm{s}}\right)$ (x-axis). Observations where the phycocyanin to chlorophylla ratio $(\mathrm{PC} / \mathrm{CHL})$ is low $(<=0.1)$ are shown as blue dots while high $\mathrm{PC} / \mathrm{CHL}$ ratio $(>0.1)$ observations are displayed as red dots.

\subsection{Discussion}

A unique aspect of this study is the documentation of significant temporal IOP variability over the May-October period for two full years (2015-2016). Overall, very limited temporal IOP observations exist in the Great Lakes as a whole and are mostly limited to two samplings from different years (Perkins et al. 2013). Several studies characterizing IOPs in Lake Erie have been limited to cruises spanning several days with station revisits on monthly time scales. Binding et al. (2008) conducted IOP sampling cruises in September 2004 and May, July, and September 2005 with the sampling stations located throughout 
the western, central, and eastern basins of Lake Erie. O'Donnell et al. (2010) made IOP observations in the western basin of Lake Erie in September 2007 but they only occurred over a two-day period. Because of the limited temporal sampling of these studies, it is likely that only a subset of the range in IOP and optically active constituent variability was quantified. For example, Binding et al. (2008) reported the range of асдом at $440 \mathrm{~nm}$ as 0.08 to $0.75 \mathrm{~m}^{-1}$ while for this study the range was 0.05 to $6.74 \mathrm{~m}^{-1}, \mathrm{a} \sim 9$-fold difference in maximum value, with the highest values occurring in July and August. Similarly, O'Donnell et al. (2010) noted a range in the particulate scattering $\left(b_{p}\right)$ spectral slope of 0.28 to -1.0 while for this study the range was from 0.57 to -2.81 indicating vastly different particle size distributions were encountered in each study. Only recently reported are IOP observations (Moore et al. 2017) from the month of August, which represents the dominant cyanoHAB period and many times encapsulates the annual peak bloom extent (Wynne et al. 2015, Sayers et al. 2019 in this issue). While Moore et al. 2017 provided a very useful bio-optical characterization of cyanobacteria blooms in WBLE, the study periods were limited to the August period for two years, which limits the ability to evaluate how IOPs vary during bloom initiation and senescence. Moreover, little is known about the variability in IOPs in WBLE between years on short time scales. The near weekly sampling for two full years presented in this study provides the necessary temporal scale to better understand WBLE optical dynamics.

A very interesting component of this study is the documentation of the extreme variability in mean monthly IOPs for 2015 and 2016 in the WBLE. For example, the magnitudes in beam attenuation (c), absorption (a), and scattering (b) for October 2015 (high) and 2016 (low) span the entire range of values for all other months and years combined. It is not unexpected that large variability between October 2015 and 2016 was observed, as this is a period of rapid transition in environmental conditions in Lake Erie. Water temperatures are dropping quickly with increasing precipitation and cloud cover, and sediments are being re-suspended from increased wave action. Moreover, as observed in 2011, very large but short-lived (less than 7 days) cyanoHAB events can also occur but these blooms do not occur every year (Michalak et al. 2013). The large IOP variability observed in October 
indicates the necessity of regular in situ sampling during the algal bloom periods in Lake Erie. This variability also suggests that tight satellite and in situ matchup restrictions (e.g. minimal difference in time between measurement acquisition and satellite overpass) should be considered in order to ensure valid comparisons are made. It is also generally evident from Figure 3 that IOP values were higher in 2015 than in 2016, which corresponds with the significantly larger cyanoHAB occurrence in 2015 (Sayers et al. 2018 in this issue). Annual cyanoHAB events in Lake Erie have been shown to be highly variable from yearto-year partially due to varying spring discharge rates from the Maumee River (Stumpf et al. 2016, Sayers et al. 2016). Sayers et al. also presented a relationship between cyanoHAB extents and the number of sediment re-suspension events that are driven by the regional meteorology (wind speed) and basin morphology (depth). Using these predictors (spring discharge and re-suspension events), 2015 is expected to have experienced larger more sustained cyanoHABs than 2016, which is consistent with the IOP observations presented in Figure 6.3.

Maumee River discharge rates are also contributing to temporal variability of IOPs. This is evidenced by the almost 25 -fold difference in mean monthly discharge between July $2015\left(19,300 \mathrm{ft}^{3} / \mathrm{s}\right)$ and July $2016\left(794 \mathrm{ft}^{3} / \mathrm{s}\right)$ which corresponds to an approximate 1.5 -fold difference in $b_{p}(440)$, greater than 2-fold difference in $a_{p g}(440 \mathrm{~nm})$, and 1.6-fold difference in $c_{p g}(400 \mathrm{~nm})$. Because July is often the first month of bloom initiation in WBLE the large variability is not surprising as bloom dynamics often change between years. On the other hand, much smaller differences in IOP magnitude were observed between August 2015 and 2016, yet the IOP values were generally near the highest for all months and years combined. Because August is usually within the mid-point of the annual bloom cycle these results are not surprising and indicate a relatively stable period between years, with little variability due to river discharge (August $2015=1567 \mathrm{ft}^{3} / \mathrm{s}$, August $2016=640 \mathrm{ft}^{3} / \mathrm{s}$ ) but high IOP values are likely due to persistent cyanoHAB presence. Aggregation of IOPs by month allows for simple comparison and identification of seasonal patterns between years and can help link observations to significant controlling factors. Because these seasonal patterns can change between years due to difference in bloom dynamics and other factors 
it is critical to monitor IOPs on at least a monthly scale for the June through October period for every year of interest.

One of the most interesting and unique results from this study is the documentation of seasonal variability in $\mathrm{a}_{\mathrm{ph}} * 665$ over the course of the vegetative season for two years. The range in $\mathrm{aph}_{\mathrm{ph}} * 665$ observed in this study was similar to the range reported by Moore et al. (2017) (their Figure 5b) but less than documented by Binding et al. (2008) which observed an order of magnitude larger maximum value. Mean $\mathrm{a}_{\mathrm{ph}}{ }^{*} 665$ values for 2015 (0.0034 $\left.\mathrm{m}^{2} \mathrm{mg}^{-1}\right)$ and $2016\left(0.0038 \mathrm{~m}^{2} \mathrm{mg}^{-1}\right)$ were 2-fold smaller than the mean value $\left(0.007 \mathrm{~m}^{2} \mathrm{mg}^{-}\right.$ $\left.{ }^{1}\right)$ reported by Binding et al. (2008) and appear to be lower than those depicted (their Figure $5 b$ ) in Moore et al. (2017) although no mean values were reported for the $665 \mathrm{~nm}$ wavelength. It is important to note that in this study $\mathrm{aph}_{\mathrm{ph}} * 665$ was calculated from in situ ac-s measurements of $a_{p}$ using a line height approach, while the other studies were derived spectrophotometrically using filtered water samples that had been measured for both phytoplankton absorption and non-algal particle absorption. Because of the differences in these two approaches, it is expected that the $\mathrm{a}_{\mathrm{ph}}{ }^{*} 665$ calculated in this study would be smaller than those calculated using lab spectrophotometry. Roesler and Barnard (2013) noted a factor of two lower value using the line height method for samples from the marine environment. Assuming a factor of two difference between the two methods, our study mean $\mathrm{a}_{\mathrm{ph}} * 665$ value would be very close to that reported by Binding et al. (2008). Further investigation into coherence of these two methods is needed to understand how intercomparable their values are moving forward.

A steady decline in median $\mathrm{a}_{\mathrm{ph}} * 665$ values from May through August is evident in both years indicating a steady shift in the phytoplankton community size structure from smaller to larger size populations. The lowering of $\mathrm{aph}_{\mathrm{ph}} * 665$ is attributable to the pigment package effect (Bricaud et al. 1995) which is a function of pigment abundance and how pigments are arranged within the cell. Larger cells tend to exhibit higher degrees of pigment packaging thus lower $\mathrm{aph}_{\mathrm{ph}}$ * values in general. As pointed out in Moore et al. (2017) some of the observed packaging effect (i.e. lower $\mathrm{a}_{\mathrm{ph}}{ }^{*}$ values) may be due in part to the formation 
of large cyanobacteria colonies which could impart a degree of "packaging" well beyond that observed for individual cyanobacteria cells (Matthews and Bernhard 2013, Zhang et al. 2012). Interestingly, median $\mathrm{a}_{\mathrm{ph}} * 665$ in August 2015 were significantly less than those for 2016, likely indicating less abundance of large cyanobacteria colonies in 2016. Similarly, the lowest median $\mathrm{a}_{\mathrm{ph}} * 665$ values for 2016 were observed in October suggesting the increased presence of larger sized phytoplankton or formations of large cyanobacteria colonies. The difference in the annual trend of $\mathrm{aph}^{*} 665$ between 2015 and 2016 is a very interesting observation which suggests a very different algal community/size progression for each year. Other studies reporting values of $\mathrm{a}_{\mathrm{ph}}{ }^{*} 665$ (Binding et al. 2008, Moore et al. 2017) do not have the fine scale temporal granularity over the full vegetative season to document these observed transitions, which is critical information for remote sensing biooptical models that utilize values of $\mathrm{a}_{\mathrm{ph}} * 665$ for initial parameterization (Shuchman et al. 2013).

In light of the observed temporal trends in $\mathrm{a}_{\mathrm{ph}} * 665$ observations, it was somewhat surprising there were no significant differences in $\mathrm{a}_{\mathrm{ph}} * 665$ between stations over the combined two-year study period. Studies that also report $\mathrm{a}_{\mathrm{ph}}{ }^{*}$ values for Lake Erie did not document variability at a particular station over long time periods but rather at the basin scale, further confirming the novelty of this dataset. Because $a_{\mathrm{ph}}{ }^{*} 665$ values were consistent for stations over the combined two-year period, it would suggest that the fluctuations in phytoplankton/cyanobacteria assemblages over the season occur uniformly across the area represented by the sampling stations. A possible exception may be for station WE4 where the greatest variability in $\mathrm{a}_{\mathrm{ph}} * 665$ was observed. WE4 is located approximately equidistant from the mouths of the Maumee and Detroit Rivers and is situated in $8.5 \mathrm{~m}$ deep water. This location appears to be in a transition zone between predominantly Maumee River water and Detroit River water that can switch between the two depending on currents and flows. Moore et al. (2017) documented this transition in optical properties from the Detroit Plume to Maumee Bay water and noted that this frontal boundary is always present but varies in location depending on "climatic factors". This observation is consistent with the increased variability in IOPs at WE4 relative to other 
stations not in this transition area. Because $\mathrm{a}_{\mathrm{ph}} * 665$ tends to vary uniformly for stations over the season (exception for WE4), it may be possible to assume a temporal mean $\mathrm{aph}_{\mathrm{ph}}$ *65 value for remote sensing model parameterization for waters inclusive of the sampling stations in this study.

This study is the first to document the use of the particulate absorption line height method, using ac-s data, at the PC absorption peak $(\sim 620 \mathrm{~nm})$ to track cyanobacteria abundance. Not only was there a strong correlation between individual sample $\mathrm{PCLH}_{\mathrm{ap}}$ and $\mathrm{PC}$ concentration (figure 11, Pearson's correlation 0.72), the monthly median $\mathrm{PCLH}_{\mathrm{ap}}$ values for 2015 and 2016 clearly follow the same patterns identified in the median laboratory extracted PC concentrations (Figure 2, Pearson's correlation $=0.96$ ) indicating the method is sensitive to varying levels of pigment abundance. The temporal trends in $\mathrm{PCLH}_{\text {ap }}$ suggest a much more intense cyanoHAB occurrence in 2015 relative to 2016, with both peaking in August. In terms of spatial extent, the cyanoHAB bloom was more intense in 2015 for all stations except WE4, which showed very similar median $\mathrm{PCLH}_{\text {ap }}$ values between years. The lack of a large median PCLH ap value at WE4 in 2015 suggests the bloom was only periodically occurring at that location and not of particularly high abundance, which agrees with the notion of WE4 being located in a dynamic transition zone between Detroit River and Maumee River waters. These observations are consistent with those reported by Sayers et al. 2019 (in this issue) that mapped the areal extents of cyanoHAB blooms for 2015 and 2016 using a modified Chl-a retrieval algorithm. It should be acknowledged that the $a_{p}$ around $620 \mathrm{~nm}$ above the estimated aNAP baseline may not be exclusively due to PC. Chla exhibits a small absorption peak in the $620 \mathrm{~nm}$ range that may also be included in the $\mathrm{PCLH}_{\mathrm{ap}}$ value which is a possible source of variability when comparing $\mathrm{PCLH}_{\mathrm{ap}}$ to laboratory derived PC concentrations. The good agreement between $\mathrm{PCLH}_{\mathrm{ap}}$ and laboratory PC concentrations confirms the utility of using this spectral feature in remote sensing algorithms to estimate PC (Simis et al. 2005) that have been developed for sensors with wavelengths in this region (algorithm review in Mishra et al. 2017). 
The CDOM absorption spectral slope, $\mathrm{S}$, values reported in this study were significantly variable over the two-year study period with minimum and maximum values exceeding those previously reported. The CDOM spectral slope has been shown to relate to the molecular weight of the acids (humic and fulvic) comprising the dissolved material. The ratio of humic acids to fulvic acids have been used as a proxy for CDOM composition (e.g. from phytoplankton decomposition or decay of terrestrial material) (Carder et al. 1989; Yacobi et al. 2003). Regardless of the CDOM composition, the spectral slope is a critical component to incorporate in multi-spectral remote sensing bio-optical models

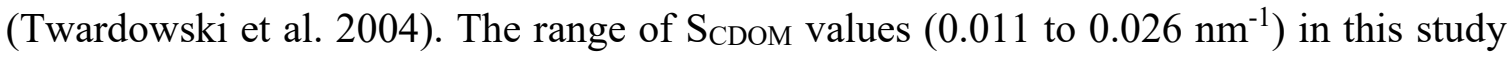
was twice as large as those reported in Binding et al. (2008) $\left(0.0128\right.$ to $\left.0.0197 \mathrm{~nm}^{-1}\right)$ and five-fold greater than observed by Moore et al. (2017). This range is almost identical to that reported by Babin et al. (2003) (0.011 to $0.025 \mathrm{~nm}^{-1}$ ) for a wide range of coastal

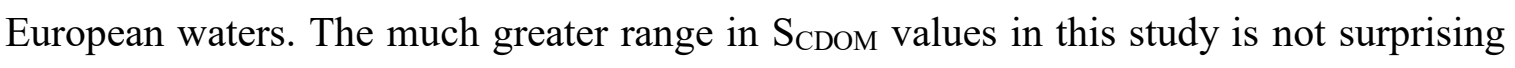
given that the maximum value of асдом 443 was $\sim 9$ fold greater than reported in Binding et al. (2008) and almost 6 fold more than what Moore et al. (2017) observed. Given the wide ranges of both $\mathrm{S}_{\text {CDOM }}$ and асDOM $_{4} 43$ between the three studies, the mean $\mathrm{S}_{\text {CDOM values }}$ of each were in relative agreement with Binding et al. (2007) and Moore et al. (2017) reporting 0.0161 and $0.018 \mathrm{~nm}^{-1}$, respectively, while the overall mean for this study was $0.0173 \mathrm{~nm}^{-1}$. Much of the range in $\mathrm{S}_{\mathrm{CDOM}}$ values was a result of much larger monthly fluctuations in 2016 especially in August and September with much less variability observed in 2015. This would suggest much greater variations in allochthonous and autochtonous sources of CDOM in 2016 relative to 2015, possibly due to differences in river discharge fluctuations or cyanoHAB abundance between years. Binding et al. (2008) suggested that the preferential adsorption of higher molecular weight CDOM on to suspended mineral particles, leaving only the lower weight fraction free in the water, may alter the bulk absorption properties one may expect to observe, which may well be occurring in these measurements also altering observed patterns.

This study also documents the first reported values of the particulate beam attenuation $\left(\mathrm{c}_{\mathrm{p}}\right)$ spectral slope, $\gamma$, in the Great Lakes. To first order the $c_{p}$ spectral slope has been shown to 
relate to the power law slope of the particle size distribution (PSD) both theoretically (Boss et al. 2001) and experimentally (Slade and Boss 2015), potentially providing information into phytoplankton community structure and sediment transport. The observed range of values of $\gamma$ in this study was more than two-fold larger than observed in coastal ocean waters (Slade and Boss et al. 2015) and the bottom boundary layer in the mid-Atlantic Bight (Boss et al. 2001). The large variability in $\gamma$ suggests highly variable PSDs throughout the two-year period in western Lake Erie with largest fluctuations occurring in July 2015 corresponding to extremely high mean discharge (19300 ft $\mathrm{ft}^{3} / \mathrm{s}$ compared with long term July mean discharge $2850 \mathrm{ft}^{3} / \mathrm{s}$ ) from the Maumee River. August and September for both years exhibited the most consistent $\gamma$ values suggesting very similar PSDs during the dominant cyanoHAB period while experiencing relatively low discharge rates $(<1600$ $\mathrm{ft}^{3} / \mathrm{s}$ ). There was little spatial difference in $\gamma$ values with only WE4 exhibiting significantly lower values than all other stations. The lower $\gamma$ values would suggest the presence of a PSD with relatively larger particles likely in the form of large diatoms or other microplankton and less occurrence of small particles consistent with inorganic sediment particles either re-suspended or injected from the river. It should be noted that the acceptance angle ( 0.93 degrees) on the beam attenuation chamber optic of the ac-s can act as a particle size "filter" for particles larger than $50 \mu \mathrm{m}$ (Boss et al. 2009, Slade and Boss et al. 2015). Additionally, the relationship of $\gamma$ to the PSD slope assumes the PSD slope follows the form of a negative power law as a function of particle diameter (Bader 1970), which may not be true in many environments including complex freshwater systems. Boss et al. (2001) also note the presence of strongly absorbing particles may limit the robustness of the relationships between $\gamma$ and PSD due to anomalous dispersion. Further research into the relationship between $\gamma$ and PSDs in the Great Lakes, and specifically Lake Erie, is needed to understand these dynamics.

In light of the observed spatial and temporal variability in IOPs and optical proxies in western Lake Erie, uncertainties in remote sensing algorithm retrievals that use fixed model parameterizations need to be evaluated spatially and temporally. Bio-optical remote sensing algorithms rely on knowing some information about the underlying IOPs of the 
water being sensed (Werdell et al. 2018). Previous investigators have formulated retrieval algorithm frameworks that make assumptions regarding the underlying IOPs, usually from a set of in situ measurements or from reported literature. For example, Binding et al. (2012) proposed an approach to simultaneously retrieve concentrations of minerogenic suspended particulate matter (MSPM) and Chl-a using the red and near-infrared bands of the MODIS Aqua sensor. The Binding et al. (2012) algorithm relates the satellite-observed water leaving radiance at 667 and $748 \mathrm{~nm}$ to the water volume absorption and backscatter coefficients using mean mass specific absorption and backscattering coefficients for phytoplankton and non-algal particles $\left(\mathrm{a}_{\mathrm{ph}}{ }^{*}\right.$ and $\left.\mathrm{a}_{\mathrm{NAP}}{ }^{*}\right)$. Through these assumptions, concentrations of MSPM and Chl-a can be estimated. Similarly, Simis et al. (2005) proposed a bio-optical algorithm to retrieve PC concentration through the estimation of PC absorption $\left(\mathrm{a}_{\mathrm{pc}}\right)$ at $620 \mathrm{~nm}$. The algorithm related the ratio of reflectance at 709 and 620 $\mathrm{nm}$ to a model for PC absorption and then to PC concentration using an assumed $\mathrm{apc}_{\mathrm{pc}}$ * value. Finally, Shuchman et al. (2013) reported a bio-optical inversion model to retrieve concentrations of the primary optically active constituents (OACs) Chl-a, CDOM absorption, and fixed suspended solids (FSS) from multi-spectral MODIS imagery. The model relates concentrations of the OACs to observed reflectance values using assumed values for $\mathrm{aph}_{\mathrm{ph}} * \mathrm{~S}_{\mathrm{CDOM}}$, and $\mathrm{a}_{\mathrm{NAP}} *$ to minimize the difference between measured and modeled reflectance at all MODIS bands.

Though each of these model frameworks retrieve different water parameters, they all rely on parameterization of specific IOPs, which have been shown in this study to vary in time and space. Variable model parameterization may yield lower uncertainties in model retrievals when deployed throughout the ice-free period. For example, the $\mathrm{a}_{\mathrm{ph}} * 665$ value used in the Binding et al. (2012) model is $0.025 \mathrm{~m}^{2} \mathrm{mg}^{-1}$, which is approximately 1.6 times greater than the largest $\mathrm{aph}_{\mathrm{ph}} * 665$ value observed in this study $\left(0.015 \mathrm{~m}^{2} \mathrm{mg}^{-1}\right)$ and almost seven times greater than the study mean value $\left(0.004 \mathrm{~m}^{2} \mathrm{mg}^{-1}\right)$. Even after transforming the $\mathrm{a}_{\mathrm{ph}} * 665$ values in this study to values comparable in magnitude with Binding et al. (2012) (i.e. multiply by a factor of two, Roesler and Barnard (2013)), the transformed study mean value $\left(0.008 \mathrm{~m}^{2} \mathrm{mg}^{-1}\right)$ is still a factor of three lower than the $0.025 \mathrm{~m}^{2} \mathrm{mg}^{-1}$ value used in 
Binding et al. (2012). It is possible that an adaptive parameterization of $\mathrm{a}_{\mathrm{ph}} * 665$ in the Binding et al. (2012) model over the observed range of values could provide more accurate retrievals of both Chl-a and FSS in both cyanobacteria and non-cyanobacteria conditions. Additionally, Simis et al. (2005) acknowledge the high degree of variability of $a_{p c} * 620$ in response to changing environmental conditions and suggest that if specific knowledge of the variability in $a_{\mathrm{pc}} * 620$ is known or can be estimated for a particular environment of interest, more accurate retrievals should be attainable. Similar to the other two model frameworks, the Shuchman et al. (2013) approach assumes a $S_{C D O M}$ value of $0.016 \mathrm{~nm}^{-1}$ which is similar to that reported in Binding et al. (2008) $\left(0.0161 \mathrm{~nm}^{-1}\right)$ but lower than the mean values reported by Moore et al. (2017) $\left(0.018 \mathrm{~nm}^{-1}\right)$ and in this study $\left(0.0173 \mathrm{~nm}^{-1}\right)$. In addition, the large range of $\mathrm{S}_{\mathrm{CDOM}}$ observed in this study suggests that the model default parameterization may be significantly different from the actual value for a given date and location depending on environmental factors. Further investigation into how remote sensing algorithm accuracy and uncertainty varies as a function of different parameterizations is warranted and should ultimately lead to more accurate water quality products.

\subsection{Conclusion}

Presented in this study is a unique IOP data set for western Lake Erie that spans the vegetative season over two full years. Weekly observations at the same locations over a long period (six months) provides insights into the natural variability of IOPs as a function of changing algal communities, river discharge fluctuations, and various other environmental forcing functions. Spatial variations in IOPs were identified and related to the proximity of a station to significant river discharge plumes. Significant variability in IOPs shape and magnitude were observed on weekly and monthly scales suggesting in situ measurements should be collected at least monthly in western Lake Erie to adequately track the significant variability. 
This study documented significant shifts in the mass specific phytoplankton absorption coefficient, $\mathrm{a}_{\mathrm{ph}} * 665$, from the May through August period associated with the development of significant colonial cyanobacteria blooms. Differences in annual progression and magnitude of $\mathrm{a}_{\mathrm{ph}} * 665$ between 2015 and 2016 suggests the relative intensity of the annual bloom is a dominant factor controlling the distribution of $\mathrm{a}_{\mathrm{ph}} * 665$ values in western Lake Erie from year to year. Further measurements of $a_{p h} * 665$ at short time scales over the full vegetative season are needed to fully understand how shifts in algal communities vary as a function of cyanoHAB extent and intensity.

Other IOP variables displayed significant variability over the two-year dataset with the $\mathrm{PCLH}_{\mathrm{ap}}$ exhibiting a seasonal trend in association with the cyanoHAB progression, while $\mathrm{S}_{\mathrm{CDOM}}$ and the $\mathrm{c}_{\mathrm{p}}$ spectral slope exhibited variability more associated with environmental forcing functions. Fully understanding the complex IOP variability associated with unpredictable driving factors, such as increased discharge rates from high precipitation events and wind driven sediment re-suspension, will require further investigation and targeted sampling efforts to capture the full range of optical variability over these relatively short-lived events.

Finally, remote sensing water quality retrieval algorithms that assume a single IOP parameterization can yield meaningful results on the average (e.g. annually), however, significant errors may be present for time periods and locations where the model optical parameters are significantly different than the actual properties. The IOP dataset presented in this study has shed some light on how these properties change over the course of a season and between years, and may be useful in advancing the applicability and robustness of remote sensing methods to retrieve valuable water quality information in western Lake Erie.

\subsection{Acknowledgements}

This study was funded by the Cooperative Institute for Great Lakes Research (CIGLR) and the NOAA Great Lakes Environmental Research Lab (GLERL) under contract \#3004701270. Aspects 
of this study were also funded by the NASA Carbon Monitoring System (CMS) under contract \#80NSSC17K0712. We would like to thank NOAA GLERL and their ship captains for facilitating data collection on their research vessels. This is GLERL contribution number 1915 funded under the Great Lakes Restoration Initiative EPA/NOAA Interagency Agreement \# DW-013-92492801. This publication is contribution \#1139 from the Cooperative Institute for Great Lakes Research, University of Michigan under the NOAA Cooperative Agreement NA17OAR4320152. 


\section{Conclusion}

The general research hypothesis "electro-optical (EO) remote sensing can be used to provide robust characterization and unique insights of phytoplankton dynamics at community and population scales in a variety of freshwater systems" was supported through the work in this dissertation. In this dissertation work I developed an approach to quantify global summer phytoplankton community abundance using a newly developed remote sensing derived chlorophyll-a product. This product was also used in conjunction with a newly created carbon fixation model to assess global freshwater phytoplankton production which provided new insights into the role freshwater systems play in the global carbon budget. Spatial and temporal assessments of specific populations of phytoplankton and cyanobacteria were established through the development of a new remote sensing algorithm to isolate high biomass assemblages in the Laurentian Great Lakes (Lake Erie, Saginaw Bay, Green Bay). The algorithm was developed to facilitate the fusion of multiple remote sensing data sources (SeaWiFS and MODIS) in order to generate a new 20-year time-series data product to better understand the factors controlling bloom dynamics. Finally, a spatio-temporal analysis documenting the variability of inherent optical properties (IOPs) in Lake Erie established a seasonal progression of phytoplankton/cyanobacteria community structures for two years over the vegetative season, the findings of which are critical for the development of the next generation of hyperspectral remote sensing algorithms to improve phytoplankton community characterizations from space. These documented results clearly show the utility of EO remote sensing to provide characterization of phytoplankton dynamics and insights at both community and population scales in freshwater systems.

A very important aspect of this dissertation work is the development of several new remote sensing algorithms to assess phytoplankton dynamics at global and local scales. A simple depth integrated model (DIM) to estimate phytoplankton production was created for freshwater systems. This model is a refinement of the Light Utilization Index (LUI) proposed by Falkwoski (1981) for marine environments which requires only basic inputs 
of chlorophyll, light extinction, and irradiance all of which can be derived from remote sensing data. The LUI carbon fixation parameter was for the first time characterized for freshwater lakes from an exhaustive literature review which resulted in a straightforward model relating LUI to latitude. Prior satellite based primary productivity models are uncommon for the marine environment and exceedingly rare for freshwater systems and those that do exist are generally quite complicated with complex parametrizations making them useless outside of their regional adaptation. The simplicity of this new model can allow for estimation of phytoplankton production for anywhere in the world where only basic parameters have been measured providing an important tool for researchers moving forward marking a significant contribution to the state of the knowledge.

While this new method for estimating phytoplankton production is straightforward to implement and very useful to global studies it is not without limitations. Because the carbon fixation rate is parametrized by latitude spanning the global range (0-90 degrees) with significant natural variability, it is likely large errors in production could be encountered for any individual lake, but estimates for a broad range of lakes was found to be accurate. In light of these potential errors, this new approach should be implemented when little to know a priori information exists for any given lake system. This limitation, though, does not preclude one from developing their own parametrization of the LUI based on any number of predictive variables for their particular system of interest. However, if more complete bio-physical data exists (e.g. chlorophyll concentration, extinction coefficient, temperature, etc...) more sophisticated approaches such as the Great Lakes Production Model (GLPM) (Fee 1973, Fahnenstiel et al. 2016) or the Vertically Generalized Production Model (VGPM) (Behrenfeld and Falkowski 1997) are likely to result in more accurate production estimates. Moreover, a thrust in satellite production model development in the marine environment utilizes retrievals of phytoplankton spectral optical properties to better model wavelength resolved absorption for improved production estimates (Lohrenz et al. 2004, Lee et al. 2015). This thrust is a result, in part, of the improved capability to estimate inherent optical properties using ocean color satellite imagery. And while these approaches have shown much promise in the marine 
environment where optical properties are spatio-temporally stable and easily retrieved from space, it is still unclear whether they are applicable in optically complex freshwater systems. In these systems optical properties are difficult to estimate because of their high spatial and temporal variability which results in the violation of retrieval model assumptions. It expected with future advancements in sensor capabilities (i.e. hyperspectral, smaller spatial resolution, etc...) that these new approaches will become more common place in satellite phytoplankton production research in coastal and freshwater systems moving forward.

This dissertation work resulted in a new algorithm that utilized an approach to combine several different remote sensing products along with empirical relationships to identify extents of cyanoHABs in Lake Erie. Prior to this work, remote sensing based characterization of cyanoHABs in Lake Erie was accomplished using data from the European Space Agency (ESA) MERIS sensor and a spectral shape algorithm (e.g. Cyanobacteria Index - CI) (Wynne et al. 2008). While the results from this method have been extremely valuable to the scientific and management communities (Stumpf et al. 2012), it was not without limitations. For example, the CI did not differentiate among algal scums (i.e. harmful vs. non harmful) and sometimes produced "false positives" where blooms of non-harmful algae were classified as harmful (Millie et al. 2009). Cyanobacteria blooms are typically associated with eutrophic systems where many algae, not just cyanobacteria, bloom and the ability to differentiate specific blooms is critical. The new algorithm presented in this work, used a multi-spectral bio-optical model to estimate chlorophyll concentrations from optically complex waters. Chlorophyll values above a statistically defined threshold were related to phycocyanin, a pigment common to cyanobacteria, to identify potential areas experience HAB events. Additionally, the new method also explicitly delineated cyanobacteria surface scums which have been shown to be highly toxic (Paerl et al. 2001). Finally, the new method incorporated environmental information (e.g. water temperature) to minimize false positive identifications. Because of these algorithm improvements new insights were realized with respect to bloom formation timing and severity. 
While the new cyanoHAB mapping algorithm developed in this dissertation has provided new insights into bloom occurrences and extent, more robust approaches are needed to fully understand complex bloom dynamics in freshwater systems. The method developed here has the advantage of its inherent simplicity and can be reliably implemented for a variety of sensing platforms to generate time-series products. However, because the approach essentially identifies water with very high chlorophyll-a presence there is still a potential for false positive identifications as it is not specific for cyanoHABs. Moreover, because all phytoplankton and photosynthesizing cyanobacteria have intracellular chlorophyll-a, this method is not directly detecting spectral features characteristic of only cyanobacteria which limits quantitative assessments (Sayers et al. 2019a). Advancements made in the development of hyperspectral sensors, which possess the spectral fidelity to capture features unique to the diagnostic pigments of different algal groups, will provide the opportunity to develop approaches that provide far more detailed information with respect to cyanobacteria community structures (Wang et al. 2016, Chase et al. 2017). This type of methodology will result in far fewer false positive identifications as well as more accurate estimations of total cyanoHAB biomass or cell counts. Additionally, more advanced optical measures of the particle assemblages may be able to provide more information on the size, shape, and physiological status of individual cells or colonies which should help provide more accurate determination of specific cyanoHABs (Moore et al. 2017). Also, since cyanobacteria toxin production has been related to cellular physiological condition it may be possible to estimate toxin production from these more advanced remote sensing products.

Because cyanoHABs are highly dynamic in space and time due to rapid vertical and horizontal advection via wind driven currents, finer scale observations are desired for improved characterization. Finer spatial resolution assets are becoming more viable through the advent of cost effective imaging systems that can be deployed on aircraft and unmanned aerial systems (UAS) (Becker et al. 2019). Moreover, CubeSats are being developed which will eventually provide high resolution $(<2 \mathrm{~m})$ imagery for almost the entire globe on a daily basis (Johansen et al. 2019). With respect to finer scale temporal 
observations, geostationary platforms are being considered by the scientific community which can provide high quality images at less than 1 hour intervals (Choi et al. 2012). These observations would go a long way in furthering our understanding of how blooms form and migrate through a given freshwater system (Mouw et al. 2015).

A key result of this dissertation work is the development of new data products to fill gaps reported by the scientific community. Until this dissertation work there was no global freshwater lake chlorophyll-a data set in existence but rather there were few discrete data sets available for several lakes at different time scales that used different algorithms that were difficult to combine to draw meaningful conclusions. The new global chlorophyll data sets have been used by researchers studying a broad range of global freshwater phenomena from estimating fish production (Deines et al. 2017) to modeling flux of greenhouse gases (DelSontro et al. 2018). Also, the 20-year cyanoHAB data set produced from this work has been used to generate trends in annual HAB extent for Lake Erie and a reported directly to the EPA to evaluate their management practices to mitigate bloom occurrence. The HABs data has also been used in several studies to generate predictive models of HAB extents to help identify controlling factors and how management of these factors can reduce blooms in the future (Bertani et al. 2017, Manning et al. 2019). Additionally, the new method offered the unique ability to track the formation of extremely toxic cyanobacteria surface scum accumulations and relate those developments to environmental controlling factors. The 20-year time series analysis of surface scums revealed a dramatic increase in mean annual and maximum extent of highly toxic events over that past 10 years. Moreover, analysis of a cumulative frequency distribution "heat map" of scums revealed that scums tend to form around land masses in Lake Erie and away from the highest density of water column mixed cyanobacteria. These results suggested that the scum accumulations are formed by advection from wind driven currents more so than the assemblages mixed throughout the water column. These specific dynamics of cyanobacteria populations were previously unresolved prior to the advancements made from this dissertation work. 
For the first time spatial patterns in global freshwater phytoplankton abundance were identified. Prior to this work, much of what the community knew about global phytoplankton abundances were gleaned from literature reporting limited spatial and temporal in situ samples for individual lakes. And while this information has proved useful in global phytoplankton studies, variability in sampling protocols and measurements techniques made comparisons difficult to interpret. The remote sensing approach presented in this dissertation provided a "snapshot" in time of observable lakes and applied the same algorithm in order to facilitate comparisons of like data sets. This new capability allowed for the comparison of phytoplankton abundance at different spatial scales including by latitude and ecoregion. The latter observation provided the ability to identify regions of the world with higher and lower phytoplankton concentrations and quantify the natural variability within these regions. This analysis also identified a pathway to estimate phytoplankton abundance in lakes too small to be observed by present day satellite systems which dominant the world's freshwater by area (Verpoorter et al. 2014).

A very unique aspect of this dissertation work is the unprecedented documentation of inherent and apparent optical properties (IOP/AOP) at weekly time scales over the course of the vegetative season in Lake Erie. Previously reported IOP data sets in Lake Erie were limited in either the temporal (observations over a few days) or spatial ( $<5$ sites) domain which made it difficult to draw conclusions about how IOPs vary naturally over the course of a year or even between years (Sayers et al. 2019a). The frequent IOP/AOP sampling in the western basin of Lake Erie reported here provided unique perspectives into the transitions between dominant algal groups over seasonal cycles for two drastically different years with respect to cyanoHAB occurrence extent. For example, patterns in the mean monthly mass specific phytoplankton absorption coefficient suggested early season dominance (May-June) of large diatoms which quickly transitioned into monospecific assemblages of large cyanobacteria colonies followed by a resurgence of diatoms in the summer fall transition period. This is a critical finding for the development and application of improved remote sensing algorithms that require parameterization of optical properties such as the mass specific phytoplankton absorption coefficient. Prior to this work there was 
simply not enough data reported in the literature to capture the natural variability of these parameters which fundamentally limits the ability of remote sensing to elucidate subtle changes in phytoplankton community structures. The establishment of this very robust optical dataset allows, for the first time in the Great Lakes, the creation of the next generation of remote sensing algorithms that utilize hyperspectral radiometry to produce products far beyond the capabilities of current methods.

While this dissertation work resulted in significant advancements in the understanding of IOP/AOP variability in a cyanobacteria dominated system, improvements can be made with respect to sampling frequency and methodology moving forward. The unprecedented weekly IOP/AOP sampling reported here is an achievement only made possible through a costly dedicated federally funded monitoring program. Even with the significant resources available for this sampling activity, weekly observations are still not frequent enough to capture variability on the scale of satellite remote sensing image acquisitions (e.g. daily). This discrepancy makes accurate algorithm calibration and validation activities difficult, especially in a highly dynamic system where water column particle assemblages can significantly change sub hourly. To combat this limitation, moored instrumentation has become more common (Slade et al. 2010). Moored instruments allow for very fine temporal scale observations that can capture, for example, migration of phytoplankton transitions (Roesler and Barnard 2013) and vertical mixing of sediment particles during wind driven re-suspension events (Hill et al. 2011). These insights go a long way in helping to interpret remote sensing imagery and data products. The downside of moored optics systems is the frequent need for cleaning and calibration which again can result in significant costs for sustained implementation (Slade et al. 2010). Additionally, moored instruments are limited to a single location which drastically limits the ability to characterize IOPs throughout a spatially dynamic system.

Very recent development of optical flow through systems combat several of the limitations of discrete and moored observations (Slade et al. 2010). Flow through systems take advantage of the constant water stream of the intake cooling line of a large vessel while in 
transit. The water is redirected from the cooling line through a series of optical instruments which work in concert with a data storage device to record frequent (minutes) measurements. Because the water is continuously pumped into the ship from just below the surface, gradients in water properties can be sampled as the vessel crosses frontal boundaries. New insights have been gleaned in the marine environment where flow through systems have been implemented to survey both much of the global ocean as part of large scientific cruises funded by NASA (Boss et al. 2013). The Great Lakes are perfect for implementation of flow through optical measurements as there are multiple routine sampling efforts conducted on large ships capable of housing the needed instrumentation. These observations in conjunction with targeted discrete and moored measurements would provide for more information toward the development of new and better remote sensing models.

Current instruments to measure IOPs and AOPs have provided much information with respect to optical variability in both marine and freshwater systems. However, the present suite of standard instrumentation is unable to provide robust assessments in water types often encountered in freshwater systems. The vast majority of optical sensors have been designed with the global ocean as the primary target of observation, where particle counts are often very low relative to freshwater systems. This can pose a significant problem for some instruments such as an in situ spectrophotometer (e.g. WETLabs AC-S) that possess optical path lengths optimized for oceanic environments. These instruments can produce unreliable data when such particle counts are present that the assumption of single scattering is false, which is often the case in lake environments of high turbidity or high algal content. A new suite of sensors is needed for freshwater observations that are capable of providing valid results. Until this occurs extreme caution is needed when making measurements and processing data collected in optically complex freshwater systems.

The future of freshwater remote sensing is bright. Along with the work presented in this dissertation, others are beginning to develop bio-optical methodolologies for specific application to freshwater systems (Mishra et al. 2017). Perhaps most important at the 
fundamental level, freshwater optical properties are becoming more common place and well documented (Spyrakos et al. 2018) allowing for the advancement of algortihms capable of broad scale application in optically complex waters. Moreover, the "ocean color" community has put forth effort to identify knowledge and sensor capability gaps related to inland water remote sensing (Mouw et al. 2015, Palmer et al. 2015). Finally, the launch of operational hyperspectral sensing platforms will facilitate the next generation of water quality remote sensing products for freshwater systems. 


\section{Reference List}

Abbott, M. R., \& Letelier, R. M. 1999. Algorithm theoretical basis document chlorophyll fluorescence (MODIS product number 20). NASA

(http://www.modis.gsfc.nasa.gov/data/atbd).

Abell, R., Thieme, M.L., Revenga, C., Bryer, M., Kottelat, M., Bogutskaya, N., Coad, B., Mandrak, N., Balderas, S.C., Bussing, W. and Stiassny, M.L., 2008. Freshwater ecoregions of the world: a new map of biogeographic units for freshwater biodiversity conservation. BioScience, 58(5), pp.403-414.

Adrian, R., C. M. O’Reilly, H. Zagarese, S. B. Baines, D. O. Hessen, W. Keller, D. M. Livingstone, R. Sommaruga, D. Straile, E. Van Donk, G. A. Weyhenmeyer, and M. Winder. 2009. "Lakes as Sentinels of Climate Change." Limnology and Oceanography 54 (6, Part 2): 2283-2297. doi:10.4319/1o.2009.54.6_part_2.2283.

Alberta Lake Management Society. 2008. The Alberta Lake Management Society Volunteer Lake Monitoring Report: Amisk Lake.

Ali, K., Witter, D., \& Ortiz, J. 2014. Application of empirical and semi-analytical algorithms to MERIS data for estimating chlorophyll a in Case 2 waters of Lake Erie. Environmental Earth Sciences, 71(9), 4209-4220.

Amarasinghe, P.B. and Vijverberg, J., 2002. Primary production in a tropical reservoir in Sri Lanka. Hydrobiologia, 487(1), pp.85-93.

Babin, M., Stramski, D., Ferrari, G.M., Claustre, H., Bricaud, A., Obolensky, G. and Hoepffner, N., 2003. Variations in the light absorption coefficients of phytoplankton, nonalgal particles, and dissolved organic matter in coastal waters around Europe. Journal of Geophysical Research: Oceans, 108(C7).

Bader, H., 1970. The hyperbolic distribution of particle sizes. Journal of Geophysical Research, 75(15), pp.2822-2830.

Bailey, S.W. and Werdell, P.J., 2006. A multi-sensor approach for the on-orbit validation of ocean color satellite data products. Remote Sensing of Environment, 102(1-2), pp.1223.

Barbiero, R.P., Lesht, B.M., Hinchey, E.K. and Nettesheim, T.G., 2018. A brief history of the US EPA Great Lakes National Program Office's water quality survey. Journal of Great Lakes Research.

Bartram, J., \& Rees, G. (Eds.). 1999. Monitoring bathing waters: a practical guide to the design and implementation of assessments and monitoring programmes. CRC Press.

Barus, T. A., S. S. Sinaga, and R. Tarigan. 2008. "Phytoplankton Primary Productivity Linked to Physical-Chemical Factors Water in Water Parapat, Lake Toba.” Biological Journal of University of North Sumatra 3 (1): 11-16. 
Becker, R. H., Sultan, M. I., Boyer, G. L., Twiss, M. R., \& Konopko, E. 2009. Mapping cyanobacterial blooms in the Great Lakes using MODIS. Journal of Great Lakes Research, 35(3), 447-453.

Becker, R.H., Sayers, M., Dehm, D., Shuchman, R., Quintero, K., Bosse, K. and Sawtell, R., 2019. Unmanned aerial system based spectroradiometer for monitoring harmful algal blooms: A new paradigm in water quality monitoring. Journal of Great Lakes Research, 45(3), pp.444-453.

Behrenfeld, M. J., Westberry, T. K., Boss, E., O'Malley, R. T., Siegel, D. A., Wiggert, J. D., Franz, B. A., McClain, C. R., Feldman, G. C., Doney, S. C., Moore, J. K., Dall'Olmo, G., Milligan, A. J., Lima, I., \& Mahowald, N. 2009. Satellite-detected fluorescence reveals global physiology of ocean phytoplankton. Marine Sciences Faculty Scholarship, 111.

Behrenfeld, M., Falkowski, P., 1997a. A consumer's guide to phytoplankton primary productivity models. Limnology and Oceanography. 42, 1479-1491.

Behrenfeld, M.J. and Falkowski, P.G., 1997. Photosynthetic rates derived from satellite-based chlorophyll concentration. Limnology and oceanography, 42(1), pp.1-20.

Bennington, V., McKinley, G. A., Urban, N. R., McDonald, C.P., 2012. Can spatial heterogeneity explain the perceived imbalance in Lake Superior's carbon budget? A model study. J. Geophys. Res. 117, G03020, doi:10./029/2011JG001895.

Bergmann, T., G. Fahnenstiel, S. Lohrenz, D. Millie, and O. Schofield. 2004. The impacts of a recurrent resuspension event and variable phytoplankton community composition on remote sensing reflectance. J. Geophys. Res. 109:C10S15.

Berman, T., Stone, L., Yacobi, Y.Z., Kaplan, B., Schlichter, A., Nishri, A. and Pollingher, U., 1995. Primary production and phytoplankton in Lake Kinneret: A long-term record (1972-1993). Limnology and Oceanography, 40(6), pp.1064-1076.

Bertani, I., Primicerio, R. and Rossetti, G., 2016. Extreme climatic event triggers a lake regime shift that propagates across multiple trophic levels. Ecosystems, 19(1), pp.16-31.

Bianchi, Thomas S., Erika Engelhaupt, Per Westman, Thomas Andren, Carl Rolff, and Ragnar Elmgren. (2000) "Cyanobacterial blooms in the Baltic Sea: Natural or humaninduced?." Limnology and Oceanography 45, no. 3: 716-726.

Bierman, Victor J., Jagjit Kaur, Joseph V. DePinto, Timothy J. Feist, and David W. Dilks. (2005) "Modeling the role of zebra mussels in the proliferation of blue-green algae in Saginaw Bay, Lake Huron." Journal of Great Lakes Research 31, no. 1: 32-55.

Binding, C.E., Greenberg, T.A. and Bukata, R.P., 2012. An analysis of MODIS-derived algal and mineral turbidity in Lake Erie. Journal of Great Lakes Research, 38(1), pp.107-116. 
Binding, C.E., Greenberg, T.A., Bukata, R.P., Smith, D.E. and Twiss, M.R., 2012. The MERIS $\mathrm{MCI}$ and its potential for satellite detection of winter diatom blooms on partially icecovered Lake Erie. Journal of Plankton Research, 34(6), pp.569-573.

Binding, C.E., Greenberg, T.A., Watson, S.B., Rastin, S. and Gould, J., 2015. Long term water clarity changes in North America's Great Lakes from multi-sensor satellite observations. Limnology and Oceanography, 60(6), pp.1976-1995.

Binding, C.E., Jerome, J.H., Bukata, R.P. and Booty, W.G., 2007. Trends in water clarity of the lower Great Lakes from remotely sensed aquatic color. Journal of Great Lakes Research, 33(4), pp.828-841.

Binding, C.E., Jerome, J.H., Bukata, R.P. and Booty, W.G., 2008. Spectral absorption properties of dissolved and particulate matter in Lake Erie. Remote Sensing of Environment, 112(4), pp.1702-1711.

Bindloss, M.E., 1974. Primary productivity of phytoplankton in Loch Leven, Kinross. Proceedings of the Royal Society of Edinburgh, Section B: Biological Sciences, 74, pp.157-181.

Boss, E., Picheral, M., Leeuw, T., Chase, A., Karsenti, E., Gorsky, G., Taylor, L., Slade, W., Ras, J. and Claustre, H., 2013. The characteristics of particulate absorption, scattering and attenuation coefficients in the surface ocean; Contribution of the Tara Oceans expedition. Methods in Oceanography, 7, pp.52-62.

Boss, E., Twardowski, M.S. and Herring, S., 2001. Shape of the particulate beam attenuation spectrum and its inversion to obtain the shape of the particulate size distribution. Applied Optics, 40(27), pp.4885-4893.

Bosse, K. R., Sayers, M. J., Shuchman, R. A., Fahnenstiel, G. L., Ruberg, S. A., Fanslow, D. L., Stuart, D. G., Johengen, T. H., Burtner, A. M. 2018. Spatial-Temporal Variability of Cyanobacteria Vertical Structure in Western Lake Erie: Implications for Remote Sensing Observations. Journal of Great Lakes Research, this issue.

Bricaud, A., Babin, M., Morel, A. and Claustre, H., 1995. Variability in the chlorophyll-specific absorption coefficients of natural phytoplankton: Analysis and parameterization. Journal of Geophysical Research: Oceans, 100(C7), pp.13321-13332.

Bridgeman, T.B., Chaffin, J.D. and Filbrun, J.E., 2013. A novel method for tracking western Lake Erie Microcystis blooms, 2002-2011. Journal of Great Lakes Research, 39(1), pp.83-89.

Budd, J. W., \& Warrington, D. S. 2004. Satellite-based sediment and chlorophyll a estimates for Lake Superior. Journal of Great Lakes Research, 30, 459-466.

Budd, J. W., Beeton, A. M., Stumpf, R. P., Culver, D. A., \& Charles Kerfoot, W. 2001. Satellite observations of Microcystis blooms in western Lake Erie. Internationale Vereinigung für theoretische und angewandte Limnologie: Verhandlungen, 27(7), 3787-3793. 
Buffam, I., Turner, M. G., Desaiz, A., Hanson, P.C., Rusak, J. A., Lottig, N. R., Stanley, E. H., Carpenter, S. R. 2011. Integrating aquatic and terrestrial components to construct a complete carbon budget for a north temperate lake district. Global Change Biol. 17:1193-1211.

Buiteveld, H., Hakvoort, J.H.M. and Donze, M., 1994, October. Optical properties of pure water. In Ocean Optics XII (Vol. 2258, pp. 174-184). International Society for Optics and Photonics.

Bukata, R. P., \& Press, C. R. C. 2006. "Satellite Monitoring of Inland and Coastal Water Quality.”

Bukata, R. P., Jerome, J. H., Kondratyev, A. S., \& Pozdnyakov, D. V. 1995. Optical properties and remote sensing of coastal and inland waters. CRC Press.

Campbell, G., S. R. Phinn, A. G. Dekker, and V. E. Brando. 2011. "Remote sensing of water quality in an Australian tropical freshwater impoundment using matrix inversion and MERIS images." Remote Sensing of Environment 115(9): 2402-2414.

Campbell, I. C., C. Poole, W. Giesen, and J. Valbo-Jorgensen. 2006. "Species diversity and ecology of Tonle Sap Great Lake; Cambodia.” Aquatic Sciences 68: 355-373.

Carder, K.L., Steward, R.G., Harvey, G.R. and Ortner, P.B., 1989. Marine humic and fulvic acids: Their effects on remote sensing of ocean chlorophyll. Limnology and oceanography, 34(1), pp.68-81.

Carmichael, Wayne W. (2001) "Health effects of toxin-producing cyanobacteria:"The CyanoHABs"." Human and ecological risk assessment: An International Journal7, no. 5: 1393-1407.

Chaffin, J.D. and Bridgeman, T.B., 2014. Organic and inorganic nitrogen utilization by nitrogen-stressed cyanobacteria during bloom conditions. Journal of applied phycology, 26(1), pp.299-309.

Chase, A.P., Boss, E., Cetinić, I. and Slade, W., 2017. Estimation of phytoplankton accessory pigments from hyperspectral reflectance spectra: toward a global algorithm. Journal of Geophysical Research: Oceans, 122(12), pp.9725-9743.

Chavula, G., P. Brezonik, P. Thenkabail, T. Johnson, and M. Bauer. 2009. "Estimating chlorophyll concentration in Lake Malawi from MODIS satellite imagery." Physics and Chemistry of the Earth, Parts A/B/C 34 (13): 755-760.

Choi, J.K., Park, Y.J., Ahn, J.H., Lim, H.S., Eom, J. and Ryu, J.H., 2012. GOCI, the world's first geostationary ocean color observation satellite, for the monitoring of temporal variability in coastal water turbidity. Journal of Geophysical Research: Oceans, 117(C9). 
Chorus, Edited Ingrid, and Jamie Bartram. (1999)"Toxic cyanobacteria in water: a guide to their public health consequences, monitoring and management.".

Ciotti, A.M., Lewis, M.R. and Cullen, J.J., 2002. Assessment of the relationships between dominant cell size in natural phytoplankton communities and the spectral shape of the absorption coefficient. Limnology and Oceanography, 47(2), pp.404-417.

Codd, Geoffrey A., Louise F. Morrison, and James S. Metcalf. (2005)"Cyanobacterial toxins: risk management for health protection." Toxicology and applied pharmacology 203, no. 3: $264-272$.

Cole, J.J., Prairie, Y. T., Caraco, N. F., McDowell, W. H., Tranvik, L. J., Striegl, R. G., Duarte, C. M., Kortelainen, P., Downing, J. A., Middelburg, J. J., Melak, J. 2007. Plumbing the global carbon cycle: integrating inland waters into the terrestrial carbon budget. Ecosystems 10:171-184.

Conroy, J.D., Edwards, W.J., Pontius, R.A., Kane, D.D., Zhang, H., Shea, J.F., Richey, J.N. and Culver, D.A., 2005. Soluble nitrogen and phosphorus excretion of exotic freshwater mussels (Dreissena spp.): potential impacts for nutrient remineralisation in western Lake Erie. Freshwater Biology, 50(7), pp.1146-1162.

Cota, G. F., J. Wang, and J. C. Comiso 2004. "Transformation of global satellite chlorophyll retrievals with a regionally tuned algorithm." Remote Sensing of Environment 90 (3): 373-377.

Cullen, J.J., Ciotti, A.M., Davis, R.F. and Lewis, M.R., 1997. Optical detection and assessment of algal blooms. Limnology and Oceanography, 42(5part2), pp.1223-1239.

Darchambeau, F., Sarmento, H. and Descy, J.P., 2014. Primary production in a tropical large lake: The role of phytoplankton composition. Science of the total environment, 473, pp.178-188.

de Moraes Novo, E. M. L., C. C. de Farias Barbosa, R. M. de Freitas, Y. E. Shimabukuro, J. M., J. M. Melack, and W. Pereira Filho. 2006. "Seasonal changes in chlorophyll distributions in Amazon floodplain lakes derived from MODIS images." Limnology 7(3): 153-161.

De Stasio, Bart T., Michael B. Schrimpf, Ashley E. Beranek, and William C. Daniels. (2008) "Increased Chlorophyll a, phytoplankton abundance, and cyanobacteria occurrence following invasion of Green Bay, Lake Michigan by dreissenid mussels." Aquatic Invasions 3, no. 1:21-27.

Deines, A.M., Bunnell, D.B., Rogers, M.W., Bennion, D., Woelmer, W., Sayers, M.J., Grimm, A.G., Shuchman, R.A., Raymer, Z.B., Brooks, C.N. and Mychek-Londer, J.G., 2017. The contribution of lakes to global inland fisheries harvest. Frontiers in Ecology and the Environment, 15(6), pp.293-298. 
Dekker, Arnold Graham. (1993)."Detection of optical water quality parameters for eutrophic waters by high resolution remote sensing."

DelSontro, T., Beaulieu, J.J. and Downing, J.A., 2018. Greenhouse gas emissions from lakes and impoundments: Upscaling in the face of global change. Limnology and Oceanography Letters, 3(3), pp.64-75.

Dobson, H.F., Gilbertson, M. and Sly, P.G., 1974. A summary and comparison of nutrients and related water quality in Lakes Erie, Ontario, Huron, and Superior. Journal of the Fisheries Board of Canada, 31(5), pp.731-738.

Doerffer, R., K. Sorensen, and J. Aiken. 1999. "MERIS potential for coastal zone applications." International Journal of Remote Sensing 20: 1809-1818.

Dokulil, M., Silva, I. and Bauer, K., 1983. An assessment of the phytoplankton biomass and primary productivity of Parakrama Samudra, a shallow man-made lake in Sri Lanka. In Limnology of Parakrama Samudra—Sri Lanka (pp. 49-76). Springer, Dordrecht.

Downing, J. A., Y. T. Prairie, J. J. Cole, C. M. Duarte, L. J. Tranvik, R. G. Striegl, W. H. McDowell,P. Kortelainen, N. F. Caraco, J. M. Melack, and J. J. Middelburg. 2006. "The Global Abundance and Size Distribution of Lakes, Ponds, and Impoundments." Limnology and Oceanography 51(5): 2388-2397. doi:10.4319/lo.2006.51.5.2388.

Duan, H., Ma, R., Simis, S.G. and Zhang, Y., 2012. Validation of MERIS case-2 water products in Lake Taihu, China. GIScience \& remote sensing, 49(6), pp.873-894.

Duan, H., Ma, R., Xu, X., Kong, F., Zhang, S., Kong, W., Hao, J. and Shang, L., 2009. Twodecade reconstruction of algal blooms in China's Lake Taihu. Environmental Science \& Technology, 43(10), pp.3522-3528.

Dudgeon, D., A. H. Arthington, M. O. Gessner, Z. I. Kawabata, D. J. Knowler, C. Lévêque, R. J. Naiman, et al. 2006. "Freshwater biodiversity: importance, threats, status and conservation challenges.” Biological Reviews 81 (2): 163-182.

Dunn, O.J., 1964. Multiple comparisons using rank sums. Technometrics, 6(3), pp.241-252.

Dupouy, C., Petit, M. and Dandonneau, Y., 1988. Satellite detected cyanobacteria bloom in the southwestern tropical Pacific Implication for oceanic nitrogen fixation. REMOTE SENSING, 9(3), pp.389-396.

Edmondson, W.T., 1979. Lake Washington and the predictability of limnological events. Ergeb. Limnol., 13, pp.234-241.

Effler, S.W., Peng, F., O'Donnell, D.M. and Strait, C., 2013. The backscattering coefficient and its components in the Great Lakes: A review and synthesis. Journal of Great Lakes Research, 39, pp.108-122.

Effler, S.W., Perkins, M., Peng, F., Strait, C., Weidemann, A.D. and Auer, M.T., 2010. Lightabsorbing components in Lake Superior. Journal of Great Lakes Research, 36(4), pp.656-665. 
Engel, F., Farrell, K.J., McCullough, I.M., Scordo, F., Denfeld, B.A., Dugan, H.A., de Eyto, E., Hanson, P.C., McClure, R.P., Nõges, P. and Nõges, T., 2018. A lake classification concept for a more accurate global estimate of the dissolved inorganic carbon export from terrestrial ecosystems to inland waters. The Science of Nature, 105(3-4), p.25.

Fahnenstiel, G. L., C. Beckmann, S. E. Lohrenz, D. F. Millie, O. M. E. Schofield, and M. J. McCormick. (2002)"Standard Niskin and Van Dorn bottles inhibit phytoplankton photosynthesis in Lake Michigan." Internationale Vereinigung fur Theoretische und Angewandte Limnologie Verhandlungen 28, no. 1: 376-380.

Fahnenstiel, G. L., D. F. Millie, J. Dyble, R. W. Litaker, P. A. Tester, M. J. McCormick, R. Rediske, and D. Klarer. (2008)"Microcystin concentrations and cell quotas in Saginaw Bay, Lake Huron." Aquatic Ecosystem Health \& Management 11, no. 2: 190-195.

Fahnenstiel, G., Sayers, M. J., Shuchman, R. A., Yousef, F., Pothoven, S. A. 2016. Lake-wide phytoplankton production and abundance in the Upper Great Lakes: 2010-2013. J. Great Lakes Res. doi: 10.1016/j.jglr.2016.02.004.

Falkowski, P.G., 1981. Light-shade adaptation and assimilation numbers.Journal of plankton research, 3(2), pp.203-216.

Fee, E.J., 1973. A numerical model for determining integral primary production and its application to Lake Michigan. Journal of the Fisheries Board of Canada, 30(10), pp.1447-1468.

Fee, E.J., Shearer, J. A., DeBruyn, E. R., Schindler, E.V. 1992. Effects of lake size on phytoplankton photosynthesis. Can. J. Fish. Aquat. Sci. 49:2445-2459.

Ficke, A. D., C. A. Myrick, and L. J. Hansen. 2007. "Potential impacts of global climate change on freshwater fisheries." Reviews in Fish Biology and Fisheries 17 (4): 581-613.

Fitzwater, Steve E., George A. Knauer, and John H. Martin. (1982) "Metal contamination and its effect on primary production measurements 1." Limnology and Oceanography 27, no. 3: $544-551$.

Franz, B. 2013. "NASA Satellite Ocean Color Timeseries." Paper presented at the International Ocean Color Science Meeting, May 6-8.

Fukuda, Michael K., and Wilbert Lick. (1980) "The entrainment of cohesive sediments in freshwater." Journal of Geophysical Research: Oceans (1978-2012) 85, no. C5: 28132824.

Galat, David L., and James P. Verdin. (1989) "Patchiness, collapse and succession of a cyanobacterial bloom evaluated by synoptic sampling and remote sensing."Journal of Plankton Research 11, no. 5: 925-948.

Garno, Y. S. 2003.” Reservoir Water Quality Status, Juanda.” Journal of Technology Environment 4 (3): 128-135. 
Gelin, C., 1971. Primary production and chlorophyll a content of nanoplankton in a eutrophic lake. Oikos, pp.230-234.

Gilerson, A., Zhou, J., Hlaing, S., Ioannou, I., Schalles, J., Gross, B., Moshary, F. and Ahmed, S., 2007. Fluorescence component in the reflectance spectra from coastal waters. Dependence on water composition. Optics Express, 15(24), pp.15702-15721.

Gitelson, A. A., D. Gurlin, W. J. Moses, and T. Barrow. 2009. "A bio-optical algorithm for the remote estimation of the chlorophyll-a concentration in case 2 waters." Environmental Research Letters 4 (4): 045003. doi:10.1088/1748-9326/4/4/045003

Goldman, C.R., 1977. Trophic Status and Nutrient Loading for Lake Tahoe. North American Project: A Study of US Water Bodies, p.465.

Gons, H. J., Auer, M. T., Effler, S. W. 2008. MERIS satellite chlorophyll mapping of oligotrophic and eutrophic waters in the Laurentian Great Lakes. Remote Sensing Env. 112:4098-4106.

Gons, H. J., M. Rijkeboer, and K. G. Ruddick. 2002. “A chlorophyll-retrieval algorithm for satellite imagery (Medium Resolution Imaging Spectrometer) of inland and coastal waters.” Journal of Plankton Research 24 (9): 947-951.

Gons, H. J., M. Rijkeboer, and K. G. Ruddick. 2005. "Effect of a waveband shift on chlorophyll retrieval from MERIS imagery of inland and coastal waters.” Journal of Plankton Research 27 (1): 125-127.

Gons, H.J., 1999. Optical teledetection of chlorophyll a in turbid inland waters. Environmental Science \& Technology, 33(7), pp.1127-1132.

Gons, Herman J., Jerko H. Otten, and Machteld Rijkeboer. (1991) "The significance of wind resuspension for the predominance of filamentous cyanobacteria in a shallow, eutrophic lake." Mem. Ist. Ital. Idrobiol 48: 233-249.

Gordon, H. R., \& Brown, O. B. (1973). Irradiance reflectivity of a flat ocean as a function of its optical properties. Applied Optics, 12(7), 1549-1551.

Gower, J.F.R., Doerffer, R. and Borstad, G.A., 1999. Interpretation of the $685 \mathrm{~nm}$ peak in waterleaving radiance spectra in terms of fluorescence, absorption and scattering, and its observation by MERIS. International Journal of Remote Sensing, 20(9), pp.1771-1786.

Guildford, S.J., Bootsma, H.A., Taylor, W.D. and Hecky, R.E., 2007. High variability of phytoplankton photosynthesis in response to environmental forcing in oligotrophic Lake Malawi/Nyasa. Journal of Great Lakes Research, 33(1), pp.170-185.

Hawley, N., Johengen, T. H., Rao, Y. R., Ruberg, S. A., Beletsky, D., Ludsin, S. A., Eadie, B. J., Schwab, D. J., Croley, T. E., \& Brandt, S. B. 2006. Lake Erie hypoxia prompts Canada-US study. Eos, Transactions American Geophysical Union, 87(32), 313-319.

Hecky, R.E., Fee, E.J., Kling, H. and Rudd, J.W.M., 1978. Studies on the planktonic ecology of Lake Tanganyika. Winnipeg, Man.: Fisheries and Marine Service. 
Heim, B., H. Oberhaensli, S. Fietz, and H. Kaufmann. 2005. "Variation in Lake Baikal's phytoplankton distribution and fluvial input assessed by SeaWiFS satellite data." Global and Planetary Change 46: 9-27.

Herdendorf, C. E. 1982. Large lakes of the world. J. Great Lakes Res. 8:379-412.

Hill, P.S., Boss, E., Newgard, J.P., Law, B.A. and Milligan, T.G., 2011. Observations of the sensitivity of beam attenuation to particle size in a coastal bottom boundary layer. Journal of Geophysical Research: Oceans, 116(C2).

Ho, J. C., \& Michalak, A. M. 2015. Challenges in tracking harmful algal blooms: A synthesis of evidence from Lake Erie. Journal of Great Lakes Research, 41(2), 317-325.

Ho, J. C., \& Michalak, A. M. 2017b. Phytoplakton blooms in Lake Erie impacted by both longterm and springtime phosphorus loading. Journal of Great Lakes Research, 43(3), 221228.

Ho, J.C., Stumpf, R.P., Bridgeman, T.B. and Michalak, A.M., 2017. Using Landsat to extend the historical record of lacustrine phytoplankton blooms: A Lake Erie case study. Remote sensing of environment, 191, pp.273-285.

Holm, S., 1979. A simple sequentially rejective multiple test procedure. Scandinavian journal of statistics, pp.65-70.

Horváth, H., Kovács, A.W., Riddick, C. and Présing, M., 2013. Extraction methods for phycocyanin determination in freshwater filamentous cyanobacteria and their application in a shallow lake. European journal of phycology, 48(3), pp.278-286

$\mathrm{Hu}, \mathrm{C} ., 2009$. A novel ocean color index to detect floating algae in the global oceans. Remote Sensing of Environment, 113(10), pp.2118-2129.

Hu, C., Lee, Z., Ma, R., Yu, K., Li, D. and Shang, S., 2010. Moderate resolution imaging spectroradiometer (MODIS) observations of cyanobacteria blooms in Taihu Lake, China. Journal of Geophysical Research: Oceans, 115(C4).

Hu, C., Z. Lee, and B. Franz. 2012. "Chlorophyll a algorithms for oligotrophic oceans: A novel approach based on three-band reflectance difference." Journal of Geophysical Research 117: C01011. doi:10.1029/2011JC007395.

Huang, C., Li, Y., Yang, H., Sun, D., Yu, Z., Zhang, Z., Chen, X. and Xu, L., 2014. Detection of algal bloom and factors influencing its formation in Taihu Lake from 2000 to 2011 by MODIS. Environmental earth sciences, 71(8), pp.3705-3714.

Hunter, P. D., Tyler, A. N., Carvalho, L., Codd, G. A., Maberly, S. C. 2010. Hyperspectral remote sensing of cyanobacterial pigments as indicators for cell populations and toxins in eutrophic lake. Remote Sensing Environment 114:2705-2718. 
Ibelings, Bas W., Marijke Vonk, Hans FJ Los, Diederik T. van der Molen, and Wolf M. Mooij. (2003) "Fuzzy modeling of cyanobacterial surface waterblooms: validation with NOAA-AVHRR satellite images." Ecological Applications 13, no. 5: 1456-1472.

IOCCG (International Ocean Colour Coordinating Group). 2000. "Remote Sensing of Ocean Colour in Coastal, and Other Optically-Complex, Waters." Reports of the International Ocean-Colour Coordinating Group, edited by S. Sathyendranath, No. 3. Dartmouth, NS: IOCCG.

IOCCG (International Ocean Colour Coordinating Group). 2006. "Remote Sensing of Inherent Optical Properties: Fundamentals, Tests of Algorithms, and Applications." Reports of the International Ocean-Colour Coordinating Group. Edited by Z.-P. Lee, No. 5. Dartmouth, NS: IOCCG.

IOCCG, 2014. Phytoplankton functional types from space. Reports of the International OceanColour Coordinating Group, No. 15, p.156.

IOCCG. 2007. Ocean Colour Data Merging. In Reports of the International Ocean-Colour Coordinating Group, 5, edited by Gregg, W. W., 65. Dartmouth, Canada: IOCCG.

Jerlov, N. G. 1976. Marine optics (Vol. 14). Elsevier.

Joehnk, Klaus D., J. E. F. Huisman, Jonathan Sharples, B. E. N. Sommeijer, Petra M. Visser, and Jasper M. Stroom. (2008) "Summer heatwaves promote blooms of harmful cyanobacteria." Global change biology 14, no. 3: 495-512.

Johansen, R.A., Beck, R., Stumpf, R., Lekki, J., Tokars, R., Tolbert, C., McGhan, C., Black, T., Ma, O., Xu, M. and Liu, H., 2019. HABSat-1: Assessing the feasibility of using CubeSats for the detection of cyanobacterial harmful algal blooms in inland lakes and reservoirs. Lake and Reservoir Management, pp.1-15.

Jones, E., Oliphant, T. and Peterson, P., SciPy: Open source scientific tools for Python.[Online; accessed 2015-05-11](2001-). URL http://www. scipy. org.

Ju, J., \& Roy, D. P. 2008. The availability of cloud-free Landsat ETM+ data over the conterminous United States and globally. Remote Sensing of Environment, 112(3), 1196-1211.

Jupp, David LB, John TO Kirk, and Graham P. Harris. (1994) "Detection, identification and mapping of cyanobacteria - using remote sensing to measure the optical quality of turbid inland waters." Marine and Freshwater Research 45, no. 5: 801-828.

Kahru, M. and Elmgren, R., 2014. Multidecadal time series of satellite-detected accumulations of cyanobacteria in the Baltic Sea. Biogeosciences, 11(13), p.3619.

Kahru, M., Leppanen, J.M. and Rud, O., 1993. Cyanobacterial blooms cause heating of the sea surface. Marine Ecology Progress Series, pp.1-7. 
Kahru, M., Leppänen, J.M., Rud, O. and Savchuk, O.P., 2000. Cyanobacteria blooms in the Gulf of Finland triggered by saltwater inflow into the Baltic Sea. Marine Ecology Progress Series, 207, pp.13-18.

Kahru, M., Savchuk, O.P. and Elmgren, R., 2007. Satellite measurements of cyanobacterial bloom frequency in the Baltic Sea: interannual and spatial variability. Marine Ecology Progress Series, 343, pp.15-23.

Kalff, J. and Welch, H.E., 1974. Phytoplankton production in Char Lake, a natural polar lake, and in Meretta Lake, a polluted polar lake, Cornwallis Island, Northwest Territories. Journal of the Fisheries Board of Canada, 31(5), pp.621-636.

Kallio, K., T. Kutser, T. Hannonen, S. Koponen, J. Pulliainen, J. Vepsäläinen, and T. Pyhälahti. 2001. "Retrieval of water quality from airborne imaging spectrometry of various lake types in different seasons." Science of the Total Environment. 268 (1): 59-77.

Kane, D.D., Conroy, J.D., Richards, R.P., Baker, D.B. and Culver, D.A., 2014. Reeutrophication of Lake Erie: Correlations between tributary nutrient loads and phytoplankton biomass. Journal of Great Lakes Research, 40(3), pp.496-501.

Kanoshina, Inga, Urmas Lips, and Juha-Markku Leppänen. (2003) "The influence of weather conditions (temperature and wind) on cyanobacterial bloom development in the Gulf of Finland (Baltic Sea)." Harmful Algae 2, no. 1: 29-41.

Kauer, T., Kutser, T., Arst, H., Danckaert, T. and Nõges, T., 2015. Modelling primary production in shallow well mixed lakes based on MERIS satellite data. Remote Sensing of Environment, 163, pp.253-261.

Khondker, M., A. Alfasane, A, Gani, and S. Islam. 2012. "Limnological notes on Ramsagar, Dinajpur, Bangladesh.” Bangladesh Journal of Botany 41 (1): 119-121.

Kerekes, J., 1974. Limnological conditions in five small oligotrophic lakes in Terra Nova National Park, Newfoundland. Journal of the Fisheries Board of Canada, 31(5), pp.555583.

Kerekes, J., 1975. Phosphorus supply in undisturbed lakes in Kejimkujik National Park, Nova Scotia (Canada) With 4 figures and 2 tables in the text. Internationale Vereinigung für theoretische und angewandte Limnologie: Verhandlungen, 19(1), pp.349-357.

Klemer, A. R., Cullen, J. J., Mageau, M. T., Hanson, K. M., \& Sundell, R. A. 1996. Cyanobacterial Buoyancy Regulation: The Paradoxical Roles of Carbon. Journal of Phycology, 32(1), 47-53.

Koponen, S., K. Kallio, J. Pulliainen, J. Vepsalainen, T. Pyhalahti, and M. Hallikainen. 2004. "Water quality classification of lakes using 250-m MODIS data." Geoscience and Remote Sensing Letters, IEEE 1: 287-291. 
Korosov, A. A., D. V. Pozdnyakov, A. Folkestad, L. H. Pettersson, K. Sorensen, and R. Shuchman. 2009. "Semi-empirical algorithm for the retrieval of ecology-relevant water constituents in various aquatic environments." Algorithms 2: 470-497.

Kutser, T. 2004. Quantitative detection of chlorophyll in cyanobacterial blooms by satellite remote sensing. Limnology and Oceanography, 49(6), 2179-2189.

Kutser, Tiit. (2009) "Passive optical remote sensing of cyanobacteria and other intense phytoplankton blooms in coastal and inland waters." International Journal of Remote Sensing 30, no. 17: 4401-4425.

Lang, G.A. and Fahnenstiel, G.L., 1996. Great Lakes primary production model: methodology and use. Ann Arbor, MI: NOAA Technical Memorandum.

Larsen, S., Anderson, T., Hessen, D. O. 2011. Climate change predicted to cause severe increase in organic carbon in lakes. Global Change Biol. 17:1186-1192.

Lee, Z., (2005). A model for the diffuse attenuation coefficient of downwelling irradiance. Journal of Geophysical Research, 110(C2).http://dx.doi.org/10.1029/2004jc002275

Lee, Z., Carder, K.L. and Arnone, R.A., 2002. Deriving inherent optical properties from water color: a multiband quasi-analytical algorithm for optically deep waters. Applied optics, 41(27), pp.5755-5772.

Lee, Z., Marra, J., Perry, M.J. and Kahru, M., 2015. Estimating oceanic primary productivity from ocean color remote sensing: A strategic assessment. Journal of Marine Systems, 149, pp.50-59.

Lee, Z., Weidemann, A., Kindle, J., Arnone, R., Carder, K. L., \& Davis, C. (2007). Euphotic zone depth: Its derivation and implication to ocean-color remote sensing. Journal of Geophysical Research, 112(C3). http://dx.doi.org/10.1029/2006jc003802

Lehner, B. and Döll, P., 2004. Development and validation of a global database of lakes, reservoirs and wetlands. Journal of Hydrology, 296(1-4), pp.1-22.

Lehner, B. and P. Döll. 2004. "Development and validation of a global database of lakes, reservoirs and wetlands." Journal of Hydrology 296(1) 1-22.

Lekki, J., Deutsch, E., Sayers, M., Bosse, K., Anderson, R., \& Tokars, R., 2018. Determining Remote Sensing Spatial Resolution Requirements for the Monitoring of Harmful Algal Blooms in the Great Lakes. Journal of Great Lakes Research, this issue.

Lemoalle, J., 1981. Photosynthetic production and phytoplankton in the euphotic zone of some African and temperate lakes. Revue d'Hydrobiologie tropicale, 14(1), pp.31-37.

Lesht, B. M., R. P. Barbiero, and G. J. Warren, G. J. 2012. "Satellite ocean color algorithms: a review of applications to the Great Lakes." Journal of Great Lakes Research 38 (1): 4960 . 
Lesht, B.M., Barbiero, R.P. and Warren, G.J., 2013. A band-ratio algorithm for retrieving openlake chlorophyll values from satellite observations of the Great Lakes. Journal of Great Lakes Research, 39(1), pp.138-152.

Lewis Jr, W.M., 2011. Global primary production of lakes: 19th Baldi Memorial Lecture. Inland Waters, 1(1), pp.1-28.

Li, L., Li, L. and Song, K., 2015. Remote sensing of freshwater cyanobacteria: An extended IOP Inversion Model of Inland Waters (IIMIW) for partitioning absorption coefficient and estimating phycocyanin. Remote Sensing of Environment, 157, pp.9-23.

Li, L., Li, L., Song, K., Li, Y., Tedesco, L.P., Shi, K. and Li, Z., 2013. An inversion model for deriving inherent optical properties of inland waters: Establishment, validation and application. Remote Sensing of Environment, 135, pp.150-166.

Lohila, A., Tuovinen, J., Hatakka, J., Aurela, M., Vuorenmaa, J., Haakana, M., Laurila, T. 2015. Carbon dioxide and energy fluxes over a northern boreal lake. Boreal Env. Res. 20:474-488.

Lohrenz, S. E., G. L. Fahnenstiel, O. M.E. Schofield, and D. F. Millie. 2008. Coastal sediment dynamics and river discharge as key factors influencing coastal ecosystem productivity in southeastern Lake Michigan. Oceanography 21:54-63.

Lohrenz, S.E., Fahnenstiel, G.L., Millie, D.F., Schofield, O.M., Johengen, T. and Bergmann, T., 2004. Spring phytoplankton photosynthesis, growth, and primary production and relationships to a recurrent coastal sediment plume and river inputs in southeastern Lake Michigan. Journal of Geophysical Research: Oceans, 109(C10).

Lyche-Solheim, A., C. K. Feld, S. Birk, G. Phillips, L. Carvalho, G. Morabito, U. Mischke, et al. 2013. "Ecological status assessment of European lakes: a comparison of metrics for phytoplankton, macrophytes, benthic invertebrates and fish." Hydrobiologia 704 (1): 57-74. doi:10.1007/s10750-012-1436-y

Mann, H.B. and Whitney, D.R., 1947. On a test of whether one of two random variables is stochastically larger than the other. The annals of mathematical statistics, pp.50-60.

Manning, N.F., Wang, Y.C., Long, C.M., Bertani, I., Sayers, M.J., Bosse, K.R., Shuchman, R.A. and Scavia, D., 2019. Extending the forecast model: Predicting Western Lake Erie harmful algal blooms at multiple spatial scales. Journal of Great Lakes Research, 45(3), pp.587-595.

Maritorena, S., Siegel, D. A., \& Peterson, A. R. (2002). Optimization of a semianalytical ocean color model for global-scale applications. Appl. Opt., 41(15), 2705.http://dx.doi.org/10.1364/ao.41.002705

Marra, J., Ho, C. and Trees, C., 2003. An alternative algorithm for the calculation of primary productivity from remote sensing data. Lamont Doherty Earth Observatory Technical Report (LDEO-2003-1). 
Marzolf, G.R. and Osborne, J.A., 1972. Primary production in a Great Plains reservoir. Internationale Vereinigung für theoretische und angewandte Limnologie: Verhandlungen, 18(1), pp.126-133.

Matisoff, G., Kaltenberg, E. M., Steely, R. L., Hummel, S. K., Seo, J., Gibbons, K. J., Bridgeman, T. B., Seo, Y., Behbahani, M., James, W. F., Johnson, L. T., Doan, P., Dittrich, M., Evans, M. A., \& Chaffin, J. D. 2016. Internal loading of phosphorus in western Lake Erie. Journal of Great Lakes Research, 42(4), 775-788.

Matthews, M. W. 2011. "A current review of empirical procedures of remote sensing in inland and near-coastal transitional waters." International Journal of Remote Sensing 32 (21): 6855-6899.

Matthews, M.W. and Bernard, S., 2013. Characterizing the absorption properties for remote sensing of three small optically-diverse South African reservoirs. Remote Sensing, 5(9), pp.4370-4404.

Matthews, M.W. and Bernard, S., 2015. Eutrophication and cyanobacteria in South Africa's standing water bodies: A view from space. South African Journal of Science, 111(5-6), pp.1-8.

Matthews, M.W., 2014. Eutrophication and cyanobacterial blooms in South African inland waters: 10 years of MERIS observations. Remote Sensing of Environment, 155, pp.161177.

Matthews, M.W., Bernard, S. and Robertson, L., 2012. An algorithm for detecting trophic status (chlorophyll-a), cyanobacterial-dominance, surface scums and floating vegetation in inland and coastal waters. Remote Sensing of Environment, 124, pp.637-652.

Matthews, M.W., Bernard, S. and Winter, K., 2010. Remote sensing of cyanobacteria-dominant algal blooms and water quality parameters in Zeekoevlei, a small hypertrophic lake, using MERIS. Remote Sensing of Environment, 114(9), pp.2070-2087.

McClain, C.R., Christian, J.R., Signorini, S.R., Lewis, M.R., Asanuma, I., Turk, D. and Dupouy-Douchement, C., 2002. Satellite ocean-color observations of the tropical Pacific Ocean. Deep Sea Research Part II: Topical Studies in Oceanography, 49(13-14), pp.2533-2560.

McCormick, Michael J., and Gary L. Fahnenstiel. "Recent climatic trends in nearshore water temperatures in the St. Lawrence Great Lakes." Limnology and Oceanography 44, no. 3 (1999): 530-540.

McCree, K.J., 1981. Photosynthetically active radiation. In Physiological plant ecology I (pp. 41-55). Springer, Berlin, Heidelberg.

Megard, R.O., 1972. Phytoplankton, Photosynthesis, And Phosphorus in Lake Minnetonka, Minnesota 1. Limnology and Oceanography, 17(1), pp.68-87. 
Megard, R.O. and Smith, P.D., 1974. Mechanisms that regulate growth rates of phytoplankton in Shagawa Lake, Minnesota 1. Limnology and oceanography, 19(2), pp.279-296.

Melack, J.M., 1979. Photosynthetic rates in four tropical African fresh waters. Freshwater Biology, 9(6), pp.555-571.

Michalak, A. M., Anderson, E., Beletsky, D., Boland, S., Bosch, N. S., Bridgeman, T. B., Chaffin, J. D., Cho, K. H., Confesor, R., Daloğlu, I., DePinto, J., Evans, M. A., Fahnenstiel, G. L., He, L., Ho, J. C., Jenkins, L., Johengen, T., Kuo, K. C., Laporte, E., Liu, X., McWilliams, M., Moore, M. R., Posselt, D. J., Richards, R. P., Scavia, D., Steiner, A. L., Verhamme, E., Wright, D. M., \& Zagorski, M. A. 2013. Record-setting algal bloom in Lake Erie caused by agricultural and meteorological trends consistent with expected future conditions. Proceedings of the National Academy of Sciences, 110(16), 6448-6452.

Michalski, M.F.P., Johnson, M.G., Veal, D.M. and Brydges, T.G., 1973. Muskoka lakes water quality evaluation. Ministry of the Environment.

Millard, E.S., Myles, D.D., Johannsson, O.E. and Ralph, K.M., 1996. Phytoplankton photosynthesis at two index stations in Lake Ontario 1987-1992: assessment of the long-term response to phosphorus control. Canadian Journal of Fisheries and Aquatic Sciences, 53(5), pp.1092-1111.

Miller, P.I., Shutler, J.D., Moore, G.F. and Groom, S.B., 2006. SeaWiFS discrimination of harmful algal bloom evolution. International Journal of Remote Sensing, 27(11), pp.2287-2301.

Millie, D.F., Fahnenstiel, G.L., Bressie, J.D., Pigg, R.J., Rediske, R.R., Klarer, D.M., Tester, P.A. and Litaker, R.W., 2009. Late-summer phytoplankton in western Lake Erie (Laurentian Great Lakes): bloom distributions, toxicity, and environmental influences. Aquatic Ecology, 43(4), pp.915-934.

Mishra, D. R., Ogashawara, I., \& Gitelson, A. A. (Eds.). (2017). Bio-optical Modeling and Remote Sensing of Inland Waters. Elsevier.

Mishra, S. and Mishra, D.R., 2012. Normalized difference chlorophyll index: A novel model for remote estimation of chlorophyll-a concentration in turbid productive waters. Remote Sensing of Environment, 117, pp.394-406.

Mobley, C. D. (1994). Light and water: radiative transfer in natural waters. Academic press.

Mobley, C.D., 1999. Estimation of the remote-sensing reflectance from above-surface measurements. Applied optics, 38(36), pp.7442-7455.

Moore, T.S., Dowell, M.D., Bradt, S. and Verdu, A.R., 2014. An optical water type framework for selecting and blending retrievals from bio-optical algorithms in lakes and coastal waters. Remote sensing of environment, 143, pp.97-111. 
Moore, T.S., Mouw, C.B., Sullivan, J.M., Twardowski, M.S., Burtner, A.M., Ciochetto, A.B., McFarland, M.N., Nayak, A.R., Paladino, D., Stockley, N.D. and Johengen, T.H., 2017. Bio-optical Properties of Cyanobacteria Blooms in Western Lake Erie. Frontiers in Marine Science, 4, p.300.

Morel, A. and Prieur, L., 1977. Analysis of variations in ocean color. Limnology and oceanography, 22(4), pp.709-722.

Moses, W. J., A. A. Gitelson, S. Berdnikov and V. Povazhnyy. 2009. "Satellite Estimation of Chlorophyll-Concentration Using the Red and NIR Bands of MERIS-The Azov Sea Case Study." Geoscience and Remote Sensing Letters, IEEE 6(4): 845-849.

Moss, D., Kosten, S., Meerhoff, M., Battarbee, R. W., Jeppeson, E., Mazzeo, N., Havens, K., Lacerot, G., Liu, Z., DeMeester, L., Paerl, H, Scheffer, M. 2011. Allied attack: climate change and eutrophication. Inland Waters 1: 101-105.

Mouw, C.B., Greb, S., Aurin, D., DiGiacomo, P.M., Lee, Z., Twardowski, M., Binding, C., Hu, C., Ma, R., Moore, T. and Moses, W., 2015. Aquatic color radiometry remote sensing of coastal and inland waters: Challenges and recommendations for future satellite missions. Remote sensing of environment, 160, pp.15-30.

Mouw, C.B., Hardman-Mountford, N.J., Alvain, S., Bracher, A., Brewin, R.J., Bricaud, A., Ciotti, A.M., Devred, E., Fujiwara, A., Hirata, T. and Hirawake, T., 2017. A consumer's guide to satellite remote sensing of multiple phytoplankton groups in the global ocean. Frontiers in Marine Science, 4, p.41.

Munawar, Mohiuddin, and Iftekhar F. Munawar. (1976) "A lakewide study of phytoplankton biomass and its species composition in Lake Erie, April-December 1970." Journal of the Fisheries Board of Canada 33, no. 3: 581-600.

NASA (National Aeronautics and Space Administration). 2010. Ocean Level-2 Data Products March 22, 2010, Ocean Color Web. Accessed 11 January 2011. http://oceancolor.gsfc.nasa.gov/DOCS/Ocean_Level-2_Data_Products.pdf.

Nemirovskaya, I. A. 2012. "Variations in Different Compounds in Volga Water; Suspension; and Bottom Sediments in the Summer of 2009." Water Resources 39 (5): 533-545.

Niksic, H. 1998. GNU wget. http://www.gnu.org/software/wget/

Niu, Q., Xia, M., Rutherford, E.S., Mason, D.M., Anderson, E.J. and Schwab, D.J., 2015. Investigation of interbasin exchange and interannual variability in Lake Erie using an unstructured-grid hydrodynamic model. Journal of Geophysical Research: Oceans, 120(3), pp.2212-2232.

O’Reilly, J. E., S. Maritorena, B. G. Mitchell, D. A. Siegel, K. L. Carder, S. A. Garver, M. Kahru, and C. McClain. 1998. "Ocean Color Chlorophyll Algorithms for SEAWIFS." Journal ofGeophysical Research: Oceans 103 (C11): 24937-24953. doi:10.1029/98JC02160. 
O’Reilly, J., S. Maritorena, M. O’Brien, D. Siegel, D. Toole, D. Menzies, R. Smith, J. L. Mueller, B. G. Mitchell, M. Kahru, F. P. Chavez, P. Strutton, G. F. Cota, S. B. Hooker, C. R. McClain, K. L. Carder, F. Müller-Karger, L. Harding, A. Magnuson, D. Phinney, G. F. Moore, J. Aiken, K. R. Arrigo, R. Letelier, and M. Culver. 2000. SeaWiFS Postlaunch Calibration and Validation Analyses, Part 3. Vol 11 of NASA Technical Memorandum 2000-206892. Edited by S. B. Hooker and E. R. Firestone. Greenbelt, MD: Goddard Space Flight Center.

O’Reilly, J., S. Maritorena, M. O'Brien, D. Siegel, D. Toole, D. Menzies, R. Smith, et al. 2000. SeaWiFS Postlaunch Calibration and Validation Analyses, Part 3. Vol 11 of NASA Technical Memorandum 2000-206892 edited by S. B. Hooker and E. R. Firestone. Greenbelt, MD: Goddard Space Flight Center.

Obenour, D. R., Gronewold, A. D., Stow, C. A., \& Scavia, D. 2014. Using a Bayesian hierarchical model to improve Lake Erie cyanobacteria bloom forecasts. Water Resources Research, 50(10), 7847-7860.

Oberholster, P.J. and Botha, A.M., 2010. Use of remote sensing and molecular markers to detect toxic cyanobacterial hyperscum crust: A case study on Lake Hartbeespoort, South Africa. African Journal of Biotechnology, 9(51), pp.8791-8799.

Odermatt, D., T. Heege, J. Nieke, M. Kneubühler, and K. Itten. 2008. "Water quality monitoring for Lake Constance with a physically based algorithm for MERIS data." Sensors 8 (8): 4582-4599.

O'Donnell, D.M., Effler, S.W., Strait, C.M. and Leshkevich, G.A., 2010. Optical characterizations and pursuit of optical closure for the western basin of Lake Erie through in situ measurements. Journal of Great Lakes Research, 36(4), pp.736-746.

O'Donnell, D.M., Effler, S.W., Strait, C.M., Peng, F. and Perkins, M., 2013. Remote sensing reflectance in the Great Lakes: In situ measurements, closure analyses, and a forward model. Journal of Great Lakes Research, 39, pp.137-150.

Ondrusek, M.E., Bidigare, R.R., Waters, K. and Karl, D.M., 2001. A predictive model for estimating rates of primary production in the subtropical North Pacific Ocean. Deep Sea Research Part II: Topical Studies in Oceanography, 48(8-9), pp.1837-1863.

Otten, Timothy G., and Hans W. Paerl. (2011) "Phylogenetic inference of colony isolates comprising seasonal Microcystis blooms in Lake Taihu, China."Microbial ecology 62, no. 4: 907-918.

Ouellette, Anthony JA, Sara M. Handy, and Steven W. Wilhelm. (2006) "Toxic Microcystis is widespread in Lake Erie: PCR detection of toxin genes and molecular characterization of associated cyanobacterial communities."Microbial ecology 51, no. 2: 154-165.

Paerl, H. W., \& Ustach, J. F. 1982. Blue-green algal scums: An explanation for their occurrence during freshwater blooms. Limnology and oceanography, 27(2), 212-217. 
Paerl, H.W., Fulton, R.S., Moisander, P.H. and Dyble, J., 2001. Harmful freshwater algal blooms, with an emphasis on cyanobacteria. The Scientific World Journal, 1, pp.76-113.

Paerl, Hans W., and Joseph F. Ustach. (1982) "Blue green algal scums: An explanation for their occurrence during freshwater blooms1." Limnology and oceanography 27, no. 2: 212217.

Paerl, Hans W., Nathan S. Hall, and Elizabeth S. Calandrino. (2011) "Controlling harmful cyanobacterial blooms in a world experiencing anthropogenic and climatic-induced change." Science of the Total Environment 409, no. 10: 1739-1745.

Pahlevan, N., Z. Lee, J. Wei, C. B. Schaaf, J. R. Schott and A. Berk. 2014. “On-orbit radiometric characterization of OLI (Landsat- 8 ) for applications in aquatic remote sensing." Remote Sensing of Environment 154, 272-284.

Palmer, S.C.J., T. Kutser, and P. D. Hunter. 2015. "Remote sensing of inland waters: Challenges, progress and future directions." Remote Sensing of Environment 157: 1-8.

Patterson, G., Hecky, R.E. and Fee, E.J., 2000. Effect of hydrological cycles on planktonic primary production in Lake Malawi/Niassa. Advances in Ecological Research, 31, pp.421-430.

Peng, F. and Effler, S.W., 2010. Characterizations of individual suspended mineral particles in western Lake Erie: Implications for light scattering and water clarity. Journal of Great Lakes Research, 36(4), pp.686-698.

Peng, W.X., Wang, H.Y. and Jiang, Q.W., 2008. Dynamic change monitoring of cyanobacterial blooms using multi-temporal satel lite data in Lake Taihu. Fudan University Journal of Medical Sciences, 35(1), p.63.

Perkins, M., Effler, S.W., Peng, F., O'Donnell, D.M. and Strait, C., 2013. Light-absorbing components in the Great Lakes. Journal of Great Lakes Research, 39, pp.123-136.

Pettersson, L.H. and Pozdnyakov, D., 2012. Monitoring of harmful algal blooms. Springer Science \& Business Media.

Piccini, C., D. Conde, C. Alonso, R. Sommaruga, and J. Pernthaler. 2006. "Blooms of single bacterial species in a coastal lagoon of the southwestern Atlantic Ocean." Applied and Environmental Microbiology 72 (10): 6560-6568.

Pla, S., A. M. Paterson, J. P. Smol, B. J. Clark, and R. Ingram. 2005. "Spatial Variability in Water Quality and Surface Sediment Diatom Assemblages in a Complex Lake Basin: Lake of the Woods, Ontario, Canada." Journal of Great Lakes Research 31: 253-266.

Platt, T., 1986. Primary production of the ocean water column as a function of surface light intensity: algorithms for remote sensing. Deep Sea Research Part A. Oceanographic Research Papers, 33(2), pp.149-163. 
Pomeroy, L. R. (1974). The ocean's food web, a changing paradigm. Bioscience, 24(9), 499504.

Pope, R.M. and Fry, E.S., 1997. Absorption spectrum (380-700 nm) of pure water. II. Integrating cavity measurements. Applied optics, 36(33), pp.8710-8723.

Porta, D., Fitzpatrick, M.A. and Haffner, G.D., 2005. Annual variability of phytoplankton primary production in the western basin of Lake Erie (2002-2003). Journal of Great Lakes Research, 31, pp.63-71.

Qin, Boqiang, Guangwei Zhu, Guang Gao, Yunlin Zhang, Wei Li, Hans W. Paerl, and Wayne W. Carmichael. (2010) "A drinking water crisis in Lake Taihu, China: linkage to climatic variability and lake management." Environmental management 45, no. 1: 105 112.

Quibell, G. (1992) "Estimating chlorophyll concentrations using upwelling radiance from different freshwater algal genera." International Journal of Remote Sensing13, no. 14: 2611-2621.

Rapala, Jarkko, Kaarina Sivonen, Christina Lyra, and SEPPO I. Niemelä. (1997) "Variation of microcystins, cyanobacterial hepatotoxins, in Anabaena spp. as a function of growth stimuli." Applied and environmental microbiology 63, no. 6: 2206-2212.

Raymond, P.A., Hartmann, J., Lauerwald, R., Sobek, S., McDonald, C., Hoover, M., Butman, D., Striegl, R., Mayorga, E., Humborg, C. and Kortelainen, P., 2013. Global carbon dioxide emissions from inland waters. Nature, 503(7476), p.355.

Regnier, P., Friedlingstein, P., Ciais, P., Mackenzie, F. T., Gruber, N., Janssens, I. A., ... \& Arndt, S. (2013). Anthropogenic perturbation of the carbon fluxes from land to ocean. Nature geoscience, 6(8), 597-607.

Reinart, A. and Kutser, T., 2006. Comparison of different satellite sensors in detecting cyanobacterial bloom events in the Baltic Sea. Remote Sensing of Environment, 102(12), pp.74-85.

Revenga, C., I. Campbell, R. Abell, P. De Villiers, and M. Bryer. 2005. "Prospects for Monitoring Freshwater Ecosystems Towards the 2010 Targets." Philosophical Transactions of the Royal Society B 360 (1454): 397-413.

Reynolds, C. 2006. Ecology of Freshwater Phytoplankton. Cambridge Univ Press. Cambridge. $535 \mathrm{pp}$.

Rinta-Kanto, J. M., A. J. A. Ouellette, G. L. Boyer, M. R. Twiss, T. B. Bridgeman, and S. W. Wilhelm. (2005) "Quantification of toxic Microcystis spp. during the 2003 and 2004 blooms in western Lake Erie using quantitative real-time PCR." Environmental Science \& Technology 39, no. 11: 4198-4205.

Rinta-Kanto, Johanna M., Matthew A. Saxton, Jennifer M. DeBruyn, Juliette L. Smith, Christopher H. Marvin, Kenneth A. Krieger, Gary S. Sayler, Gregory L. Boyer, and 
Steven W. Wilhelm. (2009) "The diversity and distribution of toxigenic Microcystis spp. in present day and archived pelagic and sediment samples from Lake Erie." Harmful Algae 8, no. 3: 385-394.

Robarts, Richard D., and Tamar Zohary. (1987) "Temperature effects on photosynthetic capacity, respiration, and growth rates of bloom forming cyanobacteria." New Zealand Journal of Marine and Freshwater Research 21, no. 3: 391-399.

Roesler, C.S. and Barnard, A.H., 2013. Optical proxy for phytoplankton biomass in the absence of photophysiology: Rethinking the absorption line height. Methods in Oceanography, 7, pp.79-94.

Röttgers, R., McKee, D. and Woźniak, S.B., 2013. Evaluation of scatter corrections for ac-9 absorption measurements in coastal waters. Methods in Oceanography, 7, pp.21-39.

Rousar, D.C., 1973. Seasonal and spatial changes in primary production and nutrients in Lake Michigan. Water, Air, and Soil Pollution, 2(4), pp.497-514.

Rowe, M. D., Anderson, E. J., Wynne, T. T., Stumpf, R. P., Fanslow, D. L., Kijanka, K., Vanderploeg, H.A., Strickler, J.R., \& Davis, T. W., 2016. Vertical distribution of buoyant Microcystis blooms in a Lagrangian particle tracking model for short-term forecasts in Lake Erie. Journal of Geophysical Research: Oceans, 121(7), 5296-5314.

Saha, S., Moorthi, S., Wu, X., Wang, J., Nadiga, S., Tripp, P., Behringer, D., Hou, Y.T., Chuang, H.Y., Iredell, M. and Ek, M., 2014. The NCEP climate forecast system version 2. Journal of Climate, 27(6), pp.2185-2208.

Saulquin, B., Hamdi, A., Gohin, F., Populus, J., Mangin, A. and d'Andon, O.F., 2013. Estimation of the diffuse attenuation coefficient KdPAR using MERIS and application to seabed habitat mapping. Remote Sensing of Environment, 128, pp.224-233.

Savitzky, A. and Golay, M.J., 1964. Smoothing and differentiation of data by simplified least squares procedures. Analytical chemistry, 36(8), pp.1627-1639.

Sayers, M. J., Grimm, A. G., Shuchman, R. A., Deines, A. M., Bunnell, D. B., Raymer, Z. B., Rogers, M.W., Woelmer, W., Bennion, D. H., Brooks, C. N., Whitley, M. A.Warner, D. M., Mychek-Londer, J. 2015. A new method to generate a high resolution global distribution map of lake chlorophyll. Int. J. Remote Sensing 36:1942-1964.

Sayers, M., Fahnenstiel, G. L., Shuchman, R. A., \& Whitley, M. 2016. Cyanobacteria blooms in three eutrophic basins of the Great Lakes: a comparative analysis using satellite remote sensing. International Journal of Remote Sensing, 37(17), 4148-4171.

Sayers, M., Fahnenstiel, G.L., Shuchman, R.A. and Whitley, M., 2016. Cyanobacteria blooms in three eutrophic basins of the Great Lakes: a comparative analysis using satellite remote sensing. International Journal of Remote Sensing, 37(17), pp.4148-4171.

Sayers, M.J., Bosse, K.R., Shuchman, R.A., Ruberg, S.A., Fahnenstiel, G.L., Leshkevich, G.A., Stuart, D.G., Johengen, T.H., Burtner, A.M. and Palladino, D., 2019. Spatial and 
temporal variability of inherent and apparent optical properties in western Lake Erie: Implications for water quality remote sensing. Journal of Great Lakes Research.

Schalles, J.F. and Yacobi, Y.Z., 2000. Remote detection and seasonal patterns of phycocyanin, carotenoid and chlorophyll pigments in eutrophic waters. Ergebnisse Der Limnologie, 55, pp.153-168.

Schindler, D.W., 1972. The dependence of primary production upon physical and chemical factors in a small, senescing lake, including the effects of complete winter oxygen depletion. Arch. Hydrobiol., 69, pp.413-451.

Schindler E., K, Ashley, R. Rae, L. Vidmanic, H. Andrusak, D. Sebastian, G. Scholten et al. 2006. "Kootenay Lake Fertilization Experiment; Years 11 and 12." 2002-2003 Technical Report. Project No. 199404900; 207 electronic pages, (BPA Report DOE/BP00004029-5)

Schindler, David W., Robert E. Hecky, and Gregory K. McCullough. (2012) "The rapid eutrophication of Lake Winnipeg: Greening under global change." Journal of Great Lakes Research 38: 6-13.

Schwab, D. J., Leshkevich, G. A., \& Muhr, G. C. 1999. Automated mapping of surface water temperature in the Great Lakes. Journal of Great Lakes Research, 25(3), 468-481.

Schwab, D.J., Beletsky, D., DePinto, J. and Dolan, D.M., 2009. A hydrodynamic approach to modeling phosphorus distribution in Lake Erie. Journal of Great Lakes Research, 35(1), pp.50-60.

Schwab, David J., Brian J. Eadie, Raymond A. Assel, and Paul J. Roebber. (2006) "Climatology of large sediment resuspension events in southern Lake Michigan." Journal of Great Lakes Research 32, no. 1: 50-62.

Seekell, D. A., M. L. Pace, L. J. Tranvik, and C. Verpoorter. 2013. "A fractal-based approach to lake size-distributions.” Geophysical Research Letters 40 (3): 517-521.

Sellner, Kevin G. (1997) "Physiology, ecology, and toxic properties of marine cyanobacteria blooms." Limnology and Oceanography 42, no. 5part2: 1089-1104.

Shuchman, R., M. Sayers, C. Brooks, G. Fahnenstiel, and G. Leshkevich. 2013. A model for determining satellite- derived primary productivity estimates for Lake Michigan. J. Great Lakes Res. 39(S1):46-54.

Shuchman, Robert A., George Leshkevich, Michael J. Sayers, Thomas H. Johengen, Colin N. Brooks, and Dmitry Pozdnyakov. (2013a) "An algorithm to retrieve chlorophyll, dissolved organic carbon, and suspended minerals from Great Lakes satellite data." Journal of Great Lakes Research 39: 14-33.

Shuchman, Robert, Anton Korosov, Charles Hatt, Dmitry Pozdnyakov, Jay Means, and Guy Meadows. (2006) "Verification and application of a bio-optical algorithm for Lake 
Michigan using SeaWiFS: a 7-year inter-annual analysis."Journal of Great Lakes Research 32, no. 2: 258-279.

Simis, S.G., Ruiz-Verdú, A., Domínguez-Gómez, J.A., Peña-Martinez, R., Peters, S.W. and Gons, H.J., 2007. Influence of phytoplankton pigment composition on remote sensing of cyanobacterial biomass. Remote Sensing of Environment, 106(4), pp.414-427.

Simis, Stefan GH, Steef WM Peters, and Herman J. Gons. (2005) "Remote sensing of the cyanobacterial pigment phycocyanin in turbid inland water." Limnology and Oceanography 50, no. 1: 237-245.

Sivonen, Kaarina. (1996) "Cyanobacterial toxins and toxin production." Phycologia 35, no. 6S: $12-24$

Slade, W.H. and Boss, E., 2015. Spectral attenuation and backscattering as indicators of average particle size. Applied optics, 54(24), pp.7264-7277.

Slade, W.H., Boss, E., Dall'Olmo, G., Langner, M.R., Loftin, J., Behrenfeld, M.J., Roesler, C. and Westberry, T.K., 2010. Underway and moored methods for improving accuracy in measurement of spectral particulate absorption and attenuation. Journal of Atmospheric and Oceanic Technology, 27(10), pp.1733-1746.

Smith, D.R., King, K.W. and Williams, M.R., 2015. What is causing the harmful algal blooms in Lake Erie? Journal of Soil and Water Conservation, 70(2), pp.27A-29A.

Soares, M. C. S., M. I. de A Rocha, M. M. Marinho, S. M. F. O. Azevedo, C. W. Branco, and V. L. Huszar. 2009. "Changes in species composition during annual cyanobacterial dominance in a tropical reservoir: physical factors, nutrients and grazing effects." Aquatic Microbial Ecology 57 (2): 137.

Speziale, Barbara J., Stephen P. Schreiner, Paul A. Giammatteo, and James E. Schindler. (1984) "Comparison of N, N-dimethylformamide, dimethyl sulfoxide, and acetone for extraction of phytoplankton chlorophyll." Canadian Journal of Fisheries and Aquatic Sciences 41, no. 10: 1519-1522.

Steffen, M. M., Belisle, B. S., Watson, S. B., Boyer, G. L., \& Wilhelm, S. W. 2014. Status, causes and controls of cyanobacterial blooms in Lake Erie. Journal of Great Lakes Research, 40(2), 215-225.

Stenuite, S., Pirlot, S., HARDY, M.A., Sarmento, H., TARBE, A.L., Leporcq, B. and DESCY, J.P., 2007. Phytoplankton production and growth rate in Lake Tanganyika: evidence of a decline in primary productivity in recent decades. Freshwater Biology, 52(11), pp.2226-2239.

Strahler, A. N. 1957. "Quantitative analysis of watershed geomorphology." Eos, Transactions American Geophysical Union 38(6):913-920. 
Stramska, M., D. Stramski, R. Hapter, S. Kaczmarek, and J. Stoń. 2003. "Bio-optical relationships and ocean color algorithms for the north polar region of the Atlantic." Journal of Geophysical Research: Oceans 108 (C5). doi:10.1029/2001JC001195.

Strayer, D. L. 2010. “Alien Species in Fresh Waters: Ecological Effects, Interactions with Other Stressors, and Prospects for the Future." Freshwater Biology 55 (s1): 152-174. doi:10.1111/j.1365-2427.2009.02380.x.

Stumpf, R.P., Johnson, L.T., Wynne, T.T. and Baker, D.B., 2016. Forecasting annual cyanobacterial bloom biomass to inform management decisions in Lake Erie. Journal of Great Lakes Research, 42(6), pp.1174-1183.

Stumpf, Richard P., Timothy T. Wynne, David B. Baker, and Gary L. Fahnenstiel. (2012) "Interannual variability of cyanobacterial blooms in Lake Erie."PLoS One 7, no. 8: e42444.

Sullivan, J.M., Twardowski, M.S., Zaneveld, J.R.V., Moore, C.M., Barnard, A.H., Donaghay, P.L. and Rhoades, B., 2006. Hyperspectral temperature and salt dependencies of absorption by water and heavy water in the $400-750 \mathrm{~nm}$ spectral range. Applied Optics, 45(21), pp.5294-5309.

Tenhunen, J. D., A. Meyer, O. L. Lange, and D. M. Gates. (1980) "Development of a photosynthesis model with an emphasis on ecological applications." Oecologia45, no. 2: 147-155.

Tilahun, G. and Ahlgren, G., 2010. Seasonal variations in phytoplankton biomass and primary production in the Ethiopian Rift Valley lakes Ziway, Awassa and Chamo-The basis for fish production. Limnologica-Ecology and Management of Inland Waters, 40(4), pp.330-342.

Tranvik, L. J., Downing, J. A., Cotner, J. B., Loiselle, S. A., Striegl, R. G., Ballatore, T. J., ... \& Kortelainen, P. L. (2009). Lakes and reservoirs as regulators of carbon cycling and climate. Limnology and Oceanography, 54(6part2), 2298-2314.

Twardowski, M.S., Boss, E., Sullivan, J.M. and Donaghay, P.L., 2004. Modeling the spectral shape of absorption by chromophoric dissolved organic matter. Marine Chemistry, 89(1-4), pp.69-88.

Ulbricht, K.A., 1983. Cover photograph LANDSAT image of blue green algae in the Baltic sea. International Journal of Remote Sensing, 4(4), pp.801-802.

Vahtera, Emil, Daniel J. Conley, Bo G. Gustafsson, Harri Kuosa, Heikki Pitkänen, Oleg P. Savchuk, Timo Tamminen et al. (2007) "Internal ecosystem feedbacks enhance nitrogen-fixing cyanobacteria blooms and complicate management in the Baltic Sea." AMBIO: A journal of the Human Environment36, no. 2: 186-194.

van der Sande, C., N. van de Giesen, K. Poser, and S. Peters. 2012. EO Information Services in support of Zambezi River Basin Mapping. Water Board. Lusaka; Zambia. 
van Rijn, Jaap, and Moshe Shilo. (1985) "Carbohydrate fluctuations, gas vacuolation, and vertical migration of scum forming cyanobacteria in fishponds1." Limnology and Oceanography 30, no. 6 (1985): 1219-1228.

Vanderploeg, H.A., Liebig, J.R., Carmichael, W.W., Agy, M.A., Johengen, T.H., Fahnenstiel, G.L. and Nalepa, T.F., 2001. Zebra mussel (Dreissena polymorpha) selective filtration promoted toxic Microcystis blooms in Saginaw Bay (Lake Huron) and Lake Erie. Canadian Journal of Fisheries and Aquatic Sciences, 58(6), pp.1208-1221.

Verpoorter, C., T. Kutser, D. A. Seekell, and L. J. Tranvik. 2014. "A global inventory of lakes based on high-resolution satellite imagery." Geophysical Research Letters 41 (18): 6396-6402. doi:10.1002/2014GL060641.

Viljanen, M., V. Drabkova, V. Avinsky, L. Kapustina, and G. Raspletina. 2008. "Ecological state and monitoring of limnological and biological parameters in Lake Ladoga; Russia." Aquatic Ecosystem Health \& Management. 11 (1): 61-74.

Vincent, R. K., Qin, X., McKay, R. M. L., Miner, J., Czajkowski, K., Savino, J., \& Bridgeman, T. 2004. Phycocyanin detection from LANDSAT TM data for mapping cyanobacterial blooms in Lake Erie. Remote Sensing of Environment, 89(3), 381-392.

Vitousek, P. M., H. A. Mooney, J. Lubchenco, and J. M. Melillo. 1997. "Human Domination of Earth's Ecosystems.” Science 277: 494-499. doi:10.1126/science.277.5325.494.

Vollenweider, R.A., Munawar, M. and Stadelmann, P., 1974. A comparative review of phytoplankton and primary production in the Laurentian Great Lakes. Journal of the Fisheries Board of Canada, 31(5), pp.739-762.

Vos, R. J., J. H. M. Hakvoort, R. w. J. Jordans, and B. W. Ibelings. 2003. "Multiplatform optical monitoring of eutrophication in temporally and spatially variable lakes." Science of the Total Environment 312 (1): 221-243.

Wang, G., Lee, Z., Mishra, D.R. and Ma, R., 2016. Retrieving absorption coefficients of multiple phytoplankton pigments from hyperspectral remote sensing reflectance measured over cyanobacteria bloom waters. Limnology and Oceanography:

Methods, 14(7), pp.432-447.

Warner, D.M. and Lesht, B.M., 2015. Relative importance of phosphorus, invasive mussels and climate for patterns in chlorophyll a and primary production in Lakes Michigan and Huron. Freshwater Biology, 60(5), pp.1029-1043.

Watson, S. B., Miller, C., Arhonditsis, G., Boyer, G. L., Carmichael, W., Charlton, M. N., Confesor, R., Depew, D. C., Höök, T. O., Ludsin, S. A., Matisoff, G., MeElmurry, S. P., Murray, M. W., Richards, R. P., Rao, Y. R., Steffen, M. M., \& Wilhelm, S. W. 2016. The re-eutrophication of Lake Erie: harmful algal blooms and hypoxia. Harmful Algae, 56, 44-66. 
Wei, J., Lee, Z., \& Shang, S. (2016). A system to measure the data quality of spectral remotesensing reflectance of aquatic environments. Journal of Geophysical Research: Oceans, 121(11), 8189-8207.

Weiss, C.M. and Noore, H., 1977. The John H. Kerr Reservoir, Virginia- North Carolina. NATIONAL TECHNICAL INFORMATIQN SERVICE, p.426.

Welch, E.B., Wiederholm, T., Spyridakis, D.E. and Rock, C.A., 1977. Nutrient loading and trophic state of Lake Sammamish, Washington. North American Project--A Study of U. S. Water Bodies.

Welcomme, R. L. 2011. “An Overview of Global Catch Statistics for Inland Fish.” ICES Journal of Marine Science 68 (8): 1751-1756. doi:10.1093/icesjms/fsr035.

Werdell, P.J., McKinna, L.I., Boss, E., Ackleson, S.G., Craig, S.E., Gregg, W.W., Lee, Z., Maritorena, S., Roesler, C.S., Rousseaux, C.S. and Stramski, D., 2018. An overview of approaches and challenges for retrieving marine inherent optical properties from ocean color remote sensing. Progress in Oceanography.

Wetzel, R. G. 1983. Limnology. Saunders College Publishing. NY. 767pp.

Whalen, S.C., Chalfant, B.A., Fischer, E.N., Fortino, K.A. and Hershey, A.E., 2006. Comparative influence of resuspended glacial sediment on physicochemical characteristics and primary production in two arctic lakes. Aquatic Sciences, 68(1), pp.65-77.

Williamson, C. E., Saros, J. E, Vincent, W. F., Smol, J. P. 2009. Lakes and reservoirs as sentinels, integrators, and regulators of climate change. Limnol. Oceanogr. 54:22732282 .

Winner, R.W., 1972. An evaluation of certain indices of eutrophy and maturity in lakes. Hydrobiologia, 40(2), pp.223-245.

Wilson, M. A., and S. R. Carpenter. 1999. "Economic valuation of freshwater ecosystem services in the United States: 1971-1997.” Ecological Applications 9 (3): 772-783.

Witter, D. L., Ortiz, J. D., Palm, S., Heath, R. T., \& Budd, J. W. 2009. Assessing the application of SeaWiFS ocean color algorithms to Lake Erie. Journal of Great Lakes Research, 35(3), 361-370.

Wondie, A., S. Mengistu, J. Vijverberg, and E. Dejen. 2007. "Seasonal variation in primary production of a large high altitude tropical lake (Lake Tana; Ethiopia): effects of nutrient availability and water transparency." Aquatic Ecology 41 (2): 195-207.

Wright, J.C., 1959. Limnology of Canyon Ferry Reservoir: II. Phytoplankton Standing Crop and Primary Production. Limnology and Oceanography, 4(3), pp.235-245.

Wu, M., W. Zhang, X.Wang, and D. Luo. 2009. “Application of MODIS satellite data in monitoring water quality parameters of Chaohu Lake in China." Environmental Monitoring and Assessment 148 (1-4): 255-264. 
Wynne, T. T., Stumpf, R. P., \& Briggs, T. O., 2013. Comparing MODIS and MERIS spectral shapes for cyanobacterial bloom detection. International Journal of Remote Sensing, 34(19), 6668-6678.

Wynne, T. T., Stumpf, R. P., Tomlinson, M. C., \& Dyble, J., 2010. Characterizing a cyanobacterial bloom in western Lake Erie using satellite imagery and meteorological data. Limnology and Oceanography, 55(5), 2025-2036.

Wynne, T.T. and Stumpf, R.P., 2015. Spatial and temporal patterns in the seasonal distribution of toxic cyanobacteria in western Lake Erie from 2002-2014. Toxins, 7(5), pp.16491663.

Wynne, T.T., Stumpf, R.P., Tomlinson, M.C., Warner, R.A., Tester, P.A., Dyble, J. and Fahnenstiel, G.L., 2008. Relating spectral shape to cyanobacterial blooms in the Laurentian Great Lakes. International Journal of Remote Sensing, 29(12), pp.36653672 .

Xue, P., Schwab, D.J., Sawtell, R.W., Sayers, M.J., Shuchman, R.A. and Fahnenstiel, G.L., 2017. A particle-tracking technique for spatial and temporal interpolation of satellite images applied to Lake Superior chlorophyll measurements. Journal of Great Lakes Research, 43(3), pp.1-13.

Yacobi, Y.Z., Alberts, J.J., Takacs, M. and McElvaine, M.L., 2003. Absorption spectroscopy of colored dissolved organic carbon in Georgia (USA) rivers: the impact of molecular size distribution.

Yang, L., K. Lei, W. Meng, G. Fu, and W. Yan. 2013. "Temporal and spatial changes in nutrients and chlorophyll- $\alpha$ in a shallow lake, Lake Chaohu, China: An 11-year investigation." Journal of Environmental Sciences 25 (6): 1117-1123.

Yentsch, C. S. 1960. "The influence of phytoplankton pigments on the colour of sea water." Deep Sea Research (1953) 7 (1): 1-9.

Zhang, Y., Yin, Y., Wang, M. and Liu, X., 2012. Effect of phytoplankton community composition and cell size on absorption properties in eutrophic shallow lakes: field and experimental evidence. Optics Express, 20(11), pp.11882-11898.

Zhang, Y.L., B. Q. Qin and M. L. Liu. 2007. “Temporal-spatial variations of chlorophyll a and primary production in Meiliang Bay, Lake Taihu, China from 1995 to 2003.” Journal of Plankton Research 29 (8): 707-719.

Zohary, Tamar, and Richard D. Robarts. (1990) "Hyperscums and the population dynamics of Microcystis aeruginosa." Journal of plankton research 12, no. 2: 423-432. 